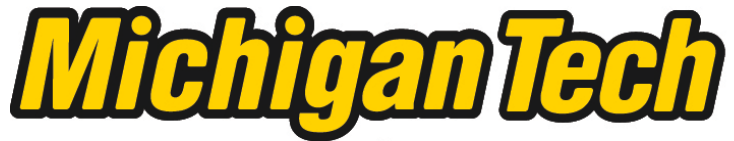 \\ Michigan Technological University Create the Future Digital Commons @ Michigan Tech
}

2015

STRUCTURES, PROPERTIES AND FUNCTIONALITIES OF

MAGNETIC DOMAIN WALLS IN THIN FILMS, NANOWIRES AND ATOMIC CHAINS: MICROMAGNETIC AND AB INITIO STUDIES

Liwei D. Geng

Michigan Technological University

Follow this and additional works at: https://digitalcommons.mtu.edu/etds

Part of the Materials Science and Engineering Commons, Nanoscience and Nanotechnology Commons, and the Physics Commons

Copyright 2015 Liwei D. Geng

\section{Recommended Citation}

Geng, Liwei D., "STRUCTURES, PROPERTIES AND FUNCTIONALITIES OF MAGNETIC DOMAIN WALLS IN THIN FILMS, NANOWIRES AND ATOMIC CHAINS: MICROMAGNETIC AND AB INITIO STUDIES", Dissertation, Michigan Technological University, 2015.

https://doi.org/10.37099/mtu.dc.etds/1008

Follow this and additional works at: https://digitalcommons.mtu.edu/etds

Part of the Materials Science and Engineering Commons, Nanoscience and Nanotechnology Commons, and the Physics Commons 


\title{
STRUCTURES, PROPERTIES AND FUNCTIONALITIES \\ OF MAGNETIC DOMAIN WALLS IN THIN FILMS, NANOWIRES AND ATOMIC CHAINS: \\ MICROMAGNETIC AND AB INITIO STUDIES
}

\author{
By \\ Liwei D. Geng \\ A DISSERTATION \\ Submitted in partial fulfillment of the requirements for the degree of \\ DOCTOR OF PHILOSOPHY \\ In Materials Science and Engineering \\ MICHIGAN TECHNOLOGICAL UNIVERSITY \\ 2015 \\ (C) 2015 Liwei D. Geng
}


This dissertation has been approved in partial fulfillment of the requirements for the Degree of DOCTOR OF PHILOSOPHY in Materials Science and Engineering.

Department of Materials Science and Engineering

Dissertation Advisor: Yongmei M. Jin

Committee Member: $\quad$ Stephen L. Kampe

Committee Member: $\quad$ Stephen A. Hackney

Committee Member: $\quad$ Keat Ghee Ong

Department Chair: Stephen L. Kampe 
Tomy wife 


\section{Table of Contents}

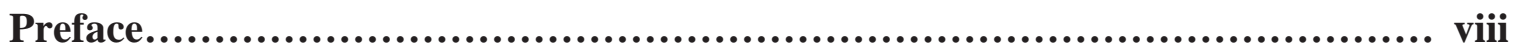

Acknowledgement............................................................... ix

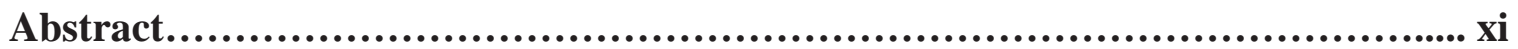

Chapter 1. Introduction............................................................... 1

1.1 Magnetic Domain-Wall-based Devices..................................... 1

1.2 Current Induced Domain Wall Motion....................................... 3

1.2.1 Spin Transfer Torques.............................................. 3

1.2.2 Spin-Orbit Torques.................................................. 5

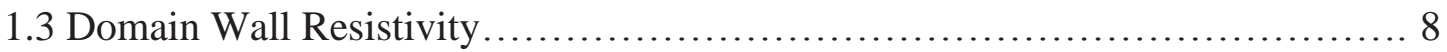

1.4 Magnetic Domain Walls in Thin Films, Nanowires and Atomic Chains.......... 11

Chapter 2. Computational Methods.....................................................14

2.1 Micromagnetic Modeling................................................... 14

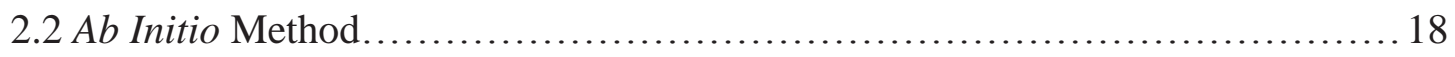

Chapter 3. Magnetic Domain Walls in Thin Films..................................22

3.1 Domain Wall Creep in an Ultrathin Magnetic Film with a Driving

Field near the Depinning Force........................................... 22

3.1.1 Introduction..................................................... 22

3.1.2 Micromagnetic Model.............................................. 23

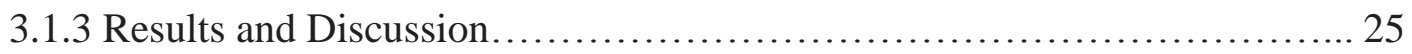

3.1.4 Conclusion..................................................... 32

3.2 Roughness, Creep, Depinning and Flow of Domain Wall Line

with Substructures in an Ultrathin Magnetic Film............................. 34

3.2.1 Introduction............................................................... 34

3.2.2 Micromagnetic Model................................................ 36

3.2.3 Statics of Domain Wall Lines with Substructures .......................... 38

3.2.4 Dynamics of Domain Wall Lines with Substructures...................... 48

3.2.4.1 Flow Regime................................................. 48

3.2.4.2 Depinning Regime........................................... 51

3.2.4.3 Creep Regime................................................ 54 
3.2.5 Conclusion.................................................... 58

3.3 Current Induced Bloch Line Motion and Ultrafast Core Reversal............... 61

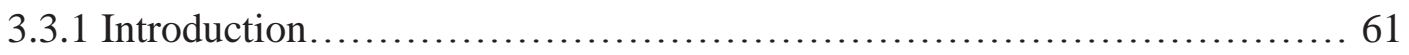

3.3.2 Current Induced Bloch Line Motion in Bulk......................... 63

3.3.3 Current Induced Bloch Line Motion in Thin Film........................ 71

3.3.4 Theoretical Analysis............................................ 74

3.3.5 Ultrafast Reversal of Bloch lines in Thin Film......................... 81

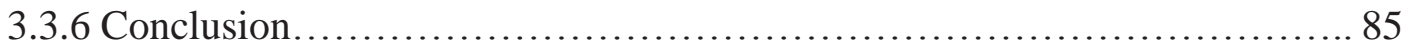

3.4 Future Research.................................................. 87

Chapter 4. Magnetic Domain Walls in Nanowires.................................. 89

4.1 Nanometer Magnetic Transverse Domain Wall Logic.......................... 89

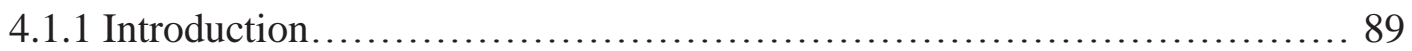

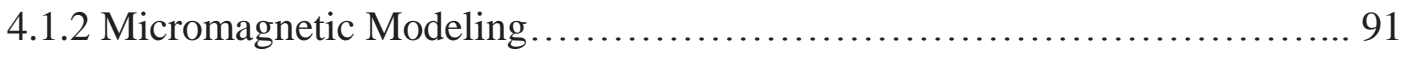

4.1.3 Results and Discussion.......................................... 93

4.1.3.1 Data Generator.............................................. 93

4.1.3.2 NOT ..................................................... 98

4.1.3.3 AND/OR................................................... 101

4.1.4 Conclusion..................................................... 108

4.2 Generator and Shift Register Based on $360^{\circ}$ Domain Walls.................. 110

4.2 .1 Introduction.................................................. 110

4.2.2 $360^{\circ}$ Domain Wall Generator......................................... 111

4.2.3 $360^{\circ}$ Domain Wall Shift Register.................................... 118

4.2.4 Conclusion................................................... 122

4.3 Altering Critical Depinning Current via Domain Wall Pile-up in Magnetic

Nanowires...................................................... 123

4.3.1 Introduction................................................... 123

4.3.2 Micromagnetic Modeling ....................................... 125

4.3.3 Results and Discussion......................................... 127

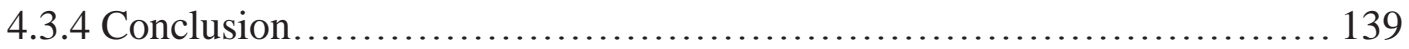

4.4 Current Induced Domain Wall Depinning and Transportation through 
Planar Nanowire Circular Geometry by Spin Transfer Torques

and Spin-orbit Torques................................................ 140

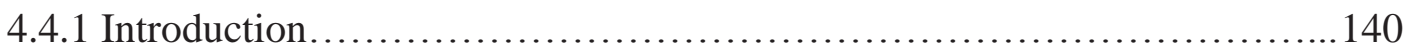

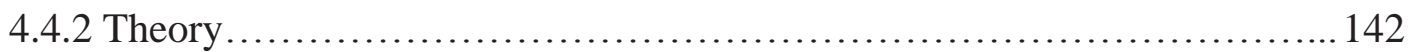

4.4.3 Results and Discussion............................................ 144

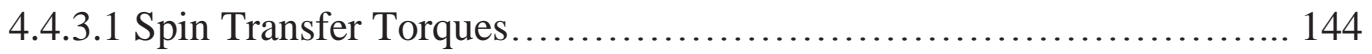

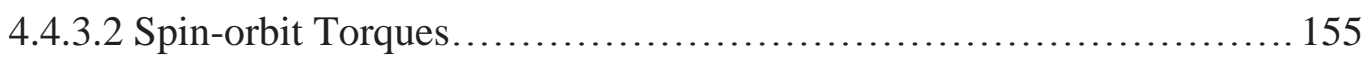

4.4.3.2.1 Rashba Effect................................................ 155

4.4.3.2.2 Spin-Hall Effect........................................ 162

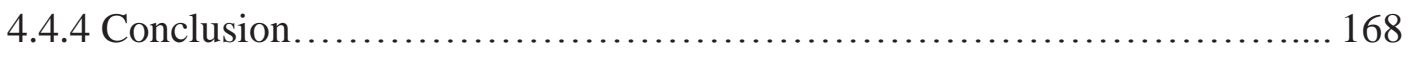

4.5 Magnetic Vortex Racetrack Memory ....................................... 170

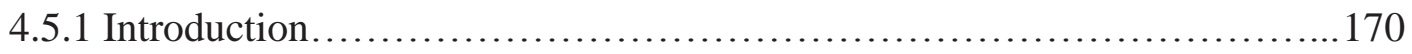

4.5.2 Micromagnetic Modeling .......................................... 171

4.5.3 Densely Packed Magnetic Vortices...................................... 173

4.5.4 Magnetic Vortex Racetrack Memory................................... 177

4.5.5 Conclusion....................................................... 184

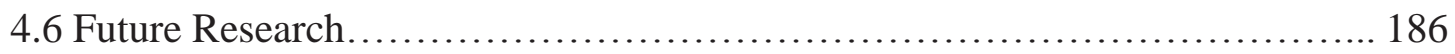

Chapter 5. Magnetic Domain Walls in Atomic Chains............................... 187

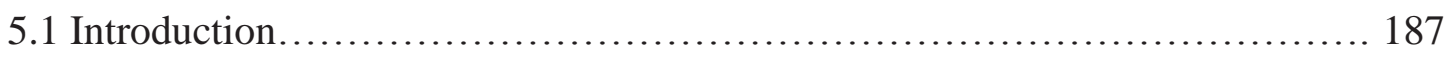

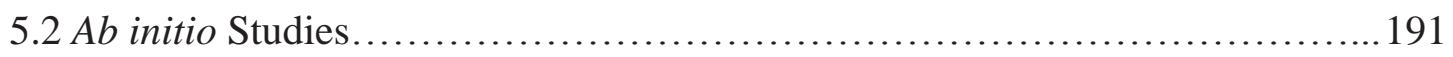

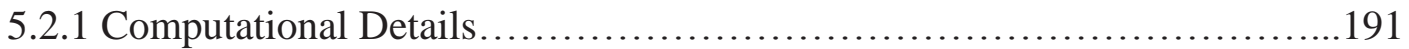

5.2.2 Magnetic Parameters at Zero Temperature............................... 192

5.2.3 Magnetic Parameters at Finite Temperature.............................. 203

5.3 Spin Dynamics Simulations............................................. 208

5.3.1 Atomistic Model........................................................ 208

5.3.2 Magnetic Domain Wall.............................................. 211

5.3.3 Magnetic Domain Stability and Switching..............................2216

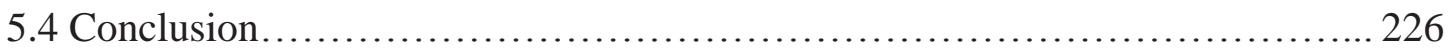

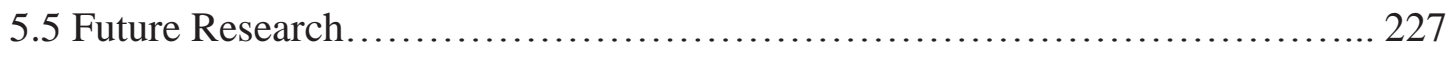

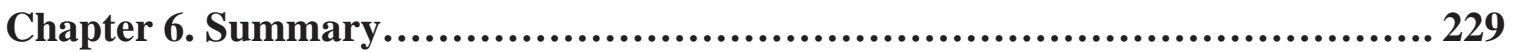


6.1 Magnetic Domain Walls in Thin Films.................................. 229

6.2 Magnetic Domain Walls in Nanowires.................................. 231

6.3 Magnetic Domain Walls in Atomic Chains................................ 234

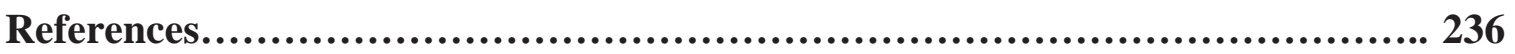

Appendix A: Domain Wall with Substructures in Ideal Magnetic Film.............. 251

Appendix B: Domain Wall with Substructures in Defective Magnetic Film......... 255

Appendix C: Copyright Permission of Section 4.2 and Section 6.2................ 257

Appendix D: Copyright Permission of Section 4.3 and Section $6.2 \ldots \ldots \ldots \ldots \ldots \ldots . \ldots . \ldots 259$ 


\section{Preface}

Magnetic domain walls play very important role in magnetics, spintronics, and magnonics, and lots of domain-wall-based devices have been proposed and constructed in recent years. Structures, properties and functionalities of magnetic domain walls in thin film, nanowires and atomic chains are computationally studied in this dissertation.

All the researches described in this dissertation were conducted under the supervision of Prof. Yongmei M. Jin in the Department of Materials Science and Engineering, Michigan Technological University, between September 2010 and August 2015. In this dissertation, part of work has been published in 2 peer-reviewed papers: Section 4.2 is reprinted from the paper [Geng, Liwei D. and Jin, Yongmei M., Generation and storage of $360^{\circ}$ domain walls in planar magnetic nanowires. Journal of Applied Physics, 2012. 112(8): p. 083903], and Section 4.3 is reprinted from the paper [Geng, Liwei D. and Jin, Yongmei M., Altering critical depinning current via domain wall pileup in magnetic nanowires. Journal of Magnetism and Magnetic Materials, 2015. 393(0): p. 121-126]. For those papers, I performed the simulation work, collected and analyzed the data, and prepared the draft, while Prof. Jin developed the simulation model, programmed the code, analyzed the simulation results, and revised the draft very carefully. The remained work is unpublished, but will be submitted for publication in the near future. 


\section{Acknowledgement}

First and foremost I would like to express my deepest gratitude to my advisor, Prof. Yongmei M. Jin, for her invaluable guidance, continuous encouragement and everlasting support during my Ph.D. pursuit in past five years. She taught me the essential knowledge of fundamental mathematical calculations and programing techniques in the field of computational materials simulation. She often encouraged me to explore, and gave me much freedom to do the topics I am interested in. Her insightful suggestions really helped me much in my research work, and I really learned much on how to do good research. I gratefully appreciate all her contributions of time, ideas, and funding to my Ph.D. research work. She is always very nice, and her kindness made the communication between us very harmonious. She also concerns my life very much, which always made me feel very warm. I am so fortunate to have such a great advisor, without her, this dissertation will not be possible.

I would also gratefully and deeply thank Prof. Yu U. Wang for his professional guidance in ferroelectric materials and essential teachings in fundamental physical calculations and simulation modeling in computational ferroelectrics during my Ph.D. pursuit. Every discussion between us is always helpful and valuable, which made my relevant research of much efficiency. He is very rigorous and enthusiastic in doing research, and always have many interesting and valuable ideas, which impressed me very much and sets the norm for me in my future career. I also gratefully appreciate all his contributions of time and ideas to my Ph.D. research work. 
I would like to thank my committee members, Prof. Stephen L. Kampe, Prof. Stephen A. Hackney and Prof. Keat Ghee Ong, for their helpful advice and kindness to review my dissertation. I would also thank Prof. Douglas J. Swenson, , Prof. Peter D. Moran, Prof. Stephen A. Hackney, Prof. Mark R. Plichta, and Prof. Miguel Levy for their help and teachings of my graduate courses. Thanks to Edward A. Laitila, Owen P Mills, Ruth I. Kramer, Paul Fraley, Margaret Rothenberger, and Beth Sickler, for their persistent help and support in the past few years.

I am grateful to my research group members and friends, Dr. Tianle Cheng, Dr. Igor Kuianov, Jie Zhou, Fengde Ma, Zachary Morgan, Weilue He, Fei Li, Hui Wang, Yu and others, for their assistance and support during the past five years in Houghton.

Finally, I would like to express my deep gratitude to my parents for their selfless support, understanding and solicitude, as well as to my younger sister. Special thanks to my wife, Ran Ding, for her persistent support, constant companion, and meticulous care in those many years. Final special thanks to all of my spirit teachers, who always give me much strength to carry on. 


\begin{abstract}
Structures, properties and functionalities of magnetic domain walls in thin film, nanowires and atomic chains are studied by micromagnetic simulations and $a b$ initio calculations in this dissertation. For magnetic domain walls in thin films, we computationally investigated the dynamics of one-dimensional domain wall line in ultrathin ferromagnetic film, and the exponent $\alpha=1.24 \pm 0.05$ is obtained in the creep regime near depinning force, indicating the washboard potential model is supported by our simulations. Furthermore, the roughness, creep, depinning and flow of domain wall line with commonly existed substructures driven by magnetic field are also studied. Our simulation results demonstrate that substructures will decrease the roughness exponent $\zeta$, increase the critical depinning force, and reduce the effective creep energy barrier. Current induced domain-wall substructure motion is also studied, which is found quite different from current induced domain wall motion.
\end{abstract}

For magnetic domain walls in nanowires, field and current induced domain wall motion is studied, and some relevant spintronic devices are proposed based on micromagnetic simulations. Novel nanometer transverse-domain-wall-based logic elements, $360^{\circ}$ domain wall generator and shift register are proposed. When spinpolarized current is applied, the critical current for domain wall depinning can be substantially reduced and conveniently tuned by controlling domain wall number in the pile-up at pinning site, in analogy to dislocation pile-up responsible for Hall-Petch effect in mechanical strength. Furthermore, threshold currents for domain wall depinning and transportation through circular geometry in planar nanowire induced by spin transfer 
torques and spin-orbit torques are theoretically calculated. In addition, magnetic vortex racetrack memory which combines both conceptions of magnetic vortex domain walls and racetrack is also proposed using micromagnetic simulations.

For magnetic domain walls in $\mathrm{Ni}$ atomic chains, a truly magnetic domain wall structure and the single domain switching process are investigated by both ab initio studies and spin dynamics simulations. Spin moment softening effect caused by the hybridization effect between two spin channels is considered. The atomic domain wall as narrow as 4 atom-distance with slight spin moment softening effect indicates a relatively evident ballistic magnetoresistance effect, and the large $E_{B}$ indicates the strong stability of single domain state. 


\section{Chapter 1. Introduction}

\subsection{Magnetic Domain-Wall-based Devices}

Magnetic materials have been widely used as functional devices in a long history, such as recording devices including hard disk drives (HDDs), magnetoresistive randomaccess memory (MRAM), bubble memory and thin-film memory, sensor devices, domain shift register devices and so on. [1] Most of those traditional functional devices are based on magnetic domains, for example, almost all the recording media use single domains with two opposite polarities or magnetizations to represent information bit 0 and 1 . Magnetic domain walls which separate neighboring domains always influence some specific domain behaviors like domain nucleating, switching or stabilizing, and hence to directly or indirectly affect the relevant functionalities performed by magnetic domains. For example, the existence of domain walls for thin-film memory and bubble memory can affect the domain state as well as the domain propagation to somewhat large extent. [1] Therefore, besides of domain itself, magnetic domain wall is also very crucial. Unfortunately, the size of domain wall is usually much smaller compared to the domain, which traditionally made researches on domain walls, especially narrow domain walls, very difficult. However, the development of nanotechnology makes investigations on magnetic domain walls possible and much attention has been paid to the small size domain walls. Up to present, much progress on domain walls has been done and many unique properties accompany with the corresponding functionalities have also been

revealed, which results in novel spintronic devices functioned by magnetic domain walls with miniaturized size, called domain-wall-based devices, for which magnetic domain 
walls play very important role. [2-35] Briefly speaking, almost all of those novel functionalities in magnetics and spintronics are related to the static structure and dynamic behavior of domain walls, and the two factors will be briefly introduced in the following.

As for the structure, since several different structure types can be occupied by magnetic domain walls and each type also occupies distinguishable spin textures, domain wall itself can also be treated as information carriers which might be distinguished by either domain wall chirality [36-39] or vortex core polarity [20, 40-45], or even by the present or absence of a domain wall [19, 21, 46, 47]. For instance, a $180^{\circ}$ head-head or tail-tail transverse domain wall in a planar Permalloy nanowire can either point 'upward' or 'downward' determined by the magnetization rotation chirality, either counterclockwise or clockwise, while a vortex or antivortex domain wall in a relatively wider nanowire or a circular disk can also be distinguished by core polarity besides of the chirality. Such a flipping or switching between two states of a domain wall spin texture can be realized by applying an external stimuli such as magnetic field [40-42], spinpolarized current $[20,36,44]$, heat or laser pulses [48] and so on, indicating potential applications in domain-wall based recording media.

Under proper external driving force, domain walls can be moved, and many appealing magnetic and spintronic devices have been proposed due to the movable domain walls. When both nanowire circuits with simple geometric design and rotation magnetic field which also acts as a clock signal simultaneously are applied, a complete logic architecture can be constructed, which is called magnetic domain-wall logic, with the advantages of great simplicity, low power consumption and potentiality to build 3D networks, promising an alternate route to traditional semiconductor electronics. [32, 34] 
In particular, since the observation of spin-polarized current induced domain wall motion $[3,5,6,18]$ which will be introduced in detail in Sec. 1.2, many attracting novel operations have been proposed, such as current-controlled magnetic domain-wall nanowire shift register [19] and magnetic domain-wall racetrack memory [21]. The latter proposes a high density memory which can be realized by shifting domain walls stored in nanowries with in-plane magnetocrystalline anisotropy by applying current pulses, which is further developed by using nanowires with perpendicular magnetocrystalline anisotropy in order to reduce the required spin-polarized current density [46].

In addition to the above potential applications based on domain walls in magnetics and spintronics, domain wall is also of great significance in the field of magnonics. For example, domain wall can induce a phase shift in propagation spin waves, which promises a domain-wall based magnonic logic device performing as NOT or XOR Boolean operator. $[49,50]$ Besides, the ultrafast reversal of a domain wall or vortex core can induce strong spin wave radiation $[20,41]$, which will result in a domainwall based spin wave generator. Thus, due to the important role of domain wall in magnetics, spintronics and magnonics, the properties as well as the relevant functionalities of magnetic domain walls are focused in this dissertation.

\subsection{Current Induced Domain Wall Motion}

\subsubsection{Spin Transfer Torques}

Generally speaking, magnetic domain walls can be controlled in four ways, magnetic field control, spin-polarized current control, electric field control and photonic field control. [23] In this dissertation, domain wall properties and corresponding 
functionalities controlled by the former two ways are focused. Since the conventional magnetic-field-induced domain wall dynamics has been almost well understood in last century while the study on current-induced domain wall motion is relatively new, the domain wall motion behavior induced by intrinsic spin transfer torques and extrinsic interface-derived spin-orbit torques will be briefly introduced in Sec. 1.2.1 and 1.2.2, respectively.

Conduction electrons carry not only charges, but also spins. Unlike normal metals like $\mathrm{Cu}$ or $\mathrm{Au}$ in which conduction electrons are not polarized when injected in, transporting conduction electrons in ferromagnetic transition-metals like Ni or Co will be spin-polarized due to the asymmetric majority and minority channels for d electrons. When the spin-polarized current passes across the domain wall, all the incident electrons' spins will be flipped, i.e., a spin-up electron ends up with a spin-down state after crossing an $180^{\circ}$ domain wall, which exerts a torque on the magnetizations within domain wall due to the angular momentum conservation effect, and such a torque is called adiabatic torque $[3,18,51]$. When the adiabatic torque is exerted on a domain wall, magnetizations with the domain wall will rotate due to the effect of Larmor precession, and hence such a torque is only able to tilt or flip a domain wall without really driving it to move steadily. Besides of adiabatic torque, the other kind of torque induced by spin-polarized current, nonadiabatic torque $[8,18,51]$, also exists. The nonadiabatic torque is treated as corrections to ideal adiabaticity and pure local spin transfer and have been claimed the key mechanism to cause the steady motion of domain wall. Both the adiabatic and nonadiabatic torques can be expressed phenomenologically by inserted as two additional terms into the conventional Landau-Lifshitz-Gilbert (LLG) equation $[3,8,51]$ (see details 
in Sec. 2.1). Unlike the adiabatic term, the origin of the nonadiabatic term (also called $\beta$ term) is more controversial. Zhang and Li proposed that the nonadiabatic term is due to the spin-flip scattering [8], while Stiles etc. cast doubt on that statement by reporting the quantum and semiclassical calculations without treating scattering [52]. Although there is so much debate on the $\beta$-term, a great deal of experimental $[4,6,15,16,28,29]$ and theoretical $[3,5,52-56]$ investigations accompany with the computational studies $[51,57-$ 60] based on the modified LLG equation have been done and then the reasonability of that term is demonstrated.

Actually, the above two spin transfer torques as well as the modified LLG equation is applied only to magnetic systems with relatively wide domain walls. When the domain wall becomes extremely narrow, momentum transfer [5] instead of spin transfer effect will be dominant to the current induced domain wall motion. Unfortunately, since the momentum-transfer torque is nonlocal unlike the spin transfer torques, it is difficult to be phenomenologically added as an individual term to the LLG equation, but in a certain approximation, such term can also be expressed in the same form with the $\beta$-term and then only the nonadiabatic parameter $\beta$ is required to be modified if momentum-transfer torque is also taken into account [61]. In this dissertation, all the domain walls we computationally studied are relatively wide, so that the momentum-transfer effect is ignored.

\subsubsection{Spin-Orbit Torques}

The conventional spin-transfer torques including both the adiabatic torque and the non-adiabatic torque are intrinsic and able to drive the DW moving in the direction of 
conduction electrons. Unlike those conventional torques, there are also some other torques which are not directly due to the coupling of spin-polarized current and local magnetizations, which are usually called extrinsic torques. Among those extrinsic torques, spin-orbit torques which are derived from the interface between ferromagnetic layer and top/bottom layer have attracted much interest in recent years. Usually, there are two main effects, Rashba effect [62] and Spin-Hall effect [63], contributed to the spinorbit torques. Both effects require a nonmagnetic heavy metal layer with strong spin-orbit interactions. Since spin-orbit torques are interface-derived, one can expect that a thin ferromagnetic layer should be required to enhance the interface effect or spin-orbit torques effect.

Rashba effect is usually arisen from a structure inversion asymmetry of two different interfaces, heavy-metal/ferromagnetic and ferromagnetic/oxide interface, and a typical system is the heavy-metal/ferromagnetic/oxide heterostructure like $\mathrm{Pt} / \mathrm{Co} / \mathrm{AlO}$ stack $[37,38]$. The induction magnetic field caused by the spin-orbit interaction is given by $\mathbf{B}_{S O}=-(\mathbf{v} \times \mathbf{E}) /\left(2 c^{2}\right)$, where $\mathbf{E}=-\nabla V$ is the electric field originating from the asymmetric crystal field $V$ and $\mathbf{v}$ is the electron's velocity [64]. Since the interfacial electric field is usually perpendicular to the surface and electrons move within the surface plane, the effective field induced by Rashba effect will be $\mathbf{H}_{R} \propto \hat{\mathbf{z}} \times \mathbf{j}$ which is perpendicular to both the surface and injected current direction. [37, 38, 65, 66] Since Rashba effect can give rise to an effective transverse field, the Rashba torque is also called field-like torque $[67,68]$. Therefore, one can expect that Rashbe effect is able to either stabilize or flip a domain wall depending on the incident current direction, domain 
wall chirality and the heterostructure layer arrangement, which have also been revealed by recent investigations [37, 38, 65-67, 69].

For spin Hall effect, a typical system exhibiting strong spin-orbit torque effect usually includes the heavy-metal/ferromagnetic interface, and hence heterostructure stacks such as $\mathrm{Pt} / \mathrm{Co} / \mathrm{Pt}, \mathrm{Pt} / \mathrm{CoFe} / \mathrm{MgO}, \mathrm{Ta} / \mathrm{CoFe} / \mathrm{MgO}$, and $\mathrm{Ta} / \mathrm{CoFeB} / \mathrm{MgO}$ are often experimentally adopted [70-72]. The strong spin-orbit effect makes spin-up and spindown electrons in the heavy-metal layer deflect in opposite directions toward the interface to form a transverse spin current which will be injected into the ferromagnetic layer to exert a torque. The spin-Hall torque is also called Slonczewski torque, which can act as an anti-damping torque and even able to make the DW move along the current direction, in contrary to the conventional spin transfer torque effect. [67, 68, 70-75] Precisely speaking, whether the domain wall moves in the electron direction or current direction depends on both domain wall chirality and spin-Hall angle. The spin-Hall angle denoted as the ratio of the spin current to the charge current densities can be either positive or negative depending on the substrate material [70, 71]. Extensive investigations on this interesting and unique effect have been done with a great deal of attractive potential applications in spintronics being proposed. [39, 67-76]

One can expect that both the Rashba effect and spin Hall effect could significantly influence the domain wall dynamic properties. Like the conventional spin transfer torques, the spin-orbit torques excited by those two effects can be also phenomenologically added to the modified LLG equation, which will be discussed in Sec. 2.1. Actually, in addition to the above two effects, the Dzyaloshinskii-Moriya interaction (DMI) [77-80] which is also owing to the spin-orbit interaction effect plays an 
important role in magnetic domain wall properties. Also derived from the interface, DMI arises from an interfacial anisotropic exchange effect which can be described by the DMI energy $E_{D M I}=\mathbf{D}_{i j} \cdot\left(\mathbf{S}_{i} \times \mathbf{S}_{j}\right)$, where $\mathbf{D}_{i j}$ is the Dzyaloshinskii vector. DMI makes the domain walls with different chiralities occupy different energy levels, so that domain wall structure with only one certain chirality is energetically preferred. In another word, DMI can stabilize the domain wall chirality, and that is why the domain wall in $\mathrm{Pt} / \mathrm{Co} / \mathrm{Pt}$ stack always moves in the current direction under the spin Hall effect. [39, 71, 75]

\subsection{Domain Wall Resistivity}

In analogy to the giant magnetoresistance (GMR) [81] which arises from the spindependent scattering by the antiparallel adjacent ferromagnetic layers, magnetic domain wall separating adjacent antiparallel domains may also contribute a resistance which is usually called domain wall magneto-resistance (DWMR). In 1996, the first clear indication of giant GMR effects in a homogeneous magnetic system due to domain wall scattering was reported by Gregg et al. [82] After that, Levy and Zhang theoretically calculated the DWMR using the same Hamiltonian as used to explain GMR, and it is indicated that the amount of MR that is attributable to domain wall scattering for the current perpendicular to wall (CPW) configuration is between $2 \%$ and $11 \%$, consistent with the 5\% found by Gregg. [81] In contrary to the positive DWMR mentioned above, negative value was also reported [83], for which Tatara and Fukuyama also calculated the domain wall resistivity based on the linear response theory to show that the wall contributes to the decoherence of electrons and it is this quantum correction that leads to 
a decrease of resistivity [84]. Although lots of experimental studies [85-89] are made to measure the DWMR hereafter, both positive and negative values were still reported, revealing the difficulty to determine the sign as well as the exact physical origin of DWMR. Gorkom et al. attribute the DWMR to the spin-dependent scattering lifetimes which seems capable to explain both positive and negative results based on a semiclassical model [90], but Tang et al. pointed out such model cannot account for the relatively large negative magnitude of the intrinsic DWMR they observed [89]. Actually, the intrinsic DWMR is difficult to measure, not only due to the very small resistant value, but also the requirement to carefully exclude the effects of anisotropic magnetoresistance (AMR), Lorentz MR and eddy-like current $[89,91]$. It is worth noted that, for domain wall in a Permalloy nanowire, the AMR with a negative value is dominant over all the other contributions [19].

Actually, both the uncertainty of DWMR values and the ambiguity of theoretical explanations stated above are only for wide domain walls, while for very sharp domain wall or ferromagnetic nanocontact, the conclusion will be clear and even universal [9294]. Unlike wide domain walls, the ballistic transport regime instead of diffuse transport regime will be considered for sharp domain walls, and the ballistic magneto-resistivity could be as high as $300 \%$ at room temperature which can be explained (based on a linear response theory) via scattering by the sharp domain wall constrained within the nanocontact [93]. Almost all the reported experimental measurements [92, 95, 96] and theoretical analysis $[93,97-100]$ demonstrate the same conclusion by showing the same universal scenery, the sharper the domain wall, the higher the magneto-resistivity, i.e., nanocontact actually can act as a spin valve, which makes sense since spin-dependent 
scattering of incident electrons by sharper domain wall is more intensive than wide wall within which incident electrons can easily track local magnetizations. Besides, the magneto-resistivity also depends on current polarization $P$ which is determined by the density of states of majority and minority channels at Fermi level. Highly spin-polarized current (with large $P$ ) can enhance the spin-dependent scattering by domain wall to exhibit a strong spin-valve effect. An extreme case is the half-metal for which the minority channel is empty at Fermi level and then $P=1$ representing the fully spinpolarized current, so that half-metals play a very important role in spintronics. Therefore, huge DWMR can be expected for very sharp or absolutely abrupt domain wall in some certain ferromagnetic materials, for instance, magneto-resistivity ratio as huge as up to $700 \%$ has been exhibited by a Ni-Ni nanocontact at room temperature [101], which can perform as a very good spin filter. Potential applications in spintronics can be proposed for domain walls with high DWMR which may be treated as the building block of novel MRAM.

Once the nanocontacts shrink further, until only a few atoms or even one single atom are bridged between two magnetic domains, the MR will be quantized, which might lead to atomic-size devices in nanospintronics. Actually, DWMR for atomic-size contacts have also been investigated and quantized MR has also been observed [80, 102]. Extensive $a b$ initio calculations using tight-binding model [103-105], nonequilibrium Green's function (NEGF) formalism [106-108], or Korringa-Kohn-Rostoker (KKR) Green's function method [109-111] have been made for various ferromagnetic systems to study the atomic-size contact transportation properties or the tunneling magnetoresistance (TMR) effect. Therefore, for nanosized or atomic magnetic elements like molecular 
devices or atomic devices, ab initio studies play a very important role in discovering novel functional magnetic systems and predicting unique spintronic properties, for which it is still challenging to do in experiment. ab initio studies show that monoatomic chains of certain transition-metals like $\mathrm{Mn}, \mathrm{Fe}$, Co or Ni exhibit nearly half-metallic properties [112] and atomic chains of carbon-transition-metal compounds like $\mathrm{C}_{n} \mathrm{Cr}, \mathrm{C}_{n} \mathrm{Ti}$ or $\mathrm{C}_{n} \mathrm{Co}$ with specific $n$ exhibit fully half-metallic behavior with pronounced DWMR ratio [106, 113]. What's more, DWMR for freestanding atomic nanowires or atomic chains of $\mathrm{Fe}$, Co or Ni have been also calculated at an ab initio level [109]. All of those studies would open a new perspective in future nanospintronics devices.

\subsection{Magnetic Domain Walls in Thin Films, Nanowires and Atomic Chains}

Considering the great importance and potential applications of magnetic domain walls in magnetics, spintronics and magnonics, their properties and functionalities at three different scales, thin films, nanowires and atomic chains, will be studied by using micromagnetic simulations, theoretical analysis, numerical calculations and ab initio calculations in this dissertation.

In thin films, since the static and dynamic properties of domain walls usually have a great impact on domain nucleation, switching and stabilization, the fundamental properties like roughness, creep or depinning of domain wall under random pinning potentials and thermal fluctuations driven by magnetic field are computationally studied combined with relevant theoretical analysis in Sec. 3.1. Since substructures (or Bloch lines) are usually existed within domain walls and their density can also be modulated by a certain means, the fundamental static and dynamic properties of domain wall with 
substructures are also studied hereafter in Sec. 3.2 to demonstrate the effect of those dynamical topological defects (Bloch lines). As is similar with magnetic field, spinpolarized current can also provide a domain wall a driving force, the fundamental static and dynamic properties in the creep, depinning and flow regimes of domain wall with and without substructures driven by spin-polarized current should be also studied, which will be our future work. Before that, Bloch line dynamics and current induced Bloch line motion properties in ideal thin film without thermal fluctuations are studied in Sec. 3.3.

All the studies for domain walls in nanowires are presented in Chapter 4. For the ferromagnetic system, we choose the most widely investigated nanowire material, Permalloy, exhibiting very weak magnetocrystalline anisotropy, in the whole chapter except Sec. 4.5 in which cobalt nanowire is adopted. For domain walls in a planar Permalloy nanowire, we show transverse domain walls can exhibit broad potential applications in spintronics. In Sec. 4.1, we constructed novel nanometer transversedomain-wall-based logic elements including the data generator, NOT, AND and OR logic gates by combining both nanowire circuits loop and rotating magnetic field based on our micromagnetic simulations, which exhibits more advantages than the previously proposed submicrometer domain wall logic [34]. In Sec. 4.2, two devices based on $360^{\circ}$ domain wall (combination of two $180^{\circ}$ domain walls) are proposed, one is domain wall generator, and the other is domain wall shift register, and generation, storage and shifting of $360^{\circ}$ domain walls in planar magnetic nanowires driven by rotating magnetic field are computationally studied. In Sec. 4.3, spin-polarized current instead of magnetic field is applied to the domain wall pile-up, and altering critical depinning current via domain wall pile-up in magnetic nanowires is investigated by our simulations. In Sec. 4.4, current 
induced domain wall depinning and transportation through planar nanowire circular geometry by spin transfer torques and spin-orbit torques are theoretically analyzed and numerically calculated from the micromagnetic model. In Sec. 4.5, magnetic vortex racetrack memory is proposed by combining both the concepts of vortex domain wall and racetrack memory implemented in the Co nanowire with perpendicular magnetocrystalline anisotropy. In the future, the static and dynamic properties of domain wall under DMI effect driven by spin-polarized current including spin-orbit torque effect will be computationally studied.

The properties of atomic domain wall in atomic chains are studied in Chapter 5. Since the magnetocrystalline anisotropy energy is a key factor in one-dimensional atomic chain system, Ni monoatomic chain is chosen as our ferromagnetic atomic chain system due to its high magnetocrystalline anisotropy energy. At atomic scale, the phenomenological micromagnetic model is failed, therefore, both $a b$ initio studies and spin dynamics simulations are carried out in this chapter. All the relevant magnetic parameters are calculated at $a b$ initio density function theory level at zero temperature, with the magnetic parameters at finite temperature calculated based on a DFT approach to the electronic free energy. Using an atomistic model combined with ab initio studies, a truly magnetic domain wall structure and the single domain switching process are investigated by our spin dynamics simulations. Similar studies on the 4-d transition-metal with high magnetocrystalline anisotropy energy deposited on proper substrate will be our future work, and other ab initio programs like SMEAGOL may be used. 


\section{Chapter 2. Computational Methods}

\subsection{Micromagnetic Modeling}

In the micromagnetic model, the evolution of the magnetization field is described by the Landau-Lifshitz-Gilbert equation [114]

$$
\frac{d \mathbf{m}(\mathbf{r})}{d t}=\gamma \mathbf{H}_{\mathrm{eff}}(\mathbf{r}) \times \mathbf{m}(\mathbf{r})+\alpha \mathbf{m}(\mathbf{r}) \times \frac{d \mathbf{m}(\mathbf{r})}{d t},
$$

where $\mathbf{r}$ is the spatial position vector, $\mathbf{m}(\mathbf{r})$ is the magnetization directional unit vector field, $\gamma$ is the gyromagnetic ratio, $\alpha$ is the damping parameter, and $\mathbf{H}_{\text {eff }}(\mathbf{r})$ is the effective magnetic field.

Although the spontaneous magnetization is contributed by both spin moment and orbital moment, only spin moment dominantly contributes the magnetization, since orbital moment is largely quenched by crystal field. The driving force of magnetization processes, $\mathbf{H}_{\text {eff }}(\mathbf{r})$, is calculated by variational derivative of the total system free energy $E$ with respect to the magnetization,

$$
\mathbf{H}_{\mathrm{eff}}(\mathbf{r})=-\frac{\delta E}{M_{s} \delta \mathbf{m}(\mathbf{r})}
$$

where $M_{s}$ is the saturation magnetization. The total system free energy $E$ is given by

$$
E=E_{\text {exch }}+E_{\text {ani }}+E_{\text {mag }}+E_{\text {ext-mag }}+E_{\text {elast }}+E_{\text {ext-elast }},
$$

where $E_{\text {exch }}$ represents the exchange energy, $E_{a n i}$ the magnetocrystalline anisotropy

energy, $E_{m a g}$ the magnetostatic energy, $E_{\text {elast }}$ the elastic energy, $E_{\text {ex-mag }}$ the external magnetic energy, and $E_{\text {ex-elast }}$ the external elastic energy. 
The exchange energy originated from the Heisenberg model dealing with the triplet state (parallel spins) and singlet state (antiparallel spins) [64] is the very heart of magnetic order origin. In the phenomenological model, it is given by

$$
E_{\text {exch }}=A \int[\operatorname{grad} \mathbf{m}(\mathbf{r})]^{2} d^{3} r
$$

where $A$ is the exchange stiffness constant, which can be used to determine the Curie temperature of ferromagnetic materials. The anisotropy energy is originated from spinorbit interaction effect, and its magnitude as well as its function form is dependent of specific lattice structure of certain ferromagnetic materials. Usually, most $3 \mathrm{~d}$ transitionmetals often have weak magnetocrystalline anisotropy energy, while most $4 \mathrm{~d}$ and $5 \mathrm{~d}$ transition-metals have strong magnetocrystalline anisotropy effect. For uniaxial ferromagnetic systems, the anisotropy energy term is usually described by the following form

$$
E_{a n i}(\mathbf{r})=\int\left(K_{1}\left\{1-[\mathbf{p}(\mathbf{r}) \cdot \mathbf{m}(\mathbf{r})]^{2}\right\}+K_{2}\left\{1-[\mathbf{p}(\mathbf{r}) \cdot \mathbf{m}(\mathbf{r})]^{2}\right\}^{2}\right) d^{3} r
$$

where $K_{1}$ and $K_{2}$ are uniaxial magnetocrystalline anisotropy constants, and $\mathbf{p}(\mathbf{r})$ is the easy axis determined by the spin-orbit coupling. The magnetostatic energy can be presented in reciprocal space using the Fourier transform of the magnetization field,

$$
E_{m a g}=\frac{1}{2} M_{s}^{2} \int \frac{d^{3} k}{(2 \pi)^{3}} 4 \pi \frac{|\mathbf{k} \cdot \mathbf{m}(\mathbf{k})|^{2}}{k^{2}}-\frac{2 \pi}{3} V M_{s}^{2} .
$$

The external field energy which is due to Zeeman interactions is simply given by

$$
E_{e x t}=-M_{s} \int \mathbf{H}^{\mathrm{ext}} \cdot \mathbf{m}(\mathbf{r}) d^{3} r
$$


where $\mathbf{H}^{\text {ext }}$ is the external magnetic field applied on the magnetizations. Magnetostriction is also related to the spin-orbit interaction effect and it arises from the strain dependence of the magnetocrystalline anisotropy constants. The elastic energy due to the magnetostrictive effect is

$$
E_{\text {elast }}=\frac{1}{2} \int \frac{d^{3} k}{(2 \pi)^{3}}\left[C_{i j k l}-n_{p} C_{i j p q} \Omega_{q r}(\mathbf{n}) C_{k l r s} n_{s}\right] \tilde{\varepsilon}_{i j}^{0}(\mathbf{k}) \tilde{\varepsilon}_{k l}^{0^{*}}(\mathbf{k}),
$$

where $\varepsilon_{i j}^{0}(\mathbf{r})=\alpha_{p q r s} m_{k}(\mathbf{r}) m_{l}(\mathbf{r}), \alpha_{p q r s}$ is the magnetostrictive coefficient tensor, and $\Omega_{i j}(\mathbf{n})$ is Green function tensor inverse to $\Omega_{i j}^{-1}(\mathbf{n})=C_{i k j l} n_{k} n_{l}$. The external elastic energy is given by

$$
E_{e x t}=-\int \boldsymbol{\sigma}^{\mathrm{ext}}: \boldsymbol{\varepsilon}^{0}(\mathbf{r}) d^{3} r
$$

where $\boldsymbol{\sigma}^{\mathrm{ext}}$ is the external stress.

The total system energy is a functional of $\mathbf{m}(\mathbf{r})$, and is evaluated by as a sum of the above six energy contributions,

$$
\begin{aligned}
E & =\int\left(A[\operatorname{grad} \mathbf{m}(\mathbf{r})]^{2}+K_{1}\left\{1-[\mathbf{p}(\mathbf{r}) \cdot \mathbf{m}(\mathbf{r})]^{2}\right\}+K_{2}\left\{1-[\mathbf{p}(\mathbf{r}) \cdot \mathbf{m}(\mathbf{r})]^{2}\right\}^{2}\right) d^{3} r \\
& +\frac{1}{2} M_{s}^{2} \int \frac{d^{3} k}{(2 \pi)^{3}} 4 \pi \frac{|\mathbf{k} \cdot \mathbf{m}(\mathbf{k})|^{2}}{k^{2}}-\frac{2 \pi}{3} V M_{s}^{2}-M_{s}^{2} \int \mathbf{h}_{e x t} \cdot \mathbf{m}(\mathbf{r}) d^{3} r \\
& +\frac{1}{2} \int \frac{d^{3} k}{(2 \pi)^{3}}\left[C_{i j k l}-n_{p} C_{i j p q} \Omega_{q r}(\mathbf{n}) C_{k l r s} n_{s}\right] \tilde{\varepsilon}_{i j}^{0}(\mathbf{k}) \tilde{\varepsilon}_{k l}^{0 *}(\mathbf{k})-\int \boldsymbol{\sigma}^{\mathrm{ext}}: \boldsymbol{\varepsilon}^{0}(\mathbf{r}) d^{3} r
\end{aligned}
$$

When spin-polarized current is applied, the evolution of magnetization field is described by the modified Landau-Lifshitz-Gilbert equation which includes the conventional spin transfer torques $[3,8,51]$,

$$
\frac{d \mathbf{m}}{d t}=\gamma \mathbf{H} \times \mathbf{m}+\alpha \mathbf{m} \times \frac{d \mathbf{m}}{d t}-(\mathbf{u} \cdot \nabla) \mathbf{m}+\beta \mathbf{m} \times[(\mathbf{u} \cdot \nabla) \mathbf{m}],
$$


where $\mathbf{u}$ is the electron motion velocity vector and $\beta$ the nonadiabatic spin-transfer torque coefficient. The last two terms are adiabatic and nonadiabatic spin-transfer torque terms, respectively. The electron motion velocity vector proportional to the electrical current density $\mathbf{J}$ is determined by $\mathbf{u}(x)=-\mathbf{J}(x) P g \mu_{B} /\left(2 e M_{s}\right)$ where $P$ is the polarization rate of the current and the factor $g \mu_{B} /\left(2 e M_{s}\right)=7 \times 10^{-11} \mathrm{~m}^{3} / \mathrm{C}$ for Permalloy [51]. Usually, the magnitude of $\beta$ is much less than unity and comparable to $\alpha$, the values for both $\alpha$ and $\beta$ can be modulated by certain type of doping [115].

When interface-derived spin-orbit torques induced by Rashba effect and spin Hall effect are taken into account, the modified Landau-Lifshitz-Gilbert equation becomes $[51,69,72,76]$

$$
\begin{aligned}
\frac{\partial \mathbf{m}}{\partial t}= & -\gamma \mathbf{m} \times\left(\mathbf{H}_{\text {eff }}+\mathbf{H}_{R}\right)+\alpha \mathbf{m} \times \frac{\partial \mathbf{m}}{\partial t} \\
& -(\mathbf{u} \cdot \nabla) \mathbf{m}+\beta \mathbf{m} \times[(\mathbf{u} \cdot \nabla) \mathbf{m}]-\alpha_{S H} \mathbf{m} \times[\mathbf{m} \times \hat{\sigma}],
\end{aligned}
$$

where $\mathbf{H}_{R}$ is the Rashba effective field, $\alpha_{S H}$ the parameter determining the amplitude of spin Hall effect, and $\hat{\sigma}$ the unit vector denoting the spin Hall effect induced spin direction. The Rashba effective field is given by

$$
\mathbf{H}_{R}(x)=\frac{\alpha_{R} P}{\mu_{0} \mu_{B} M_{s}} \hat{z} \times \mathbf{J}(x)=\frac{2 e \alpha_{R}}{g \mu_{B}^{2}} \mathbf{u}(x) \times \hat{z}=-\frac{2 e \alpha_{R} u}{g \mu_{B}^{2}} \hat{\sigma},
$$

where $\alpha_{R}$ is the Rashba parameter. The spin Hall effect related parameter $\alpha_{S H}$ can be given by

$$
\alpha_{S H}=\frac{\gamma\left({ }^{\prime} \theta_{S H} J\right.}{2 e \mu_{0} M_{s} L_{z}},
$$


where $L_{z}$ is the ferromagnetic layer thickness and $\theta_{S H}$ is the spin-Hall angle whose value can be either positive or negative determined by the substrate layer material $[70,71]$.

\subsection{Ab Initio Method}

In principle, the electronic structure of atoms, molecules and solids can be exactly calculated and the physical and chemical properties can be thereby predicted by solving many-particle Schrödinger equation. However, analytical or numerical solutions are possible only for very simple systems. Thanks to the density function theory (DFT) introduced in 1960s for which a lot of reasonable assumptions and approximations are used, the parameter-free $a b$ initio calculations can be made. DFT is presently the most successful approach to compute the electronic structure of matter and predict a great variety of properties for systems of atoms, molecules and solids. Since there has been many review articles [116-119] and books [120-122] talking about DFT in detail, only a very brief introduction to DFT and the key milestones to develop DFT is presented herein. In order to approximate the solution of many-particle Schrodinger equation, Hartree-Fock approximation (or self-consistent field (SCF) method) based on BornOppenheimer approximation is proposed to approximate the many-electron wave function as a product of single-particle functions or Slater determinant. In the HartreeFock approximation, the mean field approximation is implied, i.e., effects of electron correlation are completely neglected, which leads to the post-Hartree-Fock approximation including the repulsions between electrons. Examples of post-Hartree-Fock approximation include configuration interaction $(\mathrm{CI})$, quadratic configuration interaction (QCI) and so on. Although it is still challenging to extend to solid systems, the (post- 
Hartree-Fock approximation is an indispensable benchmark in molecular physics. An alternate approach is to use electron density instead of wave function as the central quantity, resulting in the Thomas-Fermi model which is the first density functional theory. The Hohenberg-Kohn theorem demonstrates that the electron density uniquely determines the Hamiltonian operator and thus all the properties of the system. After that, Kohn-Sham replaced the original many-particle problem with an independent electron problem by proposing the Kohn-Sham equations that can be solved, which requires approximations for the exchange-correlation energy. If the exact forms of the exchangecorrelation energy were known, the Kohn-Sham strategy would lead to the exact energy, and for that issue, the local density approximation (LDA) and the generalized gradient approximation (GGA) are made. Lots of ab initio computer programs based on DFT have been developed, such as Vienna Ab initio simulation package (VASP), Spanish Initiative for Electronic Simulations with Thousands of Atoms (SIESTA), ABINIT, Quantum ESPRESSO and so on. For our magnetic system studies, we adopt VASP [123, 124] which uses a plane wave basis set to carry out our calculations.

For spin-polarized calculations, the spin density function theory (SDFT) is used. Fully unconstrained noncollinear magnetism has been successfully implemented within VASP [125], and a brief introduction is presented herein. Compared to DFT, the wavefunction becomes a spinor

$$
|\Phi\rangle=\left(\begin{array}{c}
\left|\Psi^{\uparrow}\right\rangle \\
\left|\Psi^{\downarrow}\right\rangle
\end{array}\right),
$$

and the electron density becomes a $2 \times 2$ matrix, 


$$
\begin{aligned}
n^{\alpha \beta}(\mathbf{r}) & =\sum_{n} f_{n}\left\langle\Psi_{n}^{\beta} \mid \mathbf{r}\right\rangle\left\langle\mathbf{r} \mid \Psi_{n}^{\alpha}\right\rangle \\
& =\left[n_{T r}(\mathbf{r}) \delta_{\alpha \beta}+\mathbf{m}(\mathbf{r}) \cdot \boldsymbol{\sigma}^{\alpha \beta}\right] / 2,
\end{aligned}
$$

where $f_{n}$ is the orbital occupation number, $\boldsymbol{\sigma}$ the Pauli spin matrices, and $\mathbf{m}(\mathbf{r})=\sum_{\alpha \beta} n^{\alpha \beta}(\mathbf{r}) \cdot \boldsymbol{\sigma}^{\alpha \beta}$ is the magnetization density.

Therefore, the spin-polarized Kohn-Sham equation becomes

$$
\sum_{\beta} H^{\alpha \beta}\left|\Psi_{n}^{\beta}\right\rangle=\varepsilon_{n} S^{\alpha \alpha}\left|\Psi_{n}^{\alpha}\right\rangle,
$$

with the Hamiltonian matrix given by

$$
H^{\alpha \beta}=-\frac{1}{2} \Delta \delta_{\alpha \beta}+V_{e x t}(\mathbf{r}) \delta_{\alpha \beta}+\int d \mathbf{r}^{\prime} \frac{n_{T r}\left(\mathbf{r}^{\prime}\right)}{\left|\mathbf{r}-\mathbf{r}^{\prime}\right|} \delta_{\alpha \beta}+V_{x c}^{\alpha \beta}[\ddot{n}(\mathbf{r})](\mathbf{r}),
$$

where $V_{e x t}(\mathbf{r})$ is the effective one-electron potential and $V_{x c}^{\alpha \beta}[\vec{n}(\mathbf{r})]$ is the exchangecorrelation potential. According to the $2 \times 2$ matrix in Eq. (2.2.4), $H^{\alpha \alpha}$ and $H^{\beta \beta}$ are the diagonal elements while $V_{x c}^{\alpha \beta}$ and $V_{x c}^{\beta \alpha}$ are nondiagonal elements. In the absence of those nondiagonal elements, the noncollinear case will fall into the collinear case.

When considering spin-orbit interaction, the spin directions will be linked to the crystalline structure and the magneto-crystalline anisotropy can be calculated. The spinorbit Hamiltonian is given by

$$
H_{S O I}^{\alpha \beta}=\frac{\mathfrak{n}^{2}}{\left(2 m_{e} c\right)^{2}} \sum_{i, j}\left\langle\phi_{i}\left|\frac{1}{r} \frac{d V_{\text {spher }}}{d r}\right| \phi_{j}\right\rangle\left|\vec{p}_{i}\right\rangle \vec{\sigma}_{\alpha \beta} \cdot \vec{L}_{i j}\left\langle\tilde{p}_{j}\right|
$$

where $\widetilde{L}_{i j}$ is angular momentum.

For the noncollinear calculations, spin spiral computation module is also implemented in VASP. The magnetization for spin spiral case is given by 


$$
\mathbf{m}(\mathbf{r}+\mathbf{R})=\left(\begin{array}{c}
m_{x}(\mathbf{r}) \cos (\mathbf{q} \cdot \mathbf{R})-m_{y}(\mathbf{r}) \sin (\mathbf{q} \cdot \mathbf{R}) \\
m_{x}(\mathbf{r}) \sin (\mathbf{q} \cdot \mathbf{R})+m_{y}(\mathbf{r}) \cos (\mathbf{q} \cdot \mathbf{R}) \\
m_{z}(\mathbf{r})
\end{array}\right)
$$

where $\mathbf{q}$ is the spin spiral vector and $\mathbf{R}$ is the spin spiral wave length. Then, the wavefunction spinor becomes

$$
\left(\begin{array}{c}
\Psi_{\mathbf{k}}^{\uparrow} \\
\Psi_{\mathbf{k}}^{\downarrow}
\end{array}\right)=\left(\begin{array}{cc}
e^{-i \mathbf{q} \cdot \mathbf{R} / 2} & 0 \\
0 & e^{+i \mathbf{q} \cdot \mathbf{R} / 2}
\end{array}\right)\left(\begin{array}{c}
\Psi_{\mathbf{k}}^{\uparrow}(\mathbf{r}-\mathbf{R}) \\
\Psi_{\mathbf{k}}^{\downarrow}(\mathbf{r}-\mathbf{R})
\end{array}\right),
$$

and the $2 \times 2$ matrix becomes

$$
\left(\begin{array}{cc}
H^{\alpha \alpha} & V_{x c}^{\alpha \beta} e^{-i \mathbf{q} \cdot \mathbf{r}} \\
V_{x c}^{\beta \alpha} e^{+i \mathbf{q} \cdot \mathbf{r}} & H^{\beta \beta}
\end{array}\right) .
$$

The detailed introduction on the spin spiral calculations can be found in Ref. [126, 127]. 


\section{Chapter 3. Magnetic Domain Walls in Thin Films}

\subsection{Domain Wall Creep in an Ultrathin Magnetic Film with a Driving Field near the Depinning Force}

\subsubsection{Introduction}

The creep motion for a d-dimensional elastic manifold moving in a $(d+1)$ dimensional random media can be found in many physical systems. Examples include vortices in type-II superconductors [128], charge density waves [129], ferroelectric domain walls [130, 131] and ferromagnetic domain walls [132-135]. The case of $d=1$ relates to an elastic string moving in a random interface. Domain wall in ultrathin $\mathrm{Pt} / \mathrm{Co} / \mathrm{Pt}$ film which can be treated as a one-dimensional domain wall line is one example of such elastic strings. Both the experimental[134-137] and computational [138-140] studies have been made to better understand the dynamics of such domain wall line with driving magnetic field in the creep regime, as well as in the depinning and flow regimes. For the creep regime, however, most researches focused on the region where the external magnetic field is much smaller than the depinning force, i.e., $H \ll H_{c}$, and the domain wall creep behavior in the region where the magnetic field is smaller than but rather close to the depinning force, i.e., $H<H_{c}$, was seldom investigated. In the creep regime, domain wall motion velocity obeys an Arrhenius law $v=v_{0} \exp (-U / k T)$, where $U=U_{c}\left(H_{c} / H\right)^{\mu}$ with a universal constant exponent $\mu=(2 \zeta-1) /(2-\zeta)$ for domain wall line $(\zeta=2 / 3)$ with $H \ll H_{c}$, while $U=U_{c}\left(1-H / H_{c}\right)^{\alpha}$ with the exponent $\alpha$ not determined yet for domain wall line with $H<H_{c}$. Although there is no exact theory to determine the creep exponent $\alpha$ for $H<H_{c}$, there are actually three possible values existed. The first one is $\alpha=$ 
$\beta / \psi=2.22$ which is obtained just through simple phenomenological interpolation between regimes of creep, depinning and flow, [141] where $\beta$ is the depinning exponent and $\psi$ is the thermal rounding exponent with both of the values determined as $1 / 3$ and 0.15 , respectively [132]. The second one is $\alpha=1$ which is calculated using dynamical renormalization group theory based on the short range correlated random potential or pointlike impurities and independent of dimensionality, [139] and is in accordance with Anderson's original theory of classical creep [142]. The third one is $\alpha=(6-\mathrm{d}) / 4=5 / 4$ which is based on washboard potential and depends on the dimensionality. [128, 143]

The exact value of $\alpha$ strongly depends on the determination of depinning force $H_{c}$. The exact value of $H_{c}$, actually, can be obtained from the depinning regime, in which the velocity-field relationship is predicted[138] by $v \propto\left(H / H_{c}-1\right)^{\beta}$, where the universal depinning exponent $\beta \approx 1 / 3$ is theoretically expected based on numerical simulations $[144,145]$ and has been further supported by recent experiments [132]. After determining the depinning force $H_{c}$, creep exponent $\alpha$ can be then obtained from the velocity-filed relationship $v \propto \exp \left\{-U_{c}\left(1-H / H_{c}\right)^{\alpha} / k_{B} T\right\}$. In this section, we use phase field model and micromagnetic simulations to obtain the domain wall velocities with a serious of external magnetic fields in the whole range, and then calculate the creep exponent $\alpha$, before which the depinning force is predetermined in the same way as in Ref. [132].

\subsubsection{Micromagnetic Model}

The evolution of magnetization is described by the Landau-Lifshitz-Gilbert equation $[51,146]$ 


$$
\frac{\partial \mathbf{m}}{\partial t}=-\gamma \mathbf{m} \times\left(\mathbf{H}_{\mathrm{eff}}+\mathbf{H}_{\mathrm{th}}\right)+\alpha \mathbf{m} \times \frac{\partial \mathbf{m}}{\partial t},
$$

where $\mathbf{m}$ is a unit vector of magnetization, $\gamma$ the gyromagnetic constant, $\alpha$ the Gilbert damping constant, $\mathbf{H}_{\text {eff }}$ the effective magnetic field $\mathbf{H}_{\text {eff }}=-\delta E /\left(M_{s} \delta \mathbf{m}\right)$, and $\mathbf{H}_{\text {th }}$ the Gaussian distributed random fluctuating thermal field satisfying [146]

$$
\begin{gathered}
\left\langle H_{t h, i}(\mathbf{r}, t)\right\rangle=0, \\
\left\langle H_{t h, i}(\mathbf{r}, t) H_{t h, j}\left(\mathbf{r}^{\prime}, t^{\prime}\right)\right\rangle=2 D_{t h} \delta_{i j} \delta\left(\mathbf{r}-\mathbf{r}^{\prime}\right) \delta\left(t-t^{\prime}\right),
\end{gathered}
$$

where $D_{t h}$ is temperature dependent of strength of the thermal field given by

$$
D_{t h}=\frac{\alpha k_{B} T}{\gamma \mu_{0} M_{s} l^{3}}
$$

where $k_{B}$ is the Boltzmann constant, $T$ is the temperature, and $l$ the simulation grid size. For the parameters used in our simulations, we adopt the experimental measured values for domain wall in $0.6 \mathrm{~nm}$ thick ultrathin $\mathrm{Pt} / \mathrm{Co} / \mathrm{Pt}$ film with perpendicular anisotropy [134]: saturation magnetization $M_{s}=1130 \mathrm{emu} / \mathrm{cm}^{3}$, uniaxial perpendicular magnetocrystalline anisotropy constant $K_{u}=1.25 \times 10^{7} \mathrm{erg} / \mathrm{cm}^{3}$, exchange constant $A=1.6 \times 10^{-6} \mathrm{erg} / \mathrm{cm}$, and Gilbert damping constant $\alpha=0.3$. The statistical defects are realized by introducing a fluctuation of magnetocrystalline anisotropy obeying Gaussian distribution with an amplitude of $\delta K / K_{u} \approx 0.055$, and that type of defects is reported to be the main pinning source in ultrathin $\mathrm{Pt} / \mathrm{Co} / \mathrm{Pt}$ film with perpendicular anisotropy. [147] Large system size of $8.1 \mu \mathrm{m} \times 0.65 \mu \mathrm{m}$ with one grid size of $4.5 \mathrm{~nm}$ is used to reduce the long range interaction between neighboring domain walls. In our simulations, actually, 
the distance between two neighbor domain walls is as large as $4 \mu \mathrm{m}$, resulting in an ignorable maximum stray field range as small as $\pm 10^{-3} M_{s}$.

\subsubsection{Results and Discussion}

Unlike the ideal domain wall, the domain wall in a thin film with statistical defects pinning sites will become rough due to the competition between curved domain wall energy and pinning sites energy barrier. The random fluctuated thermal field with amplitude corresponding to the room temperature is applied in our simulation process, which makes the curved domain wall line profile different from time to time, and such a rough domain wall line is shown by the inset in Fig. 3.1 (a). The domain wall roughness can be characterized by a wandering exponent $\zeta$ which can be obtained from the transverse displacement correlation function obeying a scaling law [128]:

$$
\left\langle[u(x+L)-u(x)]^{2}\right\rangle \propto u_{0}^{2}\left(\frac{L}{L_{c}}\right)^{2 \zeta}
$$

where $u_{0}$ is a transverse scaling parameter and $L_{c}$ a characteristic collective pinning length. The wandering exponent $\zeta$ can be obtained by making a linear fitting as shown in Fig. 3.1 (a), and its value $\zeta=0.666$ is consistent with the proved universal value $\zeta=2 / 3$ for one-dimensional elastic string in random media [128]. Therefore, the value of creep exponent $\mu$ which depends on $\zeta$ is also determined, i.e., a universal creep exponent $\mu=$ $1 / 4$ will be used for the analysis of velocity-field relationship in the creep regime for $H \ll H_{c}$. 

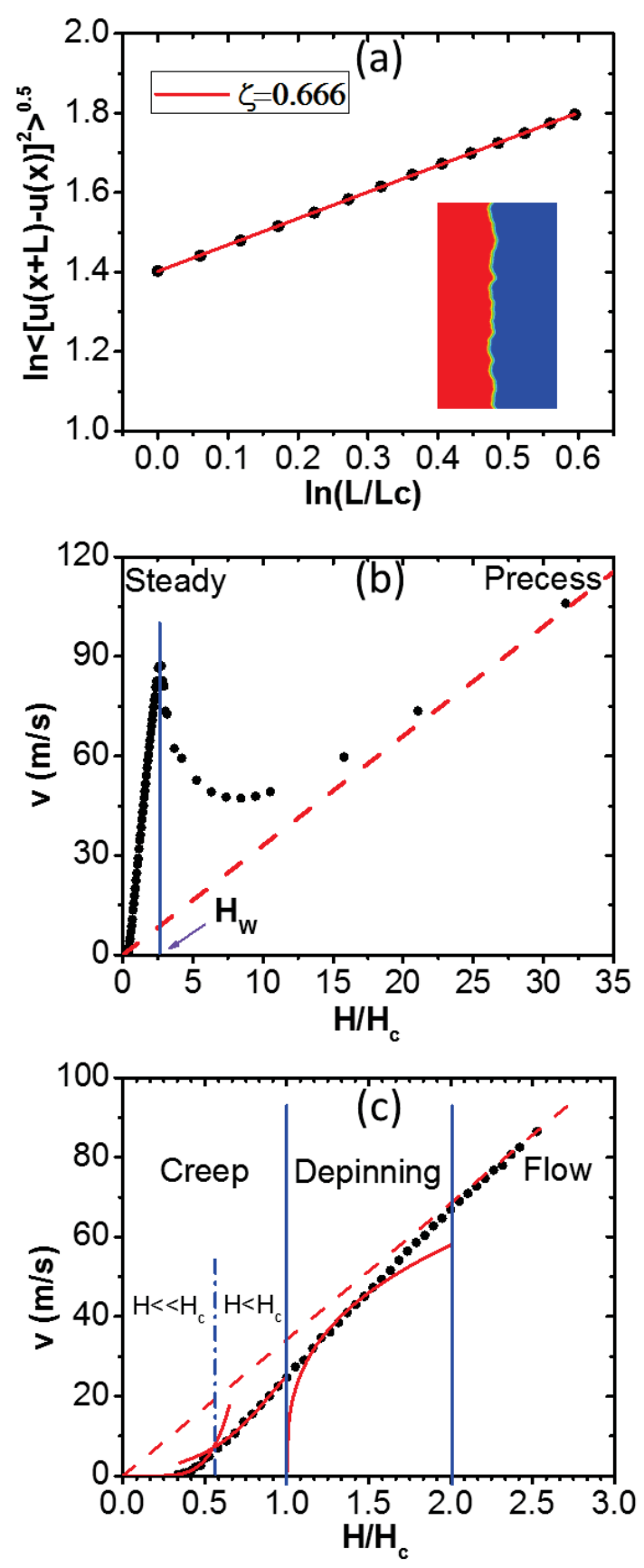

Figure 3.1 (a) Linear fitting of $\ln \left\langle[u(x+L)-u(x)]^{2}\right\rangle^{1 / 2}$ vs. $\ln \left(L / L_{c}\right)$ for the domain wall line in ultrathin film with random pinning potential and thermal field as shown by the inset. (b) Whole range of domain wall velocity $v$ under external magnetic field $H$ normalized by $H_{c}=0.0095 M_{s}$. Walker field $H_{W} \approx 2.5 H_{c}$, below which is the steady linear flow regime, while farther beyond which is the precessional linear flow regime. (c) Velocity-field relationship within the steady linear flow regime. Solid red curves are the fitting curves, dashed red line is the fitting curve. 
The rough domain wall line will be moved under external magnetic field, and then the average motion velocity can be measured. For experimental observations [134], however, there is only one linear flow regime observed for domain wall moving in ultrathin $\mathrm{Pt} / \mathrm{Co} / \mathrm{Pt}$ film, which is explained by that the depinning force $H_{c}$ is larger than the Walker field $H_{W}$, i.e., only the precessional linear flow regime is experimentally observed. Our choice for the anisotropy fluctuation amplitude, i.e., $\delta K / K_{u} \approx 0.055$, which is used to introduce pinning effect, makes the depinning force $H_{c}$ smaller than the Walker field $H_{W} \approx 2.5 H_{c}$, and hence both the steady and precessional linear regimes can be observed, which is shown in Fig. 3.1 (b). Therefore, all the creep, depinning and flow regimes will fall into the steady linear flow regime in our simulations as shown in Fig. 3.1 (c), which is different from the experimental case. The velocity in these two linear flow regimes can be fitted very well using $v=m\left(H / H_{c}\right)$, where $m$ is the domain wall mobility whose values are $34.2 \mathrm{~m} / \mathrm{s}$ and $3.3 \mathrm{~m} / \mathrm{s}$ in the steady linear flow regime and the precessional linear flow regime, respectively, based on our micromagnetic simulation results in Fig. 3.1 (b) and (c). Those values are consistent with the case of ideal domain wall in the thin film without any pinning defects or thermal fluctuations.

The depinning force $H_{c}$ can be determined from the velocity-field relationship in the depinning regime. In that regime, we use the same way as in Ref. [132] to obtain $H_{c}$, i.e., a serious of possible test values of $H_{c}$ are used to fit the velocity-field relationship $v \propto\left(H / H_{c}-1\right)^{\beta}$ to obtain the corresponding $\beta$, and only the depinning force corresponding to $\beta=1 / 3$ occupies the exact value of $H_{c}$, and all the test cases as well as the final result, $H_{c}=0.0095 M_{s}$, are shown in Fig. 3.2 (a). In addition, for the zero- 
temperature depinning case, our simulation results indicate a depinning force $H_{c}$ in the range of $\left(0.009 M_{s}, 0.010 M_{s}\right)$, which supplies a further support for the above value. The curve using the obtained value $H_{c}=0.0095 M_{s}$ as well as $\beta=1 / 3$ is plot in Fig. 3.1 (c) in the depinning regime. It is noted that such a depinning relationship is valid only for a small field range in the middle of the depinning regime, while for the field close to $H_{c}$ or near the flow regime that relationship becomes invalid.

After determining the depinning force $H_{c}$, the creep exponent $\alpha$ for $H<H_{c}$ can be obtained through fitting the velocity-field relationship $v \propto \exp \left\{-U_{c}\left(1-H / H_{c}\right)^{\alpha} / k_{B} T\right\}$. The linear fitting is shown in Fig. 3.2 (b) with $\alpha=1.24$ for $H_{c}=0.0095 M_{s}$. The small error of ' \pm 0.05 ' is arisen from the fitting results using depinning force $H_{c}$ in the maximum range of $\left(0.009 M_{s}, 0.010 M_{s}\right)$, i.e., $H_{c}=0.0095 \pm 0.0005 M_{s}$. It is obvious that the creep exponent for $H<H_{c}, \alpha=1.24 \pm 0.05$, based on our simulation results is pretty close to $5 / 4$, the value theoretically calculated based on washboard potential model, and even the maximum error is still unable to bring it to the other two possible values, $\alpha$ $=1$ based on the short range correlated random potential or pointlike impurities and $\alpha=$ 2.2 based on simple phenomenological interpolation between regimes. The curve using the obtained value $\alpha=1.24$ as well as $H_{c}=0.0095 M_{s}$ is plot in Fig. 3.1 (c) in the creep regime with $H<H_{c}$, which fits very well with the original data. Thus, our micromagnetic simulation results support the exponent value of $\alpha=5 / 4$ based on washboard potential model. 

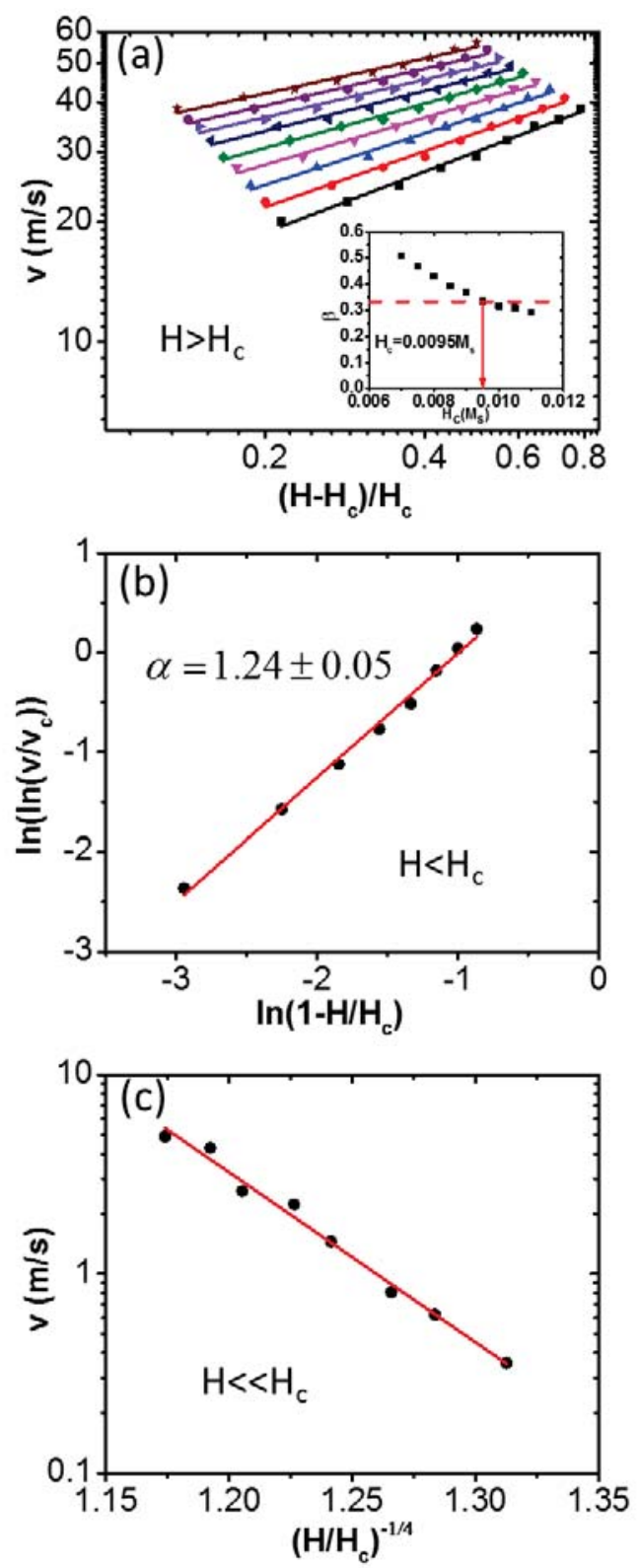

Figure 3.2 (a) Linear fittings for domain wall velocity with magnetic field according to $v \propto\left(H / H_{c}-1\right)^{\beta}$ with a serious of test depinning forces $H_{c}=0.007,0.0075,0.008,0.0085,0.009$, $0.0095,0.010,0.0105$ and $0.011 M_{s}$. The corresponding values of $\beta$ for those fittings are shown by the inset, with the depinning force $H_{c}=0.0095 M_{s}$ corresponding to $\beta=1 / 3$. (b) Linear fitting for domain wall velocity with magnetic field $\mathrm{H}<\mathrm{Hc}$ according to $v \propto \exp \left\{-U_{c}\left(1-H / H_{c}\right)^{\alpha} / k_{B} T\right\}$. The fitting result, i.e., the creep exponent is $\alpha=1.24 \pm 0.05$ with ' \pm 0.05 ' arising from $H_{c}=$ $0.0095 ` \pm 0.0005$ ' $M_{s}$. (c) Linear fitting for domain wall velocity with magnetic field $H \ll H_{c}$ according to $v \propto \exp \left\{-U_{c}\left(H_{c} / H\right)^{1 / 4} / k_{B} T\right\}$. 


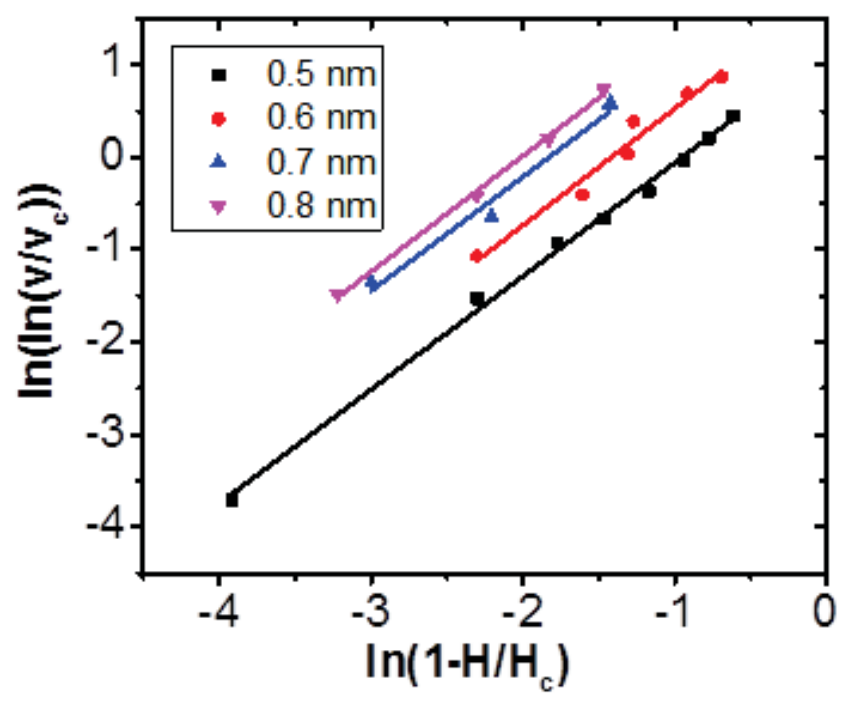

Figure 3.3 Linear fittings for domain wall velocity with magnetic field $H<H_{c}$ according to $v \propto \exp \left\{-U_{c}\left(1-H / H_{c}\right)^{\alpha} / k_{B} T\right\}$. All the data are from Ref. [132], experimental measurement results for ultrathin $\mathrm{Pt} / \mathrm{Co} / \mathrm{Pt}$ films with thickness of $0.5,0.6,0.7$ and $0.8 \mathrm{~nm}$.

Table 3.1 Creep exponents $\alpha$, pinning energies $U_{c}^{H \ll H_{c}}$ in $k_{B} T$ for $H \ll H_{c}$, pinning energies $U_{c}^{H<H_{c}}$ in $k_{B} T$ for $H<H_{c}$, as well as the ratios between the two types of pinning energies $U_{c}^{H<H_{c}} / U_{c}^{H<<H_{c}}$, obtained from our simulation results and the experimental results in Ref. [132] (marked by *).

\begin{tabular}{lcccc}
\hline & $\alpha$ & $\frac{U_{c}^{\left(H<<H_{c}\right)}}{k_{B} T}$ & $\frac{U_{c}^{\left(H<H_{c}\right)}}{k_{B} T}$ & $\frac{U_{c}^{\left(H<H_{c}\right)}}{U_{c}^{\left(H<H_{c}\right)}}$ \\
\hline Present work & 1.24 & 3.4 & 19.5 & 0.18 \\
$0.5 \mathrm{~nm}^{*}$ & 1.23 & 3.2 & 8.2 & 0.39 \\
$0.6 \mathrm{~nm}^{*}$ & 1.26 & 6.0 & 13.6 & 0.44 \\
$0.7 \mathrm{~nm}^{*}$ & 1.24 & 9.6 & 21.6 & 0.44 \\
$0.8 \mathrm{~nm}^{*}$ & 1.25 & 12.4 & 34.0 & 0.37 \\
\hline
\end{tabular}


What's more, the experimental data in Ref. [132] for which the depinning force $H_{c}$ is beyond the Walker field $H_{W}$ can also be used to obtain the creep exponent $\alpha$ in the same way as what we have done, although that was not considered by them. Such linear fittings are shown in Fig. 3.3 with all the data from Ref. [132] for ultrathin Pt/Co/Pt films with thickness of $0.5 \sim 0.8 \mathrm{~nm}$, and all the exponent values are listed in Table I. It is noted that those values are also around 5/4, i.e., the exponent value of $\alpha=5 / 4$ based on washboard potential model is also supported by experimental results.

Comparison of pinning energies $U_{c}$ for both creep regimes, $H<H_{c}$ and $H \ll H_{c}$, is also meaningful. All the energy values as well as their ratios are listed in Table 3.1. It is noted that the values obtained from our simulations are in the same order with those from experimental measurements. Since the pinning energies for both creep regimes originate from different physics, their values are not required to be equal. Actually, Table 3.1 shows that the pinning energies for $H<H_{c}$ are smaller than those for $H \ll H_{c}$. A brief analysis can be made for that. According to a phenomenological approach [128, 135], the total free energy for a domain wall line segment of length $L$ is

$$
F(u, L)=\frac{\varepsilon_{e l}}{2} \frac{u^{2}}{L}-\left(\gamma \xi^{2} L\right)^{1 / 2}-2 M_{s} H t L u,
$$

where $\varepsilon_{e l}$ is the string elasticity, $\gamma$ the disorder pinning strength, $t$ the film thickness, and $\xi$ is the character length of disorder potential. Therefore, the pinning energy calculated from Eq. (3.1.6) is

$$
U_{c}^{H<<H_{c}}=2\left(\varepsilon_{e l} \xi^{3} M_{s} t H_{c}\right)^{1 / 2} .
$$

In the creep regime of $H<H_{c}$, domain wall line is embedded in the washboard potential $V(u),[128,143]$ 


$$
V(u)=V_{0}\left(1-\cos k_{0} u\right)-2 M_{s} H t u,
$$

where $k_{0}$ is related to the washboard periodical length $u_{0}$ via $k_{0}=2 \pi / u_{0}$ and $V_{0}=M_{s} t H_{c} / k_{0}$ characterizes the pinning potential strength. The calculated pinning energy for $H<H_{c}$ is [128, 143]

$$
U_{c}^{H<H_{c}}=\frac{24}{5 \pi^{3 / 2}} 2^{1 / 4}\left(\varepsilon_{e l} u_{0}^{3} M_{s} t H_{c}\right)^{1 / 2} .
$$

Therefore, the ratio of the two pinning energies in Eq. (3.1.9) and (3.1.7) is

$$
\frac{U_{c}^{H<H_{c}}}{U_{c}^{H<<H_{c}}} \approx 0.5\left(\frac{u_{0}}{\xi}\right)^{3 / 2} .
$$

It can be noted from Eq. (3.1.10) that the ratio between the two pinning energies is related to the disorder lengths $u_{0}$ and $\xi$ for the two different types of pinning potential. According to Eq. (3.1.10), these ratios in Table I indicate a close value between $u_{0}$ and $\xi$, unlike vortices in superconductors [128] which suggests a much larger $u_{0}$, $u_{0}=2 \sqrt{6} \pi \xi$, based on the requirement that the length scales defined by the mean-toforce ratio are the same for both potentials, and hence a much larger $U_{c}^{H<H_{c}}$ which differs $U_{c}^{H \ll H_{c}}$ by an order of 10.

\subsubsection{Conclusion}

In conclusion, the dynamics of one-dimensional domain wall line in ultrathin $\mathrm{Pt} / \mathrm{Co} / \mathrm{Pt}$ film with perpendicular anisotropy in the creep, depinning and flow regimes with all of them within the steady linear flow region is investigated using our micromagnetic simulations. In order to obtain the creep exponent $\alpha$, the depinning force 
$H_{c}$ is predetermined by fitting the velocity-field relationship $v \propto\left(H / H_{c}-1\right)^{\beta}$ with $\beta=$ 1/3. The creep exponent $\alpha=1.24 \pm 0.05$ is determined by fitting $v \propto \exp \left\{-U_{c}\left(1-H / H_{c}\right)^{\alpha} / k_{B} T\right\}$ for $H<H_{c}$, pointing to the value $\alpha=5 / 4$ based on washboard potential model. The experimental data for ultrathin $\mathrm{Pt} / \mathrm{Co} / \mathrm{Pt}$ films with various thicknesses in Ref. [132] are also used by us to determine the relevant creep exponents, and all of the values are around 5/4, supplying a further support for the washboard model. The pinning energies $U_{c}$ for both creep regimes, $H<H_{c}$ and $H \ll H_{c}$, are briefly analyzed by a phenomenological approach for two distinct pinning potentials. Our analysis indicates that the ratio between the two pinning energies are dependent of the disorder lengths $u_{0}$ and $\xi$ for the two different types of pinning potentials, and both the simulations and experimental data indicate a close value between $u_{0}$ and $\xi$. The determination of creep exponent $\alpha$ can help better understand the dynamics for domain wall line with driving field near the depinning force, and it can also be extended to many other areas in physics. 


\subsection{Roughness, Creep, Depinning and Flow of Domain Wall Line with Substructures in an Ultrathin Magnetic Film}

\subsubsection{Introduction}

The statics and dynamics of elastic manifolds in random media is a very challenging problem which is relevant to many physical systems such as vortices in typeII superconductors [128], charge density waves [129], fluid invasion of porous media [148], ferroelectric domain walls [130, 131] and ferromagnetic domain walls [132-135]. Among them, one dimensional domain wall (DW) line, as an elastic string, in ultrathin magnetic film like $\mathrm{Pt} / \mathrm{Co} / \mathrm{Pt}$ film attracts much interest, and lots of investigations on the statics and dynamics of such elastic string have been made both experimentally [132-137, 149, 150] and computationally $[140,151,152]$. For statics, the roughness was experimentally studied, and reduced roughness or deroughening effect has been observed by dipolar repulsion [137] or by introducing linear defects [133, 136]for magnetic DW line. For dynamics, DW line behaviors in the creep, depinning and flow regimes have been studied [132-135]. In the creep regime with driving field much smaller than depinning field, i.e., $H<<H_{c}$, DW motion velocity obeys the Arrhenius law $v=v_{0} \exp (-U / k T)$, where $U=U_{c}\left(H_{c} / H\right)^{\mu}$ with a universal constant exponent $\mu=(2 \zeta-1) /(2-\zeta)=1 / 4$ for domain wall line whose roughness exponent is $\zeta=2 / 3$, while for that of driving field smaller than but rather close to the depinning field, i.e., $H<H_{c}, U=U_{c}\left(1-H / H_{c}\right)^{\alpha}$ with the exponent $\alpha=5 / 4$ which is theoretically calculated based on the washboard potential model [143] and has been computationally supported by our previous work in Sec. 3.1. In the depinning regime, the velocity-field 
relationship is predicted [138] by $v \propto\left(H / H_{c}-1\right)^{\beta}$, where the depinning exponent $\beta=1 / 3$ is expected by numerical simulations $[144,145]$ and has been further supported by recent experiments [132]. In the flow regime, the DW velocity is linearly related to the driving field, i.e., $v=m H$, where $m$ is the mobility which has different values in steady and precessional linear flow regimes.

In magnetic materials, DW substructures are commonly existed in many different forms, such as Bloch points, vortex/antivortex, and circular or cross Bloch lines. Those substructures are easy to be formed while somewhat hard to be removed. It is proposed that the existence of such substructures will affect drastically on the wall motion and domain nucleation [153]. On one hand, those substructures supply an additional freedom degree which allows them to move within DWs to reduce stored spin torques, and on the other hand, the small size of such substructures, which can be comparable to the defects in a thin film, makes them easily correlate with those defects, resulting in somewhat stronger pinning effect. Although much progress has been made for the statics and dynamics of DW line in ultrathin magnetic film, properties for DW line with substructures were rarely studied. Usually, the small size of substructures makes them difficult to be detected and modulated experimentally, what's more, it's also very hard for Mont Carlo simulations to implement so complicated substructures, both of which, therefore, make this problem very challenging and leave micromagnetic simulations perhaps the only method to study that challenging problem. For micromagnetic simulations, DW substructures can be introduced easily, and it can be proposed that the existence of substructures will affect the roughness, creep, depinning and flow of DW 
lines in some manner, which may depend on the substructure density, supplying a novel way to modulate statics and dynamics of DW line.

This section is organized as following. In Sec. 3.2.2, we show some basic features of the phase field modeling we employed and the key parameters of the micromagnetic simulations. In Sec. 3.2.3, the roughness exponents as well as roughness widths of DW lines with various substructure densities are studied, and in order to better understand the thermal effect, the roughness features of DW lines without substructures at different temperatures are also studied. In Sec. 3.2.4, the motion behaviors of DW lines with different substructures densities driven by magnetic field in the creep, depinning and flow regimes are investigated. Finally, all the key conclusions are summarized in Sec. 3.2.5.

\subsubsection{Micromagnetic Model}

The evolution of magnetization is described by the Landau-Lifshitz-Gilbert(LLG) equation including the thermal fluctuations realized by the random thermal field $\mathbf{H}_{\mathbf{t h}}$, $[51,146]$

$$
\frac{\partial \mathbf{m}}{\partial t}=-\gamma \mathbf{m} \times\left(\mathbf{H}_{\mathrm{eff}}+\mathbf{H}_{\mathrm{th}}\right)+\alpha \mathbf{m} \times \frac{\partial \mathbf{m}}{\partial t},
$$

where $\mathbf{m}$ is a unit vector of magnetization, $\gamma$ the gyromagnetic constant, $\alpha$ the Gilbert

damping constant, $\mathbf{H}_{\text {eff }}$ the effective magnetic field obeying $\mathbf{H}_{\text {eff }}=-\delta E /\left(M_{s} \delta \mathbf{m}\right)$, and $\mathbf{H}_{\text {th }}$ the Gaussian distributed random fluctuating thermal field satisfying [146]

$$
\begin{gathered}
\left\langle H_{t h, i}(\mathbf{r}, t)\right\rangle=0, \\
\left\langle H_{t h, i}(\mathbf{r}, t) H_{t h, j}\left(\mathbf{r}^{\prime}, t^{\prime}\right)\right\rangle=2 \sigma_{t h}^{2} \delta_{i j} \delta\left(\mathbf{r}-\mathbf{r}^{\prime}\right) \delta\left(t-t^{\prime}\right),
\end{gathered}
$$


where $\sigma_{\text {th }}^{2}$ is temperature dependent of strength of the thermal field given by

$$
\sigma_{t h}^{2}=\frac{\alpha k_{B} T}{\gamma \mu_{0} M_{s} l^{3}}
$$

where $k_{B}$ is the Boltzmann constant, $T$ is the temperature, and $l$ the simulation grid size.

The parameters used to describe the magnetic system in our simulations are from the experimental results for a DW in $0.6 \mathrm{~nm}$ thick ultrathin $\mathrm{Pt} / \mathrm{Co} / \mathrm{Pt}$ film with perpendicular anisotropy [134]: saturation magnetization $M_{s}=1130 \mathrm{emu} / \mathrm{cm}^{3}$, uniaxial perpendicular magnetocrystalline anisotropy constant $K_{u}=1.25 \times 10^{7} \mathrm{erg} / \mathrm{cm}^{3}$, exchange constant $A=1.6 \times 10^{-6} \mathrm{erg} / \mathrm{cm}$, and Gilbert damping constant $\alpha=0.3$. The statistical defects may originate from many sources, for example, the surface roughness [151], edge roughness [51, 154] and magnetocrystalline anisotropy fluctuations [155] were all used as the pinning sources. In this section, the defects are realized by introducing a fluctuation of magnetocrystalline anisotropy obeying Gaussian distribution with an amplitude of $\delta K / K_{u} \approx 0.10$, since that type of defects is reported to be the main pinning source in ultrathin $\mathrm{Pt} / \mathrm{Co} / \mathrm{Pt}$ film with perpendicular anisotropy. [147]

For statics simulations, a DW line with length of $2.592 \mu \mathrm{m}$ with one grid size of $3.0 \mathrm{~nm}$ is used, and a serious of substructure densities are considered, i.e., $N=0,2,4,6,8$, 12, 18, 24, 36 and 48 per $2.592 \mu \mathrm{m}$. For dynamics simulations, only three kind of substructures densities are considered, $N=0,2$ and 4 per $0.288 \mu \mathrm{m}$, which is just $N=0,18$ and 36 per $2.592 \mu \mathrm{m}$ for statics cases, and in the reminder part of this paper we will only use $N=18$ or 36 instead of $N=2$ or 4 to avoid confusion. For those three cases, since long range interaction between domains exists, large system size of $5.40 \mu \mathrm{m} \times 0.288 \mu \mathrm{m}$ with 
one grid size of $3.0 \mathrm{~nm}$ is used to reduce the stray field effect acting on the DW. In our simulations, actually, the distance between two neighbor domain walls is always larger than $2.2 \mu \mathrm{m}$, resulting in ignorable stray field acting on the DW. Fig. 3.4 shows the motion behavior of DW line with $N=18$ (i.e., $N=2 / 0.288 \mu \mathrm{m}$ ) substructures driven by the magnetic field of $0.0039 M_{s}$. It is noted that the thermal field as well as the statistical defects make the DW line roughened and that the long range interaction between DW lines is ignorable in this short moving range.

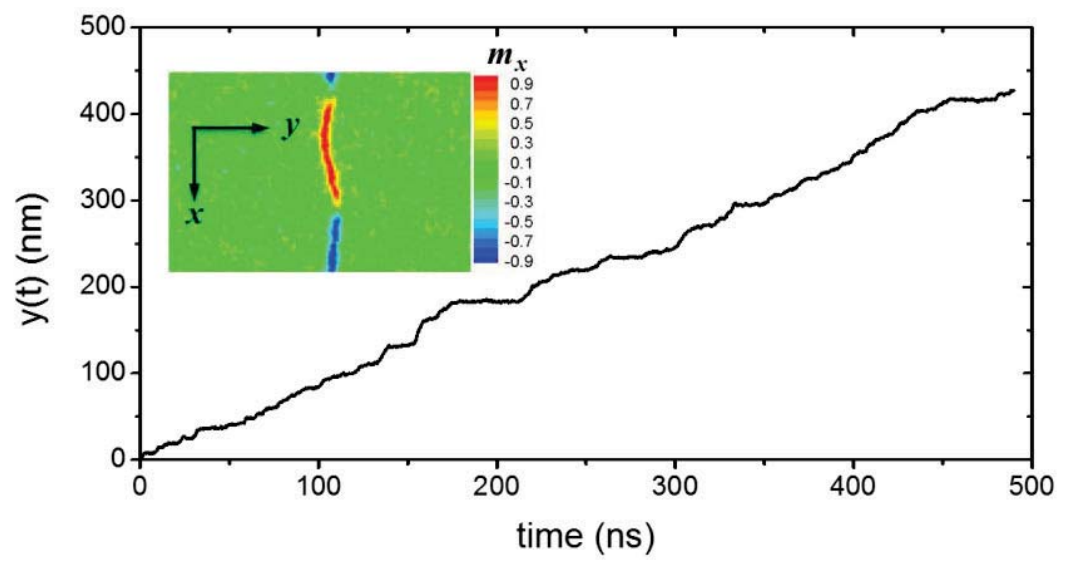

Figure 3.4 Average position of DW line with substructure density $N=18$ per $2.592 \mu \mathrm{m}$ vs. time under magnetic field $0.0039 M_{s}$. The inset is the magnetization structure of moving DW line, and the color contour represents the magnetization component in $\mathrm{X}$ direction, $m_{x}$.

\subsubsection{Statics of Domain Wall Lines with Substructures}

The magnetization structure of a substructure within Bloch type DW line is schematically shown in Fig. A1. In fact, if the DW line is analogy to a very narrow planer 'nanowire', the substructure may be analogy to the Neel type transverse head-to-head(hh) or tail-to-tail (tt) 'DW' in the 'nanowire', and the charged 'DW' will result in higher magnetostatic energy in addition to the extra exchange energy caused by the extra 
varying magnetization structure. Along X-direction, the magnetizations within DW can rotate clockwise (cw) or counterclockwise (ccw) across the substructures, i.e., with different chiralities, which will classify those substructures into four types, cw-hh, cw-tt, ccw-hh and ccw-tt. In the DW line, once the cw and ccw substructures meet together, they will be annihilated and then the substructure density will be decreased. In order to avoid such annihilations and keep the substructure density unchanged during the static and dynamical processes, only ccw type substructures are allowed in our cases, i.e., only the ccw-hh and ccw-tt type substructures are introduced within DW lines.

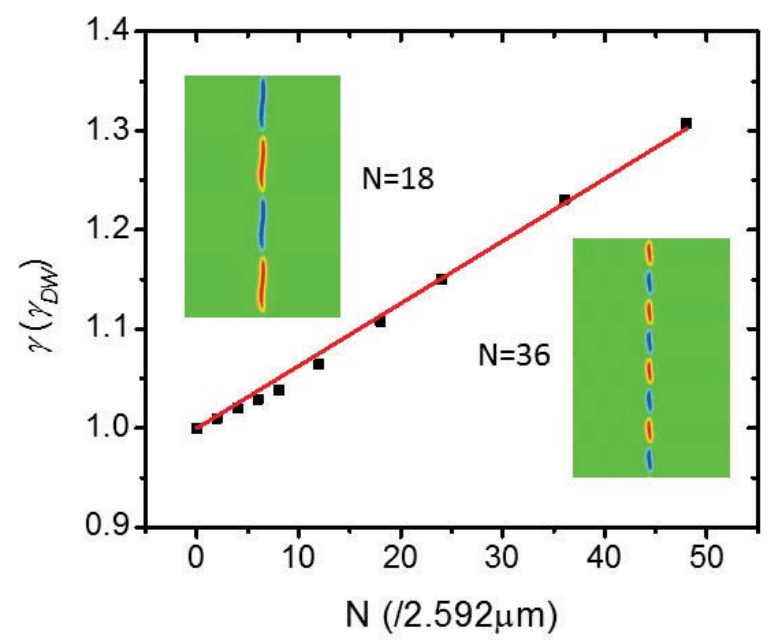

Figure 3.5 Linear fitting of the total energy density $\gamma$ (divided by $\gamma_{D W}$, the pure DW energy density) of DW with substructures vs. the substructure density $N$. Black points are simulated data and red line is the fitting curve. Two insets are the ideal magnetization structure of DW lines segments with substructure density $N=18$ and 36 .

Based on our simulation results, the substructures in ideal magnetic film, i.e., without any pinning sites or thermal fluctuations, have a length of $28.5 \mathrm{~nm}$ along $\mathrm{X}$ - 
direction and a width along Y-direction of $\sim 6 \mathrm{~nm}$ which is almost the same with DW line width. Our calculated result according to Eq. (A4), actually, gives a somewhat smaller value, $\sim 22.5 \mathrm{~nm}$. Since substructures possess more energy, the total DW energy density $\gamma$ depends on substructures density $N$. Fig. 3.5 shows an almost linear dependent of DW energy density $\gamma$ in unit of $\gamma_{D W}=4 \sqrt{A K}$, the ideal pure DW energy density, on the substructures density $N$ per $2.592 \mu \mathrm{m}$, according to our simulation results. Fitting the simulated data in Fig. 3.5 linearly, the relationship between $\gamma$ and $N$ can be obtained,

$$
\gamma / \gamma_{D W}=1+0.0063 N
$$

demonstrating a coefficient $\eta=1.57$, a little higher than the calculated value in Appendix A. Actually, by looking at the data in Fig. 3.5 carefully, it can be noted that the slop for lower substructure density range is a little bit lower while for higher substructure density the slop is higher, which should be due to the densely packing effect of high density substructures in a DW line. Both the calculated substructure length and energy density indicate that the simple approximation in Appendix A underestimates the complicated magnetostatic effect of substructures, i.e., the charged substructures possess larger length and energy than approximated. Since the elasticity of DW line $\varepsilon_{e l}$ is linearly dependent of DW energy [135], DW line with substructures will have higher elasticity according to $\varepsilon_{e l} \propto \eta \gamma_{D W}$. Usually, higher elasticity can lead to DW line deroughening, and hence substructures can deroughen DW line in somewhat extent as discussed in the following, but it is obvious that more factors besides of elasticity should be considered for the roughness phenomenon because of the additional motion freedom degree via substructures. 

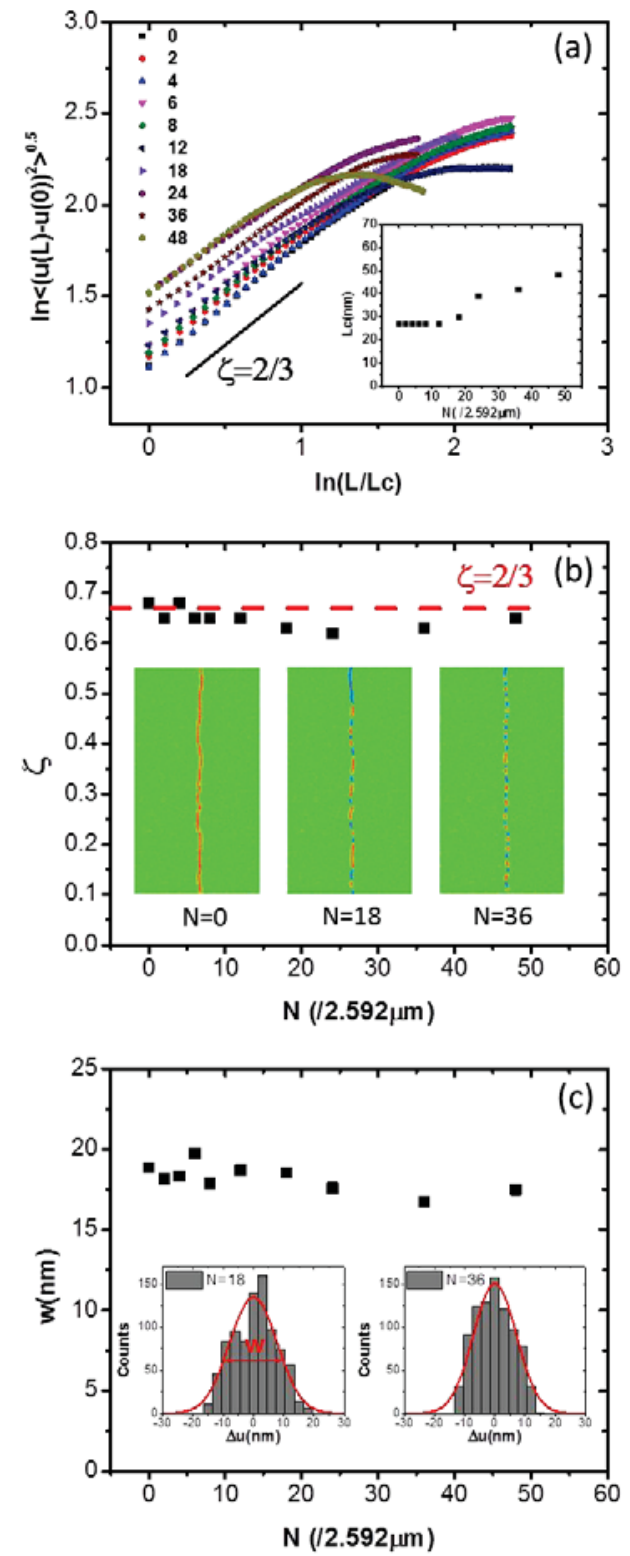

Figure 3.6 (a) Transverse displacement correlation functions describing rough DW lines with different substructure density $N=0,2,4,6,8,12,18,24,36$ and 48 per $2.592 \mu$ mat $300 \mathrm{~K}$ calculated from Eq. (3.2.6). Inset is the corresponding characteristic collective pinning length $L_{c}$. (b) Roughness exponents $\zeta$ for DW lines with those different substructure densities. Insets are the simulated DW lines with substructure density $N=0,18$ and 36 at $300 \mathrm{~K}$. (c) Roughness widths w obtained by Gaussian peak fitting for the Gaussian distributed lateral DW wandering $\Delta u$ of DW lines with different substructure densities. Insets are two Gaussian peaks fitting for cases $N=18$ and 36 . 
Starting with DW lines with different substructure densities at zero temperature, i.e., DW lines stabilized by the potential fluctuated pinning sites, the roughness of DW lines at 300K after a relative long time evolution $\sim 300 \mathrm{~ns}$ is studied. The insets of Fig. 3.6 (b) show the DW lines at $300 \mathrm{~K}$ with different substructure densities, $N=0,18$ and 36 per $2.592 \mu \mathrm{m}$. For roughness, the roughness exponents and the roughness widths, both of which are dependent of substructure density, are taken into account in this section. The roughness exponent $\zeta$ can be obtained from the transverse displacement correlation function [128]:

$$
\left\langle[u(x+L)-u(x)]^{2}\right\rangle \propto u_{0}^{2}\left(\frac{L}{L_{c}}\right)^{2 \zeta}
$$

where $u_{0}$ is the transverse scaling parameter and $L_{c}$ is the characteristic collective pinning length or Larkin length. Fig. 3.6 (a) shows the correlation function described in Eq.(3.2.6) for DW lines with a set of substructure densities from $N=0$ to 48 . The characteristic pinning lengths are shown in the inset of Fig. 3.6 (a). The roughness exponent $\zeta$ can be obtained by linearly fitting the initial linear part of correlation function in Fig. 3.6 (a), i.e., at short length scales (Regime I) the lines have reached equilibrium within the random pinning potential, and beyond that regime there exists a plateau which is growing with time [156] (Regime II). Length scales in Regime I can also grow with time. The fitted exponents $\zeta$ are shown in Fig. 3.6 (b) and all of them are very close to the wellknown value $\zeta=2 / 3$, but for $N>12$, those values are smaller than $2 / 3$ with some of them even as low as $\sim 0.6$, indicating that substructures can more or less reduce roughness exponent in a certain manner, which will be discussed later. The roughness width can be described through the Gaussian distributed lateral DW wandering $\Delta u$, and the width of 
such a Gaussian peak, w, is treated as the DW line roughness width [136]. The Gaussian peak fitting of lateral wanderings for $N=18$ and 36 are shown by the insets of Fig. 3.6 (c). All those roughness widths for DW lines with various substructure densities are shown in Fig. 3.6 (c). No evident roughening or deroughening effect can be seen from Fig. 3.6 (c), but a slight deroughening effect for DW line with high substructure density $N>18$ seems to be existed, which is also indicated by Fig. 3.6 (a), the reduced plateau values for increased substructure densities. Such a slight deroughening effect may be contributed by the substructure density dependent of elasticity $\varepsilon_{e l}$ discussed above, but the increased elasticity is still unable to explain the reduced roughness exponents in Fig. 3.6 (b).

Based on our calculated result in Eq. (B8), the thermal fluctuations at substructures are drastically enhanced up to $\sim 12$ times of that at DWs with $\alpha=0.3$, i.e., the effective temperature at substructures can be as large as $~ 12$ times of applied temperature $T=300 \mathrm{~K}$. Confined in the thin film, DW line can move in one dimension while substructures can move two dimensionally. For the transverse motion, i.e., in Y-direction, DW line has only one dimensional driving force according to Eq. (B7), while substructures have two dimensional driving forces according to Eq. (B6), and thus the fluctuated thermal field will attribute more to the substructures, which is why the thermal effect is enhanced at substructures. Actually, since our calculations in Appendix B ignored the interactions between the substructures and pure DW segments as well as between neighboring substructures, such a huge value sets the up limit of effective temperature, i.e., the real effective temperature should be smaller or perhaps even much smaller than the up limit value, $\sim 12 \mathrm{~T}$, due to such interactions. To look into the 
temperature effect on DW lines closely, pure DW lines at different temperatures are studied as following.

Fig. 3.7 shows roughness exponents as well as roughness widths for pure DW lines at different temperatures increasing from $T=0$ to $1200 \mathrm{~K}$ after $\sim 100 \mathrm{~ns}$ evolution starting from the initial state of zero temperature. The evolved rough DW lines at $T=0$, 300 and $1200 \mathrm{~K}$ are shown by the insets of Fig. 3.7 (b). Fitting linearly at Regime I according to Eq. (3.2.6) for all those rough DW lines at various temperatures as shown in Fig. 3.7 (a), the roughness exponents are obtained and shown in Fig. 3.7 (b). It is noted that for $T \leq 750 \mathrm{~K}$, the roughness exponents are in excellent agreement with the equilibrium value $2 / 3$, while for $T>750 \mathrm{~K}$, those values approach the thermal dominated roughness exponent value $\zeta_{T}=1 / 2$ [128]. For example, the roughness exponent is 0.61 at $T=900 \mathrm{~K}$ and 0.54 at $T=1200 \mathrm{~K}$, for which the thermal fluctuations are huge enough to influence the exponent value. Evidently, for $T>1200 \mathrm{~K}$, the roughness exponent value will be around $1 / 2$ since the thermal effect will be dominated for such high temperatures, while for temperatures between $750 \mathrm{~K}$ and $1200 \mathrm{~K}$, the roughness exponent will be between those two characteristic values, i.e., $\zeta \in(1 / 2,2 / 3)$, which is consistent with other numerical simulations [152]. It is also noted that at low temperatures, even as low as $0 \mathrm{~K}$, the roughness exponent persists $2 / 3$ in Fig. 3.7 (b), which is inconsistent with Ref. [152] in which a much higher roughness exponent has been found for the low temperature regime. The insets of Fig. 3.7 (a) show that the Larkin lengths are increased with increasing temperatures, in agreement with the theoretical expectation that strong thermal fluctuations can drastically increase the Larkin length of one dimensional elastic string in two dimensional random media [139]. Strong thermal fluctuations can not only increase 
the Larkin length, but also increase the roughness width. Fig. 3.7 (c) shows the temperature dependent of roughness width obtained from the Gaussian distributed lateral wandering $\Delta u$. It is noted that the roughness widths are increased with temperatures, i.e., DW line roughening can be induced by thermal fluctuations. A simple scaling $u \propto T^{0.5}$ has been reported for elastic string roughening induced only by thermal effect [139], and our results show that for higher temperatures at which the thermal effect is stronger, those roughness width values are able to be better fitted by $\mathrm{w} \propto T^{0.5}$, consistent with the simple scaling law.

As discussed above, thermal fluctuations can increase both the Larkin length and the roughness width, and strong thermal fluctuations $(T>750 \mathrm{~K})$ can reduce the roughness exponent to as low as $\zeta_{T}=1 / 2$ from $\zeta=2 / 3$. Since thermal fluctuations can be enhanced at substructures, thermal effect should be taken into account for the statics study of DW line with substructures. At $T=300 \mathrm{~K}$, once the thermally enhanced temperature at substructures exceed 2.5T, much lower than the up limit value $\sim 12 T$, the roughness exponent at substructure part will begin decreased, which causes the roughness exponent of the whole DW line lower than the equilibrium value $\zeta=2 / 3$. The higher the substructure density, the lower the roughness exponent value. For DW line with densely packed substructures, for example, substructures occupy one half of whole DW line length, which corresponds to $N \sim 45$, the exponent value may be the average of $\zeta_{T}=1 / 2$ and $\zeta=2 / 3$, i.e., $\sim 0.58$, in a simple approximation. Consequently, for $N<45$, the roughness exponent will be higher than 0.58. However, for densely packed substructures, their motion within DW line is partially restricted as shown by the insets of Fig. 3.6 (b) for cases $N=18$ and 36, i.e., one dimension freedom degree in X-direction is limited, and hence the thermal enhancement 
is relatively weakened and the roughness exponent is relatively increased. Thinking of a DW line with most densely packed substructures, i.e., no pure DW segment exists, those substructure can't move within DW line, and the DW line will be rather a pure DW line with higher elasticity, but still possesses a roughness exponent $\zeta=2 / 3$. In short, based on our analysis, for both low and high substructure density, the roughness exponent will be relatively high, i.e., slightly lower than $\zeta=2 / 3$, while for medium, the value will be relatively low, but not lower than $\sim 0.58$ according to the simple approximation, all of which are consistent with our simulated results shown in Fig. 3.6 (b). Besides, enhanced thermal effect can increase Larkin length, as shown by the inset of Fig. 3.6 (a), and the increased elasticity can also attribute to the Larkin length increment [139]. Although the thermal enhancement can increase roughness width of a DW line, it seems not a dominant effect, or at least not as competitively as elasticity which can decrease roughness widths, according to the roughness widths shown in Fig. 3.6 (c). As discussed above, thermal enhancement can be weakened for many reasons, and besides of them, the low mobility of substructures according to Eq. (B4) can also limit the transverse displacement of a DW line and weaken the thermal enhancement accordingly. 

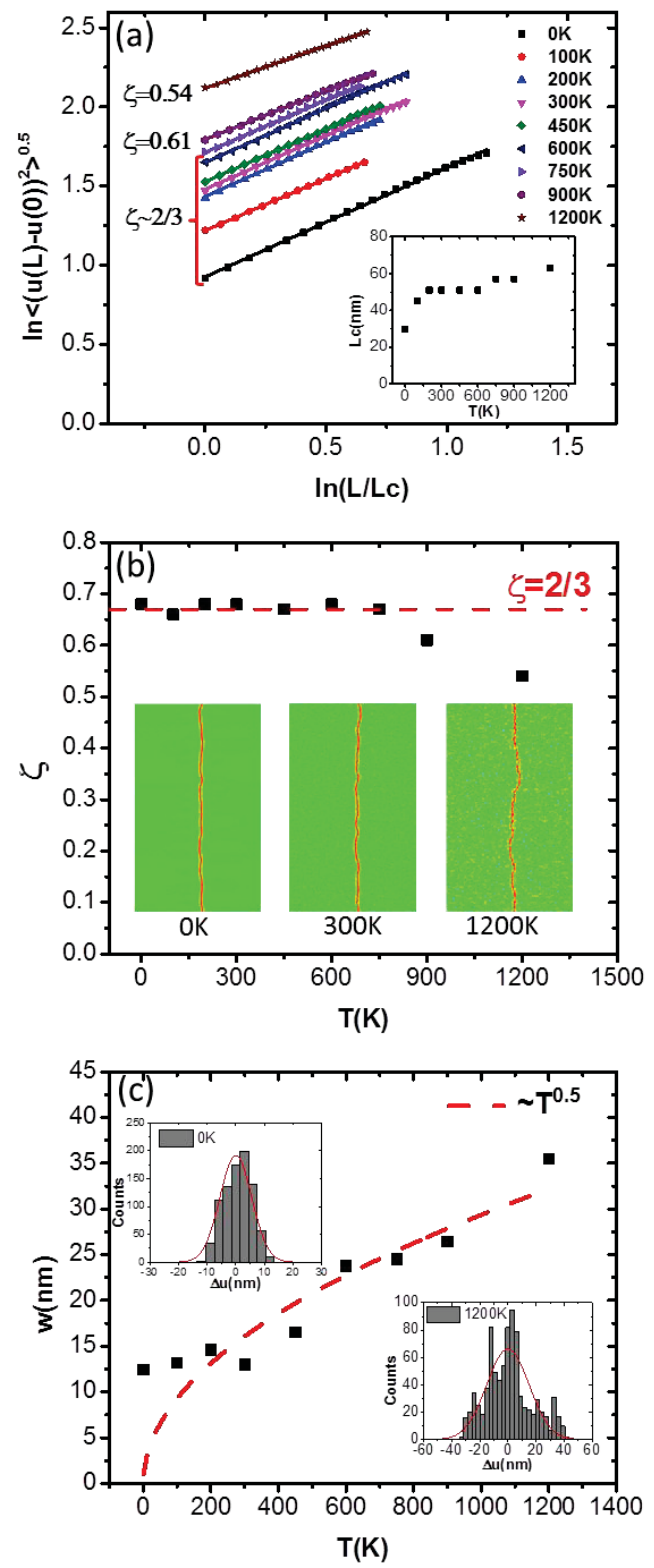

Figure 3.7 (a) Linear fitting for Regime I of transverse displacement correlation functions describing rough DW lines without substructures at different temperatures $T=0,100,200,300$, 450, 600, 750, 900 and $1200 \mathrm{~K}$ calculated from Eq. (3.2.6). Inset is the corresponding characteristic collective pinning length $L_{c}$. (b) Roughness exponents $\zeta$ for DW lines at those different temperatures. Insets are the simulated DW lines at $T=0,300$ and $1200 \mathrm{~K}$. (c) Roughness widths w obtained by Gaussian peak fitting for the Gaussian distributed lateral DW wandering $\Delta u$ of DW lines at different temperatures, fitted by a function $\propto T^{0.5}$. Insets are two Gaussian peaks fitting for cases $T=0$ and $1200 \mathrm{~K}$. 


\subsubsection{Dynamics of Domain Wall Lines with Substructures}

\subsubsection{Flow Regime}

DW lines begin to move when external magnetic field is applied to the rough DW line with three different substructure densities, $N=0,18$ and 36 per $2.592 \mu \mathrm{m}$. Since the velocity is not uniform due to the thermal fluctuations and pinning sites as shown in Fig. 3.4, the average velocity is measured. Fig. 3.8 (a) shows the velocity-field relationship in the whole regime with magnetic field increasing up to $0.2 M_{s}(\sim 2840$ Oe $)$. There are two types of flow regimes separated by the threshold $H_{W}$, the Walker field, below which the steady linear flow regime exists while above the precessional linear flow regime exists, as shown in Fig. 3.8 (a) and (b). The Walker field values for the three cases are listed in Tab. 3.2. It is noted that the existence of substructures increases the Walker field, for example, the Walker field is $0.015 M_{s}$ for pure DW while $0.018 M_{s}$ and $0.026 \mathrm{Ms}$ for DW with substructure density $N=18$ and 36 , respectively. The spin torque generated by the applied magnetic field is stored at DW during the moving process, which can be described by the angle $\varphi$ mentioned in Appendix A. Increasing magnetic field will increase the spin torque, once the spin torque is too big or $\varphi$ is beyond a critical value, DW will not be stable anymore and that critical magnetic field is called Walker field. The substructures existed at DW will help reduce the generated spin torque by means of sliding within the DW, and almost all of the generated spin torque can be drained out in time by substructures in the ideal case that no pinning sites or thermal fluctuations are included, resulting in a very large Walker field. In real cases, however, only partial spin torque near the substructures can be effectively eliminated by the motion of substructures due to the obstruct of pinning sites, leading to larger but not so large Walker field. Higher 
substructure density means higher spin torque elimination effect and hence larger Walker field.
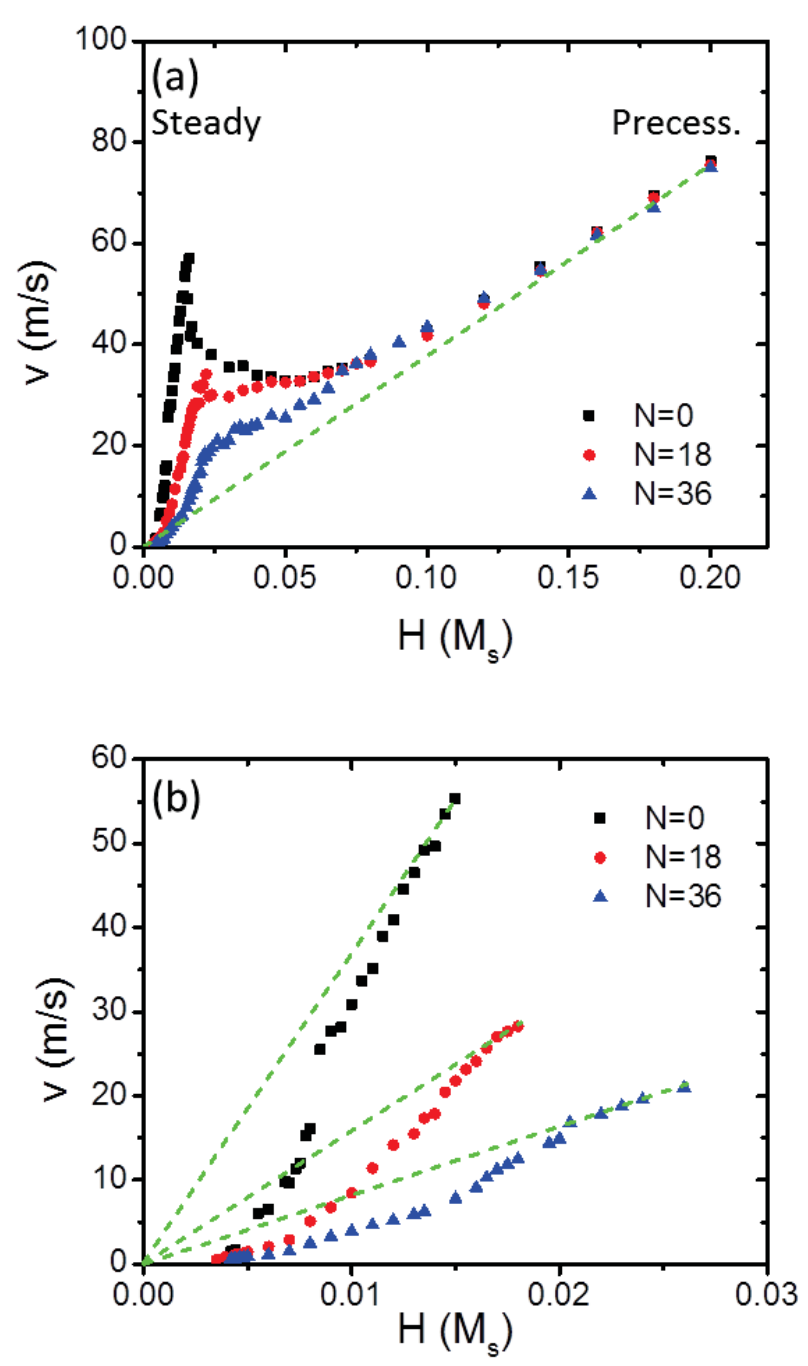

Figure 3.8 (a) Simulated velocity-field relationship of rough DW lines with substructure densities $N=0,18$ and 36 at 300K. Dashed green line fits the precessional linear flow regime. (b) Simulated velocity-field relationship under Walker field for cases $N=0,18$ and 36 at $300 \mathrm{~K}$. Dashed green lines fit the steady linear flow regime. 
Once the magnetic field is beyond the Walker field, the DW become unstable and pairs of substructures may be generated in form of hh and tt types with opposite chirality which can be annihilated by each other easily. When more substructure pairs are generated, the difference of velocity-field relationship between DW line with and without substructures will be smeared into the uniform one, which can be shown by Fig. 3.8 (a) that the velocity-field relationship for the case of $N=18$ and $N=36$ begins to merge at $\sim 0.05 M_{s}$ and $\sim 0.07 M_{s}$, respectively. Therefore, all the cases for DW line with or without substructures will share the same motion mobility at the precessional linear flow regime well above the Walker field, $m_{\text {pre }}=0.027 \mathrm{~m} / \mathrm{s} \cdot \mathrm{Oe}$, much lower than that at steady linear flow regime.

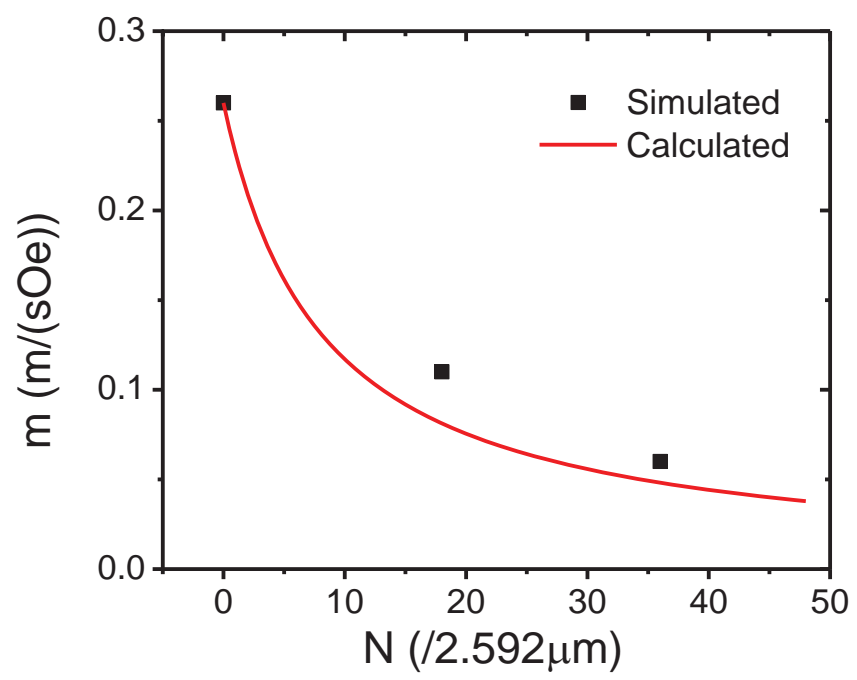

Figure 3.9 Calculated (red curve according to Eq. (3.2.7)) and simulated (black points) mobility $m$ of DW line with different substructure densities.

For the steady linear flow regime slightly below Walker field, the existence of substructures can effectively decrease the DW line mobility or velocity by performing a 
sliding motion along the DW line. For the ideal case discussed in Appendix A, the mobility for DW line with substructures is about only1/12 of that for pure DW line, which is almost independent of substructure density. For cases considering pinning sites and thermal fluctuations, since spin torque generated at DW can't be eliminated completely as discussed above, the mobility magnitude of DW lines with substructures will be between that of ideal pure DW line and that of DW line with substructures, dependent of substructure density. All the three values of mobility for cases of $N=0,18$ and 36 are listed in Tab. 3.2 and shown in Fig. 3.9 as well, demonstrating that DW mobility can be reduced by increasing the substructure density. In an approximation, the DW mobility can be calculated by

$$
\frac{1}{m_{c a l}}=\frac{w_{s u b}}{m_{s u b}}+\frac{1-w_{s u b}}{m_{D W}},
$$

where $m_{\text {sub }}=\frac{\alpha \gamma \lambda}{1+\alpha^{2}}$ is the ideal substructure mobility, $m_{D W}=\frac{\gamma \lambda}{\alpha}$ the ideal DW mobility, and $w_{\text {sub }}$ the substructure fraction which might be expressed by means of length, i.e., $w_{\text {sub }} \approx \frac{N l_{\text {sub }}}{L}$, where $l_{\text {sub }} \approx 28.5 \mathrm{~nm}$ is the substructure length. The calculated result is plot in Fig. 3.9, which is scaled with respect to the value of case $N=0$. Fig. 3.9 shows that the calculated result agrees well with the simulated result, although our approximation slightly underestimates the DW mobility. It is noted that DW mobility drops relatively drastically for low substructure density while slowly for high substructure density.

\subsubsection{Depinning Regime}


In the depinning regime, it is assumed that the velocity-field relationship obeys the power law: [138]

$$
v \propto\left(H / H_{c}-1\right)^{\beta},
$$

where the depinning exponent $\beta=1 / 3$ is expected by numerical simulations[144, 145] and has been further supported experimentally most recently [132]. A series of different test values of $H_{c}$ with interval of $0.0005 M s$ are used to make linear fittings according to Eq. (3.2.8), and the value corresponding to the exponent $\sim 1 / 3$ is determined as the critical depinning field $H_{c}$. The linear fitting results for cases $N=0,18$ and 36 with exponent $\beta \sim 1 / 3$ are shown in Fig. 3.10 (a), and the relevant determined depinning field values are listed in Tab. 3.2. Those depinning field values demonstrate that introducing substructures will increase the DW line pinning force and hence lead to a high depinning field. According to Eq. (B2) and (B3), the pinning force for substructure, $f_{\text {sub }} \sim f_{1}+f_{2} / \alpha$, is higher than that for pure DW, $f_{D W} \sim f_{1}$, and the additional force $f_{2}$ is mostly corresponding to the sliding motion of substructures within DW line. In other words, in order to depin the DW line, the driving field need not only overcome the barrier preventing forward motion perpendicular to DW line, but also overcome the barrier preventing slide motion along DW line due to the existence of substructures, leading to higher pinning force which is also dependent of substructure density. It can be assumed further that the total pinning force $f$ and the depinning field $H_{c}$ increases linearly with respect to the substructure density $N$. Based on those three calculated depinning field values, we have

$$
H_{c} \approx(0.0095+0.00017 N) M_{s} .
$$


Although it is hard to determine the exact magnitude of the pinning force $f_{1}$ and $f_{2}$, the ratio between them can be estimated according to Eq. (3.2.9), $f_{2} / f_{1} \sim 0.8$, i.e., both of the forces are in the same order but the additional term $f_{2} / \alpha$ will be larger due to the small $\alpha$.
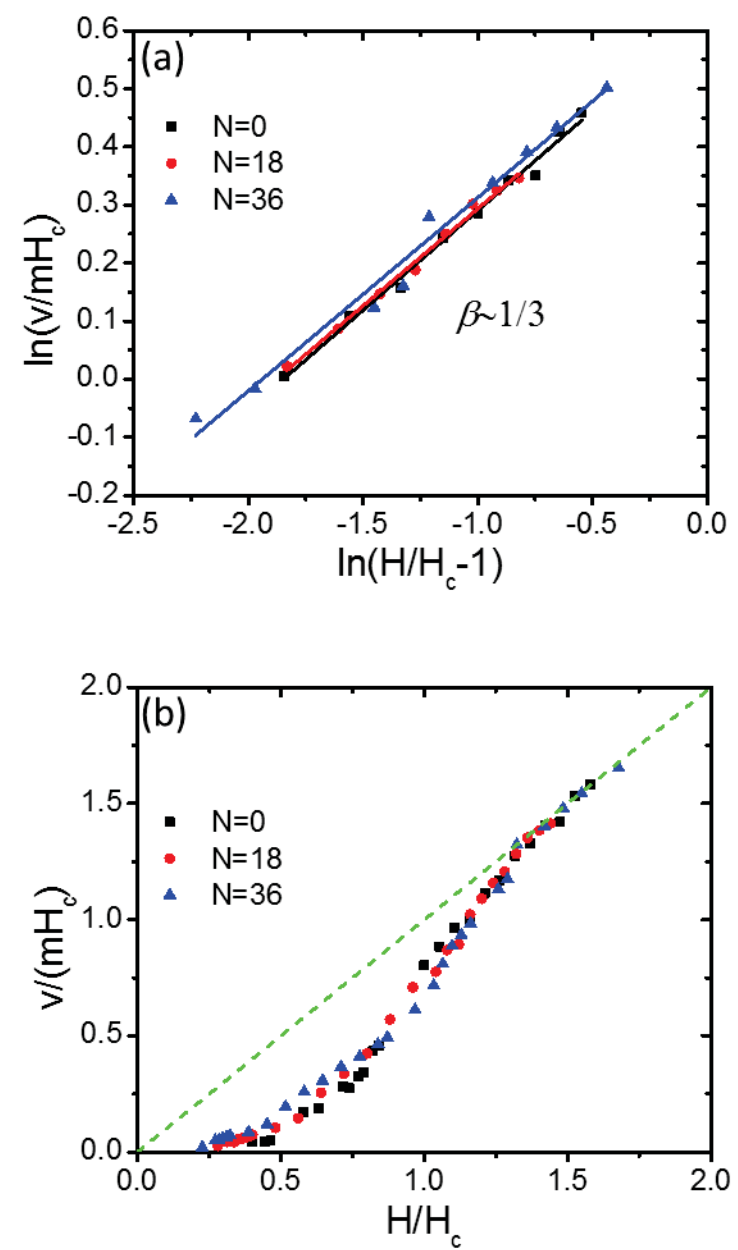

Figure 3.10 (a) Linear fittings for velocity-field relationship of cases $N=0,18$ and 36 at the depinning regime with exponent $\beta \sim 1 / 3$ according to Eq. (3.2.8). (b) Normalized velocity-field relationship under Walker fiend for cases $N=0,18$ and 36, i.e., velocity is normalized by $m H_{c}$ and magnetic field is normalized by $H_{c}$. 
Since both the mobility at the steady linear flow regime and depinning field at the depinning regime are determined, the velocity-field relationship can be normalized, i.e., velocity is normalized by $m H_{c}$ and magnetic field is normalized by $H_{c}$, as shown by Fig. 3.10 (b). Such normalization actually merged the data in the flow regime, leaving data in other regimes still unmerged. In the creep regime particularly, for $H / H_{c}$ under $\sim 0.6$, the DW line with higher substructure density shows higher velocity, indicating smaller pinning barrier energy [132], which will be discussed in the following.

\subsubsection{Creep Regime}

There are two sub-regimes in the creep regime, one is for $H<<H_{c}$ (well below the depinning field) and the other is for $H \leq H_{c}$ (slightly below the depinning field), which have been studied in Sec. 3.1. Both regimes can be described by the Arrhenius law [128]

$$
v=v_{0} \exp (-U / k T),
$$

where $U=U_{c 1}\left(H_{c} / H\right)^{\mu}$ with a universal constant exponent $\mu=1 / 4$ for $H<<H_{c}$, and $U=U_{c 2}\left(1-H / H_{c}\right)^{\alpha}$ with the exponent $\alpha=5 / 4$ for $H \leq H_{c}$ obtained based on the washboard potential model [143]. Fitting the velocity-field relationship according to Eq. (3.2.10) with the above exponent for $H<<H_{c}$, the pinning barrier energy $U_{c 1}$ can be obtained. Fig. 3.11 (a) shows the linear fitting for the velocity-field relationship according to Eq. (3.2.10) at the regime $H \ll H_{c}$, and the three pinning energies divided by $k T$ for cases $N=0,18$ and 36 are listed in Tab. 3.2. 

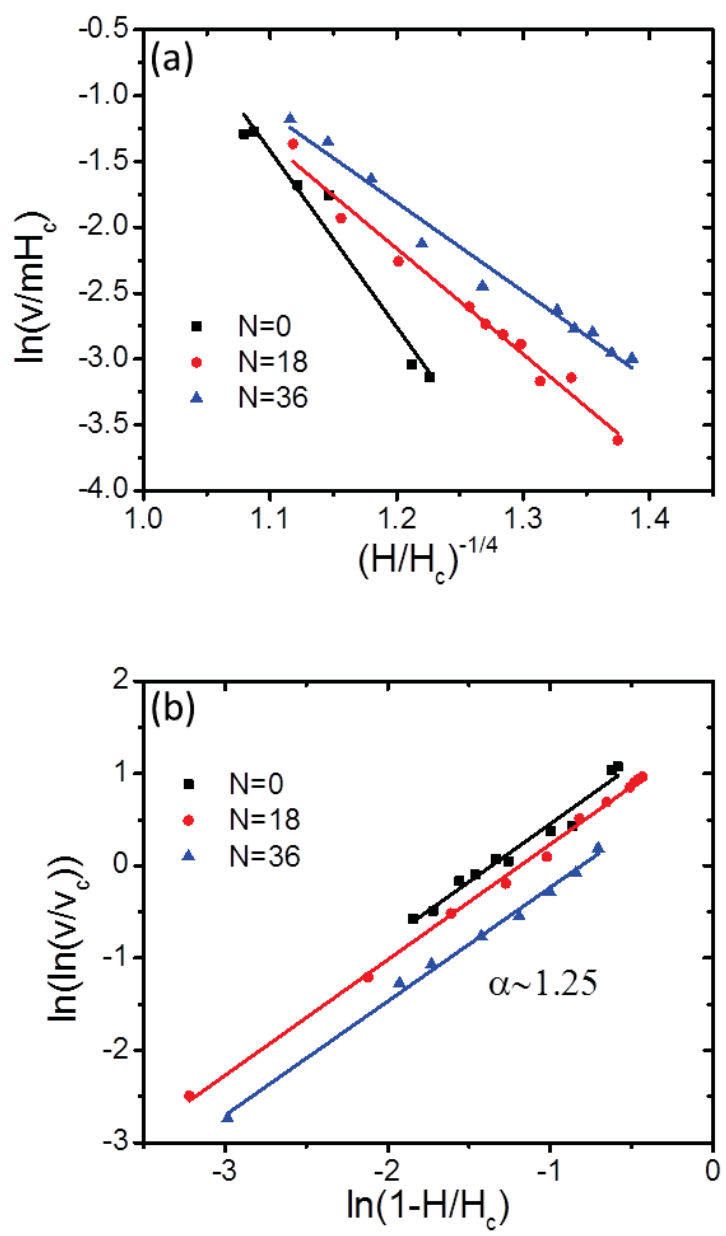

Figure 3.11 Linear fittings for velocity-field relationship of cases $N=0,18$ and 36 at the creep regime with $H<<H_{c}$ (a) and $H \leq H_{c}$ (b) according to Eq. (3.2.10) with two types of pinning energies.

The three decreasing values of $U_{c 1} / k T$ which are also shown in Fig. 3.12, actually, explain the increasing velocities for increased substructure densities with $H / H_{c}$ under $\sim 0.6$ as shown in Fig. 3.10 (b), since either reducing the pinning barrier or increasing the temperature can speed up the DW line creep under small field. However, 
the reason why the value of $U_{c 1} / k T$ is decreased by increasing substructure density still needs to be analyzed. For pinning energy $U_{c 1}$, we have $U_{c 1}(N) \propto \sqrt{\varepsilon_{e l}(N) H_{c}(N)}$, since both the string elasticity $\varepsilon_{e l}$ and depinning field $H_{c}$ are linearly dependent of substructure density $N$ according to Eq. (3.2.5) and (3.2.9), respectively. For temperature $T$, since the existence of substructures can enhance thermal fluctuations according to Eq. (B8) and hence drastically increase the temperature, the temperature $T$ is also dependent of substructure density. At the substructure, the maximum enhanced temperature is $T_{\text {sub }}=\left(1+\alpha^{2}\right) / \alpha^{2} T \approx 12 T$, but there is no obvious principle to tell how it contributes into the effective temperature of the whole DW line. In a simple approximation, we assume that the temperature is evenly distributed within the DW line, and then we have

$$
T_{\text {eff }}=w_{\text {sub }} T_{\text {sub }}+\left(1-w_{\text {sub }}\right) T
$$

where $w_{\text {sub }}$ is still the length fraction of substructures and proportional to substructure density. However, since the slide motion of substructure can be prevented by neighboring substructures as shown by the insets of Fig. 3.6 (b), such a drastic temperature enhancement can be reduced, which is more evident for DW line with higher substructure density, and hence the approximated calculation result according to Eq. (3.2.11) is actually the upper limit of the effective temperature. Therefore, the lower limit of $U_{c 1} / k T_{\text {eff }}$ based on the above approximation can be calculated by scaled by the value of case $N=0$, which is shown in Fig. 3.12. It is noted that the simulated values for cases $N=18$ and 36 are higher than the calculated lower limit as expected. 


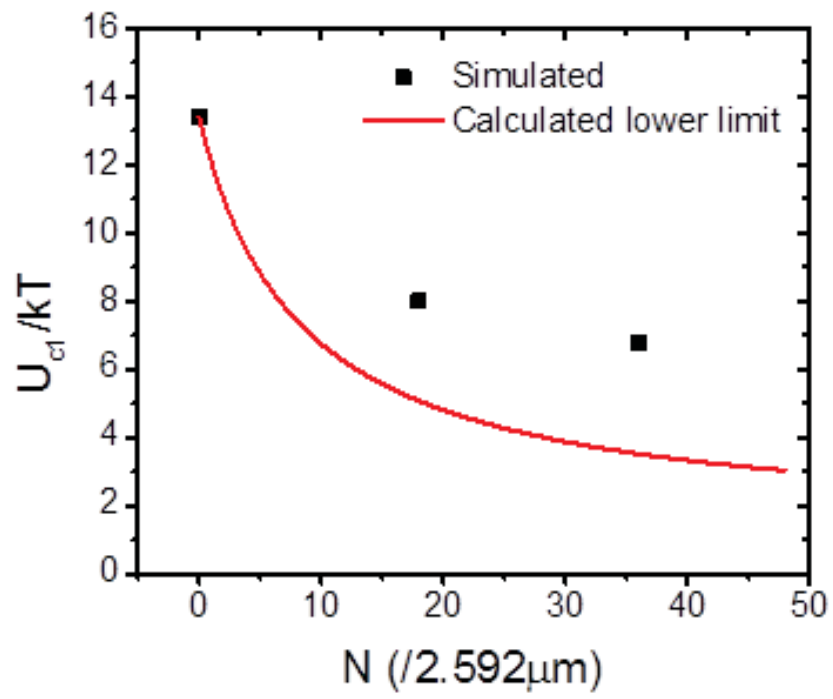

Figure 3.12 Calculated lower limit (red curve) and simulated (black points) values of $U_{c 1} / k T_{e f f}$ for DW line with different substructure densities at the creep regime with $H<<H_{c}$.

In the creep regime with $H \leq H_{c}$, since the depinning field is determined yet, both the exponent $\alpha$ and pinning energy $U_{c 2}$ can be obtained by fitting from our simulated results, just as what we did in Sec. 3.1. Fig. 3.11 (b) shows the fitting result for the field slightly below the depinning field, resulting in the exponent $\alpha$ which can be found in Tab. 3.2 with all the values around 1.25, supplying a further support for the exponent value $\alpha=4 / 5$ calculated based on the washboard mode. All the three values of $U_{c 2} / k T$ are also listed in Tab. 3.2, which indicate a decreasing trend along with increased substructure density. The ratio for both pinning energies, $U_{c 2} / U_{c 1}$, are also calculated and the results are listed in Tab. 3.2. In good agreement with our previous work in Sec. 3.1, the pinning energy ratios are smaller than unity and around 0.5 , demonstrating close disorder lengths in the two sub-regimes. 
Table 3.2 Substructure density, $N$, Walker field, $H_{W}$, DW line mobility at steady linear flow regime, $m$, depinning field, $H_{c}$, normalization parameter, $m H_{c}, U_{c 1} / k T$ at creep regime with $H<H_{c}, U_{c 2} / k T$ at creep regime with $H \leq H_{c}$, the ratio for the two pinning energies, $U_{c 2} / U_{c 1}$, and creep exponent $\alpha$ with $H \leq H_{c}$, obtained from the dynamics study of DW lines.

\begin{tabular}{lcccccccc}
\hline $\begin{array}{c}N \\
(/ 2.592 \mu \mathrm{m})\end{array}$ & $\begin{array}{c}H_{W} \\
\left(M_{s}\right)\end{array}$ & $\begin{array}{c}m \\
(\mathrm{~m} / \mathrm{s} \cdot \mathrm{Oe})\end{array}$ & $\begin{array}{c}H_{c} \\
\left(M_{s}\right)\end{array}$ & $\begin{array}{c}m H_{c} \\
(\mathrm{~m} / \mathrm{s})\end{array}$ & $U_{c 1} / k T$ & $U_{c 2} / k T$ & $U_{c 2} / U_{c 1}$ & $\alpha$ \\
\hline 0 & 0.015 & 0.26 & 0.0095 & 35 & 13.4 & 5.5 & 0.41 & 1.26 \\
18 & 0.018 & 0.11 & 0.0125 & 20 & 8.0 & 4.4 & 0.55 & 1.25 \\
36 & 0.026 & 0.06 & 0.0155 & 12.7 & 6.8 & 2.7 & 0.40 & 1.23 \\
\hline
\end{tabular}

\subsubsection{Conclusion}

To summarize, the statics and dynamics of DW line with substructures in ultrathin magnetic film at finite temperature are studied based on our micromagnetic simulations. For statics, the roughness widths and roughness exponents for DW line with different substructure densities are investigated. Mainly through increasing DW energy as well as the corresponding elasticity constant $\varepsilon_{e l}$, the existence of substructures can slightly reduce the roughness width and hence more or less deroughen the DW line. The roughness exponent can be also decreased by introducing substructures due to the thermal fluctuation enhancement at substructures. The thermal effect on the roughness width and roughness exponent for the pure DW line without substructures is also studied at various temperatures. Our simulated results show that DW can be roughened by thermal fluctuations, and the roughness exponent $\zeta$ will be reduced when the temperature is beyond a critical value and finally approach $\zeta_{T}=1 / 2$ at higher temperature, which explains 
the reduction of roughness exponent for DW lines with substructures that cause the thermal enhancement.

For dynamics, the motion behaviors of DW line with different substructures densities under external magnetic field at $300 \mathrm{~K}$ in the creep, depinning and flow regimes are studied. At the flow regime, two linear flow regimes exist, the steady linear flow regime slightly below the Walker field and the precessional linear flow regime well above the Walker field. The Walker field can be increased by introducing substructures which will partially drain the field induced spin torque by means of their sliding motion within the DW. The DW line motion mobility at the precessional linear flow regime is not affected by substructure density, but at the steady linear flow regime it is dependent on the substructure density in a certain relationship according to which the mobility will be decreased by increasing the substructure density to efficiently eliminate the spin torque. At the depinning regime, since the additional pinning force exists due to the slide motion of substructures within the DW line, the depinning field will be increased almost linearly versus the substructure density, and our simulated results indicate that both pinning forces are in the same order. At the creep regime for $H \ll H_{c}$, the value of $U_{c 1} / k T$ will be reduced by increasing substructure density due to the thermal enhancement, while for $H \leq H_{c}$, the fitted exponent values of $\alpha$ are all around 1.25 which is in good agreement with that based on washboard potential model, and the ratios $U_{c 2} / U_{c 1}$ are also consistent with our previous work, indicating close disorder lengths in the two creep sub-regimes. 
Besides of the driving magnetic field, the spin-polarized current [150] as well the strain field [157] or spin wave [158] can also drive magnetic DWs, and then this work for dynamical properties of DW with substructures can be extended to those above different kinds of driving forces. The magnetoelastic effect is ignored in this work, and it may be large for other ultrathin magnetic film like cobalt ferrite [159]. One can expect that the magnetoelastic energy at substructures will be very high due to the fast varying magnetizations, which can drastically affect the statics and dynamics of DW, for example, deroughen the DW and increase the depinning field. It has reported that the strain line defect in thin magnetic film can deroughen DW and affect its creep behavior [136]. Furthermore, substructure as an additional freedom degree can also existed in other type of elastic string such as ferroelectric DW, and thus this work can also shed a light on the challenging problem for other elastic strings with substructures. 


\subsection{Current Induced Bloch Line Motion and Ultrafast Core Reversal}

\subsubsection{Introduction}

Domain wall substructures, as an internal freedom degree, can largely affect magnetic domain wall properties including the mobility, elasticity and so on as discussed in Sec. 3.2. Those substructures usually have many different magnetization forms as well as the corresponding names such as Bloch line, Neel line, vortex, and cross-tie, depending on the specific ferromagnetic material and the thickness. [153] For simplicity, we use the term Bloch line to generally treat all those substructures. Bloch lines structures in films have been widely investigated and lots of experimental observations for various magnetic systems by various methods have been reported, especially for ferromagnetic films with in-plane uniaxial magnetocrystalline anisotropy, such as Co [160], Fe [161], Permalloy [153], YIG [162, 163] and MnAs [164-166]. Actually, two types of domain walls, Bloch type and Neel type, either symmetric or asymmetric, are usually formed in films with in-plane uniaxial anisotropy. As the film thickness decreases, a wall type transition from Bloch to Neel type will take place, so that the Bloch line structure will be also transformed accordingly. The critical transition thickness is dependent of the anisotropy parameter $Q=K_{u} / K_{d}$, where $K_{u}$ is the anisotropy constant and $K_{d}=1 / 2 \mu_{0} \mathrm{M}_{\mathrm{s}}^{2}$ is the demagnetization energy [153]. Below the critical thickness, symmetric Neel wall will be formed, while above the critical value, symmetric (for $Q>1$ ) or asymmetric (for $Q<1$ ) Bloch walls will be formed. For symmetric Bloch wall, the magnetizations within the domain wall point out of the surface, and the Bloch line structure in the bulk or in the surface doesn't show much difference, and the Bloch line structure has been observed in MnAs film with strong in-plane anisotropy [164-166]. For 
asymmetric Bloch wall, however, the magnetizations of the domain wall will prefer to lie within the surface by forming the so-called Neel caps which can form cap switches and make the Bloch line structures in the bulk and in the surface quite different [153], leading to relatively complex Bloch lines as reported for Co [160] and Fe [161]films.

The magnetization processes of Bloch lines driven by magnetic field have been well studied several decades ago, and some applications based on Bloch lines have also been proposed, for example, vertical Bloch line memory that is based on the presence or absence of Bloch lines in band or bubble domain walls was proposed in 1980s [167-169]. In recent years, flux-closure magnetic dots based on cap switches motion in the thick Fe film surface [170] and vortex-core memory cell based on field driven ultrafast vortex core reversal in Permalloy nano-disk [40, 42, 171] accompany with strong spin wave radiation which plays a very important role in spin wave logics $[172,173]$ are also reported. Although lots of studies and applications have been made for magnetic field driven Bloch lines, Bloch lines motion induced by spin-polarized current which can act as an alternate way to drive domain walls $[6,18,26,174]$ was rarely investigated. Unlike those magnetic vortices constrained in nano-disks or nano-dots, Bloch lines in films can move more freely within domain walls, and in particular, all the Bloch lines can move in the same direction under current, which is quite different from the case driven by field. Therefore, understanding the unique properties of current induced Bloch line motion is fundamentally important and also crucial to future Bloch-line-based devices. In this section, current induced Bloch line motion in ideal magnetic film with relatively strong in-plane uniaxial anisotropy $(Q=2)$ are studied by micromagnetic simulations and theoretical analysis. As mentioned above, two major Bloch line structures exist for films 
with strong in-plane anisotropy, depending on the film thickness. For thick film or bulk (which can be treated as the extremely thick film), Bloch walls are preferred and the Bloch line structures are shown in Fig. 3.13 (a) and (b); for very thin film, Neel walls are favorable and the Bloch line structures are shown in Fig. 3.13 (c) and (d). For magnetic films with weak anisotropy, the Bloch lines for thin film also have similar structures as described by Fig. 3.13 (c) and (d), while for thick film, Bloch line structures are rather complex due to the existence of Neel caps, and then the studies of current induced motion properties for Bloch lines in thick films will be our future work and will not be present in this section.

\subsubsection{Current Induced Bloch Line Motion in Bulk}

The magnetization evolutions are described by the modified Landau-LifshitzGilbert equation, Eq. (2.1.11), including the conventional spin transfer torques as discussed in Sec. 2.1. Since it can be assumed that current induced Bloch lines motion in film or bulk is a universal behavior which should obey the same rule, artificial large magnetocrystalline anisotropy constant $K_{u}=\mu_{0} M_{s}^{2}$ (i.e., $Q=2$ ) is used for the ferromagnetic system with the other magnetic parameters set as: $M_{s}=8 \times 10^{5} \mathrm{~A} / \mathrm{m}$ and $A=1.3 \times 10^{-11} \mathrm{~J} / \mathrm{m}$. In order to systematically investigate that effect, we use a series of $\beta$ values in the range $-0.02 \sim 0.04$ with fixed $\alpha=0.02$. 

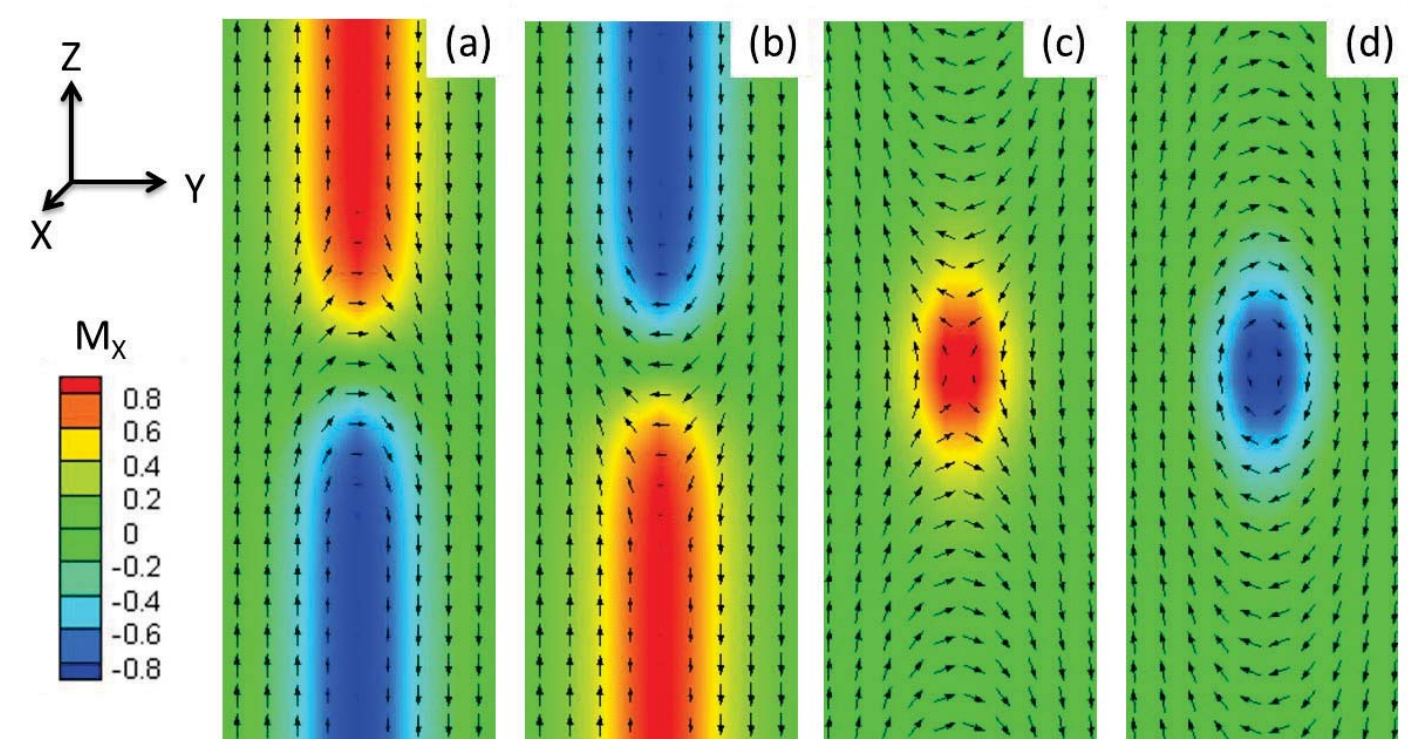

Figure 3.13 Magnetization structures of Bloch lines in (a)-(b) bulk and (c)-(d) thin film ferromagnetic systems. Small black arrows and color contours represent magnetization direction.

Unlike current induced domain wall motion in one-dimensional nanowires, twodimensional thin film or bulk allows Bloch lines to move in two dimensions and also allows spin-polarized current injected in two main directions, perpendicular to (in $\mathrm{Y}$ direction) and parallel with (in Z-direction) the domain wall. Therefore, Bloch lines velocities under various spin-polarized currents applied in the two main directions with different values of $\beta / \alpha$ that is assumed as a key factor to affect current induced Bloch lines motion behavior are computed from our micromagnetic simulations. Since Bloch line density in a domain wall is also an important factor to influence the Bloch lines' behavior, two different Bloch line densities, 2 Bloch lines per 400nm and 4 Bloch lines per 400nm, are also considered.

The Bloch lines structures in bulk are shown in Fig. 3.13 (a) and (b). Actually, Bloch line as a transition between two opposite orientated domain walls occupies two 
types of chiralities as well as two types of polarities. Fig. 3.13 (a) and (b) show a pair of Bloch lines with the same chirality but opposite polarities, so do Fig. 3.13 (c) and (d). For the study of Bloch lines motion in bulk, the current is applied in three modes, perpendicular mode (current in Y-direction, Fig. 3.14), parallel mode (current in Zdirection, Fig. 3.15) and mixed mode (current in YZ-direction, Fig. 3.16).

Fig. 3.14 shows the Bloch line motion properties under current applied perpendicular to the domain wall. It is noted that Bloch lines can move in two directions, along with and perpendicular to the domain wall. Our simulation results shown in Fig. 3.14 (b) and (c) indicate that these two components of Bloch line velocities, $v_{y}$ and $v_{z}$, are almost linearly related to both the value of $\beta / \alpha$ and the Bloch line density $N / 400 \mathrm{~nm}$, but with different extents: $v_{y}$, which also presents the domain wall velocity due to the interaction between Bloch lines and domain wall, is nearly equal to the current density value $u$, almost independent of the above two factors; $v_{z}$, however, shows a strong dependency on both factors, for instance, $\beta / \alpha=1$ makes $v_{z}=0$ while $\beta / \alpha>1$ (or $<1$ ) makes $v_{z}<0$ (or $>0$ ) with the sign determined by Bloch line chirality, and denser Bloch lines can reduce $v_{z}$. Actually, both the weak and strong dependency of $v_{y}$ and $v_{z}$ on the two factors arise from the same fact that the current induced spin transfer torques generated on the domain wall are evenly transferred to the Bloch lines, so that almost no torques are left on the domain wall, which makes the domain wall velocity or $v_{y}$ a constant value. Since the generated torques are related to $\beta / \alpha$ and will be evenly transferred to Bloch lines to drive them moving along the domain wall, the velocity component $v_{z}$ is dependent of $\beta / \alpha$ and $N$. 

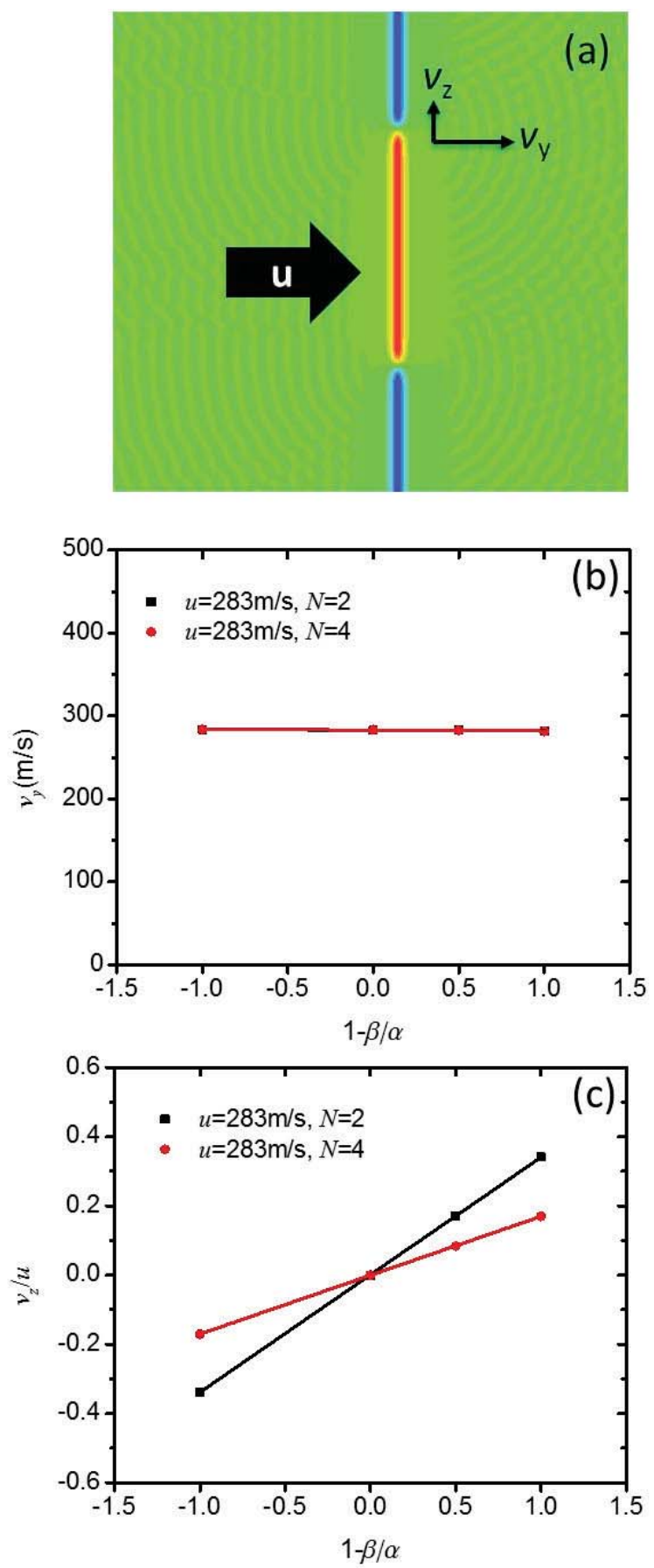

Figure 3.14 (a) Two Bloch lines motion induced by current $u=283 \mathrm{~m} / \mathrm{s}$ applied perpendicular to the domain wall. Black big arrow represents the current direction and black small arrows represent Bloch line motion direction. (b) Linear fitting of Bloch line velocity in Y-direction $v_{y}$ vs. $1-\beta / \alpha$ under Bloch line density $N=2$ and $4 / 400 \mathrm{~nm}$. (c) Linear fitting of Bloch line relative velocity in Z-direction $v_{z} / u$ vs. $1-\beta / \alpha$ under Bloch line density $N=2$ and $4 / 400 \mathrm{~nm}$. 
The existence of Bloch lines which can purge all the torques generated at the domain wall through slide motion within the wall makes the current induced domain wall motion quite different from that of domain wall without Bloch lines. First, the steady motion velocity for pure domain walls are $v=u \beta / \alpha$, while the velocity for domain wall with Bloch lines is always $v=u$, independent of $\beta / \alpha$ values. Second, inertia effect exists for pure domain wall, i.e., acceleration or deceleration takes place when injecting or removing current, while for domain wall with Bloch lines, no inertia effect exists and the velocity always keeps constant until current is removed and then the velocity will suddenly drop to zero. Third, the Walker threshold for domain wall with Bloch lines is much larger than that of pure domain wall, because of the purge or weakening of current induced spin transfer torques on domain wall segments between Bloch lines. Besides, the domain wall segments are often bent due to the unsynchronization of domain wall and Bloch lines motion, but such a bending doesn't take place for pure domain walls unless extrinsic pinning effect is included. In addition, spin waves can also be generated by the moving domain walls or Bloch lines, with the wavelength and frequency determined by the motion velocity. In spite of the small spin wave amplitude which is around $10^{-3} M_{s}$, the wave propagation forms are also different: only plane waves propagate for pure domain wall, while circular waves generated from Bloch lines also have a contribution. 

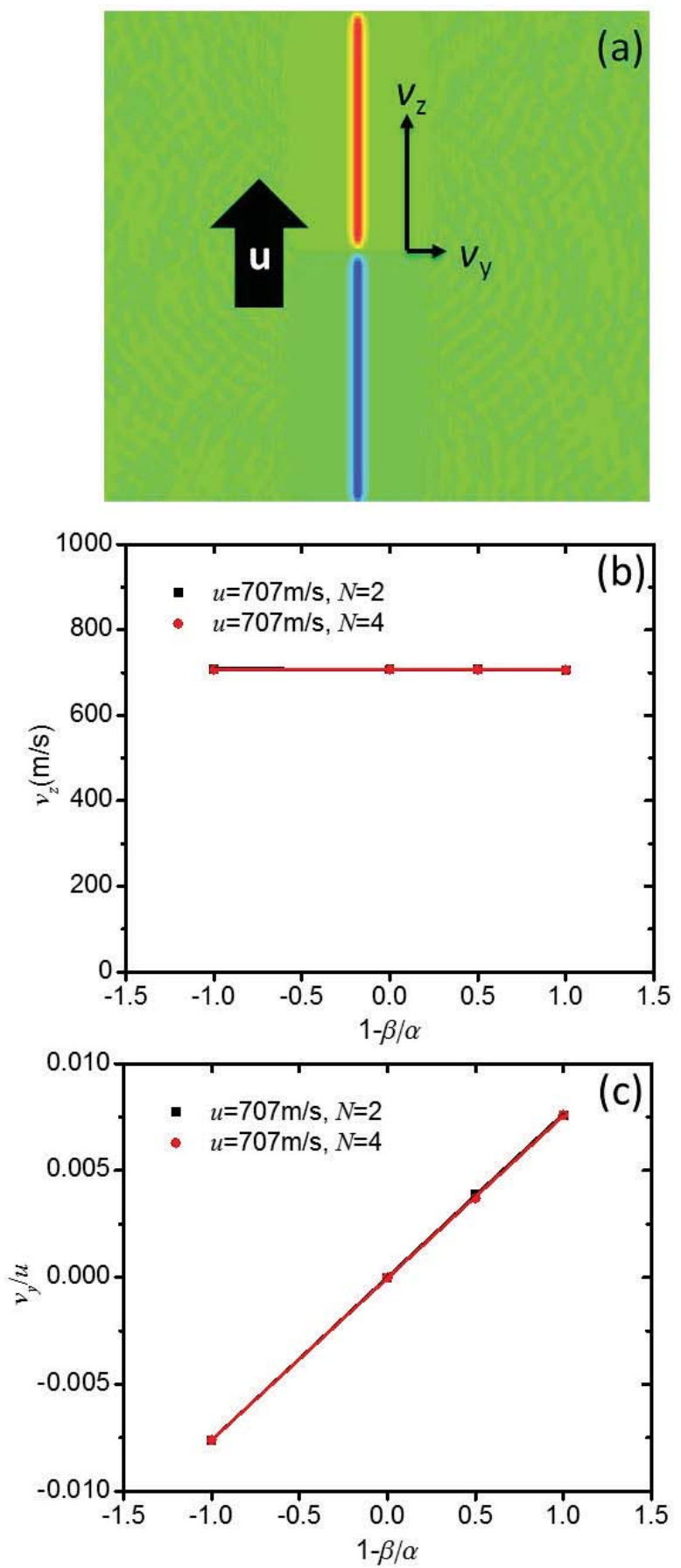

Figure 3.15 (a) Two Bloch lines motion induced by current $u=707 \mathrm{~m} / \mathrm{s}$ applied parallel with the domain wall. Black big arrow represents the current direction and black small arrows represent Bloch line motion direction. (b) Linear fitting of Bloch line velocity in Z-direction $v_{z}$ vs. 1- $\beta / \alpha$ under Bloch line density $N=2$ and $4 / 400 \mathrm{~nm}$. (c) Linear fitting of Bloch line relative velocity in Zdirection $v_{y} / u$ vs. $1-\beta / \alpha$ under Bloch line density $N=2$ and $4 / 400 \mathrm{~nm}$. 
When the current is applied parallel with the domain wall (in Z-direction), Bloch lines will move along the domain wall in the velocity $v_{z} \approx u$ with very small corrections dependent of $\beta / \alpha$, as shown in Fig. 3.15 (b). Unlike the domain wall motion in a nanowire with a steady velocity of $v=u \beta / \alpha$ accompany with an inertia effect, Bloch lines motion along a domain wall is almost independent of $\beta / \alpha$ and without an inertia effect through smearing its current-induced spin torques by exerting a drag force on the domain wall. Such a dragging force can drive the domain wall to move a slight distance in Y-direction, so that the Bloch lines accompany with the domain wall will have a very small velocity $v_{y}$, as shown in Fig. 3.15 (c). The value of $v_{y}$ is very small compared to $u$ and the ratio, $v_{y} / u$, is even less than $1 \%$. It is noted that such a small value of $v_{y} / u$ is almost independent of Bloch line density but depends on $\beta / \alpha$ very strongly. It should be also noted that for the parallel mode, the main motion velocity of Bloch lines, $v_{z}$, is independent of Bloch line chirality, but the moving direction perpendicular to the domain wall or the sign of the small motion velocity, $v_{y}$, is determined by the Bloch line chirality. When the Bloch line moves, circular spin waves are generated (see Fig. 3.15 (a)), but with an ignorable amplitude, $\sim 10^{-6} M_{s}$.

After obtaining the main behavior of current induced Bloch line motion with the two current injection modes discussed above, the mixed mode, i.e., current is applied in both $\mathrm{Y}$ and Z-direction simultaneously, is also computationally studied. Fig. 3.16 shows the Bloch line velocities for the mixed current mode, where $u_{z}$ is fixed to $141 \mathrm{~m} / \mathrm{s}$ and $u_{y}$ is varied in a certain range. For the velocity component in $\mathrm{Y}$-direction, $v_{y}$, its value is mainly determined by the current component $u_{y}$, as shown in Fig. 3.16 (b), and these values are almost independent of $\beta / \alpha$. For the velocity component in Z-direction, $v_{z}$, its 
value is contributed by both $u_{y}$ and $u_{z}$, as shown in Fig. 3.16 (c), and these values are explicitly dependent of $\beta / \alpha$. The dependency of $v_{z}$ on $u_{y}$, which can be represented by the slop $A$ shown in Fig. 3.16 (d), is the same with the case of perpendicular mode. All the above results demonstrate that for an arbitrary current (the mixed mode), the Bloch lines motion velocities are actually a linear superposition of the effect of $u_{y}$ (the perpendicular mode) and $u_{z}$ (the parallel mode).
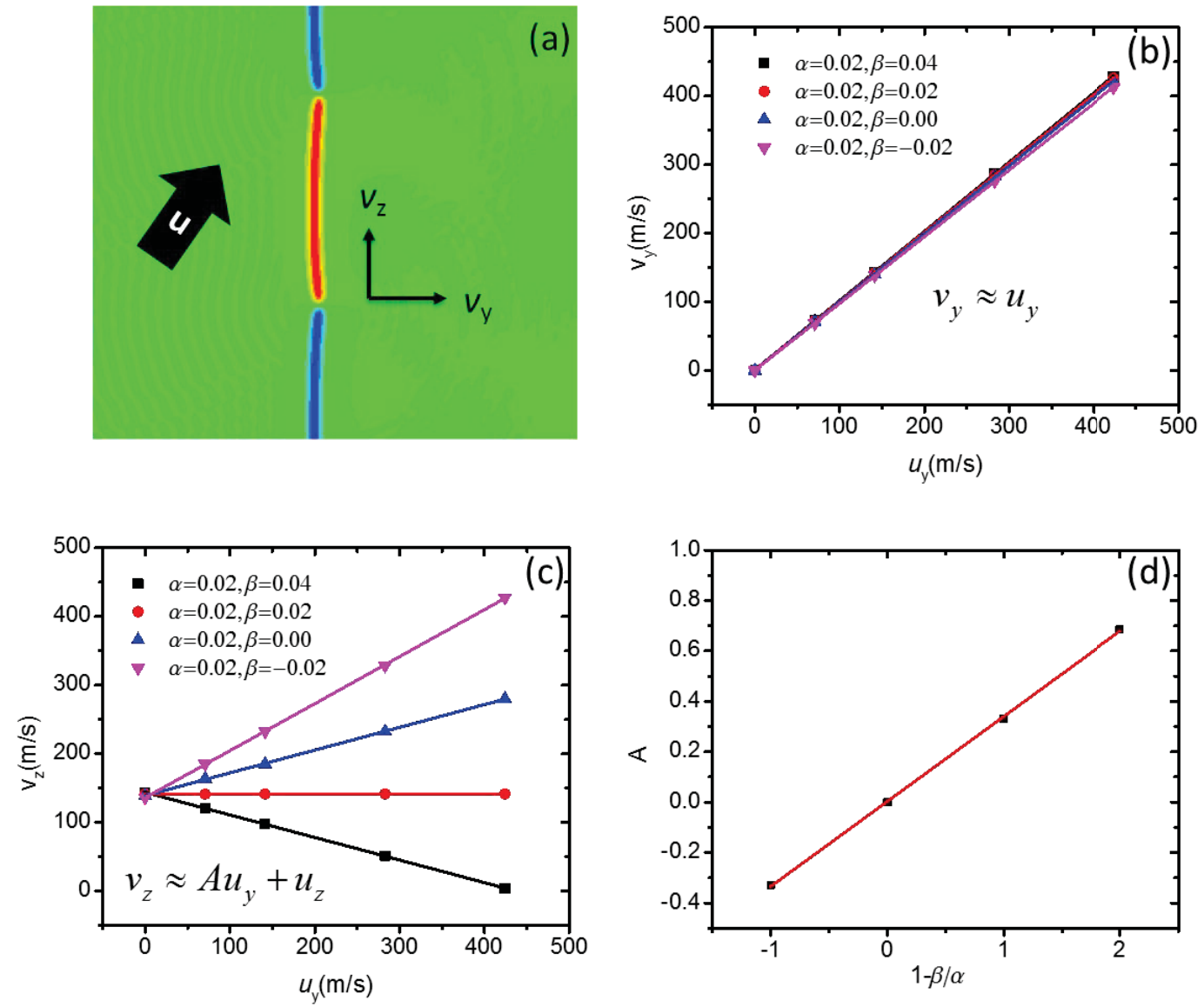

Figure 3.16 (a) Two Bloch lines motion induced by current applied in the mixed mode. Black big arrow represents the current direction and black small arrows represent Bloch line motion direction. (b) Linear fitting of Bloch line velocity in Y-direction $v_{y}$ vs. the current density in Ydirection $u_{y}$ with $\beta=0.04,0.02,0$ and -0.02 . (c) Linear fitting of Bloch line relative velocity in Zdirection $u_{z}$ with $\beta=0.04,0.02,0$ and -0.02 . (d) Linear fitting of the parameter $A$ vs. $1-\beta / \alpha$ according to the fitting results of (c). 


\subsubsection{Current Induced Bloch Line Motion in Thin Film}

Fig. 3.13 (c) and (d) show a pair of Bloch lines with the same chirality but opposite polarities in thin film, in analogy to the antivortex and vortex in nano-disk, respectively. For the computational study of current induced Bloch lines motion in thin film, similar simulation procedure as made for the above bulk cases is adopted, i.e., both the perpendicular and parallel current modes with the two key factors, $\beta / \alpha$ and Bloch line density, are taken into account. As expected, similar conclusions are obtained.

For the current applied perpendicular to the domain wall, as shown in Fig. 3.17, the perpendicular velocity of Bloch lines, $v_{y}$, which is also the domain wall velocity, is determined approximately by $v_{y} \approx u$, while the parallel velocity of Bloch lines, $v_{z}$, is strongly related to both $\beta / \alpha$ and Bloch line density denoted as $N / 400 \mathrm{~nm}$ as well as the Bloch line chirality. For the current applied parallel with the domain wall, as shown in Fig. 3.18, the parallel velocity of Bloch lines, $v_{z}$, is determined approximately by $v_{z} \approx u$, while the perpendicular velocity of Bloch lines, $v_{y}$, which is also the domain wall velocity, is very small and strongly related to $\beta / \alpha$ and Bloch line chirality but almost independent of Bloch line density. One can expect that for the Bloch lines motion under an arbitrary applied current (mixed mode), the velocity components can be obtained by dealing with the above two modes individually. 

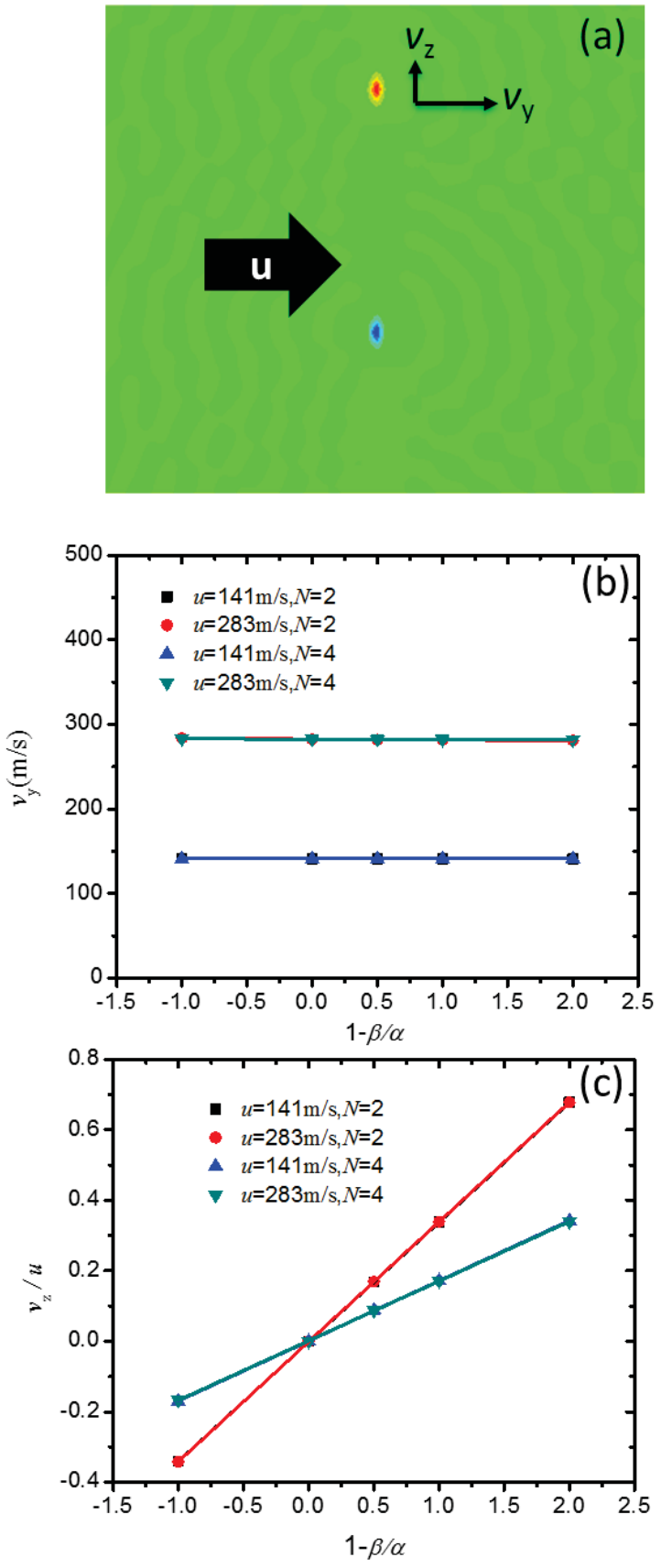

Figure 3.17 (a) Two Bloch lines motion induced by current applied perpendicular to the domain wall. Black big arrow represents the current direction and black small arrows represent Bloch line motion direction. (b) Linear fitting of Bloch line velocity in Y-direction $v_{y}$ vs. 1- $\beta / \alpha$ under Bloch line density $N=2$ or $4 / 400 \mathrm{~nm}$ and $u=141$ or $283 \mathrm{~m} / \mathrm{s}$. (c) Linear fitting of Bloch line relative velocity in Z-direction $v_{z} / u$ vs. $1-\beta / \alpha$ under Bloch line density $N=2$ or $4 / 400 \mathrm{~nm}$ and $u=141$ or $283 \mathrm{~m} / \mathrm{s}$. 

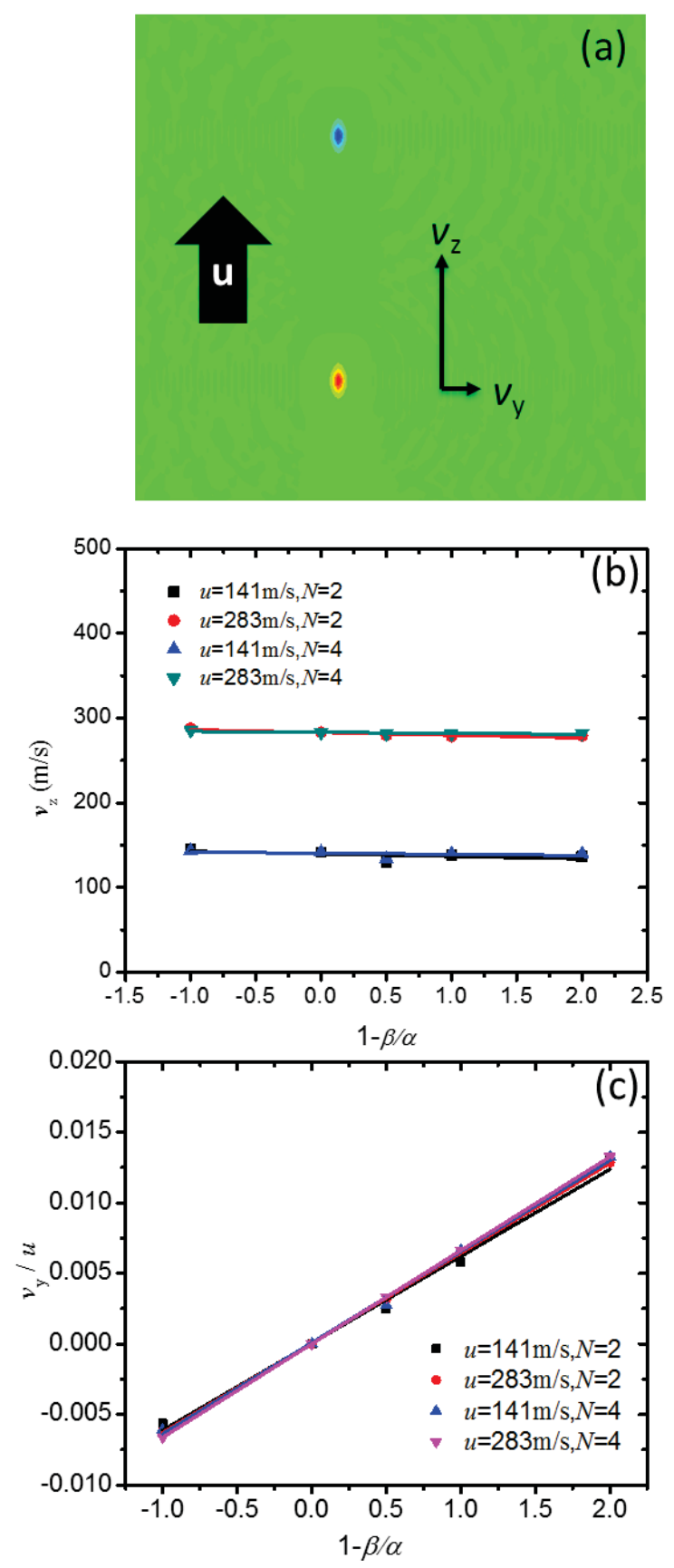

Figure 3.18 (a) Two Bloch lines motion induced by current applied parallel with the domain wall. Black big arrow represents the current direction and black small arrows represent Bloch line motion direction. (b) Linear fitting of Bloch line velocity in Z-direction $v_{z}$ vs. 1- $\beta / \alpha$ under Bloch line density $N=2$ or $4 / 400 \mathrm{~nm}$ and $u=141$ or $283 \mathrm{~m} / \mathrm{s}$. (c) Linear fitting of Bloch line relative velocity in Z-direction $v_{y} / u$ vs. $1-\beta / \alpha$ under Bloch line density $N=2$ or $4 / 400 \mathrm{~nm}$ and $u=141$ or $283 \mathrm{~m} / \mathrm{s}$. 


\subsubsection{Theoretical Analysis}

In order to better understand the current induced Bloch lines motion behaviors revealed by the above simulation results, theoretical analysis based on the modified Landau-Lifshitz-Gilbert equation is needed. As is indicated by the above results, the Bloch line behaviors in bulk and thin film are almost the same, and hence our theoretical analysis for understanding the universal properties focuses on the thin film system only.

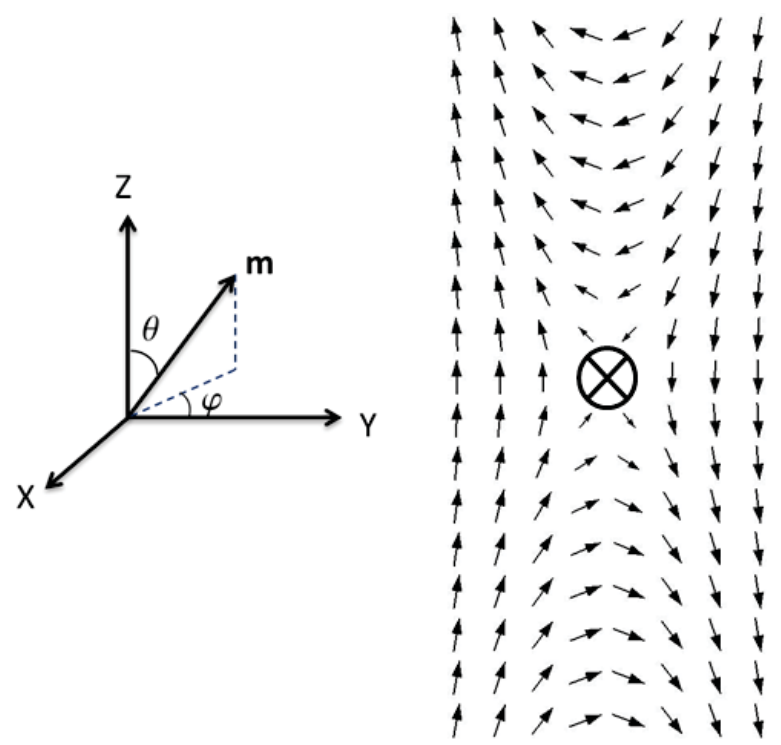

Figure 3.19 The cross type Bloch line structure and polar coordinate system with two main variables $\theta$ and $\varphi$.

Based on the polar coordinate where $\theta$ and $\varphi$ are two main variables as shown in Fig. 3.19, the total energy consisting of exchange energy, shape anisotropy energy and magnetocrystalline anisotropy energy can be given by

$$
E=\iint\left\{A\left[\left(\frac{\partial \theta}{\partial y}\right)^{2}+\sin ^{2} \theta\left(\frac{\partial \varphi}{\partial z}\right)^{2}\right]+\sin ^{2} \theta\left(K_{u}+K_{d} \sin ^{2} \varphi\right)\right\} d y d z,
$$


which is based on a certain approximation [153] stating that $\theta$ is $y$-dependent only and $\varphi$ is $z$-dependent only, with both of their values varying from $0^{\circ}$ to $180^{\circ}$. The variation of total energy density with respect to $\theta$ and $\varphi$ can be written as

$$
\left\{\begin{array}{l}
\frac{\delta e_{t o t}}{\delta \theta}=-2 A \frac{\partial^{2} \theta}{\partial y^{2}}+2 \sin \theta \cos \theta\left[K_{u}+K_{d} \sin ^{2} \varphi+A\left(\frac{\partial \varphi}{\partial z}\right)^{2}\right] \\
\frac{\delta e_{t o t}}{\delta \varphi}=2 \sin ^{2} \theta\left[K_{d} \sin \varphi \cos \varphi-A \frac{\partial^{2} \varphi}{\partial z^{2}}\right]
\end{array} .\right.
$$

The domain wall structure can be obtained by solving both $\delta e_{t o t} / \delta \theta=0$ and $\delta e_{t o t} / \delta \varphi=0$, and then we have

$$
\left\{\begin{array} { l } 
{ \operatorname { c o s } \theta = - \operatorname { t a n h } ( y / \lambda _ { Y } ) } \\
{ \operatorname { c o s } \varphi = - \operatorname { t a n h } ( z / \lambda _ { Z } ) }
\end{array} \text { with } \left\{\begin{array}{l}
\lambda_{Y}=\sqrt{A /\left(K_{u}+2 K_{d} \sin ^{2} \varphi\right)} \\
\lambda_{Z}=\sqrt{A / K_{d}}
\end{array}\right.\right.
$$

where $\lambda_{Y}$ and $\lambda_{Z}$ are Bloch line width parameters along $\mathrm{Y}$ and $\mathrm{Z}$ directions, respectively. When $\varphi=0^{\circ}$ or $180^{\circ}$, we can obtain the domain wall width parameter $\lambda_{D W}=\sqrt{A / K_{u}}$. The Bloch line energy is hence obtained by the integration in $\mathrm{Y}$ and Z-direction,

$$
\begin{aligned}
E & =\iint\left\{A\left[\left(\frac{\partial \theta}{\partial y}\right)^{2}+\sin ^{2} \theta\left(\frac{\partial \varphi}{\partial z}\right)^{2}\right]+\sin ^{2} \theta\left(K_{u}+K_{d} \sin ^{2} \varphi\right)\right\} d y d z \\
& =4 \sqrt{A K_{u}} \int \sqrt{1+\frac{2 K_{d}}{K_{u}} \operatorname{sech}^{2} \frac{z}{\lambda_{z}}} d z
\end{aligned}
$$

Since $Q=2$ for our thin film system, we have $\lambda_{Z}=\sqrt{2} \lambda_{D W}=2 \lambda_{Y}$, indicating that Bloch line is narrower than domain wall, which is consistent with our simulation results. The Bloch line energy is $E=8 \lambda_{z} \sqrt{A K_{u}} \sinh (1) \approx 9.4 \lambda_{z} \sqrt{A K_{u}}$ for $\lambda \in\left[-\lambda_{z},+\lambda_{z}\right]$. For current 
induced Bloch line motion, the two main current modes, perpendicular mode with $\mathbf{u}=[0$, $u, 0]$ and parallel mode with $\mathbf{u}=[0,0, u]$, are considered.

For the perpendicular mode with $\mathbf{u}=[0, u, 0]$, the modified Landau-LiffshitzGilbert equation in the polar coordinate can be written as

$$
\left\{\begin{array}{l}
\dot{\theta}=-\frac{\gamma}{M_{s} \sin \theta} \frac{\delta e_{t o t}}{\delta \varphi}-\alpha \sin \theta \dot{\varphi}-u \frac{\partial \theta}{\partial y}-\beta u \sin \theta \frac{\partial \varphi}{\partial y} \\
\sin \theta \dot{\varphi}=\frac{\gamma}{M_{s}} \frac{\delta e_{t o t}}{\delta \theta}+\alpha \dot{\theta}-u \sin \theta \frac{\partial \varphi}{\partial y}+\beta u \frac{\partial \theta}{\partial y}
\end{array} .\right.
$$

Since Bloch lines can move in two dimensions unlike domain walls, it can be assumed that the structures of Bloch lines will not be change during the motion process, i.e., the conditions of $\delta e_{t o t} / \delta \theta=0$ and $\delta e_{t o t} / \delta \varphi=0$ are always kept. Under that assumption, both Bloch line and domain wall will have the same velocity, and we have

$$
\left\{\begin{array}{l}
\left(1+\alpha^{2}\right) \dot{\theta}=\left(1+\alpha^{2}\right)\left(-\frac{\partial \theta}{\partial y} v_{y}\right)=-(1+\alpha \beta) u \frac{\partial \theta}{\partial y} \\
\left(1+\alpha^{2}\right) \sin \theta \dot{\varphi}=\left(1+\alpha^{2}\right) \sin \theta\left(-\frac{\partial \varphi}{\partial z} v_{z}\right)=(\beta-\alpha) u \frac{\partial \theta}{\partial y}
\end{array} .\right.
$$

Therefore, the velocity of Bloch line or domain wall in Y-direction is $v_{y}=\frac{1+\alpha \beta}{1+\alpha^{2}} u \approx u$, which is consistent with our simulation results shown by Fig. 3.14 (b), Fig. 3.16 (b), and Fig. 3.17 (b) for both the bulk and thin film system. Obviously, the velocity is also independent of Bloch line types and polarities. For the Bloch line velocity in Z-direction, since we have $\frac{\partial \theta}{\partial y}=\frac{\sin \theta}{\lambda_{Y}}$ and $\frac{\partial \varphi}{\partial z}=\frac{\sin \varphi}{\lambda_{Z}}$, the velocity is given by $v_{z}=\frac{\alpha-\beta}{1+\alpha^{2}} u \frac{\lambda_{Z}}{\lambda_{Y} \sin \varphi}$ which is $\varphi$-dependent. However, such a velocity is not correct for the ignorance of spin 
torques purge effect, i.e., the current induced spin torques $\dot{\varphi}=\frac{\beta-\alpha}{1+\alpha^{2}} \frac{u}{\lambda_{Y}}$ generated at domain wall segments between Bloch lines will be almost absolutely transferred to Bloch lines in order to keep the structure unchanged and then the velocity of Bloch lines in Zdirection will be higher due to the additional torques. Therefore, the torques along the domain wall will be redistributed by introducing a distribution function $P(\varphi)$, i.e., $\dot{\varphi}=P(\varphi) \frac{\beta-\alpha}{1+\alpha^{2}} \frac{u}{\lambda_{Y}}$, and then the velocity will be $v_{z}=P(\varphi) \frac{\alpha-\beta}{1+\alpha^{2}} u \frac{\lambda_{Z}}{\lambda_{Y} \sin \varphi}$. Since $v_{z}$ must be constant and independent of $\varphi$, we have $P(\varphi) \propto \frac{\lambda_{Y} \sin \varphi}{\lambda_{Z}}$. By introducing a dimensionless coefficient $\chi$, we have $P(\varphi)=\chi \frac{\lambda_{Y} \sin \varphi}{\lambda_{Z}}$. According to the conservation of spin transfer torques, $P(\varphi)$ must satisfy

$$
\int_{-\frac{L}{2 N}}^{\frac{L}{2 N}} P(\varphi) \frac{\beta-\alpha}{1+\alpha^{2}} \frac{u}{\lambda_{Y}} d z=\int_{-\frac{L}{2 N}}^{\frac{L}{2 N}} \frac{\beta-\alpha}{1+\alpha^{2}} \frac{u}{\lambda_{Y}} d z,
$$

where $L$ is the total domain wall length, $N$ the total Bloch lines number and then $L / N$ the average domain wall length occupied by one Bloch line. Therefore, we have

$$
\chi=\frac{\int_{-\frac{L}{2 N}}^{\frac{L}{2 N}} \frac{1}{\lambda_{Y}} d z}{\int_{-\frac{L}{2 N}}^{\frac{L}{2 N}} \frac{\sin \varphi}{\lambda_{Z}} d z}=\frac{\int_{-\tilde{L}}^{\tilde{L}} \sqrt{Q+2 \operatorname{sech}^{2} Z} d Z}{\int_{-\tilde{L}}^{\tilde{L}} \operatorname{sech} Z d Z}
$$

where $Q \equiv \frac{K_{u}}{K_{d}}=2, Z \equiv \frac{z}{\lambda_{z}}$ and $\tilde{L} \equiv \frac{L}{2 N \lambda_{z}}$. For our thin film system, $\tilde{L}=18$ and $\chi=16.6$ for $N=2$, while $L=9$ and $\chi=8.5$ for $N=4$. Therefore, the corrected velocity is 
given by $v_{z}=\frac{\alpha-\beta}{1+\alpha^{2}} \approx \chi \alpha\left(1-\frac{\beta}{\alpha}\right) u$, which is consistent with our simulation results shown by Fig. 3.14 (c), Fig. 3.16 (c)-(d), and Fig. 3.17 (c) for both the bulk and thin film system. In fact, for large $Q$ or $\tilde{L}, \chi \approx 2 \tilde{L} \sqrt{Q} / \pi \propto 1 / N$, and then $v_{z} \propto 1 / N$. It is noted that the velocity $v_{z}$ depends on the value of $\beta / \alpha$, and its sign depends on Bloch line chirality.

All the above analysis are based on the ideal assumption that all the spin transfer torques generated at domain walls are transferred to Bloch lines so that Bloch lines are shifted along domain walls with higher velocity while the domain wall velocity will be kept at a constant. In another word, the part of torques which should be used to accelerate or decelerate the domain wall velocity from $u$ to $u \beta / \alpha$ are now used to shift Bloch lines along the domain wall. Actually, the torques are partially transferred to Bloch lines rather than fully transferred, which causes the domain wall bended a little bit. By considering the small correction which represents the fraction of torques not fully transferred, denoted as $o_{1}$, we have $\dot{\varphi}=\frac{\beta-\alpha}{1+\alpha^{2}} \frac{u}{\lambda_{Y}}\left[1-o_{1}(N, u)\right]$, where $o_{1}(N, u)$ may depend on various factors like Bloch line density represented by $N$, or current density $u$, and such a small correction is introduced to deal with the velocity deviation from the ideal case. Under the above assumption, the velocity in Y-direction will be $v_{y} \approx u-o_{1}\left(1-\frac{\beta}{\alpha}\right) u$, which is linearly related with $1-\beta / \alpha$, consistent with the simulation results shown by Fig. 3.14 (b) and Fig. 3.17 (b). The small fractions $o_{1}$ obtained by the linear fitting are so small (less than $1 \%$ ) that they can be ignored. Since uncompleted torques transfer can cause a 
domain wall bending which may change the Bloch line structure and its width parameter $\lambda_{z}$, the values of $\chi$ from the simulation results in Tab. 3.3 will be deviated from our calculated values using Eq. (3.3.8) in a certain manner.

For the parallel mode with $\mathbf{u}=[0,0, u]$, similar analysis process with the perpendicular mode can be made. The modified Landau-Lifshitz-Gilbert equation in the polar coordinate can be written as

$$
\left\{\begin{array}{l}
\dot{\theta}=-\frac{\gamma}{M_{s} \sin \theta} \frac{\delta e_{t o t}}{\delta \varphi}-\alpha \sin \theta \dot{\varphi}-u \frac{\partial \theta}{\partial z}-\beta u \sin \theta \frac{\partial \varphi}{\partial z} \\
\sin \theta \dot{\varphi}=\frac{\gamma}{M_{s}} \frac{\delta e_{t o t}}{\delta \theta}+\alpha \dot{\theta}-u \sin \theta \frac{\partial \varphi}{\partial z}+\beta u \frac{\partial \theta}{\partial z}
\end{array} .\right.
$$

Under the same assumption that the Bloch lines structures are unchanged during the motion process, we have

$$
\left\{\begin{array}{l}
v_{y}=u \frac{\beta-\alpha}{1+\alpha^{2}} \frac{\lambda_{Y}}{\lambda_{Z}} \sin \varphi \\
v_{z}=\frac{1+\alpha \beta}{1+\alpha^{2}} u \approx u
\end{array} .\right.
$$

Therefore, the Bloch line velocity in Z-direction described by $v_{z} \approx u$ is consistent with our simulation results shown by Fig. 3.15 (b) and Fig. 3.18 (b) for the bulk and thin film system, respectively. The velocity derivation from u can also be treated by introducing a small correction $o_{2}(N, u)$ as what we did above for the perpendicular mode, and then we have $v_{z} \approx u-o_{2}\left(1-\frac{\beta}{\alpha}\right) u$, where $o_{2}$ is around $1 \%$ according to our simulation results. For the Bloch line velocity in Y-direction, if the torque redistribution effect is further considered as the perpendicular mode, i.e., the torques generated at Bloch lines are 
completely transferred to domain walls, the velocity will be $v_{y} \approx \alpha \chi^{-1}\left(1-\frac{\beta}{\alpha}\right) u$, where $\chi$ is described by Eq. (3.3.8). Since $\chi$ is a relatively large value, the velocity $v_{y}$ is therefore ignorable, which, however, is inconsistent with our simulation results. For that reason, we use a general form $v_{y} \approx \alpha \kappa\left(1-\frac{\beta}{\alpha}\right) u$. It is noted from Tab. I that the values of $\kappa$ is much larger than $\chi^{-1}$, indicating that the Bloch line motion in Y-direction or the Bloch line induced domain wall motion is not mainly due to the spin torques transfer effect, and the drag force exerted by Bloch lines on domain wall segments seems more important. Once the domain wall segment is bent by the drag effect, the intensive string vibration takes place on the domain wall segment which is thereafter dragged to the new position. Since there is an up limit for the Bloch line velocity in Y-direction according to Eq. (3.3.10), $\kappa$ will also have an up limit value, $\kappa_{\max }=0.5$.

Table 3.3 $\chi$ and $\kappa$ values under Bloch line density $N=2$ and $4(/ 400 \mathrm{~nm})$ for bulk and thin film systems obtained from the simulation results.

\begin{tabular}{cccc}
\hline & & $N=2$ & $N=4$ \\
\hline \multirow{2}{*}{ Bulk } & $\chi$ & 17.0 & 8.5 \\
& $\kappa$ & 0.38 & 0.38 \\
\hline \multirow{2}{*}{ Film } & $\chi$ & 17.0 & 8.5 \\
& $\boldsymbol{\kappa}$ & 0.31 & 0.32 \\
\hline
\end{tabular}

Finally, the main conclusion obtained from our theoretical analysis is summarized in the following. For the perpendicular mode with current applied in Y-direction, the current induced Bloch line velocity is given by 


$$
\left\{\begin{array}{l}
v_{y} \approx u \\
v_{z} \approx \alpha \chi\left(1-\frac{\beta}{\alpha}\right) u
\end{array}\right.
$$

where $\chi$ is given by Eq. (3.3.8) and strongly dependent of Bloch line density. For the perpendicular mode with current applied in Y-direction, the current induced Bloch line velocity is given by

$$
\left\{\begin{array}{l}
v_{y} \approx \alpha \kappa\left(1-\frac{\beta}{\alpha}\right) u \\
v_{z} \approx u
\end{array}\right.
$$

where $\kappa$ is less than 0.5 and weakly dependent of Bloch line density. All the calculated results are in good agreements with our simulation results.

\subsubsection{Ultrafast Reversal of Bloch lines in Thin Film}

Circular and cross type Bloch lines in thin film can also be treated as vortex or antivortex confined within the domain wall according to their structures shown by Fig. 3.13 (c) and (d). Similar structures usually promise similar properties. Especially for the ultrafast reversal of core polarity manifested by vortex/antivortex in soft magnetic nanodisk under field or current pulse $[40,42,171]$, the Bloch line polarity may also be reversed by applying current or field pulse. Compared with vortex/antivortex , Bloch lines seem to have more advantages, for example, unlike the vortex/antivortex in isolated nano-disk which can only be located in the center of the disk, Bloch lines which can be moved by current or field will have more freedom, and also the size of Bloch line is smaller due to the high in-plane uniaxial anisotropy. Therefore, the computational study of Bloch line reversal behavior stimulated by field and current is meaningful. In the 
following, the Bloch line reversal under magnetic field pulse in both perpendicular and parallel modes as well as under current pulse in perpendicular mode is presented based on our micromagnetic simulations.

Once the large magnetic field is applied perpendicular to the domain wall, a new pair of Bloch lines with opposite polarity will be created shortly near the original Bloch line. Fig. 3.20 shows the cross type Bloch line reversal process under a large magnetic field pulse. It is noted that a new pair of Bloch lines, a circular type and a cross type Bloch line, both with positive polarity, are created beside the original cross type Bloch line with negative polarity in 120ps, as shown in Fig. 3.20 (b). After that, the original cross type Bloch line is annihilated by the adjacent circular type new Bloch line, accompany with strong spin waves generated by the annihilation process, as shown in Fig. 3.20 (c). In the end, only the new cross type Bloch line with positive polarity is left, which is shown by Fig. 3.20 (d). Thus, only the polarity of the Bloch line is reversed under the field pulse perpendicular to the domain wall, which is quite similar with the reversal process for vortex/antivortex in nano-disk.

For the large magnetic field applied parallel with the domain wall, the reversal process seems different. Fig. 3.21 (b) shows that such a large field is able to flip the magnetization at the domain wall, and then a different type Bloch line with the same polarity can be created beside of the original Bloch line. Fig. 3.21 (c) shows a new circular type Bloch line with positive polarity is created at the adjacent of the original cross type Bloch line with positive polarity, and then the two Bloch lines will annihilate each other to finally preserve the circular Bloch line with negative polarity at the other side of the original one, as shown by Fig. 3.21 (d). Thus, both the Bloch type and its 
polarity will be reversed by the field applied parallel with the domain wall, which is quite different from the reversal process for the perpendicular case and the vortex/antivortex case. It is worth noted that the Bloch line chirality is not change during such reversal process.

In addition to the magnetic field, the spin-polarized current can also reverse Bloch lines. Fig. 3.22 shows the reversal process of a cross type Bloch line with positive polarity under the current pulse applied perpendicular to the wall. It can be noted that such a reversal mechanism is similar with the parallel field case described in Fig. 3.21, which makes much sense because the magnetization of domain wall will be flipped in both cases. Thus, both the Bloch type and its polarity will be reversed by the current pulse applied perpendicular with the domain wall, different from the field induced ultrafast reversal of vortex/antivortex in nano-disk.
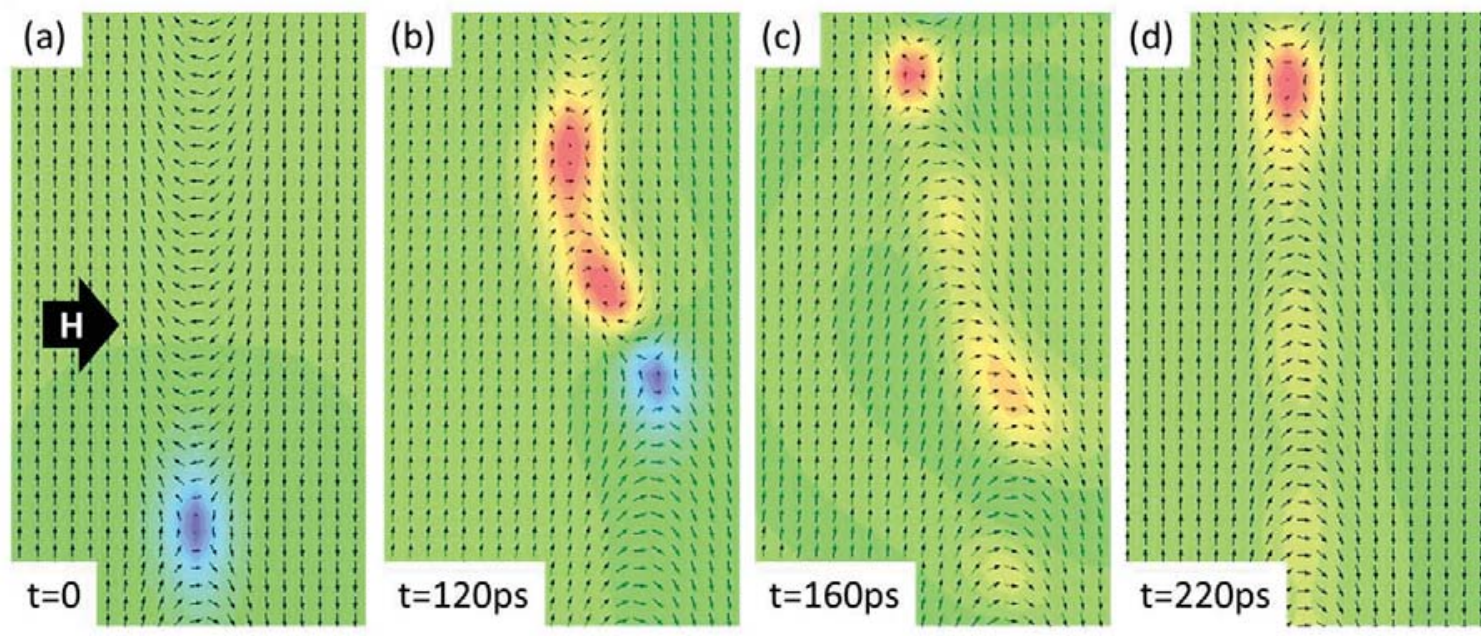

Figure 3.20 Magnetization structure for the ultrafast reversal process of the cross type Bloch line with negative polarity in (a) $t=0$, (b) $t=120 p s$, (c) $t=160 p s$ and (d) $t=220 p s$ under the magnetic field pulse with amplitude 2000Oe and duration 160ps applied perpendicular to the domain wall. Small black arrows and color contours represent magnetization direction. 

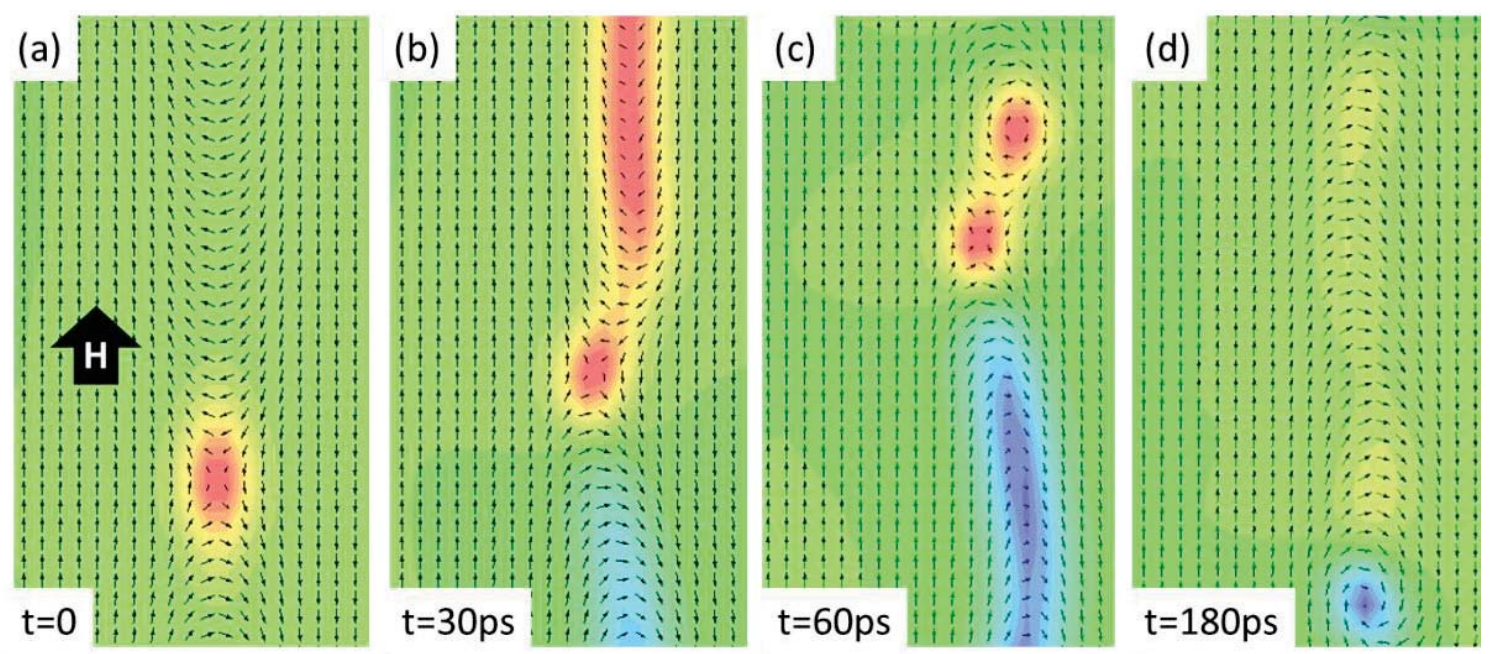

Figure 3.21 Magnetization structure for the ultrafast reversal process of the cross type Bloch line with positive polarity in (a) $t=0$, (b) $t=30 p s$, (c) $t=60 p s$ and (d) $t=180 p s$ under the magnetic field pulse with amplitude 2000Oe and duration 80ps applied parallel with the domain wall. Small black arrows and color contours represent magnetization direction.
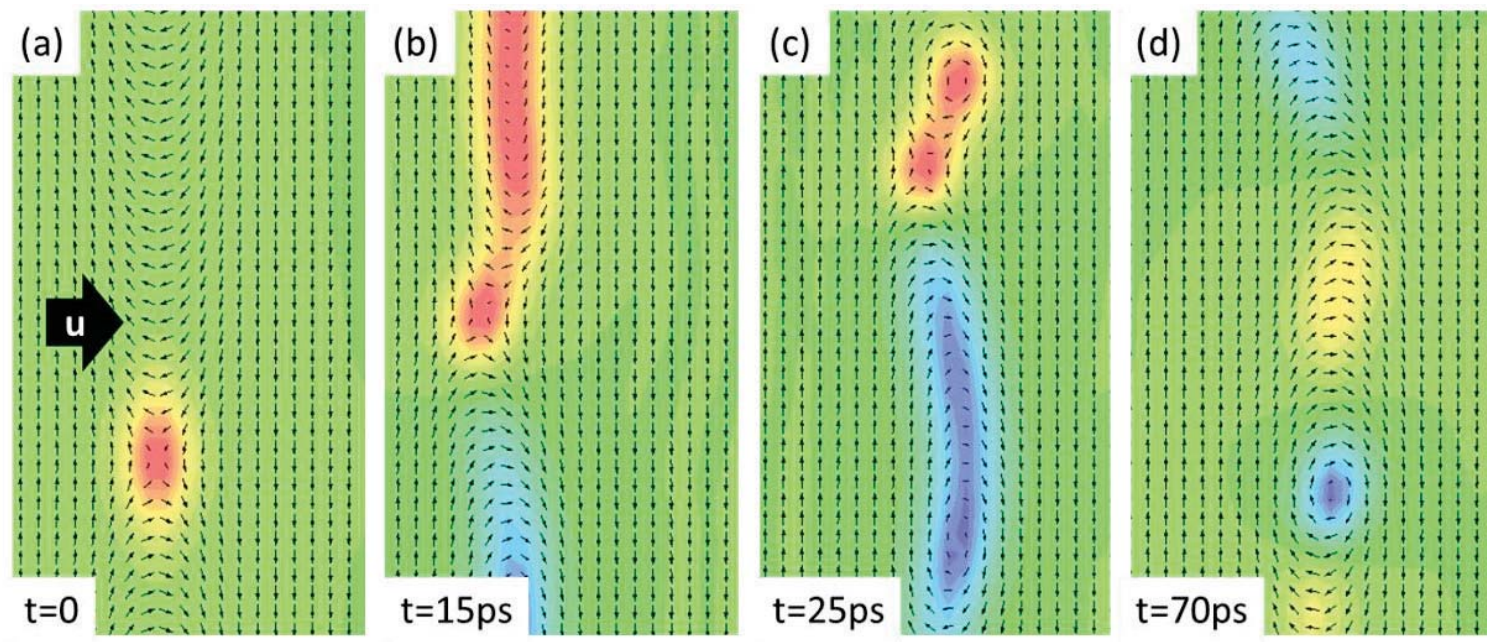

Figure 3.22 Magnetization structure for the ultrafast reversal process of the cross type Bloch line with positive polarity in (a) $t=0$, (b) $t=15 p s$, (c) $t=25 p s$ and (d) $t=70 p s$ under the spin-polarized current pulse with amplitude $\mathrm{u}=2000 \mathrm{~m} / \mathrm{s}$ and duration $60 \mathrm{ps}$ applied perpendicular to the domain wall. Small black arrows and color contours represent magnetization direction. 
For the current pulse applied parallel with domain wall, our simulation results show that the Bloch lines are difficult to be reversed, although the reversal mechanism is similar with the perpendicular field case shown in Fig. 3.20. Thus, in analogy to the ultrafast reversal of vortex/antivortex in nano-disk, Bloch lines in thin film can also be ultrafast reversed by magnetic field and spin-polarized current, but according to two different mechanisms.

\subsubsection{Conclusion}

In conclusion, current induced Bloch line motion in bulk and thin film with high in-plane uniaxial anisotropy has been investigated by both theoretical calculations and micromagnetic simulations in this section. Two main current modes are considered, one is for current applied perpendicular to the domain wall with $\mathbf{u}=[0, u, 0]$, the other is for current applied parallel with the domain wall $\mathbf{u}=[0,0, u]$. Both the bulk and thin film systems exhibit almost the same properties of current induced Bloch line motion. The Bloch line velocity parallel with current $\mathbf{u}$ for both modes is $v_{\|} \approx u$, which is independent of Bloch line types and densities, while the Bloch line velocity perpendicular with $\mathbf{u}$ is $v_{\perp} \approx \chi(\alpha-\beta) u$ for the perpendicular current mode and $v_{\perp} \approx \kappa(\alpha-\beta) u$ for the parallel current mode, where $\chi$ is almost inversely proportional to Bloch line density and $\kappa$ is weakly dependent of Bloch line density. Both the theoretical calculations and micromagnetic simulations agree with each other very well. Besides, ultrafast reversal of Bloch line in thin film is also computationally investigated. Our simulation results show that, in analogy to the ultrafast reversal of vortex/antivortex in nano-disk, Bloch lines in 
thin film can also be ultrafast reversed by magnetic field and spin-polarized current, but with two different mechanisms. Our study on current induced Bloch lines motion as well as the ultrafast Bloch line reversal can shed a light on future Bloch-line-based devices in spintronics. 


\subsection{Future Research}

1. Like magnetic field, spin-polarized current can also act as a driving force to make a one-dimensional domain wall line creep, depin and flow in thin film. Especially for the current induced domain wall creep near the depinning force, the creep exponent $\alpha$ is still not determined. Since the determination of $\alpha$ depends on the exact value of the depinning force which must be determined from the depinning regime, the current induced depinning behavior should be investigated first. Therefore, we will employ micromagnetic modeling to computationally study the current induced domain wall line behaviors in creep and depinning regime and then determine both the depinning exponent $\beta$ and creep exponent $\alpha$ which we suppose are the same with the magnetic field case. Experimental studies for those topics are still lacking, since the joule effect caused by the current have a great influence on the system temperature.

2. For domain wall line with substructures, the roughness, creep, depinning and flow behaviors under spin-polarized current are rarely studied either experimentally or computationally. We will use micromagnetic simulations to study those topics just like what we did in Sec. 3.2 for the magnetic field case.

3. Since domain all substructures usually exhibit strong elastic energy due to the magnetostriction effect, which results in a strong coupling between substructures and pinning sites and then makes the domain wall line more roughened, we will use micromagnetic simulations to study the roughening effect caused by elastically coupled substructures. 
4. Current induced Bloch line and cap switches motion for film with weak in-plane anisotropy will be also computationally studied. 


\section{Chapter 4. Magnetic Domain Walls in Nanowires ${ }^{\S}$}

\subsection{Nanometer Magnetic Transverse Domain Wall Logic}

\subsubsection{Introduction}

Magnetic field or spin-polarized current induced magnetic domain wall (DW) motion and DW transformation in planar magnetic wires plays a crucial role in the development of magnetic memory and logic elements. [19, 21, 34] Two types of DWs exist in planar soft magnetic wires, vortex DWs and transverse DWs, both of which can be treated as information carriers due to their modulatable chiralities or polarities. For vortex DWs, it has been reported that the vortex core polarity can be reversed by spinpolarized current [20] and the vortex DWs can also be transformed into transverse DWs $[13,175]$. Usually, vortex DWs as well as the relevant transformations are existed in relatively wide wires, and in very narrow wires, the transverse DWs are stable. For transverse DWs, two kinds of chiralities exist and it has been observed that the chirality reversal can be induced by a proper stimuli, such as external magnetic field [16], spinpolarized current [36] and current induced Oersted field [176]. It has also been reported that the spin-orbit torque effect or Rashba effect, besides of the spin transfer torque effect, can also lead to a transverse DW flipping. [29] The controllable chirality combined with the small size of transverse DWs in very narrow wires not only provide potential applications to achieve high density information storage, but also reveal the possibility to develop nanometer magnetic DW logic based on transverse DWs.

\footnotetext{
$\$$ The material contained in Section 4.2 of this chapter was previously partially published in Journal of Applied Physics, 2012. 112(8): p. 083903 by Geng, Liwei D. and Jin, Yongmei M.. The material contained in Section 4.3 of this chapter was previously published in Journal of Magnetism and Magnetic Materials, 2015. 393(0): p. 121-126 by Geng, Liwei D. and Jin, Yongmei M.. See Appendix C and $\mathrm{D}$ for documentations of permission to republish this material.
} 
Submicrometer magnetic logic circuits including NOT and AND gates based on DW propagation around a planar wire loop under rotating magnetic field taken also as the clock signal have been reported. [32, 34] However, it seems that such submicrometer logic elements can't be directly miniaturized and applied to nanometer wires or nanowires in which only transverse DWs exist. Rather than annihilating each other, winding transverse DWs prefer to pile up in a nanowire, according to our results in Sec. 4.2. Since all the transverse DWs generated by the NOT gate [34] with an enlarged central stub are winding DWs, they will be piled up together when encountering in nanowires, unlike the relatively wide wires in which DWs annihilation always happens. As is expected, those transverse DWs will be pinned and piled up at the junctions of AND or Cross-over circuit and will even accumulate further under the rotating field to eventually invalidate the functions of such logic circuits. Besides, the non-annihilation nature of winding transverse DWs also forbids the data clear-up process in the nanowire circuits. Take the NOT-based shift register [32] for example, our simulation results show that when two winding transverse DWs meet at the junction of a NOT gate in the nanowire, a $360^{\circ} \mathrm{DW}$ forms in the data clear-up process, as shown in Fig. 4.1.1 (a). Since unwinding transverse DWs will always annihilate each other in nanowires, all the above problems can be solved by introducing them. Thus, a novel data generator which generates unwinding transverse DWs is needed for miniaturized circuits.

Regardless of the above limitations in nanometer circuits, there are also other disadvantages for those submicrometer logic circuits [34]. For example, an inherent T/2 delay for DW propagation is associated with all NOT gates, resulting in a nonsynchronized signal when integrated with other circuits like AND or OR, which makes 
the external rotating field unable to be taken as a clock signal. [177] Besides, the information bit definitions for ' 0 ' and ' 1 ' are different for the logic gates and data input generator [34], and such a dual definition makes the integration of circuits difficult. Actually, both of the above bit definitions are based on domain magnetization rather than DW chirality. Since the chirality of a transverse DW can be modulated by some proper stimuli which does not require T/2 delay, a novel transverse DW-based logic at nanoscale may avoid such above disadvantages existed in submicrometer magnetic logic. This paper will employ micromagnetic simulations based on Landau-Lifshitz-Gilbert equations to propose novel nanometer logic elements based on transverse DWs by attempting avoiding the above disadvantages existed for submicrometer magnetic DW $\operatorname{logic}[175]$.

\subsubsection{Micromagnetic Modeling}

According to our micromagnetic model, magnetic domain structure in nanowires is described by magnetization field $\mathbf{M}(\mathbf{r})=M_{\mathrm{s}} \mathbf{m}(\mathbf{r})$, where $M_{\mathrm{s}}$ is saturation magnetization and $\mathbf{m}(\mathbf{r})$ is the magnetization directional unit vector field whose value is 0 outside nanowire circuits. The total system free energy is a functional of $\mathbf{m}(\mathbf{r})$ and is evaluated as a sum of magnetic anisotropy energy, exchange energy, magnetostatic energy, and external magnetic energy, [178] where the long-range magnetostatic energy is calculated in reciprocal space using the Fourier transform of the magnetization field: [178] 


$$
\begin{aligned}
E & =\int K\left\{1-[\mathbf{p}(\mathbf{r}) \cdot \mathbf{m}(\mathbf{r})]^{2}\right\} d^{3} r \\
& +\int A[\operatorname{grad} \mathbf{m}(\mathbf{r})]^{2} d^{3} r+\frac{1}{2} M_{s}^{2} f \frac{d^{3} k}{(2 \pi)^{3}} 4 \pi \frac{|\mathbf{k} \cdot \mathbf{m}(\mathbf{k})|^{2}}{k^{2}} \\
& -M_{s}^{2} \int \mathbf{h}_{\text {ext }} \cdot \mathbf{m}(\mathbf{r}) d^{3} r
\end{aligned}
$$

where $K$ is the magnetocrystalline anisotropy constant, $\mathbf{p}(\mathbf{r})$ the magnetic easy direction, $A$ the exchange stiffness constant, $\mu_{0}$ the vacuum permeability, $\mathbf{h}_{e x t}$ the external magnetic field, $f$ is evaluated as a principal value excluding the point $\mathbf{k}=\mathbf{0}$, $\mathbf{M}(\mathbf{k})=\int \mathbf{M}(\mathbf{r}) e^{-i \mathbf{k} \cdot \mathbf{r}} d^{3} r$, and $\mathbf{n}=\mathbf{k} / k$ is a unit directional vector in reciprocal space. The evolution of magnetic domain structure is driven by the thermodynamic driving force $\mathbf{H}_{\text {eff }}=-\delta E / \delta \mathbf{M}$, the variational derivative of the free energy in Eq. (4.1.1) with respect to the magnetization field variable, and is described by the Landau-Lifshitz-Gilbert equation, [178]

$$
\frac{\partial \mathbf{m}(\mathbf{r}, t)}{\partial t}=\gamma \mathbf{H}_{\text {eff }} \times \mathbf{m}-\alpha \mathbf{m} \times \frac{\partial \mathbf{m}(\mathbf{r}, t)}{\partial t}
$$

where $\gamma$ is the gyromagnetic ratio, and $\alpha$ is the damping parameter.

Equation (4.1.2) is numerically solved for all the given nanowire geometries in this paper. Nanowire thickness is $4 \mathrm{~nm}$. The width of nanowire loop used to transport DWs is $80 \mathrm{~nm}$. The sizes and structures for the transverse DW-based logic elements are shown in Tab. I. The material parameters of Permalloy $\mathrm{Ni}_{80} \mathrm{Fe}_{20}$ are used in the simulations: $M_{\mathrm{s}}=860 \mathrm{kA} / \mathrm{m}$, magnetocrystalline anisotropy $K=0$, and exchange constant $A=1.3 \times 10^{-11} \mathrm{~J} / \mathrm{m}$. [179] An elliptical counterclockwise rotating magnetic field $\mathbf{H} / M_{s}=\left[h_{x} \cos \left(2 \pi t^{*}\right), h_{y} \sin (2 \pi t *)\right]$ is applied to the circuits. 


\subsubsection{Results and Discussion}

\subsubsection{Data Generator}

Magnetic DWs, either transverse or vortex type, can be generated by rotating magnetic field acting on a large, magnetically soft region [34] or by current-pulsecarrying wire injected at the end of a magnetic wire $[19,180]$. Good data selectivity has been achieved by the former method. Reliable generating of winding transverse DWs from large hexagonal generator under rotating magnetic field cycles was demonstrated by our previous work [179]. As mentioned above, unwinding transverse DWs generation is required for nanometer logic circuits, or the DWs will be piled up at the element junction to affect corresponding functions. Fig. 4.1.1 (a) shows the formation of one $360^{\circ} \mathrm{DW}$ from two winding transverse $180^{\circ} \mathrm{DWs}$ at the junction of a NOT gate in the data clear-up process of the miniaturized shift register based on NOT gates [32], i.e., the unwanted transverse DWs pile-up makes the clear-up process failed. However, unwinding transverse DW pair will be annihilated instead of piled up, which can be shown by Fig. 4.1.1 (b). Thus, in order to avoid DWs pile-up, the unwinding DW generator is needed.

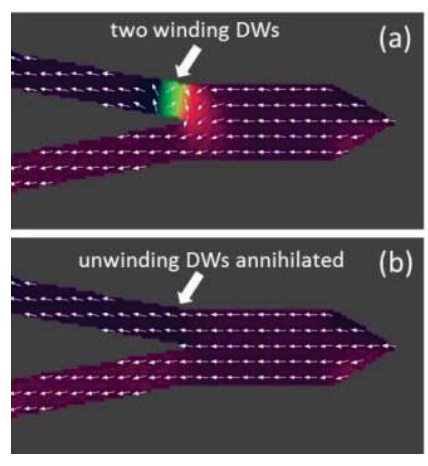

Figure 4.1.1 (a) Simulated domain structure of two winding transverse DWs piled up at the junction of one NOT gate by forming a $360^{\circ}$ DW. (b) Simulated domain structure of two unwinding transverse DWs annihilated at the junction due to the opposite chirality. Small white arrows and color contours within the nanowire represent magnetization direction. 
Since shape-isotropic wall generator always produce winding DWs due to the large soft region in which magnetizations prefer to follow external rotating magnetic field to output winding DWs, a shape-anisotropic wall generator may help produce unwinding transverse DWs. Fig. 4.1.2 shows a bar-shaped wall generator with magnetization within the bar always pointing 'down'. Unlike the shape-isotropic wall generator, the bar-shaped design actually restricts the magnetization responding under rotating field, i.e., the magnetization within the bar will never follow the rotating field unless very large field is applied. Fig. 4.1.2 (a)-(e) show the process of a pair of unwinding transverse DWs generated by a bar-shaped wall generator under rotating field with amplitude of $h_{x}=0.06$ and $h_{y}=0.03$ in one cycle. In the first half cycle (Fig. 4.1.2 (a)-(b)), the 'down' magnetization within the bar doesn't follow the rotating driving field, which forces the first transverse DW to point 'down' at the junction B between the bar and nanowire conduit, generating a counterclockwise (ccw) head-to-head (hh) transverse DW, denoted as W1, pushed out from B by the field after the first half cycle, unlike the shape-isotropic one which will produce a clockwise $(\mathrm{cw})$ hh transverse DW after the first half cycle [179]. In the second half cycle (Fig. 4.1 .2 (c)-(e)), W1 pinned at corner $\mathrm{C}$ will be transported along the nanowire loop by passing through corner $\mathrm{D}$, and the second wall W2 which is cw tail-to-tail (tt) transverse DW is generated from B and eventually pinned at corner $\mathrm{C}$ after the second half cycle. Therefore, a pair of unwinding transverse DWs, W1 and W2, are generated in one rotating cycle from the bar-shaped wall generator. When the rotating field amplitude $h_{x}<0.048$, no any DWs will be generated. The modulation of presence and absence of DW pairs makes the bar-shaped wall generator a 
data generator which is in good agreement with the submicrometer data generator [34] while still valid in nanowires. Based on our simulation results, adjusting the width of the bar can modulate the critical rotating field to generate or not generate DWs, for example, DWs can be generated by lower field for wider bar. As is expected, if the magnetization within the bar points 'up', a cw-hh and a ccw-tt transverse DW will be generated in one cycle.
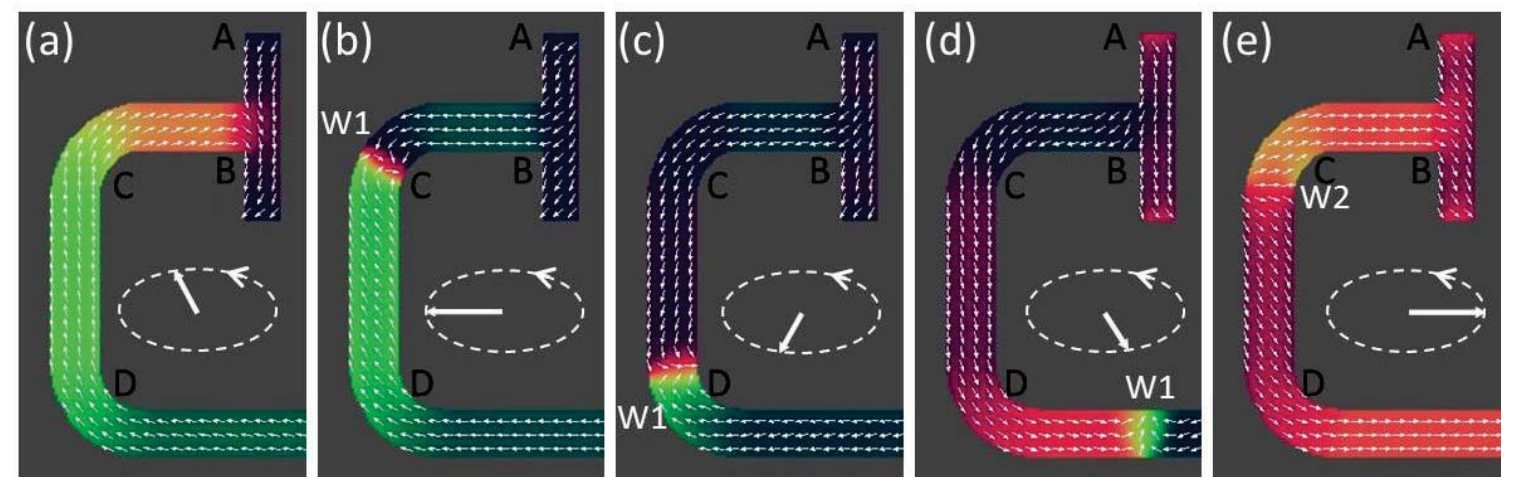

Figure 4.1.2 Simulated magnetic domain structure evolution and unwinding transverse DW generation from the bar-shaped wall generator in one loading cycle of ccw rotating magnetic field with amplitude of $h_{x}=0.06$ and $h_{y}=0.03$ at (a) $\mathrm{t}^{*}=1 / 3$, (b) $\mathrm{t}^{*}=1 / 2$, (c) $\mathrm{t}^{*}=2 / 3$, (d) $\mathrm{t}^{*}=5 / 6$, and (e) $t^{*}=1$. The white solid arrow indicates the elliptical rotating field direction. A labels the bar, B labels the junction, and C-D label the nanowire corners. W1 and W2 are the two unwinding transverse DWs, ccw-hh DW and cw-tt DW, respectively.

Although the bar-shaped data generator producing unwinding transverse DW pairs has been proposed above, the bit definition for generator is still inconsistent with that for logic gates, and then new bit definition applied for both generator and logic gates is needed. Considering there are two types of transverse DW pairs existed, one is the ccw-hh and cw-tt DW pair and the other is the cw-hh and ccw-tt DW pair, new bit 
definition based on those two types of DW pairs can be applied, i.e., the pair of ccw-hh and $\mathrm{cW}-\mathrm{tt}$ DW is defined as ' 1 '(DW magnetization points 'up') while the other pair of cw-hh and ccw-tt DW is defined as ' 0 ' (DW magnetization points 'down'), arbitrarily, as shown in Tab. 4.1 As is discussed later, such a new definition can be applied for both data generator and logic gates.

To generate ' 0 ', i.e., the cw-hh and ccw-tt transverse DW pair, without reversing the magnetization direction of the bar, the bar-shaped wall generator should be modified. Our simulations show that a 'horn' associated bar-shaped wall generator, shown in Tab. I with two types of 'horns' (triangle shape and square shape), can produce '0' DW pairs. Fig. 4.1.3 shows the generation process of a cw-hh and ccw-tt transverse DW pair from a square shape 'horn' in one cycle of ccw rotating field with amplitude of $h_{x}=0.066$ and $h_{y}=0.04$. In the first half cycle (Fig. 4.1.3 (a)-(c)), magnetizations within 'horn' C prefer to follow the rotating field due to its relatively larger region (Fig. 4.1.3 (b)), and eventually produces a cw-hh W1 pushed forward to corner D and a ccw-tt W2 pushed backward to junction B after the first half rotating cycle (Fig. 4.1 .3 (c)). In the second half cycle (Fig. 4.1.3 (d)-(f)), W1 is transported around the nanowire loop, and W2 is also driven by the rotating field to pass through 'horn' C (Fig. 4.1.3 (e)) and arrive at corner D (Fig. 4.1.3 (f)) at the end. Our simulated results show that the critical field to generate ' 1 ' or ' 0 ' strongly depends on the 'horn' shape and its size, which can also be noted from Fig. 4.1.7-4.1.10 for triangle-shaped 'horns' of different sizes as discussed later. Thus, two types of transverse DW pairs, presented as ' 1 ' and ' 0 ' respectively, can be generated by a 'horn' associated bar-shaped wall generator under ccw rotating magnetic field. 
Table 4.1 Schematic drawing of nanometer transverse DW logic elements, including the new bit definition of ' 1 ' and ' 0 ', the bar-shaped data generator associated with a triangle or square shape 'horn', the T/2 delay or no-delay NOT gate, the AND gate, and the OR gate.

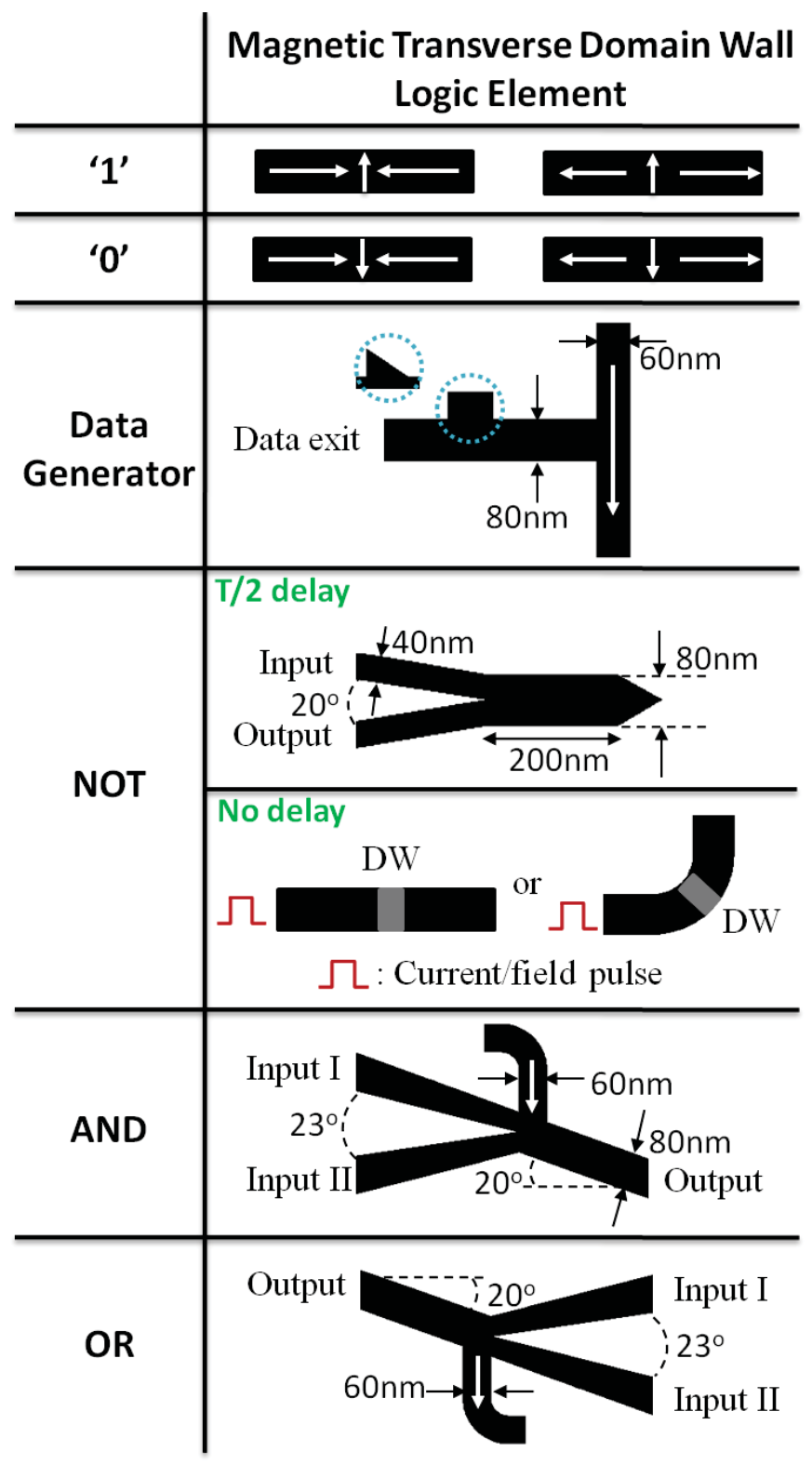



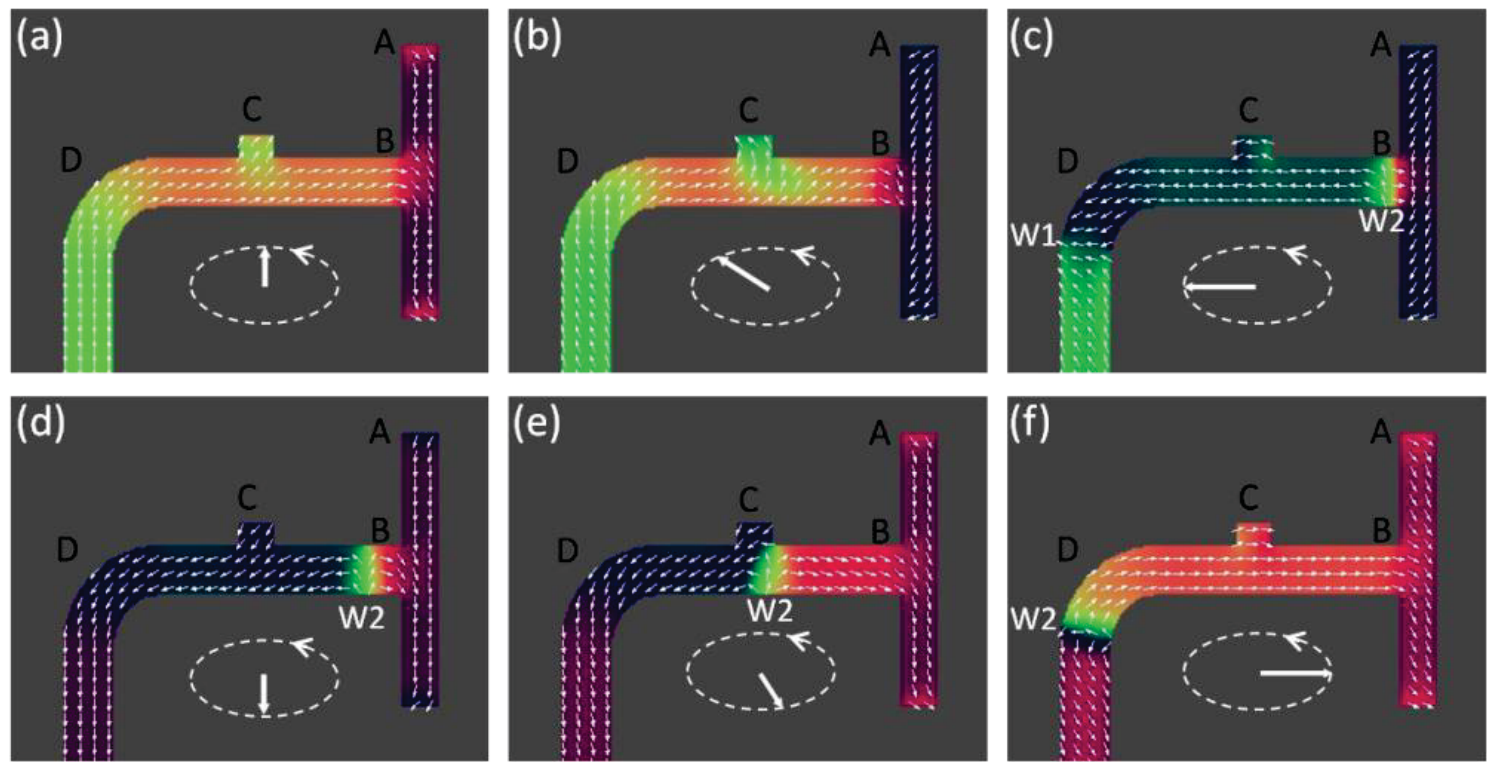

Figure 4.1.3 Simulated magnetic domain structure evolution and unwinding transverse DW generation from the bar-shaped data generator associated with a square shape 'horn' in one loading cycle of ccw rotating magnetic field with amplitude of $h_{x}=0.066$ and $h_{y}=0.04$ at (a) $t^{*}=1 / 4$, (b) $t^{*}=5 / 12$, (c) $t^{*}=1 / 2$, (d) $t^{*}=3 / 4$, (e) $t^{*}=5 / 6$, and (f) $t^{*}=1$. A labels the bar, B labels the junction, $\mathrm{C}$ labels the square shape 'horn', and D labels the nanowire corner. W1 and W2 are the two unwinding transverse DWs, cw-hh DW and ccw-tt DW, respectively.

\subsubsection{NOT}

Based on our new definition for information bits, the cusp-shaped NOT gate can also be used to switch between the two types of DW pairs. Fig. 4.1.4 shows '0' DW pair is transformed into ' 1 ' DW pair by passing through the NOT gate after one cycle (if counted starting from $t^{*}=1 / 2$ ). In the first half cycle (Fig. 4.1 .4 (a)-(d)), the cw-hh DW W1 is transformed into a cw-tt DW W1', while in the second half cycle (Fig. 4.1.4 (e)(h)), the ccw-tt DW W2 is transformed into a ccw-hh DW W2'. Pinning effect and pinning strength introduced by the junction and corner are different for different DW chiralities, for example, W1 is more easily to be transported through the NOT gate than 
W2, as shown by Fig. 4.1.4. Definitely, W1' and W2' are '1' type DWs, with only the sequence inverted. It is noted that the cusp-shaped NOT gate doesn't change the DW chirality (i.e., $\mathrm{cw}(\mathrm{ccw})$ is still $\mathrm{cw}$ (ccw) after transformation), but only makes a T/2 delay (i.e., hh (tt) is transformed to $\mathrm{tt}(\mathrm{hh})$ ). Like the submicrometer NOT gate $[32,177]$, such a T/2 delay makes it difficult to be directly integrated with other logic circuits like AND or OR. In order to avoid the T/2 delay, direct DW chirality flipping between 'up' and 'down' is required.
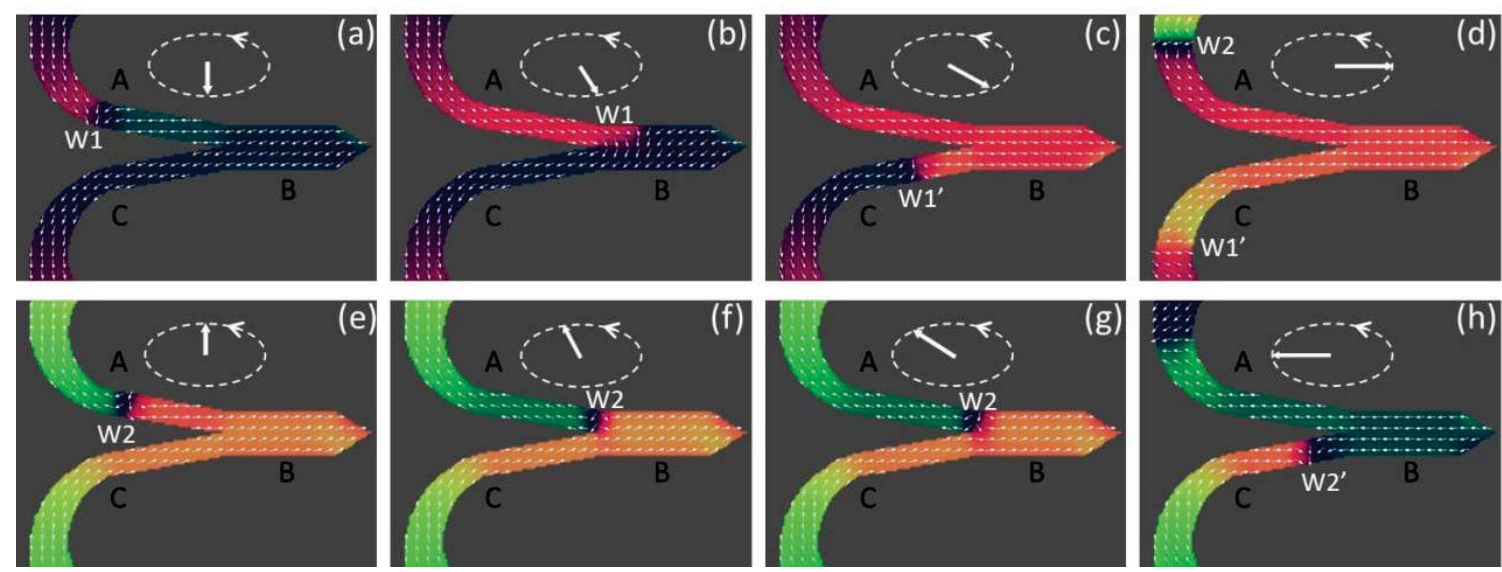

Figure 4.1.4 Simulated magnetic domain structure evolution and unwinding transverse DW pair passing through the NOT gate in 1.5 loading cycles of ccw rotating magnetic field with amplitude of $h_{x}=0.066$ and $h_{y}=0.04$ at (a) $\mathrm{t}^{*}=3 / 4$, (b) $\mathrm{t}^{*}=5 / 6$, (c) $\mathrm{t}^{*}=11 / 12$, (d) $\mathrm{t}^{*}=1$, (e) $\mathrm{t}^{*}=5 / 4$, (f) $t^{*}=4 / 3,(g) t^{*}=17 / 12$, and $(h) t^{*}=3 / 2$. A and $C$ label the input and output branch of NOT gate, respectively, and B labels the NOT gate head. W1 (cw-hh) and W2 (ccw-tt) are the two input unwinding transverse DWs, while W1' (cw-tt) and W2' (ccw-hh) are the corresponding two output unwinding transverse DWs.

As theoretically predicted, periodical chirality flipping associated with velocity oscillation due to the spin precession for transverse DWs can be realized by applying 
external magnetic field or spin-polarized current beyond the Walker threshold. [1, 51] Direct observation for the flipping or oscillation for transverse DWs under magnetic field [16] or spin-polarized current [36] has been reported. Besides, it is observed recently that the chirality of a transverse DW can also be reversed by applying a nanosecond current pulse which induces an Oersted field. [176] The transverse DW flipping realized by the above three methods can be directly used to switch DW pair types between ' 1 ' and ' 0 ' without causing a T/2 delay. Fig. 4.1.5 shows one example based on our micromagnetic simulations, the spin-polarized current induced transverse DW flipping in the nanowire. By applying a large current $\mathrm{u}=2000 \mathrm{~m} / \mathrm{s}$ well above the Walker field, an antivortex with large out-of-plane magnetization component at the core begins formed from the bottom of the transverse DW (Fig. 4.1.5 (b)), and then moves continuously to the up (Fig. 4.1.5 (c)-(d)), eventually eliminated at the nanowire edge associated with strong spin wave propagation caused by the annihilation of out-of-plane magnetization at the core (Fig. 4.1.5 (e)), leaving a reversed transverse DW after 1.15ns.

The proposed NOT gate without T/2 delay requires the current or field pulse to be applied to the nanowire circuit, either at the straight conduit or at the loop corner, as shown in Tab. 4.1 If applied to the loop corner, the threshold values for DWs with different chiralities are also different due to the different pinning effects and pinning strengths, but the DW is localized at the corner, which makes the current or field pulse applied easily. 


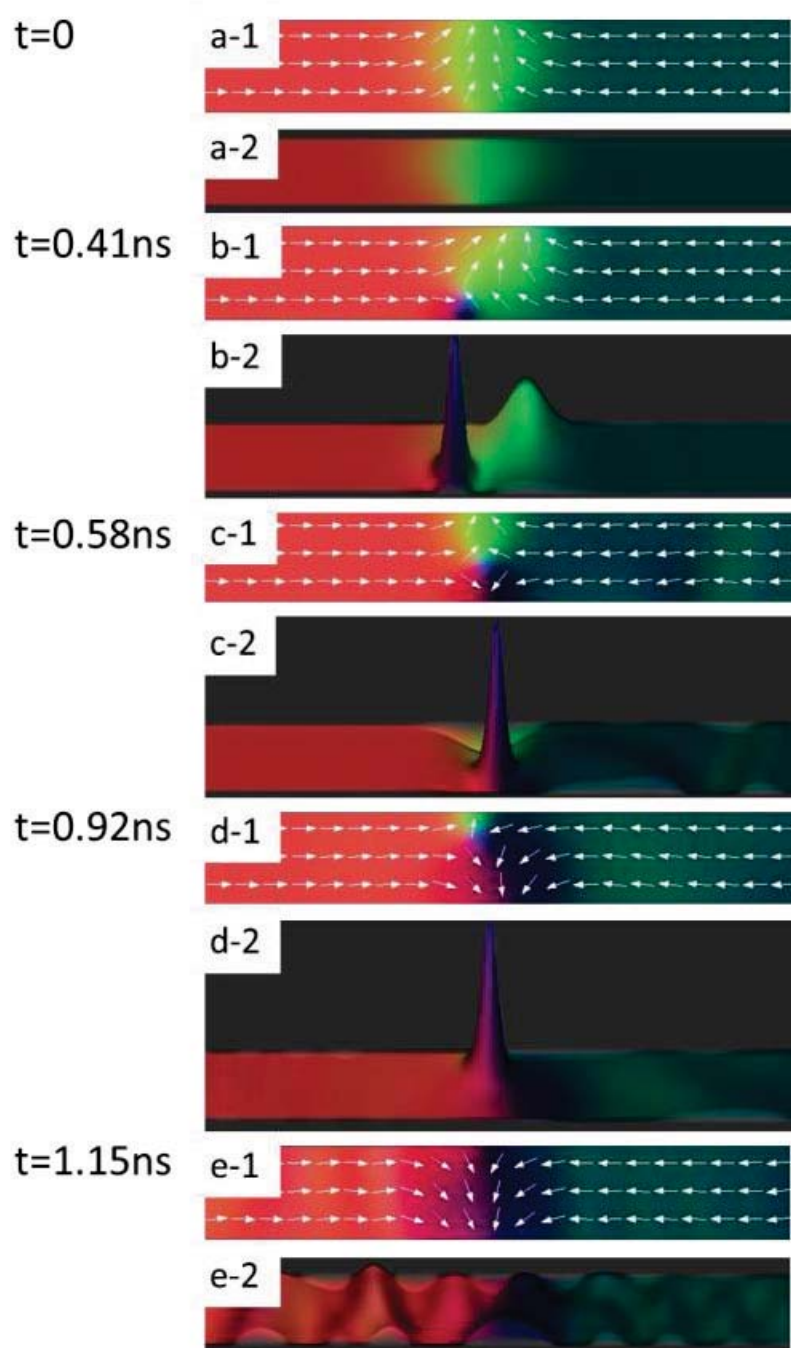

Figure 4.1.5 Simulated spin-polarized current induced transverse DW flipping process by applying a current of $\mathrm{u}=2000 \mathrm{~m} / \mathrm{s}$ at (a) $\mathrm{t}=0 \mathrm{~ns}$, (b) $\mathrm{t}=0.41 \mathrm{~ns}$, (c) $\mathrm{t}=0.58 \mathrm{~ns}$, (d) $\mathrm{t}=0.92 \mathrm{~ns}$, and (e) $\mathrm{t}=1.15 \mathrm{~ns}$. The out-of-plane magnetization component is plot using $3 \mathrm{D}$ visualization shown by (a2)-(e-2).

\subsubsection{AND/OR}

For the AND gate, two input branches carrying transverse DW pairs will be joint for just one output. Since the ' 1 ' and ' 0 ' DWs are equivalent, direct use of the ' $Y$ '-shaped AND gate [34] can't function AND when ' 1 ' and ' 0 ' meet. Therefore, a bar with 
magnetization pointing 'down' is added to the ' $\mathrm{Y}$ '-shaped gate to break the equivalence. Actually, this 'down'-pointed bar performs a '0'-bias to the input DWs, implementing the novel AND gate shown in Tab. 4.1 The left-pointing corner at the top end of the bar actually prevents the reversal of magnetization within the bar under the rotating field. A test circuit is designed for the AND gate by using two data generators associated with two different sizes of triangle shape 'horns' to write data ' 11 ', ' 00 ', ' 01 ' and ' 10 ' as the inputs of AND gate. Fig. 4.1.6 shows the resetting process for the initial test circuit state. At first, a saturation field along $[1 \overline{1} 0]$ indicated by the white arrow is applied to the test circuit and then removed, and the relaxed magnetic domain structure is shown by Fig. 4.1.6 (a). It is noted that the magnetization for bar A1 and A2 points 'down' as expected, but two additional DWs stay at corner $\mathrm{C} 1$ and $\mathrm{C} 2$. In order to clear the two unwanted DWs, a half cycle of ccw rotating field is applied (Fig. 4.1.6 (b)-(c)) to transport them out of the circuit, but the magnetization within bar F is reversed shown in Fig. 4.1.6 (c). To reverse back the magnetization of bar F without creating unwanted DWs, a moderate field along $[00 \overline{1}]$ is applied, and finally, the reset process is finished by making magnetizations of bar A1, A2 and F point 'down', as shown in Fig. 4.1.6 (d).

For the test of ' 1 ' AND ' 1 ' $\rightarrow$ ' 1 ', ccw rotating field with amplitude of $h_{x}=0.054$ and $h_{y}=0.04$ is applied to generate ' 1 ' DW pairs from both data generators, as shown by Fig. 4.1.7. At first, the ccw-hh DWs W1-1 and W2-1 are generated from bar A1 and A2, as shown in Fig. 4.1.7 (b). They are transported around the loop to branch D1 and D2 of the AND gate (Fig. 4.1 .7 (c)) and meet at the junction E to merge into a whole ccw-hh DW which will be transported through $\mathrm{E}$ and out of $\mathrm{G}$ at the end, as shown in Fig. 4.1.7 
(d)-(e). After that, the cw-tt DWs W1-2 and W2-2 (Fig. 4.1 .7 (e)) are also generated and brought to D1 and D2 (Fig. 4.1 .7 (f)) to meet and merge into a whole cw-tt DW (Fig. 4.1.7 (g)) transported through the AND gate at the end (Fig. 4.1.7 (h)). Finally, the process for ' 1 ' AND ' 1 ' $\rightarrow$ ' 1 ' is confirmed for the novel AND gate based on our simulation results. Since the bar F is ' 0 '-bias, magnetization of $F$ is partially reversed after the first DW transported through E, but further reversing is prevented by the pinning effect of the left-pointing corner at the top end. It is noted that the ' 0 ' and ' 1 ' DWs are generated from B1 and A2, respectively, by applying a rotating field with amplitude of $h_{x}=0.064$ and $h_{y}=0.04$ in the last half cycle (Fig. $\left.4.1 .7(\mathrm{e})-(\mathrm{h})\right)$ to prepare input DWs for the process of ' 0 ' AND ' 1 ' $\rightarrow$ ' 0 ' discussed later.
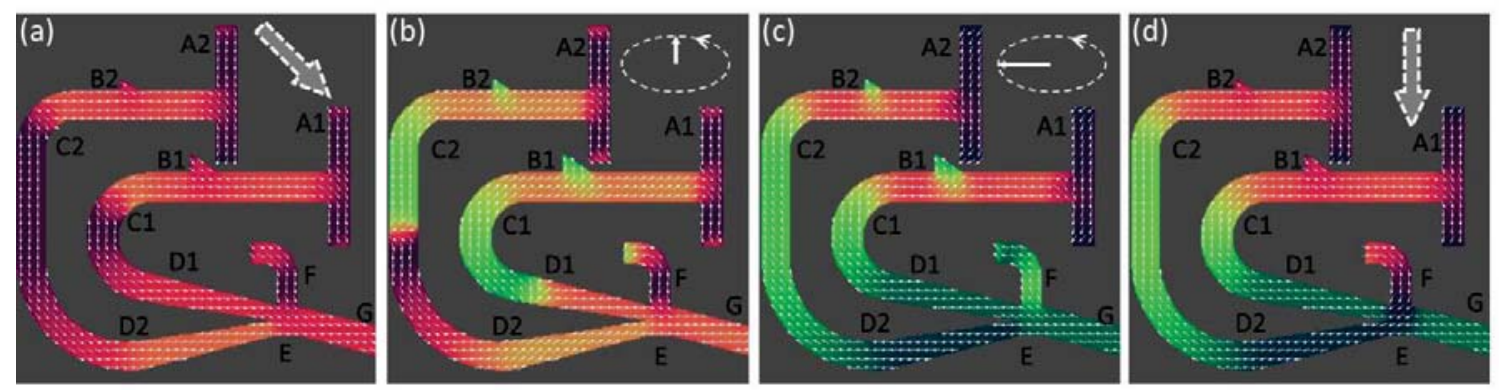

Figure 4.1.6 (a) The relaxed magnetic domain structure upon removal of a saturating magnetic field (white dashed arrow). (b)-(c) Clear-up process in one half loading cycle of ccw rotating magnetic field. (d) The relaxed magnetic domain structure upon removal of a magnetic field [0.0, -0.065] (white dashed arrow). A1 (A2), B1 (B2) and C1 (C2) label the bar, 'horn' and corner of the first (second) data generator, respectively. D1 and D2 label the two input branches, E labels the junction, F labels the ' 0 '-bias bar, and G labels the output of the AND gate. 

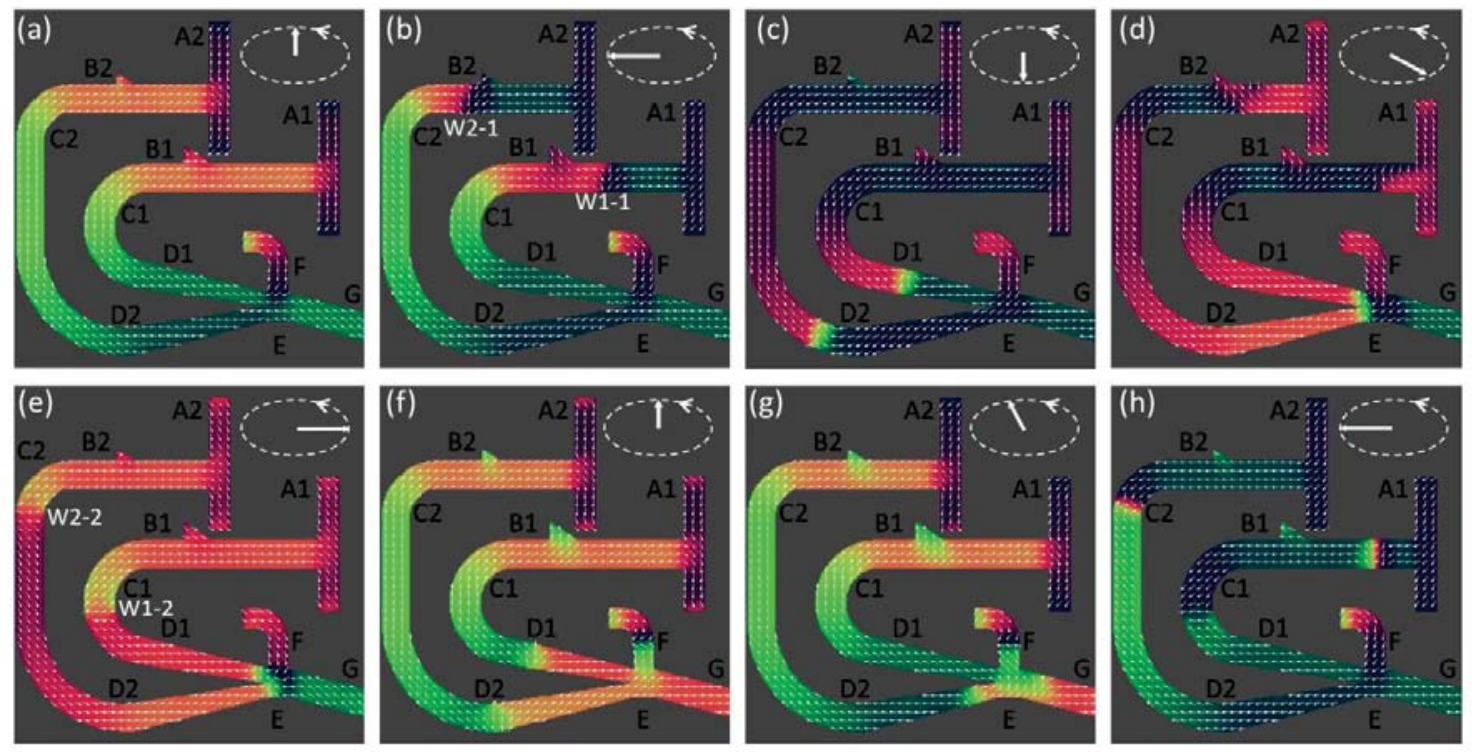

Figure 4.1.7 Simulated process for ' 1 ' AND ' 1 ' $\rightarrow$ ' 1 ' in 1.5 loading cycles of ccw rotating magnetic field at (a) $t^{*}=1 / 4$, (b) $t^{*}=1 / 2$, (c) $t^{*}=3 / 4$, (d) $t^{*}=8 / 9$, (e) $t^{*}=1$, (f) $t^{*}=5 / 4$, (g) $t^{*}=47 / 36$, and (h) $t^{*}=3 / 2$. W1-1 and W1-2 are the ' 1 ' DW pair generated from the first generator, while W21 and W2-2 are the ' 1 ' DW pair generated from the second generator.

The process for ' 0 ' AND ' 0 ' $\rightarrow$ ' 0 ', as shown by Fig. 4.1.8, is similar with that of ' 1 ' AND ' 1 ' $\rightarrow$ ' 1 '. Under rotating field with amplitude of $h_{x}=0.07$ and $h_{y}=0.04$, ' 0 ' DW pair, cw-hh W1-1 (W2-1) and ccw-tt W1-2 (W2-2), is generated from the triangle 'horn’ B1 (B2), as shown by Fig. 4.1.8 (b). W1-1 (W1-2) and W2-1 (W2-2) are driven by the rotating field to meet at the junction $\mathrm{E}$ and merge into a whole $\mathrm{cw}-\mathrm{hh}$ (ccw-tt) DW to pass through E, as shown by Fig. 4.1 .8 (d) (Fig. 4.1 .8 (g)). Unlike the process for ' 1 ' AND ' 1 ' $\rightarrow$ ' 1 ', the ' 0 '-bias bar $\mathrm{F}$ is not partially reversed. Rotating field with amplitude of $h_{x}=0.07$ and $h_{y}=0.033$ is applied in the last half cycle (Fig. $\left.4.1 .8(\mathrm{e})-(\mathrm{h})\right)$ to prepare input DWs for the process of ' 1 ' AND ' 0 ' $\rightarrow$ ' 0 ' discussed later. 

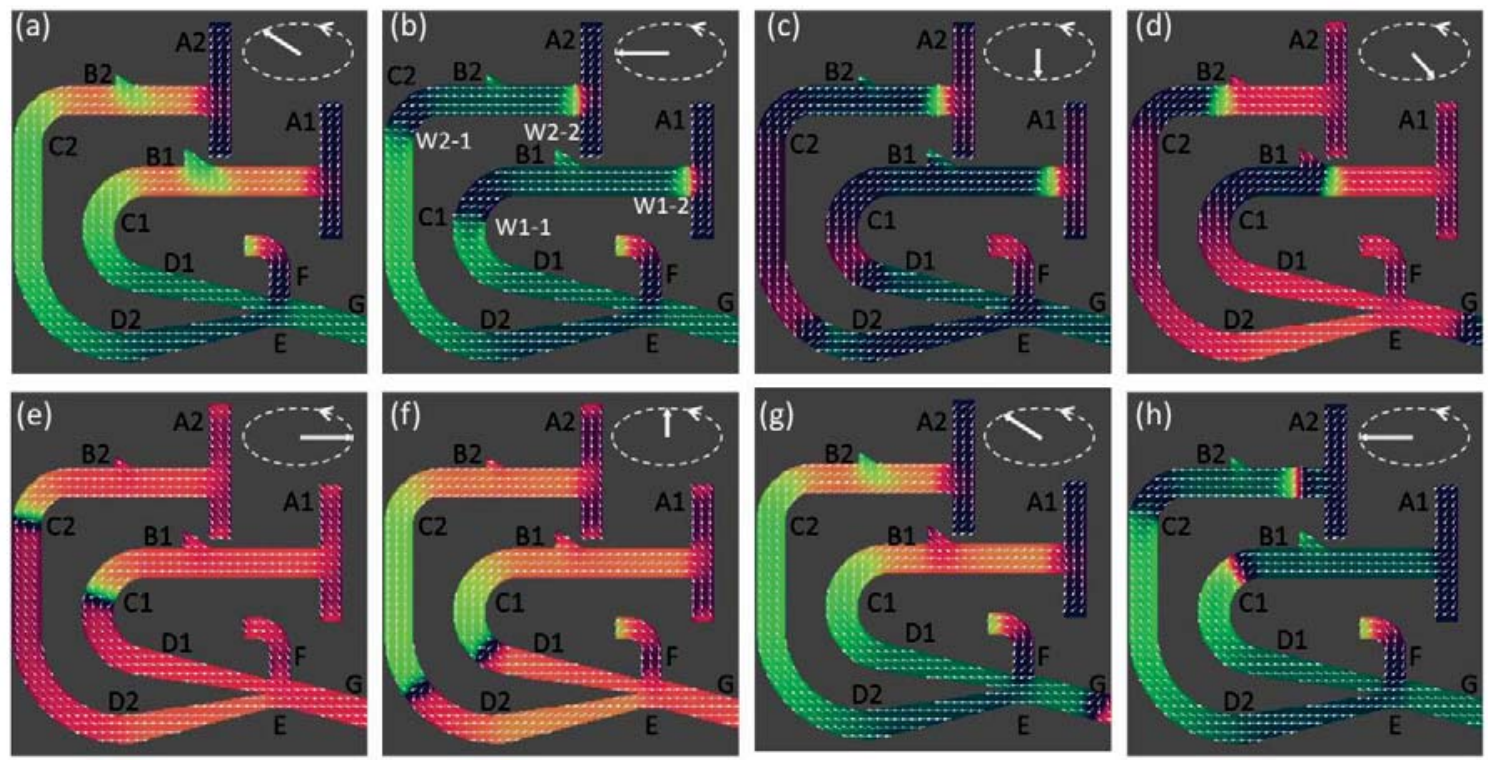

Figure 4.1.8 Simulated process for ' 0 ' AND ' 0 ' $\rightarrow$ ' 0 ' in 1.5 loading cycles of ccw rotating magnetic field at (a) $t^{*}=5 / 12$, (b) $t^{*}=1 / 2$, (c) $t^{*}=3 / 4$, (d) $t^{*}=31 / 36$, (e) $t^{*}=1$, (f) $t^{*}=5 / 4$, (g) $t^{*}=17 / 12$, and $(h) t^{*}=3 / 2$. W1-1 and W1-2 are the ' 0 ' DW pair generated from the first generator, while W2-1 and W2-2 are the '0' DW pair generated from the second generator.

For the process of ' 0 ' AND ' 1 ' $\rightarrow$ ' 0 ', as shown in Fig. 4.1.9, the generation of input DWs are shown in Fig. 4.1.7 (e)-(h). W1-1 and W2-1 are transported to D1 and D2 (Fig. 4.1.9 (a)), and then W1-1 passes through the junction E due to the '0'-bias of bar F (Fig. 4.1 .9 (b)), leaving W2-1 pinned at the end of branch D2 by forming a $360^{\circ} \mathrm{DW}$ (Fig. 4.1.9 (c)). After the second DWs W1-2 and W2-2 enter D1 and D2 (Fig. 4.1.9 (d)), W2-2 meets the previously pinned W2-1 and annihilates each other (Fig. 4.1.9 (e)), while W1-2 passes through the junction E (Fig. 4.1.9 (f)). The whole process indicates that the '0' DW pair can transport through AND gate due to the '0'-bias nature of bar F while the ' 1 ' DW pair will be pinned and finally annihilated in one cycle, confirming the function of ' 0 ' AND ' 1 ' $\rightarrow$ ' 0 '. 

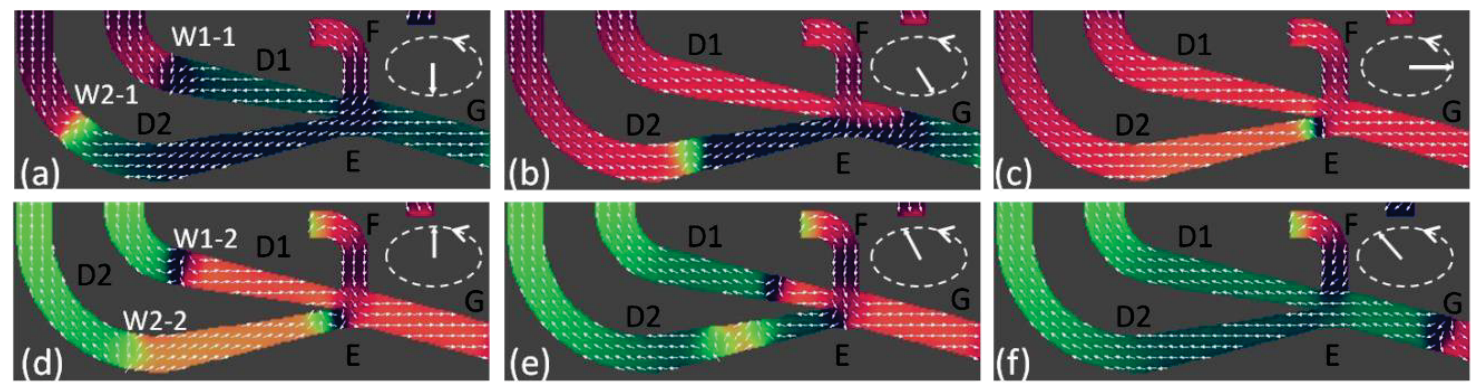

Figure 4.1.9 Simulated process for ' 0 ' AND ' 1 ' $\rightarrow$ ' 0 ' in 1.5 loading cycles of ccw rotating magnetic field at (a) $t^{*}=3 / 4$, (b) $t^{*}=5 / 6$, (c) $t^{*}=1$, (d) $t^{*}=5 / 4$, (e) $t^{*}=47 / 36$, and (f) $t^{*}=49 / 36$. W $1-1$ and W1-2 are the '0' DW pair, while W2-1 and W2-2 are the ' 1 ' DW pair.
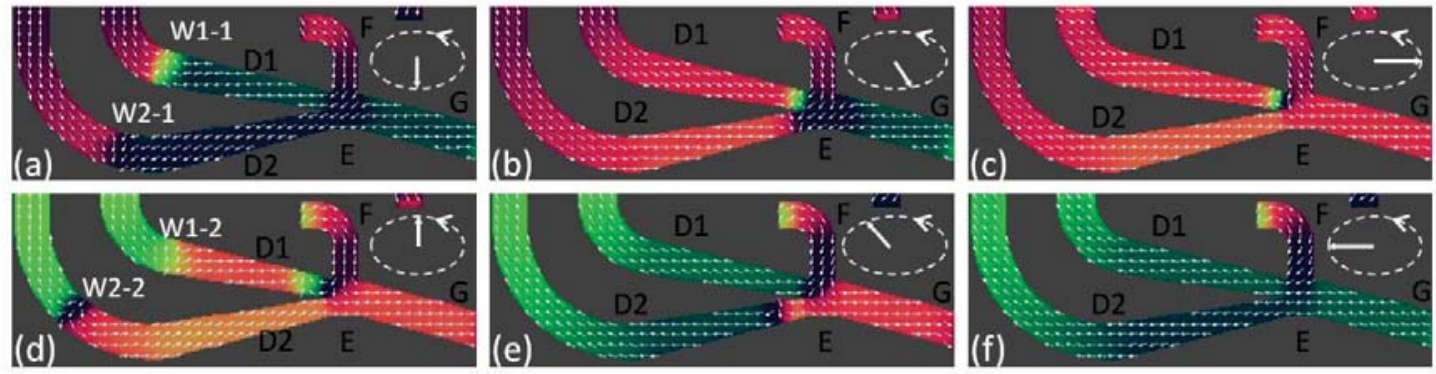

Figure 4.1.10 Simulated process for ' 1 ' AND ' 0 ' $\rightarrow$ ' 0 ' in 1.5 loading cycles of ccw rotating magnetic field at (a) $t^{*}=3 / 4$, (b) $t^{*}=5 / 6$, (c) $t^{*}=1$, (d) $t^{*}=5 / 4$, (e) $t^{*}=49 / 36$, and (f) $t^{*}=3 / 2$. W $1-1$ and W1-2 are the ' 1 ' DW pair, while W2-1 and W2-2 are the ' 0 ' DW pair.

For the process of ' 1 ' AND ' 0 ' $\rightarrow$ ' 0 ', as shown in Fig. 4.1.10, the generation of input DWs are shown by Fig. 4.1.8 (e)-(h). Similarly, the '0' DW pair, W2-1 and W2-2, can transport through the AND gate due to the '0'-bias bar F while the '1' DW pair, W11 and W1-2, will be pinned and finally annihilated in one cycle, confirming the function of ' 1 ' AND ' 0 ' $\rightarrow$ ' 0 '

Thus, the function of AND has been confirmed by the above four simulation cases shown by Fig. 4.1.7-4.1.10, where the '0'-bias bar F plays an important role. Since the magnetization within the '0'-bias bar can be partially reversed, as shown by Fig. 4.1.7 (f), 106 
and may be even totally reversed by larger field, in that case, the ' 0 '-bias is not guaranteed and hence the AND function will be influenced. To absolutely avoid the totally reversal under rotating field as to ensure the ' 0 '-bias, an ' $S$ '-shaped bar can be introduced. Fig. 4.1.11 (a) shows the steadied AND gate associated with an 'S'-shaped '0'-bias bar. Fig. 4.1.11 (b)-(e) show the DW propagation around the 'S'-shaped bar under one cycle of ccw rotating field. It is noted that two corners, A and B, with different conduct directions, effectively prevent the DW transported out through B under rotating field. Therefore, the steadied AND gate associated with an ' $S$ '-shaped bar will absolutely avoid the totally reversal of magnetizations within the bar and hence the ' 0 '-bias will be always ensured.

The OR gate shares the same design with AND gate, only with a $180^{\circ}$ angle rotation, as shown in Tab.4.1 Therefore, there is always a $\pi / 2$ phase difference between OR and AND gates. For the OR gate, the magnetization within the bar should still point 'down' to ensure ' 1 '-bias instead, which can be realized in the initial reset process the same with AND gate shown by Fig. 4.1.6. As is expected, the four processes for ' 0 ' OR ' 0 ' $\rightarrow$ ' 0 ', ' 1 ' $\mathrm{OR}$ ' 1 ' $\rightarrow$ ' 1 ', ' 1 ' $\mathrm{OR}$ ' 0 ' $\rightarrow$ ' 1 ', and ' 0 ' $\mathrm{OR}$ ' 1 ' $\rightarrow$ ' 1 ' will be the same with those for AND gate shown in Fig. 4.1.7-4.1.10, respectively. 


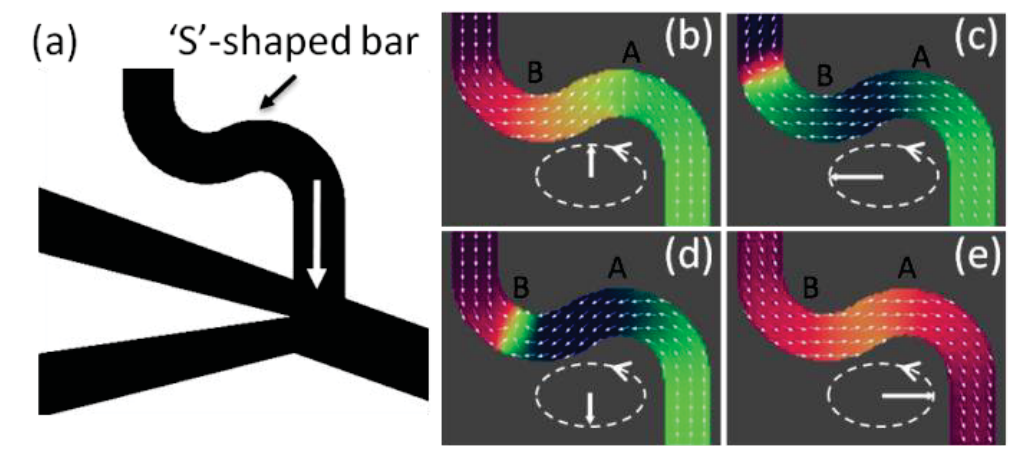

Figure 4.1.11 (a) Schematic drawing of steadied AND gate with ' $S$ '-shaped '0'-bias bar. Simulated transverse DW propagation around the ' $\mathrm{S}$ '-shaped nanowire in one loading cycle of ccw rotating magnetic field at (a) $t^{*}=1 / 4$, (b) $t^{*}=1 / 2$, (c) $t^{*}=3 / 4$, and (d) $t^{*}=1$. A and B label the two round corners.

\subsubsection{Conclusion}

In conclusion, nanometer transverse-DW-based logic devices have been proposed and tested by our micromagnetic simulations. The bar-shaped transverse DW generator which produces unwinding DW pairs under rotating magnetic field is introduced to make the miniaturized submicrometer logic devices [34] feasible in nanowire circuits by avoiding the DW pile-up effect. Adding a triangle or square shape 'horn' to the transverse DW generator, two types of unwinding DW pairs can be generated and hence the information bit ' 1 ' and ' 0 ' can be defined accordingly, i.e., ' 1 ' corresponds to the DW pair with their wall magnetizations point 'up' (or 'left' with respect to the propagation direction) while ' 0 ' corresponds to the DW pair with their wall magnetizations point 'down' (or 'right' with respect to the propagation direction). Based on the new definition, NOT and AND/OR gates are proposed. The miniaturized submicrometer cusp-shaped NOT gate can also perform NOT function for the new bit definition, but there is still a T/2 signal delay which limits its further application. Since magnetic field, spin-polarized 
current as well as current induced Oersted field are observed to be able to flip the chirality of transverse DWs, $[16,36,176]$ the no-delay current/field assistant NOT gate has been proposed, which can be connected to other logic circuits easily without T/2 delay. The AND gate is realized by adding a ' 0 '-bias bar whose magnetization always points 'down' to the ' $\mathrm{Y}$ '-shaped nanowire fork. This ' 0 '-bias bar prefers ' 0 ' DW pair to transport but prevents ' 1 ' DW pair unless two ' 1 ' DW pairs in both branches meet and transport together, leading to the AND function confirmed by our simulated results. The OR gate is easily implemented just by rotating $180^{\circ}$ angle of AND gate, i.e., a $\pi / 2$ phase difference between AND and OR exists, but the magnetization with the bar should still point 'down' to perform a ' 1 '-bias instead. The same bit definition is used for our nanometer data generator and logic gates including NOT and AND/OR, unlike the submicrometer DW logic [34] for which different bit definitions are used for data generator and logic gates. Thus, the nanometer magnetic transverse-DW-based logic elements present more advantages than the submicrometer DW logic, and provide an alternate route to traditional electronics logic. 


\subsection{Generator and Shift Register Based on $360^{\circ}$ Domain Walls}

\subsubsection{Introduction}

$360^{\circ}$ domain walls are common in magnetic thin films and were observed as early as in 1960s in both single- and multi-layer films. [181-183] $360^{\circ}$ domain walls were considered troublesome in magnetic films because they significantly influence magnetization processes while are difficult to remove due to their stability. [184-187] However, the usually unwanted $360^{\circ}$ domain walls in magnetic films recently attract attentions for their potential new functionalities in miniaturized devices, for example, sensor application in patterned magnetic films, [188] magnetic random access memory cell in nanorings, $[189,190]$ and magnetic memory and logic device in multilayer stripes. [191] For the applications of $360^{\circ}$ domain walls, reliable production and accurate control of them are necessary. This paper presents a micromagnetic simulation study of $360^{\circ}$ domain wall behaviors in planar nanowire loops. In particular, it is shown that a nanowire loop with a shape-isotropic (round-shaped) wall generator at one end and a shapeanisotropic (bar-shaped) wall stopper at the other end functions like a data storage stack: $360^{\circ}$ domain walls are generated and pushed into stack under rotating field before overflow, while popped out and annihilated when field rotating direction is inverted until underflow. The stack capacity is determined by the total length of the nanowire loop.

A $360^{\circ}$ domain wall generator was previously designed by spin valve patterning, which is composed of a circle or octagon-shaped generator, a wedge-shaped stripe, and a long and thin stripe with fixed magnetization. [188] The generation of one $360^{\circ}$ domain wall is demonstrated, while to produce more walls, a shielding layer is required for some 
part of the generator, which limits its applications. [188] In this section, a simple wall generator and storage stack is designed in the form of a nanowire loop with a shapeisotropic (round or hexagonal) wall generator at one end and a shape-anisotropic (barshaped) wall stopper at the other end as shown in Fig. 4.2.1, which can generate, store and annihilate multiple $360^{\circ}$ domain walls simply via in-plane rotating magnetic field. The round-shaped generator serves as a nucleation site for domain walls under rotating field due to its shape isotropy, while the bar-shaped stopper functions as a barrier to domain walls due to its shape anisotropy. Such a nanowire loop generates one $360^{\circ}$ domain wall per loading cycle of the counterclockwise-rotating magnetic field; the generated $360^{\circ}$ domain walls are pushed into the nanowire loop and stored together, coexisting stably; the stored $360^{\circ}$ domain walls can be annihilated one by one simply by inverting the magnetic field rotation direction to clockwise. The simulation results of detailed $360^{\circ}$ domain wall behaviors in the nanowire are discussed in next section. Besides, enlightened by the design of $360^{\circ}$ domain wall generator operated by continuous rotating field, a shift register based on the presence and absence of a $360^{\circ}$ domain wall in a winding planar nanowire operated by more complicated rotating field paths is proposed, which will be discussed in Sec. 4.2.3. All the simulation details for the two proposed devices can be found in Sec. 4.1.

\subsubsection{0 ${ }^{\circ}$ Domain Wall Generator}

The simulation starts with the magnetic domain structure in Fig. 4.2.1 (b), which is the relaxed state after removal of a saturating magnetic field $\mathbf{H} / M_{\mathrm{s}}=(0.15,-0.15)$ as 
shown by the white dashed arrow. As expected, the magnetization vectors in the nanowire loop align along the length direction of the wire sections complying with the shape anisotropy, pointing to right in horizontal sections and down in vertical sections as well as in the wall stopper $\mathrm{J}$, as preferred by the pre-applied saturating magnetic field. As a result, four $180^{\circ}$ Néel walls are formed respectively at corners $\mathrm{B}, \mathrm{D}, \mathrm{F}$ and $\mathrm{H}$. It is noted that these $180^{\circ}$ domain walls in the nanowire loop fall into two types based on magnetization vector direction in the wall center: pointing inward towards the loop center (at B and F) or outward (at D and H), which are respectively of tail-to-tail and head-tohead configuration; for convenience the former will be called (-) type while the latter (+) type It is also noted that two $180^{\circ}$ domain walls of different types combine to form one $360^{\circ}$ domain wall. Therefore, to form a $360^{\circ}$ domain wall, two $180^{\circ}$ domain walls of different types must be generated in sequence and subsequently brought into contact, as the designed unit in Fig. 4.2.1(a) does under rotating field. Now an elliptical counterclockwise rotating magnetic field $\mathbf{H} / M_{\mathrm{s}}=\left[0.04 \cos \left(2 \pi \mathrm{t}^{*}\right), 0.016 \sin \left(2 \pi \mathrm{t}^{*}\right)\right]$ is applied to the initial domain structure in Fig. 4.2.1(b). For the given design, a counterclockwise rotating field nucleates a pair of $(+)$ and (-) $180^{\circ}$ domain walls per cycle from the hexagonal wall generator $\mathrm{A}$ and transports them away around the bending corner B into the nanowire loop. An elliptical rotating field with a smaller vertical component is used in order to prevent domain switching in the wall stopper $\mathrm{J}$, which ensures to block domain walls arriving along the nanowire section IJ. For clarity, the domain walls generated during the $n$th loading cycle will be called $(n+)$ and $(n-)$ with $+/-$ 
signs indicating the wall types. The four pre-existing domain walls at corners B, D, F and $\mathrm{H}$ are therefore $(0-),(0+),(0-)$ and $(0+)$, respectively.
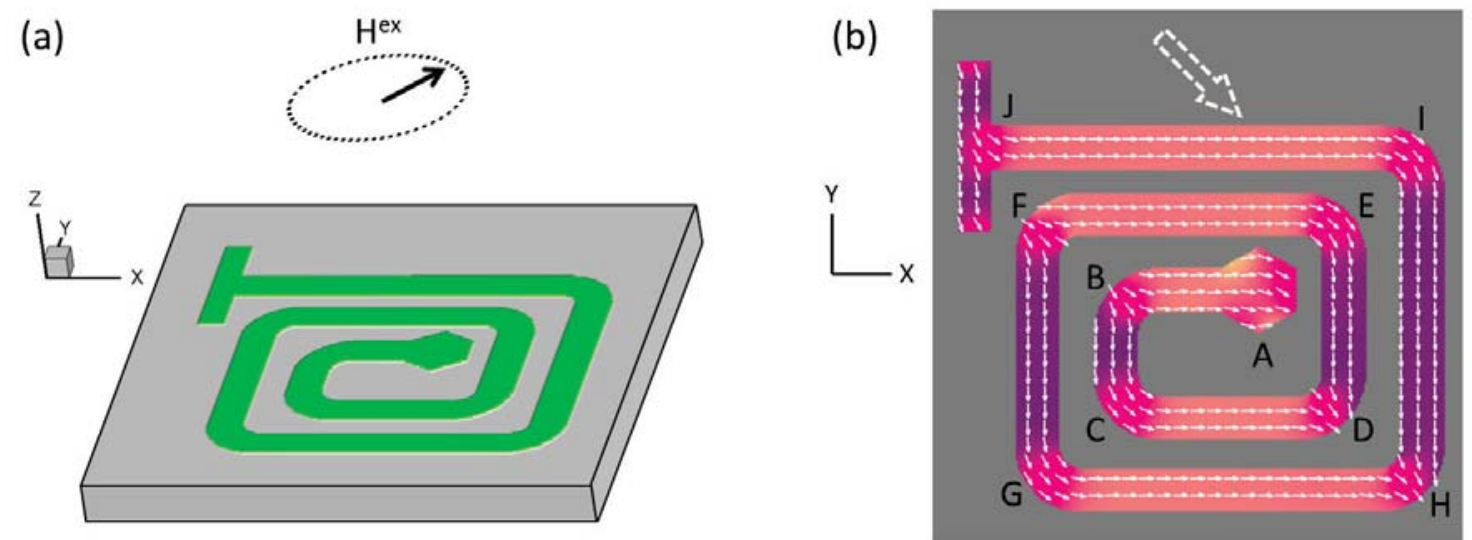

Figure 4.2.1 (a) Planar nanowire loop with a hexagonal wall generator and a bar-shaped wall stopper, as patterned from a magnetic thin film on nonmagnetic substrate, under in-plane rotating magnetic field $\mathbf{H}^{\mathrm{ex}}$. (b) The relaxed magnetic domain structure upon removal of a saturating magnetic field (white dashed arrow). Small white arrows and color contours within the nanowire represent magnetization direction. A and $\mathrm{J}$ label the wall generator and stopper, respectively, and B-I label the eight bending corners of the nanowire in the order from the wall generator to the wall stopper.

Fig. 4.2.2 shows detailed magnetization process during the first two loading cycles $\left(t^{*}=0-2\right)$, generating two $360^{\circ}$ domain walls that are pushed into the nanowire and stored there. Consider the first loading cycle $\left(t^{*}=0-1\right)$ in Fig. 4.2.2(a)-(d). In the first quarter of the loading cycle $\left(\mathrm{t}^{*}=0-0.25\right)$ in Fig. 4.2.1(b) and Fig. 4.2.2(a), the four preexisting $180^{\circ}$ domain walls at $\mathrm{B}, \mathrm{D}, \mathrm{F}$ and $\mathrm{H}$ move counterclockwise to the next corners C, E, G and I. In the second quarter of the loading cycle $\left(t^{*}=0.25-0.5\right)$ in Fig. 4.2.2(a) and (b), in addition to the further counterclockwise transportation of the four pre-existing 
$180^{\circ}$ domain walls to the next corners $\mathrm{D}, \mathrm{F}, \mathrm{H}$ and the wall stopper $\mathrm{J}$, a new $180^{\circ}$ domain wall (1+) is formed by the generator A and pushed to corner B. In the third quarter of the loading cycle $\left(\mathrm{t}^{*}=0.5-0.75\right)$ in Fig. $4.2 .2(\mathrm{~b})$ and $(\mathrm{c})$, the existing $180^{\circ}$ domain walls keep moving to the next corners towards the wall stopper J. In the fourth quarter of the loading cycle $\left(t^{*}=0.75-1\right)$ in Fig. 4.2.2(c) and (d), in addition to continuous movement of existing $180^{\circ}$ domain walls to the next corners, a new $180^{\circ}$ domain wall (1-) is formed by the generator A and pushed to corner B. Therefore, during the first loading cycle, a pair of (1+) and (1-) $180^{\circ}$ domain walls are generated, which together with the pre-existing domain walls move in the nanowire loop in the same counterclockwise direction as the rotating field, and the pre-existing (0+) and (0-) $180^{\circ}$ domain walls hit the wall stopper $\mathbf{J}$ in sequence and combine there to form the first $360^{\circ}$ domain wall. As a net result of the first loading cycle, one $360^{\circ}$ domain wall is generated, and the unit is ready to repeat the same operations for the second loading cycle shown in Fig. 4.2.2(e)-(h). It is noted that during the fourth quarter of the loading cycle, while the (0-) domain wall at corner I in Fig. 4.2.2(c) moves counterclockwise towards the wall stopper $\mathrm{J}$, the $(0+)$ domain wall blocked by the wall stopper $\mathbf{J}$ moves clockwise in opposite direction towards corner I, leading to a collision between a pair of $(0+)$ and (0-) domain walls and their combination to form the first $360^{\circ}$ domain wall in the nanowire loop that is stored in section IJ in Fig. 4.2.2(d). The bar-shaped wall stopper $\mathrm{J}$ does not undergo domain switching under the elliptical rotating magnetic field due to its shape anisotropy. It thus stops domain wall motion in the second and third quarters of the loading cycle while repels domain wall in 
the fourth quarter, as shown in Fig. 4.2.2(b)-(d), facilitating $180^{\circ}$ domain wall combination and thus $360^{\circ}$ domain wall formation.
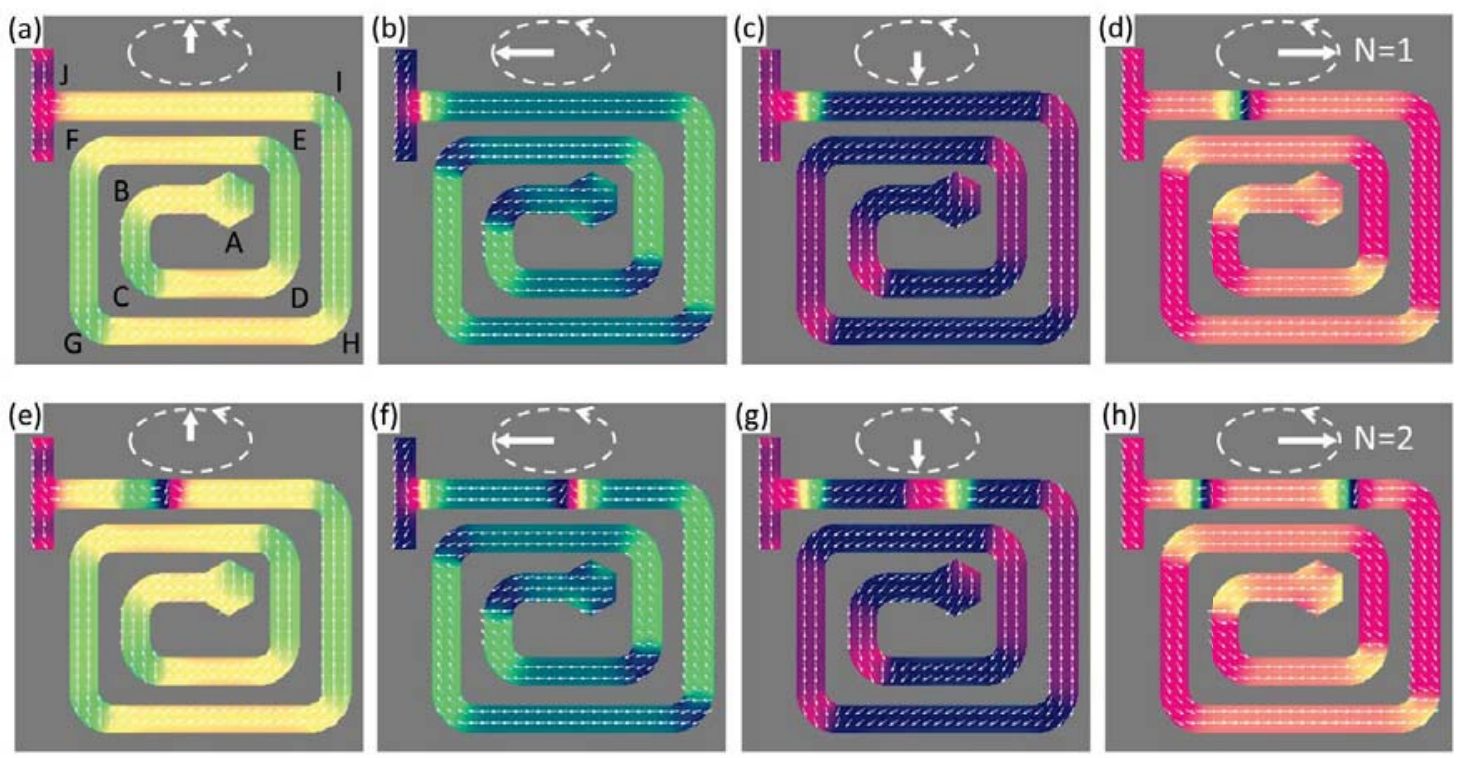

Figure 4.2.2 Simulated magnetic domain structure evolution and domain wall generation for the first two loading cycles of counterclockwise rotating magnetic field at (a) $t^{*}=0.25$, (b) $t^{*}=0.5$, (c) $\mathrm{t}^{*}=0.75$, (d) $\mathrm{t}^{*}=1$, (e) $\mathrm{t}^{*}=1.25$, (f) $\mathrm{t}^{*}=1.5$, (g) $\mathrm{t}^{*}=1.75$, and (h) $\mathrm{t}^{*}=2.0$. The white solid arrow indicates the elliptical rotating field direction. Small white arrows and color contours within the nanowire represent magnetization direction. $\mathrm{N}$ indicates the number of $360^{\circ}$ domain walls stored in the nanowire.

The second loading cycle ( $\left.\mathrm{t}^{*}=1-2\right)$, as shown in Fig. 4.2.2(e)-(h), repeats the same magnetization process as in the first loading cycle $\left(t^{*}=0-1\right)$ : two $180^{\circ}$ domain walls $2(+)$ and 2(-) are generated respectively in the second quarter in Fig. 4.2.2(f) and the fourth quarter in Fig. 4.2.2(h) of the loading cycle, and the second $360^{\circ}$ domain wall is formed in Fig. 4.2.2(h). Such a process can be repeated under continuous operation of rotating magnetic field to form multiple $360^{\circ}$ domain walls. Simulation examples of the generated and stored five, ten and twenty-eight $360^{\circ}$ domain walls are shown in Fig. 4.2.3. It is 
found that one loading cycle generates one new $360^{\circ}$ domain wall if the domain wall density is not high thus the nanowire loop still has free space for a newly formed domain wall to be pushed in; once full, a newly generated domain wall cannot be transported away from the generator A because it is blocked by a full array of domain walls stored in the nanowire loop. Such a situation resembles an overflow status of a data storage stack. The stack capacity is determined by the total length of the nanowire loop. Just like in the above discussed fourth quarter of the loading cycle in Fig. 4.2.2(d), the blocked domain walls would move backwards under the rotating magnetic field towards the generator A thus interfering with the generation of the next domain wall; as a result, formation of a new $360^{\circ}$ domain wall requires increasingly more loading cycles, and finally reaches saturation state shown in Fig. 4.2.3(c), where twenty-eight $360^{\circ}$ domain walls are densely stored in the nanowire loop between the wall generator A and wall stopper J. In addition to an effective generation and storage of $360^{\circ}$ domain walls as demonstrated in Figs. 4.2.2 and 3, annihilation of $360^{\circ}$ domain walls in the nanowire loop can be performed by simply inverting the magnetic field rotation direction to clockwise, as shown in Fig. 4.2.4.
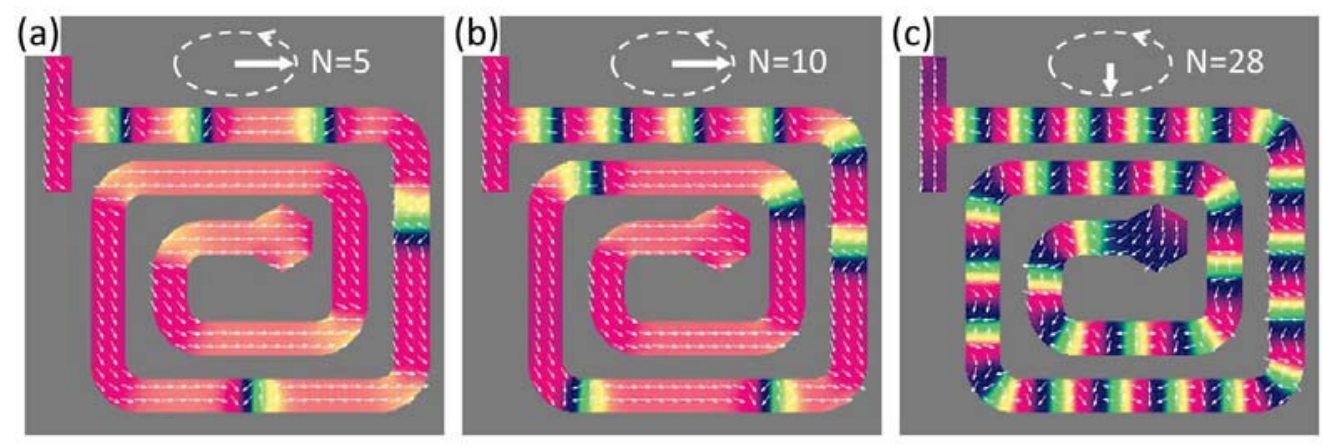

Figure 4.2.3 Simulated generation and storage of multiple $360^{\circ}$ domain walls under multiple cycles of counterclockwise rotating magnetic field at (a) $t^{*}=5$, (b) $t^{*}=10$, and (c) $t^{*}=59.75$. 
By inverting the rotating magnetic field to clockwise direction, $\mathbf{H} / M_{\mathrm{s}}=\left[0.04 \cos \left(2 \pi \mathrm{t}^{*}\right),-0.016 \sin \left(2 \pi \mathrm{t}^{*}\right)\right]$ is now applied to the nanowire loop containing ten $360^{\circ}$ domain walls in Fig. 4.2.3(b). The simulated magnetic domain evolution and domain wall annihilation after the first, fifth and ninth loading cycles are shown in Fig. 4.2.4. Comparing with the initial domain structure in Fig. 4.2.3(b), each loading cycle annihilates one $360^{\circ}$ domain wall from the nanowire loop. Detailed domain evolution reveals that, under clockwise rotating magnetic field, the wall generator A does not generate new domain walls; instead it annihilates the arriving domain walls transported along the nanowire section BA. Unlike counterclockwise rotating field that combines $180^{\circ}$ domain walls to form new $360^{\circ}$ domain walls, clockwise rotating field dissociates $360^{\circ}$ domain walls into $180^{\circ}$ domain walls and releases one $180^{\circ}$ domain wall per half loading cycle (thus a whole pair per loading cycle). The released $180^{\circ}$ domain walls move clockwise towards the wall generator A and are annihilated there. This behavior resembles the pop out of data from a storage stack; domain walls are popped out of the nanowire and annihilated at the wall generator when field rotating direction is inverted until underflow.

Simulation results show that by switching the rotation direction of the magnetic field between counterclockwise and clockwise, generation, storage and annihilation of $360^{\circ}$ domain walls can be easily performed with the designed nanowire loop. Such a unit behaves like a data generator and storage stack. The shape isotropy of the wall generator (round shape) facilitates magnetization rotation to generate new domain walls under counterclockwise rotating field or annihilate existing domain walls under clockwise 
rotating field. The shape anisotropy of the wall stopper (bar shape) prevents domain switching under rotating magnetic field to block domain walls in the nanowire loop. Thus, domain walls are confined within the nanowire loop to participate in the association (combining) and dissociation reactions to form or annihilate $360^{\circ}$ domain walls. The nanowire bending corner $\mathrm{B}$ adjacent to the wall generator A plays an important role in pushing domain walls into the nanowire loop and storing multiple $360^{\circ}$ domain walls, while the maximum number of $360^{\circ}$ domain walls stored in the unit is limited by the total length of the nanowire loop.
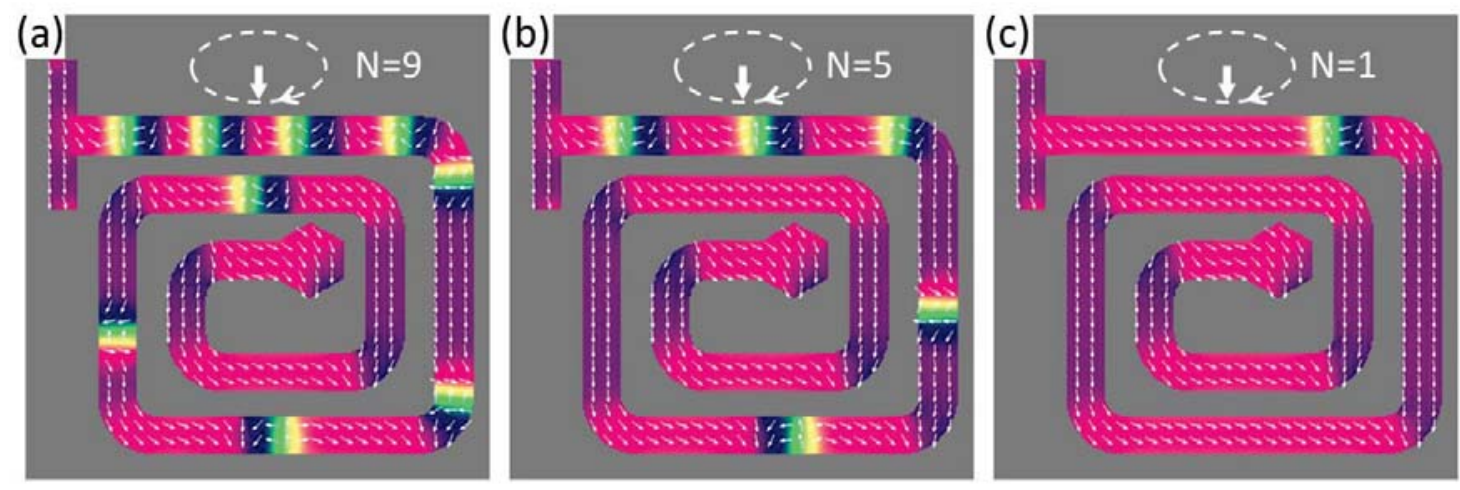

Figure 4.2.4 Simulated annihilation of $360^{\circ}$ domain walls under clockwise rotating magnetic field at (a) $t^{*}=1.25$, (b) $t^{*}=5.25$, and (c) $t^{*}=9.25$.

\subsubsection{0 ${ }^{\circ}$ Domain Wall Shift Register}

As discussed in Sec. $4.2 .2,360^{\circ}$ domain walls can be generated from the isotropic wall generator under counterclockwise rotating magnetic field while eliminated under clockwise field. If the generated $360^{\circ}$ domain walls can be steadily shifted one by one under the external field, the shift register can be therefore constructed. Unfortunately, it is 
even impossible for a $360^{\circ}$ domain wall to move a net distance in a straight nanowire under external magnetic field of any form. However, our simulation results show that the winding nanowire geometry makes the shifting of $360^{\circ}$ domain walls possible. Fig. 4.2.5 (a) shows the nanowire geometry of our $360^{\circ}$ domain wall shift register design which includes an isotropic wall generator followed by a long winding nanowire which will store the $360^{\circ}$ domain walls generated by the wall generator. The information state ' 0 ' and ' 1 ' are defined by the absence and present of a $360^{\circ}$ domain wall stored in the nanowire corner, as shown by Fig. 4.2 .5 (b) and (c), respectively.

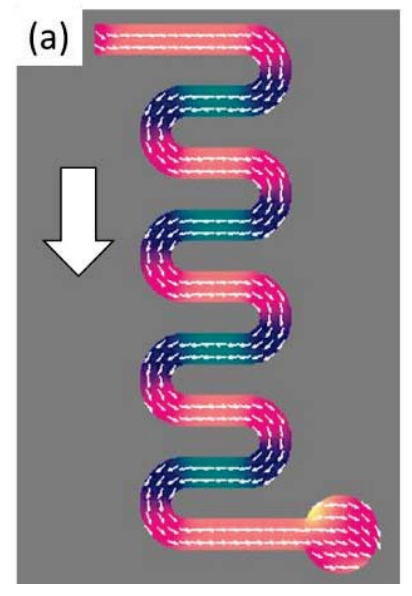

(b)

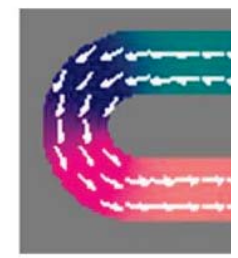

(c)

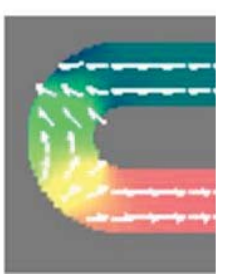

Figure 4.2.5 (a) The relaxed magnetic domain structure upon removal of a saturating magnetic field (white dashed arrow). (b) Definition of state ' 0 ' (without $360^{\circ}$ domain wall) and ' 1 ' (with $360^{\circ}$ domain wall).

The data writing process can be completed by applying the magnetic field in a complicated operation path described in Fig. 4.2.6. It is noted that the operation paths actually include a circular path and an ellipse path, and the only difference between writing ' 0 ' and ' 1 ' is attributed to the circular path, i.e., larger field amplitude is required 
to generate the $360^{\circ}$ domain wall. In spite of writing ' 1 ' or ' 0 ', both operation paths ensure that the stored $360^{\circ}$ domain walls will be shifted to the next corner after one operation cycle.

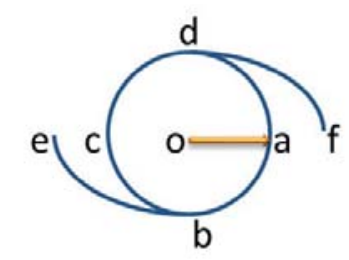

\section{Writing '1': $\mathrm{H}_{\mathrm{oa}}=\mathrm{H}_{\mathrm{ob}}=\mathrm{H}_{\mathrm{oc}}=\mathrm{H}_{\mathrm{od}}=0.019 \mathrm{M}_{\mathrm{s}}, \mathrm{H}_{\mathrm{oe}}=\mathrm{H}_{\mathrm{of}}=0.028 \mathrm{M}_{\mathrm{s}}$ $a-b-c-d-f-d-c-d-a-b-e-b-a \rightarrow$ relax \\ Writing ' 0 ': $\mathrm{H}_{\mathrm{oa}}=\mathrm{H}_{\mathrm{ob}}=\mathrm{H}_{\mathrm{oc}}=\mathrm{H}_{\mathrm{od}}=0.017 \mathrm{M}_{\mathrm{s}}, \mathrm{H}_{\mathrm{oe}}=\mathrm{H}_{\mathrm{of}}=0.028 \mathrm{M}_{\mathrm{s}}$ $a-b-c-d-f-d-c-d-a-b-e-b-a \rightarrow$ relax}

Figure 4.2.6 Applied magnetic field amplitudes and the operation paths to write ' 0 ' and ' 1 '.

Fig. 4.2.7 shows the magnetization process of writing three information bits '1-10 ' to the shift register under three operation cycles of magnetic field applied in a certain path described by Fig. 4.2.6. For the initial empty state shown in Fig. 4.2 .7 (a), it can be realized by initially applying a saturation field pointing downward and then removing it, as shown in Fig. 4.2.5 (a). It is noted that the corner adjacent to the wall generator is not used as information storage.

Besides of the writing process, the erasing process can also be realized by applying the magnetic field according to the path described in Fig. 4.2 .8 (a). For the elimination path, the amplitude is the same with that to write ' 0 ', but the rotating 
direction is quite different. Fig. 4.2 .8 (b) and (c) show the magnetization process of erasing bit ' 0 ' from ' $1-1-0$ ' stored in the shift register.
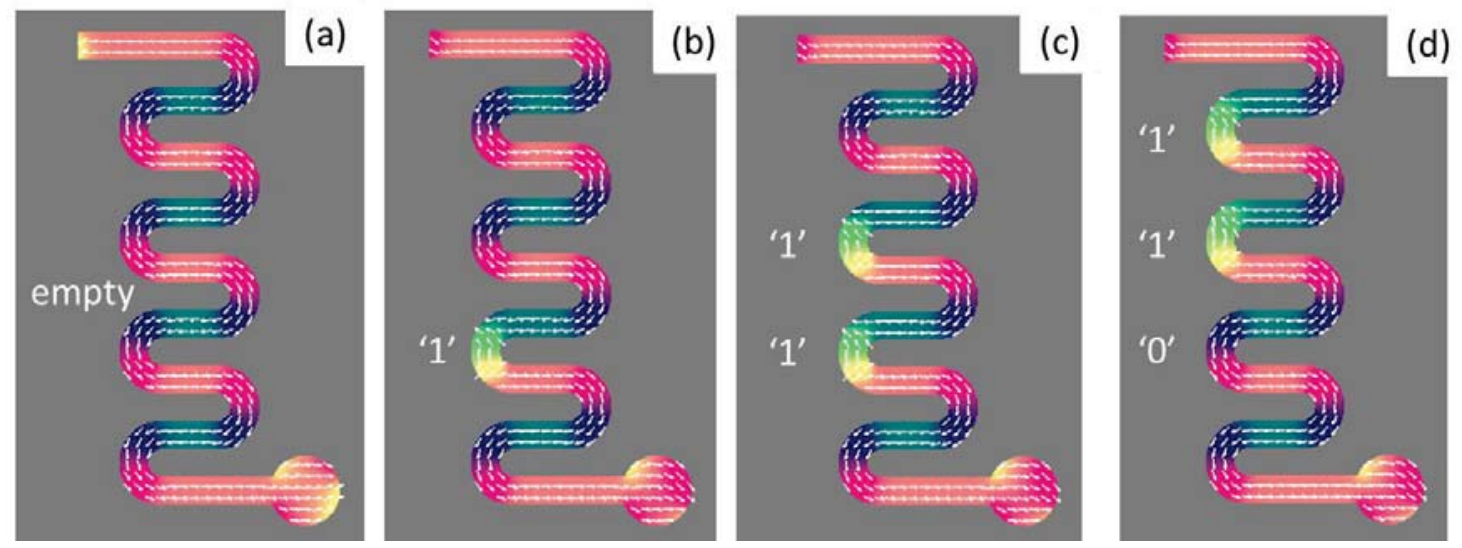

Figure 4.2.7 The magnetization process of writing ' $1-1-0$ ' into the empty shift register according to the magnetic field operation paths in Fig. 4.2.6.

(a)

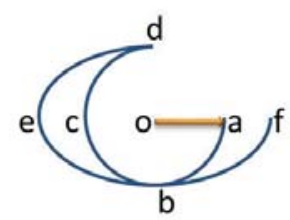

Elimination: $\mathrm{H}_{\mathrm{oa}}=\mathrm{H}_{\mathrm{ob}}=\mathrm{H}_{\mathrm{oc}}=\mathrm{H}_{\mathrm{od}}=0.017 \mathrm{M}_{\mathrm{s}}, \mathrm{H}_{\mathrm{oe}}=\mathrm{H}_{\mathrm{of}}=0.028 \mathrm{M}_{\mathrm{s}}$ a-b-e-d-c-b-f-b-a $\rightarrow$ relax

(b)

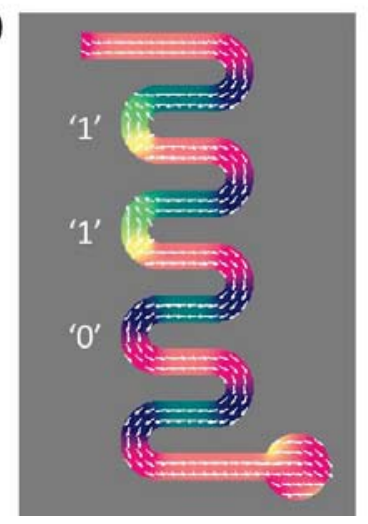

(c)

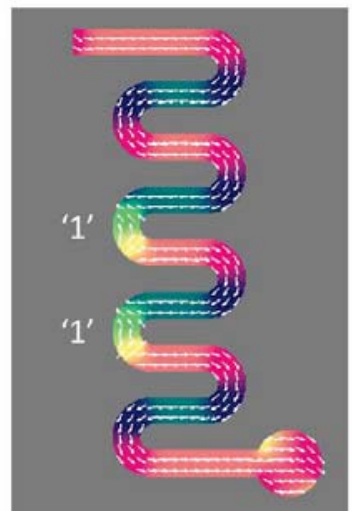

Figure 4.2.8 (a) Applied magnetic field amplitudes and the operation paths to eliminate data from shift register. (b)-(c) The magnetization process of erasing the bit ' 0 ' from the shift register storing ' $1-1-0$ ' under the magnetic field operation paths in (a). 


\subsubsection{Conclusion}

In summary, micromagnetic simulation study demonstrates that a planar magnetic nanowire loop with a shape-isotropic wall generator at one end and a shape-anisotropic wall stopper at the other end functions like a data storage stack: $360^{\circ}$ domain walls are generated and pushed into stack under rotating field before overflow while popped out and annihilated when field rotating direction is inverted until underflow. The stack capacity is determined by the total nanowire loop length. Enlightened by the $360^{\circ}$ domain wall generator, the $360^{\circ}$ domain wall shift register incorporating a shape-isotropic wall generator and a winding nanowire is also proposed with its function confirmed by our simulation results. Unlike the nanowire loop, $360^{\circ}$ domain walls generated by the wall generator are stored within the nanowire corner, which makes the subsequent reading operation easier to be implemented. In addition to providing insights into $360^{\circ}$ domain wall behaviors in planar magnetic nanowires, the simple nanowire structures can be integrated into magnetic circuits as an operation unit for $360^{\circ}$ domain wall generation and storage, with potential applications in miniaturized magnetoelectric devices. 


\subsection{Altering Critical Depinning Current via Domain Wall Pile-up in Magnetic}

Nanowires

\subsubsection{Introduction}

Domain walls in magnetic nanowires attract great attentions for their applications in advanced logic and memory devices [21, 34]. Manipulation of domain walls by electric current is an essential component of domain wall devices and is under intensive study $[19,192]$. Pinning and depinning of domain walls is a critical issue for domain wall manipulation where pinning sites are usually turning corners, bends, joints, and notches in magnetic circuits $[193,194]$. Reducing the critical depinning current is important for technological application, in order to lower the power and to avoid increased nanowire temperature hindering reliable domain wall manipulation [195]. Finding an effective means to reduce and tune the critical depinning current will have a significant impact on the operation of domain wall devices. In this paper we present a micromagnetic simulation study of current-driven domain wall behaviors in thin film-patterned planar magnetic nanowires, which demonstrates a new mechanism for substantial reduction and effective tuning of the critical current for depinning domain walls in magnetic circuit. It is worth noting that micromagnetic simulation has been widely used to understand various factors that affect the critical current for domain wall depinning in magnetic nanowires, including edge roughness [196, 197], thermal perturbation [198], magnetic anisotropy [155] and saturation magnetization [199], applied magnetic field [200], and notch shape [201]. The new mechanism revealed in this work is based on domain wall 
pile-up, in analogy to the well-known dislocation pile-up mechanism responsible for the Hall-Petch effect in mechanical strength.

Since the pinning strength of a pinning site depends on its geometrical shape, the depinning current can be modified by changing the geometrical shape of the pinning site. For example, decreasing the curvature of a bend would reduce its pinning strength [194] and thus lower the critical depinning current. However, changing geometrical shape is constrained by other functional requirements of the pinning site being a part of the magnetic circuit, thus the changeable range of the critical depinning current is limited. Here we show that utilizing domain wall pile-up enables not only a drastic reduction in the critical depinning current but also an effective programing of the current-driven pinning and depinning operations of domain walls. As shown in Fig. 4.3.1(a), a domain wall pile-up is formed in magnetic nanowire when a set of $180^{\circ}$ and/or $360^{\circ}$ domain walls of the same chirality are pushed against a barrier (an s-shape pinning site here). For convenience of discussion, such a domain wall pile-up will be named after its total magnetization rotation angle, $n \pi$-pileup, where $n$ is an integer greater than $2(n=1$ and 2 correspond respectively to $180^{\circ}$ and $360^{\circ}$ domain wall). A $360^{\circ}$ domain wall is highly stable [186] unless an antiparallel magnetic field is applied, under which it dissociates into two $180^{\circ}$ domain walls. Unlike stable $180^{\circ}$ domain wall and relatively stable $360^{\circ}$ domain wall [191], an $n \pi$-pileup is not stable under magnetic field, dissociating into a set of discrete $360^{\circ}$ and $180^{\circ}$ domain walls, as exemplified in Fig. 4.3.1(b). However, an $n \pi-$ pileup under spin-polarized current does not dissociate, but instead moves as a whole in the nanowire, as demonstrated in Fig. 4.3.1(c). When the current-driven motion of an $n \pi-$ 
pileup encounters a barrier in its moving direction, interesting domain wall pinning and depinning phenomena take place, as will be discussed in the following. It is worth noting that a set of $180^{\circ}$ and/or $360^{\circ}$ domain walls of the same chirality (needed for formation of domain wall pile-up) can be conveniently generated in magnetic nanowires [202-204].
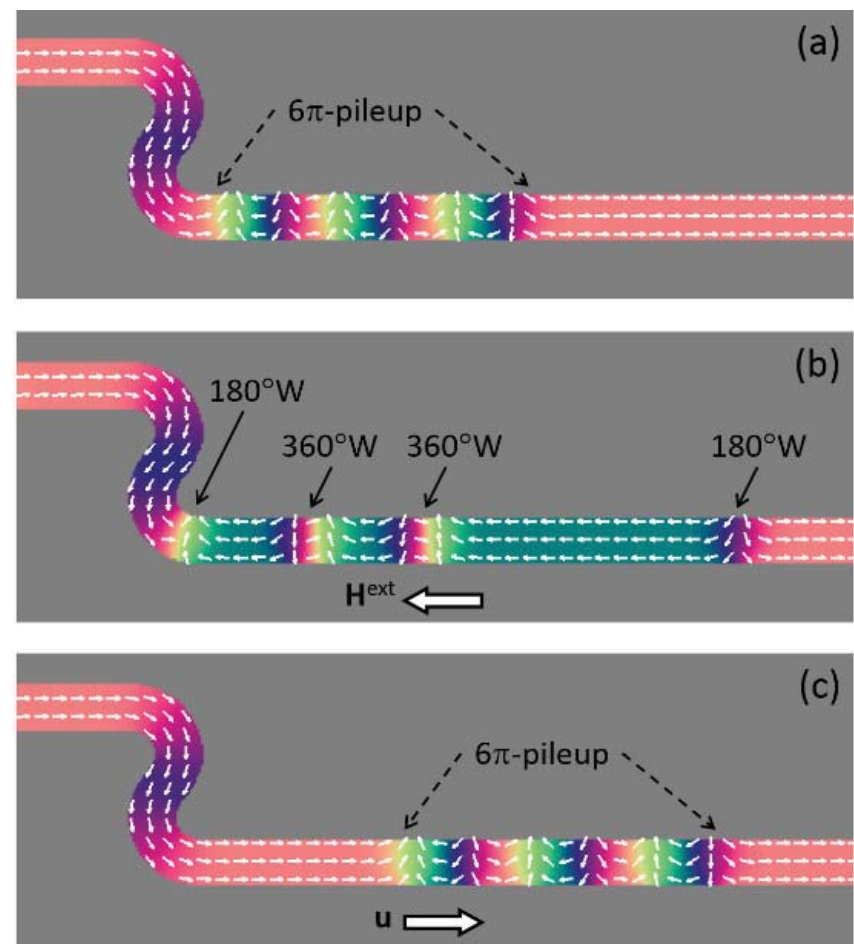

Figure 4.3.1 Micromagnetic simulation of domain wall pile-up. (a) A $6 \pi$-pileup near an s-shape bend. (b) Dissociation of $6 \pi$-pileup into two $180^{\circ}$ and two $360^{\circ}$ domain walls under magnetic field. (c) Current-driven motion of $6 \pi$-pileup under spin-polarized current. Small white arrows and color contours within the nanowire represent magnetization direction.

\subsubsection{Micromagnetic modeling}

Before discussing the important role of domain wall pile-up in current-driven domain wall depinning in nanowires, we first describe the micromagnetic simulation method used in this study. In micromagnetic modeling, magnetic domain structure is 
described by the magnetization direction unit vector field $\mathbf{m}(\mathbf{r})$, which is set to zero outside the magnetic nanowire. The evolution of the magnetic domain structure driven by spin-polarized current and magnetic field is described by the modified Landau-LifshitzGilbert equation [196]:

$$
\dot{\mathbf{m}}=\gamma \mathbf{H}^{\mathrm{eff}} \times \mathbf{m}+\alpha \mathbf{m} \times \dot{\mathbf{m}}-(\mathbf{u} \cdot \nabla) \mathbf{m}+\beta \mathbf{m} \times[(\mathbf{u} \cdot \nabla) \mathbf{m}]
$$

where $\gamma$ is the gyromagnetic ratio, $\alpha$ the damping parameter, $\beta$ the nonadiabatic spintransfer torque coefficient, $\mathbf{H}^{\text {eff }}$ the effective magnetic field, and $\mathbf{u}$ the electron motion velocity vector. The effective magnetic field is determined by the variational derivative of the free energy with respect to the magnetization vector field (i.e., $\mathbf{H}^{\mathrm{eff}}=d F / d \mathbf{m}$ ), which is a sum of exchange energy, magnetostatic energy, and external magnetic energy [20]:

$$
F=A \int|\operatorname{grad} \mathbf{m}(\mathbf{r})|^{2} d^{3} r+\frac{\mu_{0} M_{s}^{2}}{2} f \frac{d^{3} k}{(2 \pi)^{3}}|\mathbf{n} \cdot \mathbf{m}(\mathbf{k})|^{2}-\mu_{0} M_{s} \int \mathbf{H}^{\mathrm{ex}} \cdot \mathbf{m}(\mathbf{r}) d^{3} r
$$

where $A$ is the exchange stiffness constant, $\mu_{0}$ the permeability of vacuum, $M_{\mathrm{s}}$ the saturation magnetization, $\mathbf{H}^{\mathrm{ex}}$ the external magnetic field, $f$ the principal value integral excluding the point $\mathbf{k}=\mathbf{0}, \tilde{\mathbf{m}}(\mathbf{k})=\int \mathbf{m}(\mathbf{r}) e^{-i \mathbf{k} \cdot \mathbf{r}} d^{3} r$, and $\mathbf{n}=\mathbf{k} / k$. The electron motion velocity vector is proportional to the electrical current density $\mathbf{J}$ and determined by $\mathbf{u}(\mathbf{r})=\mathbf{J}(\mathbf{r}) P g \mu_{\mathrm{B}} / 2 e M_{\mathrm{s}}$, where $P$ is the polarization rate of the current and the factor $g \mu_{\mathrm{B}} / 2 e M_{\mathrm{s}}=7 \times 10^{-11} \mathrm{~m}^{3} / \mathrm{C}$ for permalloy [196]. In the simulations, Eq. (4.3.1) is numerically solved for a given magnetic field and electric current condition in a magnetic nanowire of given geometry. A $432 \times 432 \times 1$ computational cell with grid size of $4 \mathrm{~nm}$ is used for the patterned thin film of thickness $D=4 \mathrm{~nm}$, and nanowires with width $W=80 \mathrm{~nm}$ are considered in the simulations. The material parameters of permalloy $\mathrm{Ni}_{80} \mathrm{Fe}_{20}$ are used 
in the simulations: $M_{\mathrm{s}}=860 \mathrm{kA} / \mathrm{m}$ and $A=1.3 \times 10^{-11} \mathrm{~J} / \mathrm{m}[191]$, and $\alpha=0.02$ and $\beta=0.04$ [196].

\subsubsection{Results and Discussion}
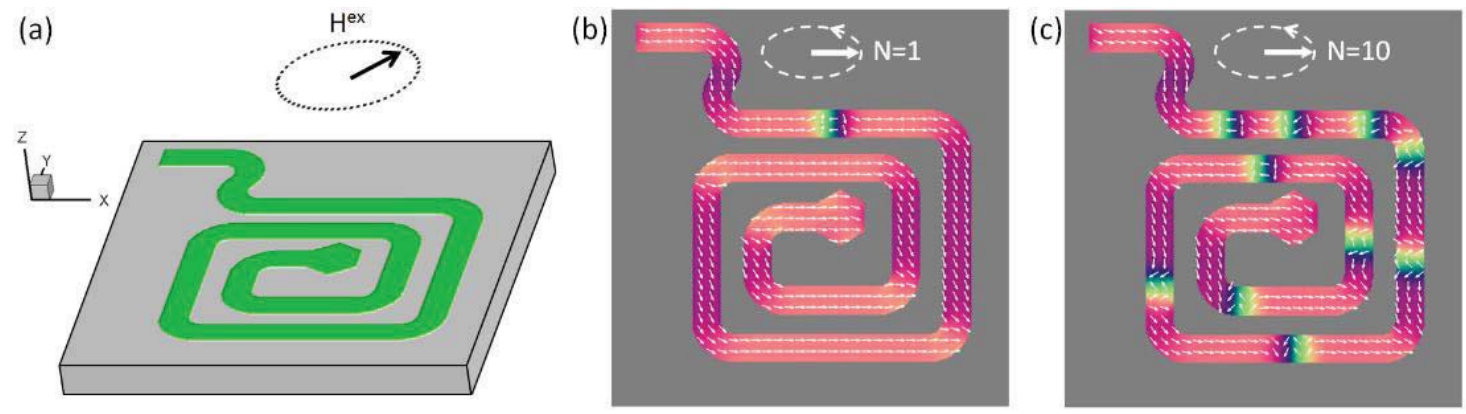

Figure 4.3.2 (a) Planar nanowire of patterned magnetic thin film on nonmagnetic substrate. Simulated generation of $360^{\circ}$ domain walls after (b) 1 cycle and (c) 10 cycles of in-plane counterclockwise rotating magnetic field $\mathbf{H}^{\mathrm{ex}}$.

Computer simulation starts from the generation of multiple domain walls of the same chirality to be used for formation of domain wall pile-up. A magnetic nanowire shown in Fig. 4.3.2(a) is considered, which combines an s-shape bend as pinning site and a nanowire loop as domain wall generator [196]. Under counterclockwise rotating magnetic field, one $180^{\circ}$ domain wall is generated per half cycle in the alternating sequence of head-to-head and tail-to-tail types. All the generated domain walls possess the same chirality (counterclockwise) which is determined by the rotating direction of the applied magnetic field. Under continuously rotating magnetic field, the generated $180^{\circ}$ domain walls propagate along the nanowire loop away from the wall generating element in the center toward the s-shape bend which stops them from propagating further. This 
process leads to the formation of one $360^{\circ}$ domain wall per full cycle of the rotating magnetic field by conjoining a pair of neighboring $180^{\circ}$ domain walls. The number of $360^{\circ}$ domain walls increases with the number of magnetic field cycles as shown in Fig. 4.3.2(b) and (c) respectively for one loading cycle (generating one $360^{\circ}$ domain wall) and ten loading cycles (generating ten $360^{\circ}$ domain walls). Reversing the magnetic field rotating direction reduces the number of domain walls in the nanowire loop by one $360^{\circ}$ domain wall per full cycle of clockwise rotating magnetic field, providing an effective means to control the number of domain walls in the nanowire [196]. The detailed mechanism of domain wall generation, annihilation and storage in such a nanowire design has been studied in our previous work[196]. With a set of domain walls of the same chirality in the nanowire, we consider next the formation of domain wall pile-up at pinning site via current-driven domain wall motion.
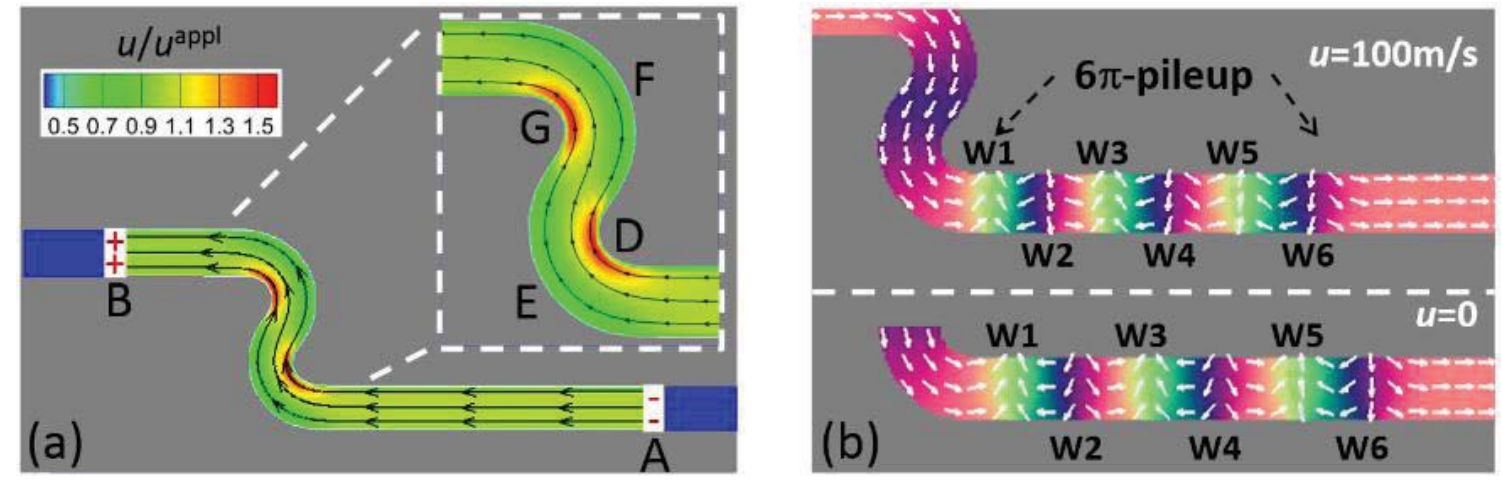

Figure 4.3.3 (a) Current crowding in s-shape bend; magnitude and direction of electron motion velocity vector are visualized by color contours and streamlines. (b) $6 \pi$-pileup formed from three separated $360^{\circ}$ domain walls under $u^{\text {appl }}=100 \mathrm{~m} / \mathrm{s}$ (top) and its relaxation at $u^{\text {appl }}=0 \mathrm{~m} / \mathrm{s}$ (bottom); W1-W6 label the six wall elements each providing $180^{\circ}$ magnetization rotation within the pileup. 
Fig. 4.3.3(a) shows a section of the nanowire near the s-shape bend where the current-driven domain wall pinning and depinning behaviors are to be studied. Electrons enter the nanowire at position A and exit at position B, passing through the s-shape bend. The current density distribution is solved using the phase field method reported previously [205]. The corresponding electron motion velocity vector field is shown by color contours for the magnitude and streamlines for the direction. An inhomogeneous current density distribution develops in a non-straight conducting nanowire. Fig. 4.3.3(a) shows the simulated current crowding at the s-shape bend. Current crowding would influence domain wall depinning behavior as will be analyzed later. Under the applied spin-polarized current that yields electron motion velocity $u^{\text {appl }}=100 \mathrm{~m} / \mathrm{s}$ with the local distribution $\mathbf{u}(\mathbf{r}) / u^{\text {appl }}$ shown in Fig. 4.3.3(a), Eq. (4.3.1) is solved for the magnetic nanowire holding three separated $360^{\circ}$ magnetic domain walls generated by rotating magnetic field as in Fig. 4.3.2(c). Hereafter, the current-driven domain wall motions are studied under zero external magnetic field. The simulation results show that all three $360^{\circ}$ magnetic domain walls are driven by current to move toward the s-shape bend then are stopped there (i.e., pinning), and eventually collide into a $6 \pi$-pileup pushing against the s-shape bend as shown in the upper part of Fig. 4.3.3(b). The individual wall elements each providing $180^{\circ}$ magnetization rotation within the pile-up are labeled $\mathrm{W} 1$ to $\mathrm{W} 6$ in the order of their positions. When the current is removed, the $6 \pi$-pileup remains but relaxes and becomes wider, as shown in Fig. 4.3.1(a) and also in the lower part of Fig. 4.3.3(b) for comparison. As aforementioned, the $6 \pi$-pileup dissociates under magnetic field while moves away from the s-shape bend as a whole upon reversal of the current 
direction, as shown in Figs. 4.3.1(b) and (c), respectively. To push domain walls through the s-shape bend (i.e., depinning), a higher electric current must be applied, leading to complicated pinning and depinning domain wall behaviors as discussed next.
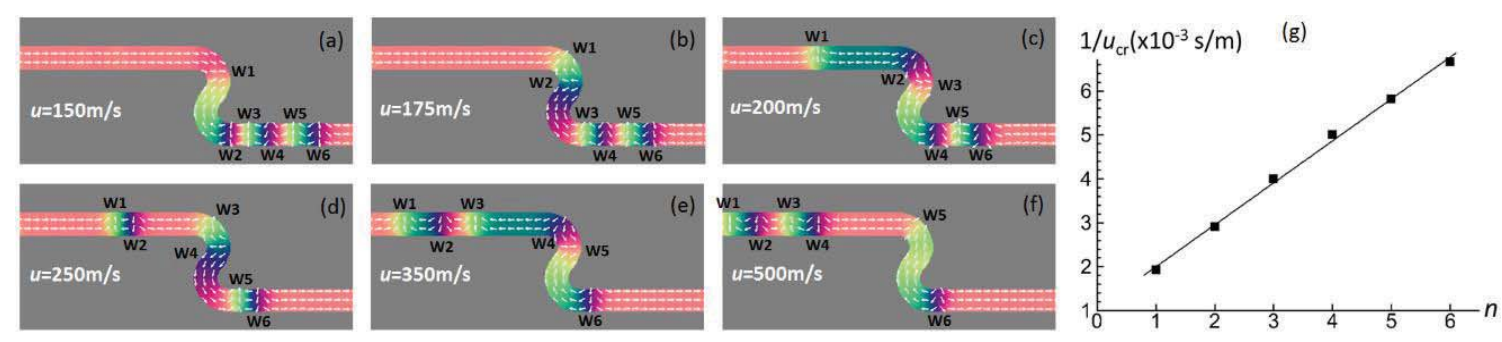

Figure 4.3.4 Simulated domain wall pinning and depinning process at the s-shape bend under increasing current: (a) $u^{\text {appl }}=150 \mathrm{~m} / \mathrm{s}$, (b) $u^{\text {appl }}=175 \mathrm{~m} / \mathrm{s}$, (c) $u^{\text {appl }}=200 \mathrm{~m} / \mathrm{s}$, (d) $u^{\text {appl }}=250 \mathrm{~m} / \mathrm{s}$, (e) $u^{\text {appl }}=350 \mathrm{~m} / \mathrm{s}$, and (f) $u^{\text {appl }}=500 \mathrm{~m} / \mathrm{s}$. (g) Dependence of the critical depinning current $u_{\mathrm{cr}}$ on the number of domain walls $n$ in the pile-up.

Fig. 4.3.4 shows the simulated domain wall pinning and depinning behaviors as the current increases. The s-shape bend is composed of two oppositely curved segments: the lower segment turns clockwise while the upper segment counterclockwise. As will be explained, the lower segment acts as a potential barrier to block domain walls from entering the s-shape bend while the upper segment acts as a potential well that retains domain walls from exiting the bend. At $u^{\mathrm{appl}}=150 \mathrm{~m} / \mathrm{s}$ shown in Fig. 4.3.4(a), the first wall (W1) enters the bend leaving behind the rest of walls (W2-W6) in the pile-up; it passes through the lower segment to reach the upper segment and is pinned there. At $u^{\text {appl }}=175 \mathrm{~m} / \mathrm{s}$ in Fig. 4.3.4(b), the second wall (W2) follows the same path into the sshape bend and joins W1. At $u^{\text {appl }}=200 \mathrm{~m} / \mathrm{s}$ in Fig. 4.3.4(c), the third wall (W3) follows again the same path into the s-shape bend and joins W2 while W1 is pushed out of the 
bend into the upper straight segment beyond the s-shape bend. In the same manner, when the current is further increased to $u^{\text {appl }}=250 \mathrm{~m} / \mathrm{s}$ and $350 \mathrm{~m} / \mathrm{s}$ in Fig. $4.3 .4(\mathrm{~d})$ and (e), the fourth wall (W4) and fifth wall (W5) respectively enter the s-shape bend while W2 and W3 are pushed out of the bend into the upper straight segment. Now three walls (W1, W2, W3) have been transported into the upper straight segment after passing through the s-shape bend, and the rest two walls (W4, W5) are pinned inside the s-shape bend while the last wall (W6) remains blocked from entering the s-shape bend. When the current is further increased to $500 \mathrm{~m} / \mathrm{s}$ in Fig. 4.3.4(f), W4 is pushed out of the s-shape bend while W5 is still pinned inside and W6 is still blocked from entering the s-shape bend. This process demonstrates that the critical current for depinning domain walls of an $n \pi$-pileup strongly depends on the number of domain walls, $n$, in the pile-up. For example, depinning wall W1 to enter the s-shape bend requires $u^{\text {appl }}=150 \mathrm{~m} / \mathrm{s}$ for $n=6$ in Fig. 4.3.4 (a) while $u^{\text {appl }}=500 \mathrm{~m} / \mathrm{s}$ is not high enough for depinning wall W6 for $n=1$ in Fig. 4.3.4(f). To depin a wall to exit the s-shape bend, it requires two walls inside the s-shape bend. For example, $u^{\text {appl }}=200 \mathrm{~m} / \mathrm{s}$ can push $\mathrm{W} 1$ out when there is another wall behind inside the s-shape bend as shown in Fig. 4.3.4(c) while $u^{\text {appl }}=500 \mathrm{~m} / \mathrm{s}$ is not high enough to push wall W5 out when it is the only wall pinned inside the s-shape bend as shown in Fig. 4.3.4(f).

To quantitatively determine the dependence of the critical current $\left(u_{\mathrm{cr}}\right)$ on the number of walls $(n)$ in the pile-up, systematic simulations are performed by considering six cases of different domain wall pile-ups with $n=1,2, \ldots, 6$, respectively. Fig. 4.3.4(g) presents the simulation results of $u_{\mathrm{cr}}$ that is required to depin the first wall of the pile-up to enter the s-shape bend. It is shown that the dependence of the critical current on the 
number of walls in the pile-up approximately follows an inverse linear relationship of $u_{\text {cr }}$ $\sim 1 / n\left(\right.$ or $\left.1 / u_{\text {cr }} \sim n\right)$. This result can also be understood by analyzing the pinning force to the pile-up and the interaction forces among the individual domain walls in the pile-up, using the force balance equation $(\beta / \lambda) u-f_{\text {pin }}=0$ from the simple one-dimensional domain wall motion model [197] where the driving force from the current is balanced by the pinning force $f_{\text {pin }}$ for one static domain wall with width parameter of $\lambda$. For the case of $n=1$ in Fig. 4.3.4(f), W6 experiences the current-induced driving force $(\beta / \lambda) u$ to the left, which is balanced by the pinning force caused by the barrier at the entrance of the sshape bend. When the current is increased to overcome the barrier of strength $f_{0}$, W6 would be depinned and move into the s-shape bend, and the critical current is $u_{\mathrm{cr}}(n=1)=(\lambda / \beta) f_{0}$ as determined by the force balance equation. For the case of $n=2$ in Fig. 4.3.4(d), W6 experiences the current-induced driving force $(\beta / \lambda) u$ to the left and the balancing wall-wall interaction force $f_{\mathrm{w} 6}=(\beta / \lambda) u$ to the right exerted by W5; on the other hand, W5 experiences the current-induced driving force $(\beta / \lambda) u$ to the left and the wall-wall interaction force $f_{\mathrm{w} 6}=(\beta / \lambda) u$ to the left exerted by $\mathrm{W} 6$, and their sum is balanced by the pinning force $f_{\text {pin }}$ from the barrier. Therefore, the critical current required to overcome the barrier of strength $f_{0}$ is now $u_{\mathrm{cr}}(n=2)=(\lambda / 2 \beta) f_{0}$, one half of $u_{\mathrm{cr}}(n=1)$. Following the same procedure, it is shown that the critical current for an $n \pi$ pileup is $u_{\mathrm{cr}}(n)=(\lambda / n \beta) f_{0} \sim 1 / n$, in agreement with the simulation results shown in Fig. 4.3.4(g). In other words, each individual domain wall in the $n \pi$-pileup contributes to the 
front domain wall a current-induced driving force of $(\beta / \lambda) u$ through wall-wall interaction force, which effectively reduces the critical current by a factor of $n$. Such a strong dependence of $u_{\mathrm{cr}} \sim 1 / n$ offers an effective means to drastically reduce and tune the depinning current by simply controlling the number of walls in a pile-up.

As the pinning strength of a curved nanowire segment depends on its curvature [194], the curvatures of the lower and upper segments of the s-shape bend can be respectively modified to further tailor its pinning effect. Fig. 4.3.5(a) shows the domain wall structures of head-to-head and tail-to-tail $180^{\circ}$ domain walls of counterclockwise chirality in straight nanowire segment, as generated by the rotating magnetic field in Fig. 4.3.2. For both types, domain wall width is not uniform across the planar nanowire: wider on the upper edge and narrower on the lower edge, exhibiting an upright bowl shape [206]. Due to such asymmetric domain wall structure, curving the nanowire upward and downward would result in different changes in domain wall structure and energy. For example, upward curvature shortens the upper edge and lengthens the lower edge opposing the natural bowl-shaped domain wall, while downward curvature lengthens the upper edge and shortens the lower edge accommodating the natural bowl-shaped domain wall. The former case corresponds to an increased magnetization rotation angle $(\pi+\Delta)$ and increased domain wall energy $(\Delta E>0)$, as shown in Fig. 4.3.5(b) compared to Fig. 4.3.5(a); the latter case, on the other hand, corresponds to a decreased magnetization rotation angle $(\pi-\Delta)$ and decreased domain wall energy $(\Delta E<0)$, as shown in Fig. 4.3.5(c) compared to Fig. 4.3.5(a). While Fig. 4.3.5(b) and (c) show the effects of nanowire 
curvature on domain wall structure and energy of head-to-head type of counterclockwise chirality, the same effects are also observed in tail-to-tail type of the same chirality.
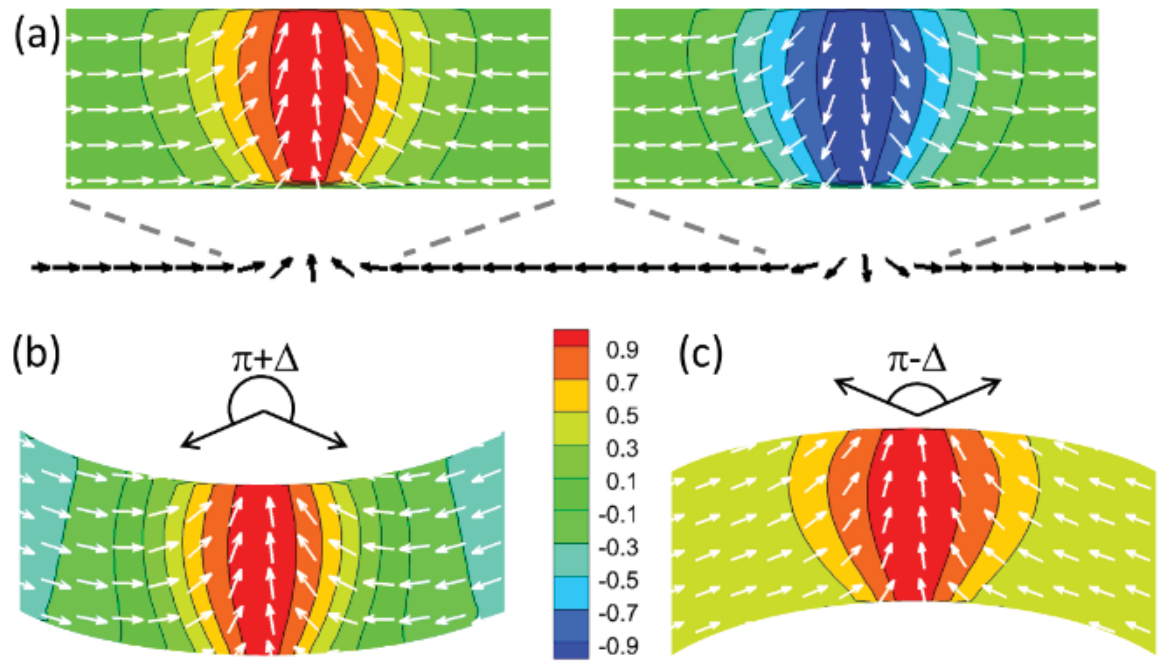

Figure 4.3.5 Domain wall structures in a straight nanowire segment (a), and two oppositely curved nanowire segments with (b) increased domain wall width and energy and (c) reduced domain wall width and energy. Small white arrows represent magnetization vectors and color contours represent their components parallel to the domain wall.

Due to an increased domain wall energy $(\Delta E>0)$ shown in Fig. 4.3.5(b), the lower segment of the s-shape bend in Fig. 4.3 .4 acts as a potential barrier [194], blocking domain walls in the lower straight nanowire from entering the bend; and due to a decreased domain wall energy $(\Delta E<0)$ shown in Fig. 4.3.5(c), the upper segment of the sshape bend in Fig. 4.3.4 acts as a potential well [194], pinning domain walls inside the bend from exiting into the upper straight nanowire. Therefore, with the lower segment being a potential barrier and the upper segment being a potential well, the s-shape bend serves as pinning element to domain wall motions in the nanowire, as observed in Fig. 
4.3.4. Domain wall energy depends on both the curvature and the width of the nanowire. When the nanowire width is much smaller than the radius of curvature, the dependence of domain wall energy on the curvature can be described by a simple relationship $|\Delta E| \sim 2 \pi A / R$, where $A$ is the exchange stiffness constant and $R$ the radius of curvature. Correspondingly, the critical depinning current required to overcome such an energy barrier would show a similar proportional dependence on the curvature, $J_{\mathrm{cr}} \sim 1 / R$. When the radius of curvature is comparable to the width of the nanowire, the domain wall energy change and the critical depinning current would deviate from this simple linear dependence on the curvature $1 / R$, which can be evaluated numerically by computer simulations; nevertheless, the critical depinning current would increase when the curvature increases. Figure 4.3.6 shows the influence of the bend curvature on domain wall pinning strength by considering two s-shape bends of different curvatures, $1 / R=1 / 80 \mathrm{~nm}^{-1}$ and $1 / 160 \mathrm{~nm}^{-1}$ ( $R$ being the radius of the nanowire centerline); the former has a higher curvature and is the same as in Fig. 4.3.4, while the latter has a smaller curvature thus is bigger in size. The simulation starts with a pinned $360^{\circ}$ domain wall in the lower straight nanowire which consists of two wall elements, W5 and W6, as shown in Fig. 4.3.4(d), and $u^{\mathrm{appl}}=400 \mathrm{~m} / \mathrm{s}$ is applied. For the smaller s-shape bend in Fig. 4.3.6(a), as expected from the results in Figs. 4.3. 4(e) and (f), the first wall passes through the lower segment to enter the s-shape bend and is subsequently pinned inside by the upper segment, while the second wall remains blocked in the lower straight nanowire. For the larger s-shape bend in Fig. 4.3.6(b), both walls pass through the lower and upper segments of the s-shape bend and move into the upper straight nanowire. It shows that the 
critical depinning current for the larger s-shape bend is lower than $400 \mathrm{~m} / \mathrm{s}$, compared with that higher than $500 \mathrm{~m} / \mathrm{s}$ for the smaller s-shape bend.

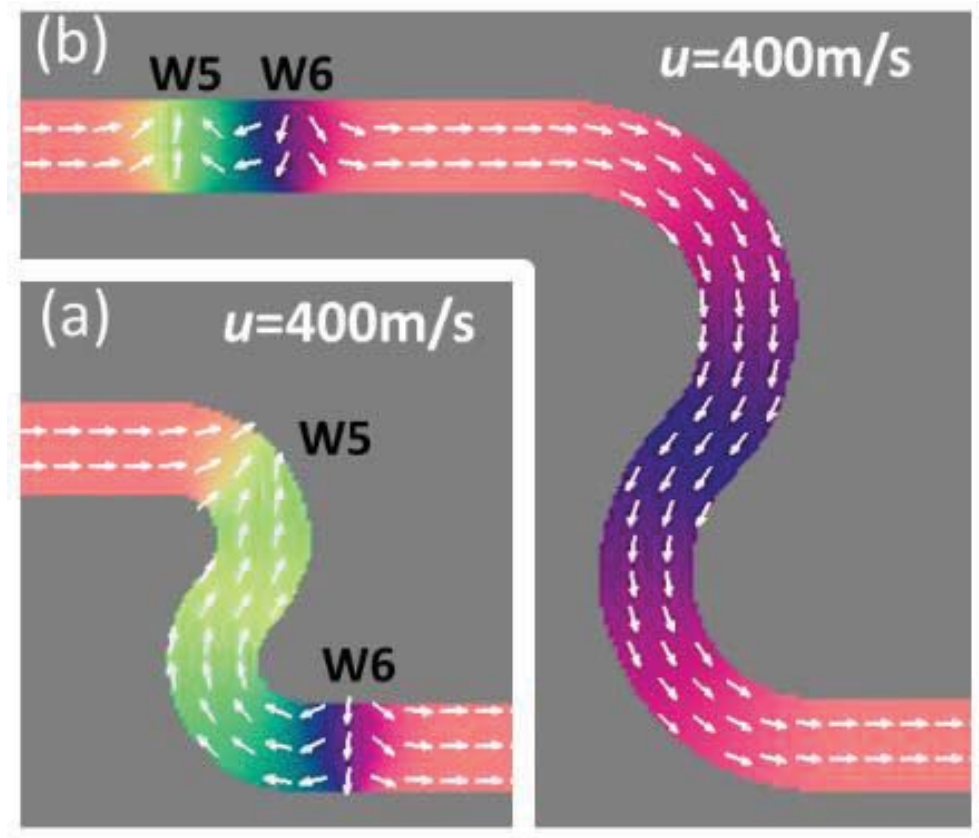

Figure 4.3.6 Domain wall behaviors under $u^{\text {appl }}=400 \mathrm{~m} / \mathrm{s}$ at two s-shape bends of different curvatures: (a) $1 / R=1 / 80 \mathrm{~nm}^{-1}$ and (b) $1 / R=1 / 160 \mathrm{~nm}^{-1}$.

Finally, pinning sites (turning corners, bends, joints, and notches) involve certain geometrical shape changes that usually cause current crowding (heterogeneous current density distribution). For example, the current density is high near D and G while low near $\mathrm{E}$ and $\mathrm{F}$ positions in the s-shape bend in Fig. 4.3.3(a). Based on Eq. (4.3.1), the nonuniformity in current density distribution would influence the current-driven domain wall behaviors. To distinguish the effects of the current density distribution from that of the geometrical shape of the pinning site (e.g., the curvature) on domain wall behaviors, a head-to-head domain wall in a straight nanowire under different current density 
distribution conditions is simulated. Under given average value of $u^{\text {app }}=300 \mathrm{~m} / \mathrm{s}$, three artificial current density distribution conditions are considered as shown in Fig. 4.3.7: case I with uniform current density, case II with higher current density on the lower edge (the narrow side of the domain wall) and case III with higher current density on the upper edge (the wide side of domain wall) that linearly decreases to zero across the nanowire width. The simulation results show that, in all three cases, the domain wall moves to the right as expected, but with different speeds. The domain wall moves at higher speed in case II $(645 \mathrm{~m} / \mathrm{s})$ while at lower speed in case III $(537 \mathrm{~m} / \mathrm{s})$ than in case I $(600 \mathrm{~m} / \mathrm{s})$. Moreover, changes in domain wall structure due to different current density distributions are also observed. Three snapshots of the moving domain walls in the three cases are shown in Fig. 4.3.7. The upright bowl-shaped domain wall structure in case I shown in Fig. 4.3.7(a) is skewed to the left in case II in Fig. 4.3.7(b) while to the right in case III in Fig. 4.3.7(c), complying with the simple idea that the part of a wall under higher current density is pushed more than the part under lower current density. The observed differences in domain wall speed in the three cases (III, I, II in an increasing order) indicate that the current provides a greater driving force to the wall motion as a whole when the current density is higher at the narrow side than at the wide side of the domain wall. It is because the narrow side possesses a greater magnetization gradient $(\nabla \mathbf{m})$ thus experiences a higher driving force to the current-driven domain wall motion, since the driving force is proportional to the product of the current density and the magnetization gradient as shown in Eq. (4.3.1). Therefore, locally distributing a higher current density at the narrow part (with greater magnetization gradient) of the domain wall would increase the driving force to the domain wall motion and thus reduce the critical depinning current 
as an average current density. Moreover, as different types of domain walls possess different wall structures, the same current density would affect different types of domain walls differently. For example, if the chirality of the domain wall in Fig. 4.3.7 is switched to the opposite (clockwise), the domain wall structure changes to upside-down bowl shape and the domain wall speed would be higher in case III than in case II instead. Since the current crowding is always present at a pinning site and the current density distribution is determined by the shape of the pinning site, the effects of the current crowding can be utilized by appropriate design of the pinning site geometry and choice of domain wall chirality.

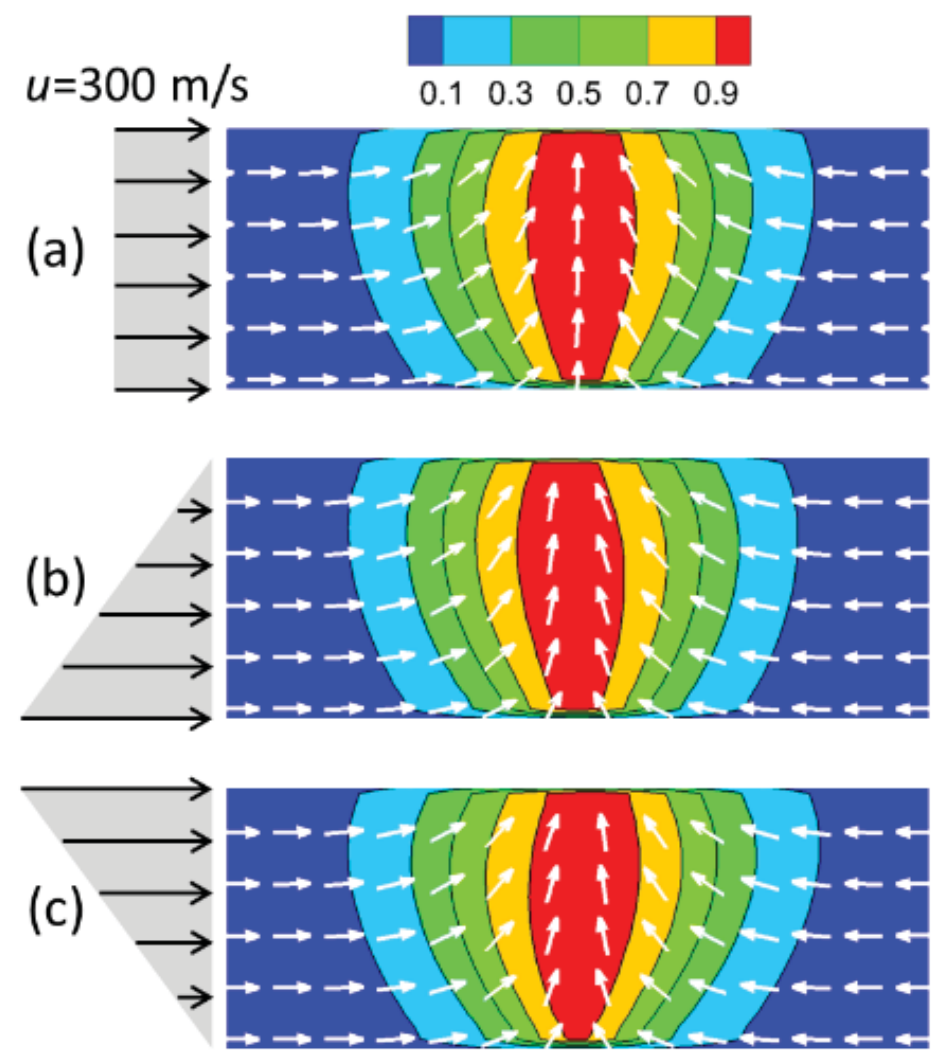

Figure 4.3.7 Simulated domain wall structure and motion under (a) uniform, (b) linearly increasing, and (c) linearly decreasing current density across the width of the nanowire. 


\subsubsection{Conclusion}

In summary, this paper reports an important role of magnetic domain wall pile-up in current-driven domain wall depinning behaviors in magnetic nanowires, which is analogous to the crucial role of dislocation pile-up in determining the mechanical strength of polycrystalline materials (Hall-Petch effect). The critical current for domain wall depinning can be drastically reduced and effectively tuned by simply controlling the number of domain walls in the pile-up. It is shown that domain wall pile-up can be formed by conjoining multiple domain walls of the same chirality, which can be conveniently generated, as exemplified in a magnetic nanowire loop under rotating magnetic field. An s-shape bend functions as a pinning site in the simulations, which consists of two oppositely turning curved segments that respectively serve as potential barrier and potential well. The dependence of the pinning strength on the curvature of the bend and the effects of the heterogeneous current density distribution at the pinning site on the domain wall behaviors are discussed. 


\subsection{Current Induced Domain Wall Depinning and Transportation through Planar Nanowire Circular Geometry by Spin Transfer Torques and Spin-orbit Torques}

\subsubsection{Introduction}

Pinning effects which are commonly existed in magnetic materials may significantly influence the domain wall (DW) motion behaviors driven by magnetic field or spin-polarized current. Among those pinning effects, artificially introduced geometrical pinning sites, such as notches [21, 22, 180, 193, 207], protrusions [193, 208, 209], cross- or T-shaped traps [35, 210, 211], zigzag corners [36, 212-214] and loop corners [32-34, 215, 216], play a very important role in magnetic planar nanowires and DW-based devices. For the zigzag nanowire or nanowire circuits in particular, circular geometries like round corners always exist, especially for the most widely studied Permalloy planar nanowires. [32-34, 47, 175, 194, 211, 215-218] Unlike other pinning sites, a corner with circular geometry often keeps the same width with the straight nanowire part, and it usually performs as either a pinning barrier or a pinning well which depends on the chirality of a transverse DW, and DW depinning and transportation through those round corners are crucial for DW-based memory and logic elements [21, 32, 34]. Thus, current induced depinning and transportation of transverse DWs through round corners will be focused in this paper.

The conventional spin-transfer torques (STTs) include both adiabatic and nonadiabatic torques $[3,5,8]$, which are due to the coupling between magnetizations and spin-polarized current and able to drive the DW moving in the direction of conduction electrons, i.e., the opposite direction of current. An alternative way to manipulate 
magnetization dynamics is through the current induced spin-orbit torques (SOTs) which are usually contributed by two main effects, Rashba effect (RE) [62] and Spin-Hall effect (SHE) [63]. Both effects are derived from a nonmagnetic heavy metal layer with strong SO interactions. For RE, a typical system is composed by SO/ferromagnetic (FM)/oxide, and the RE is caused by the interfacial electric field which is usually perpendicular to the surface due to the symmetry breaking at the interface. $[37,38,65,66]$ Since RE can give rise to an effective transverse field perpendicular to both electric field and injected current directions, the RE related torque is also called field-like torque [67, 68]. For SHE, the spin up and spin down electrons in the SO layer will deflect in opposite directions toward the interface to form a transverse spin current which will be injected into the FM layer to exert a torque. The SHE related torque is also called Slonczewski torque, which can act as an anti-damping torque to make the DW move along the current direction. [67, 68, 70-75] Therefore, the interface-derived SOTs can significantly influence the DW motion behaviors, and then DW depinning and transporation from round corners by SOTs besides of STTs will also be taken into account in this paper.

In this paper, we will theoretically analyze the critical current for the DW to depin or transport through round corners by STTs and SOTs based on the one-dimensional (1D) model combining with the numerical calculations. Although some theoretical calculations for current induced DW depinning from extrinsic pinning sites of general form have been done [61, 74, 76, 197], detailed calculations for specific pinning sites like round corners are still lacking. Our calculations can provide an approach to analyze the DW depinning and transportation behaviors through pinning sites with circular geometry. 


\subsubsection{Theory}

The magnetization dynamics driven by spin-polarized current can be described by the modified Landau-Lifshitz-Gilbert equation including both STTs and SOTs $[51,69,72,76]:$

$$
\frac{\partial \mathbf{m}}{\partial t}=-\gamma \mathbf{m} \times\left(\mathbf{H}_{\mathrm{eff}}+\mathbf{H}_{R}\right)+\alpha \mathbf{m} \times \frac{\partial \mathbf{m}}{\partial t}-(\mathbf{u} \cdot \nabla) \mathbf{m}+\beta \mathbf{m} \times[(\mathbf{u} \cdot \nabla) \mathbf{m}]-\alpha_{S H} \mathbf{m} \times[\mathbf{m} \times \hat{\sigma}],
$$

where $\gamma$ is the gyromagnetic ratio, $\alpha$ the damping parameter, $\beta$ the nonadiabatic spintransfer torque coefficient, $\mathbf{H}_{\text {eff }}$ the effective magnetic field, $\mathbf{H}_{R}$ the Rashba effective field, $\mathbf{u}$ the electron motion velocity vector, $\alpha_{S H}$ the parameter determining the amplitude of SHE, and $\hat{\sigma}$ the unit vector denoting the SHE spin direction as shown in Fig. 4.4.1. The effective magnetic field is determined by the variational derivative of the free energy with respect to the magnetization vector field, $\mathbf{H}_{\mathrm{eff}}=\delta F / \delta \mathbf{m}$. The electron motion velocity vector proportional to the electrical current density $\mathbf{J}$ is determined by $\mathbf{u}(x)=-\mathbf{J}(x) P g \mu_{B} /\left(2 e M_{s}\right)$ where $P$ is the polarization rate of the current and the factor $g \mu_{\mathrm{B}} / 2 e M_{\mathrm{s}}=7 \times 10^{-11} \mathrm{~m}^{3} / \mathrm{C}$ for Permalloy [51]. The Rashba effective field is given by

$$
\mathbf{H}_{R}(x)=\frac{\alpha_{R} P}{\mu_{0} \mu_{B} M_{s}} \hat{z} \times \mathbf{J}(x)=\frac{2 e \alpha_{R}}{g \mu_{B}^{2}} \mathbf{u}(x) \times \hat{z}=-\frac{2 e \alpha_{R} u}{g \mu_{B}^{2}} \hat{\sigma},
$$

where $\alpha_{R}$ is the Rashba parameter. $\alpha_{S H}$ is given by

$$
\alpha_{S H}=\frac{\gamma{ }^{2} \theta_{S H} J}{2 e \mu_{0} M_{s} L_{z}}
$$


where $L_{z}$ is the FM layer thickness and $\theta_{S H}$ is the Spin-Hall angle denoted as the ratio of the spin current to the charge current densities and its value can be either positive or negative depending on the SO layer material $[70,71]$.

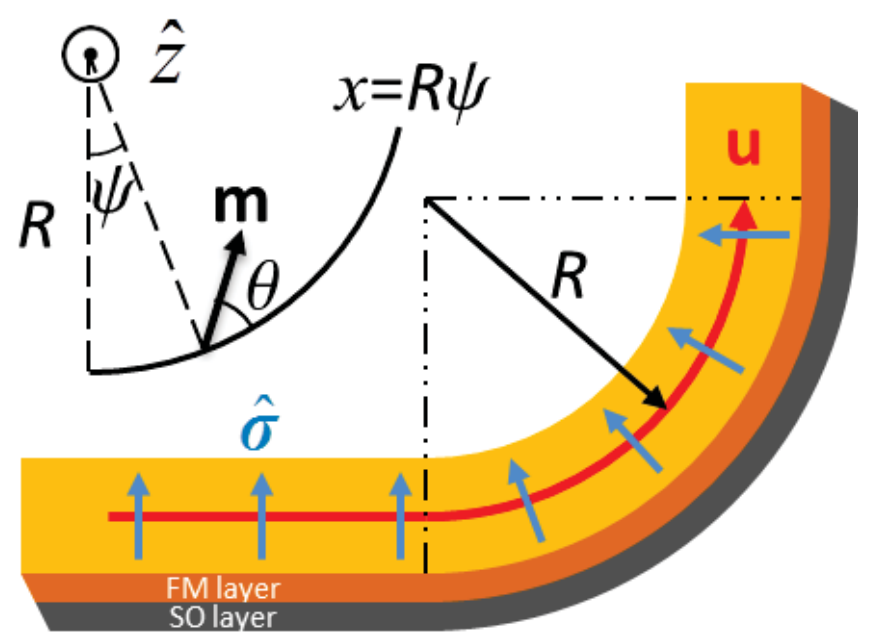

Figure 4.4.1 Schematics of a planar FM nanowire (yellow color) with a round corner of circular geometry deposited on the SO layer (gray color). The average radius of the round corner is denoted as $R$. The red line represents the trace of the effective injected current density $\mathbf{u}$. Blue arrows represent the SHE spin directions denoted as $\hat{\sigma}$. The inset at the upper left shows a cylindrical coordinate used to describe the magnetization system within round corner, where $\hat{z}$ axis is perpendicular to the nanowire surface.

Fig. 4.4.1 shows schematically the planar FM nanowire with a round corner of circular geometry deposited on the SO layer. $R$ denotes the radius of the round corner. Although the distribution of the current density is not homogeneous within the corner, an effective current density along the nanowire geometry can be assumed for relatively large $R$ (much larger than DW width and nanowire width), which is shown by the red line in Fig. 4.4.1. For the calculations in the following sections, magnetic parameters of 
Permalloy material are adopted [179]: $M_{s}=860 \mathrm{kA} / \mathrm{m}$, magnetocrystalline anisotropy $K_{1}=0$, exchange constant $A=1.3 \times 10^{-11} \mathrm{~J} / \mathrm{m}$, damping parameter $\alpha=0.02$. The planar nanowire with thickness of $4 \mathrm{~nm}$ and width of $80 \mathrm{~nm}$ is considered, and then the shape anisotropy energies can be approximated as $K_{z} \approx 0.9 K_{d}$ and $K_{\sigma} \approx 0.1 K_{d}$, where $K_{d}=0.5 \mu_{0} M_{s}^{2} .[1]$

\subsubsection{Results and Discussion}

\subsubsection{Spin Transfer Torques}

In order to study the current induced DW depinning or transportation through round corners, the pinning effect of the round corner should be studied first. A round corner with $\Delta \psi=\pi / 2$ and radius $R$ is taken into account. The total free energy density including exchange and shape anisotropy energies for magnetizations within the nanowire is

$$
e_{t o t}=A\left(\theta^{\prime}+1 / R\right)^{2}+K \sin ^{2} \theta
$$

where $K=K_{\sigma}$ and $\theta$ is the magnetization angle with respect to the nanowire geometry trace as shown in Fig. 4.4.1. It is noted that Eq. (4.4.4) will fall into the case of straight nanowire at $R \rightarrow \infty$. The DW structure can be obtained by solving $\delta e_{t o t} / \delta \theta=0$, and then we have

$$
\theta^{\prime}=\sin \theta / \lambda,
$$

where $\lambda=\sqrt{A / K}$ is the DW width parameter which is independent of $R$ and DW chirality. The total energy is given by 


$$
E=\int e_{t o t} d x=A /\left.R\right|_{\psi_{1}} ^{\psi_{2}}+\left.(-2 \sqrt{A K} \cos \theta+2 A / R)\right|_{\theta_{1}} ^{\theta_{2}}
$$

Fig. 4.4.2 (a)-(f) show the total energies of magnetization structures within either straight or circular nanowire geometries. Obviously, the curve effect alone will increase the total energy and the energy increase is proportional to the angle change $\Delta \psi$, as shown in Fig. 4.4.2 (a) and (d). It is worth noted that the DW energies within the straight wire and the round corner are quite different, and their dependences on DW chirality are also different, i.e., the DW energy for the straight geometry is independent of DW chirality (Fig. 4.4.2 (b) and (c)) while for the corner geometry it depends on DW chirality (Fig. 4.4.2 (e) and (f)). When both the magnetization and round corner rotate in the same sense, either clockwise $(\mathrm{cw})$ or counterclockwise $(\mathrm{ccw})$, the DW energy is higher than in the straight wire and the round corner acts as a pinning barrier, which can be denoted as Type I DW (Fig. 4.4.2 (e)). On the contrary, when they rotate in the opposite senses, the DW energy is lower and the round corner acts as a pinning well, which can be denoted as Type II DW (Fig. 4.4 .2 (f)).

As for such a pinning barrier or pinning well, the energy change for the DW from straight wire to round corner can be described by

$$
\Delta E(x)=\frac{2 A}{R} \theta(x)
$$

as shown in Fig. 4.4 .3 (a) and (c) for Type I and II, respectively. For either Type I or Type II, in order to transport through the round corner, the DW has to overcome a potential rising and also experience a potential falling (A and B), and the velocity will be either decelerated or accelerated. Fig. 4.4 .3 (b) and (d) show the DW transportation process under $u=225 \mathrm{~m} / \mathrm{s}$ with $\beta=2 \alpha=0.04$ and $R=8 \lambda$ for Type I and Type II DW, 
(a)

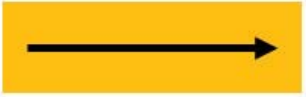

$E=0$

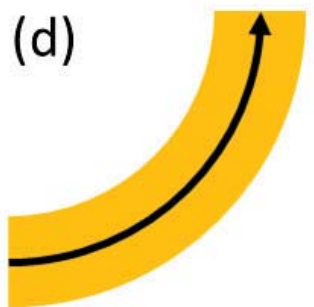

(b)

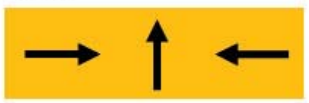

$E=4 \sqrt{A K}$

(e)
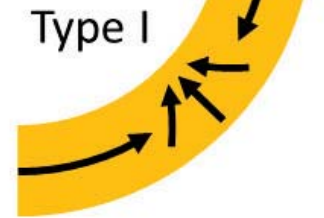

(c)

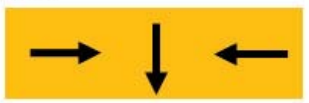

$E=4 \sqrt{A K}$

$$
E=\frac{\pi A}{2 R} \quad E=\frac{\pi A}{2 R}+4 \sqrt{A K}+\frac{2 \pi A}{R} \quad E=\frac{\pi A}{2 R}+4 \sqrt{A K}-\frac{2 \pi A}{R}
$$

Figure 4.4.2 Schematics of magnetic domain and DW structures in nanowire with (a)-(c) straight and (d)-(f) circular geometries. The corresponding total energies calculated according to Eq. (4.4.6) are shown in each figure's bottom.

respectively. As is expected, the time window for the DW to overcome the rising edge is larger than the falling edge, and the Type II DW can transport through the corner more easily than Type I due to the relatively short corner length. One can expect that, when the corner radius $R$ is large enough, the DW will have enough time to restore the previous steady motion state after passing corner edge A, and then both Type I and II DWs will have the same critical current $u_{c}$ to transport through the whole corner, whose value is determined by the rising edge, i.e., $u_{c}$ is determined by edge A for Type I DW while by edge B for Type II DW. For the following calculations, therefore, only the rising edge or the energy barrier is considered in order to calculate the threshold current for the depinning and transportation of DW. 
(a)

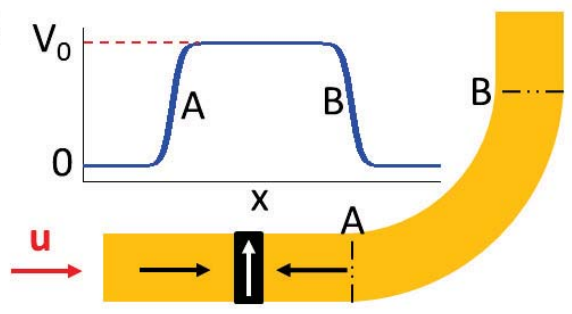

(b)

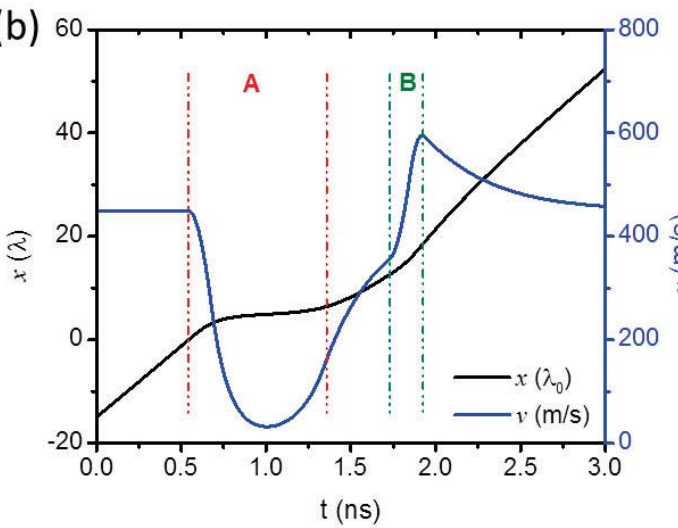

(c)

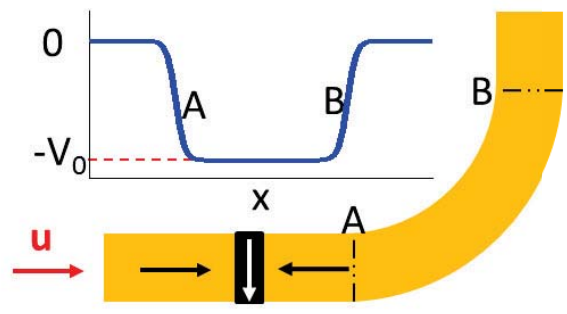

(d)

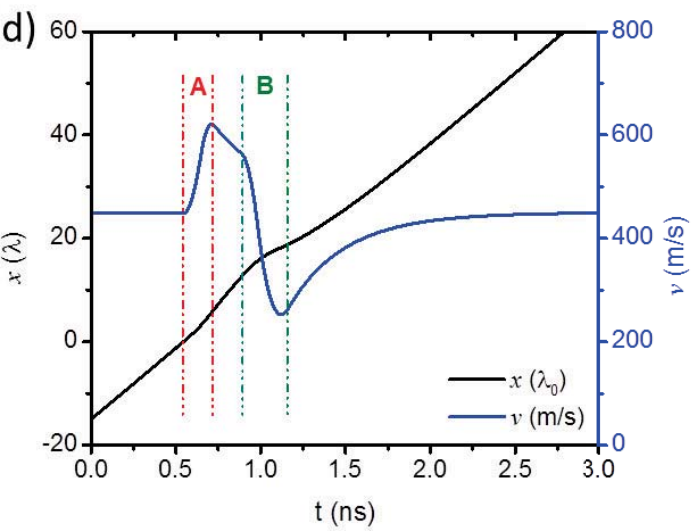

Figure 4.4.3 The pinning potential profiles for (a) Type I and (b) Type II DW across the round corner, where $\mathrm{A}$ and $\mathrm{B}$ represent the potential rising or falling edge. The DW transportation process under $u=225 \mathrm{~m} / \mathrm{s}$ with $\beta=2 \alpha=0.04$ and $R=8 \lambda$ for (b) Type I and (d) Type II DW, where the black line and the blue line represent the DW position and velocity, respectively.

Since the energy barrier in the form of Eq. (4.4.7) is difficult to make an analytical derivation for the DW motion behavior, an approximation extended to the second order is made, i.e., the pinning force in the form extended to the first order is considered. In that approximation, the maximum energy barrier $V_{0}$ is kept, and then the energy barrier will be

$$
\begin{aligned}
& V(x)=\frac{V_{0}}{2}\left(\frac{x}{\pi \lambda}\right)^{2} \text { for } x \in[0, \pi \lambda], \\
& V(x)=V_{0}-\frac{V_{0}}{2}\left(\frac{x}{\pi \lambda}-2\right)^{2} \text { for } x \in(\pi \lambda, 2 \pi \lambda],
\end{aligned}
$$


while the corresponding pinning force is given by

$$
\begin{gathered}
f_{\text {pin }}(x)=\frac{V_{0}}{\pi^{2} \lambda^{2}} x \text { for } x \in[0, \pi \lambda], \\
f_{\text {pin }}(x)=\frac{V_{0}}{\pi^{2} \lambda^{2}}(2 \pi \lambda-x) \text { for } x \in(\pi \lambda, 2 \pi \lambda],
\end{gathered}
$$

where $V_{0}=2 \pi A / R$, and both the approximated pinning force and pinning barrier are plot as red dot-dashed lines in Fig. 4.4 .4 (a) and (b), respectively. Good agreement has been achieved between calculated and approximated function forms, so that the DW motion behaviors can be analyzed based on Eqs. (4.4.8) and (4.4.9) by solving the following equations of motion derived from Eq. (4.4.1) with only STTs taken into account

$$
\begin{aligned}
& \frac{\dot{x}}{\lambda}-\alpha \dot{\varphi}=\frac{\gamma K_{\perp}}{M_{s}} \sin 2 \varphi+\frac{u}{\lambda} \\
& \alpha \frac{\dot{x}}{\lambda}+\dot{\varphi}=\beta \frac{u}{\lambda}-\frac{\gamma}{2 M_{s}} f_{p i n},
\end{aligned}
$$

where $K_{\perp}=K_{z}-K_{\sigma}=Q K$ with $Q=K_{\perp} / K=8$.

Two main cases are considered to calculate the relevant threshold current. For the depinning case, the DW is pinned by an energy barrier in the initial state, and then depinned by a current pulse with amplitude of $u$, i.e., the critical current $u_{c}$ to depin a motionless DW will be calculated, which usually happens in the racetrack memory process [21] implemented in zigzag nanowires. For the transportation case, the DW is moving steadily under constant current before transporting the barrier, i.e., the critical current $u_{c}$ to transport a steady motion DW will be calculated, which usually happens in nanowire circuits of DW based devices. Since our aforementioned assumptions require 
that the corner radius $R$ shouldn't be too small, we only consider corners with $R \geq 4 \lambda$ which indicates a relatively weak pinning regime and then threshold currents at Regime I [61] with very small $\varphi$ will be mainly calculated but threshold values at Regime II (intermediate pinning regime) [61] will be also briefly discussed.

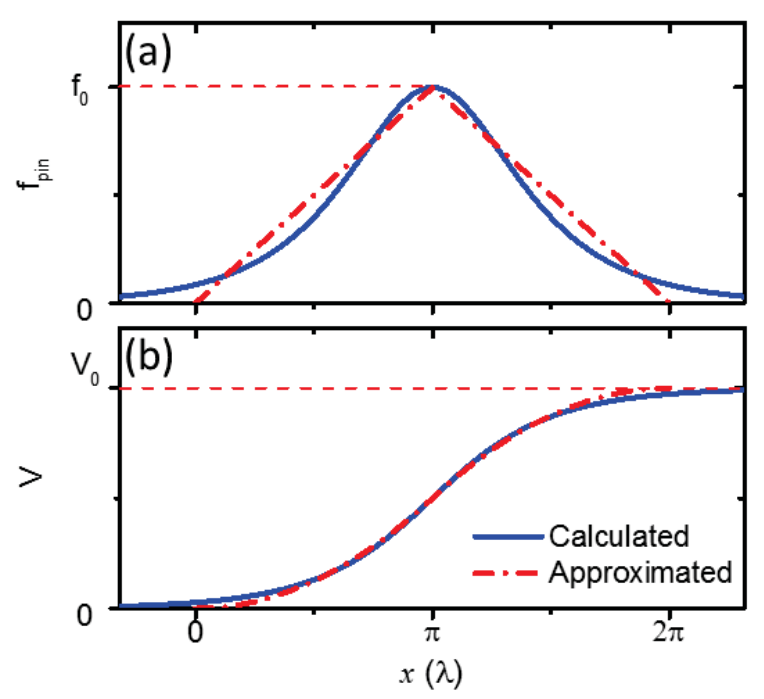

Figure 4.4.4 Calculated (solid blue lines, derived from Eq. (4.4.7)) and approximated (dot-dashed red lines, described by Eq. (4.4.8) and (4.4.9)) profiles of (a) pinning force and (b) pinning potential.

For the depinning case, at Regime I for $x \in[0, \pi \lambda]$, a simple equation can be obtained from Eq. (4.4.10),

$$
\left(1+\alpha^{2}\right) \ddot{x}+\frac{1}{\tau} \dot{x}+\omega^{2} x=2 \beta \tilde{u}
$$

where $\frac{1}{\tau}=\frac{2 \alpha \gamma K_{\perp}}{M_{s}}\left(1+\frac{V_{0}}{4 \pi^{2} \lambda K_{\perp}}\right) \approx \frac{2 \alpha \gamma K_{\perp}}{M_{s}}, \omega^{2}=\frac{\gamma^{2} V_{0} K_{\perp}}{\pi^{2} \lambda M_{s}^{2}}$, and $\tilde{u}=\frac{\gamma K_{\perp}}{M_{s}} u$. With the initial condition $x(x)=0$ and $\dot{x}(x)=u$, Eq. (4.4.11) can be solved and the solution is given by 


$$
x(t)=\frac{2 \beta \tilde{u}}{\omega^{2}}\left[1-e^{-\frac{t}{2 \tau}}\left(\cos \omega^{\prime} t+\frac{1}{2 \omega^{\prime} \tau} \sin \omega^{\prime} t\right)\right]+\frac{u}{\omega^{\prime}} e^{-\frac{t}{2 \tau}} \sin \omega^{\prime} t
$$

where $\omega^{\prime}=\sqrt{\omega^{2}-1 / 4 \tau^{2}}$. Let's assume a weak damping limit, which requires $\omega \tau=\beta_{c} / \alpha>>1$ where $\beta_{c}=\frac{1}{\sqrt{2 \pi Q}} \sqrt{\frac{\lambda}{R}}$, and then $\omega^{\prime} \approx \omega$. Under that limit, for $\beta>>\beta_{c}$ (Regime I-a), we have

$$
\begin{gathered}
x=\frac{2 \beta \tilde{u}}{\omega^{2}}\left(1-\cos \theta_{a}\right), \\
\dot{x}=\frac{2 \beta \tilde{u}}{\omega} \sin \theta_{a} ;
\end{gathered}
$$

while for $\beta<\beta_{c}$ (Regime I-b), we have

$$
\begin{aligned}
& x=\frac{u}{\omega} \sin \theta_{b}, \\
& \dot{x}=u \cos \theta_{b} .
\end{aligned}
$$

When $x \in(\pi \lambda, 2 \pi \lambda]$, Eq. (4.4.11) is replaced by

$$
\left(1+\alpha^{2}\right) \ddot{x}+\frac{1}{\tau} \ddot{x}-\omega^{2}(x-2 \pi \lambda)=2 \beta \ddot{u}
$$

and the corresponding solution under the weak damping limit is also modified by

$$
x(t) \approx 2 \pi \lambda-\frac{2 \beta \tilde{u}}{\omega^{2}}+A e^{-\omega t}+B e^{\omega t} .
$$

At Regime I-a, the initial conditions satisfy

$$
\begin{gathered}
x(0)=2 \pi \lambda-\frac{2 \beta \tilde{u}}{\omega^{2}}+A+B=\frac{2 \beta \tilde{u}}{\omega^{2}}\left(1-\cos \theta_{a}\right)=\pi \lambda, \\
\hat{x}(0)=\omega(B-A)=\frac{2 \beta \tilde{u}}{\omega} \sin \theta_{a} .
\end{gathered}
$$


The only required condition for DW depinning to occur (i.e., $x>2 \pi \lambda$ ) is $B>0$ which requires $\cos \theta_{a}>-1 / \sqrt{2}$, and therefore the critical current at Regime I-a is

$$
u_{c}^{I-a}=\frac{\gamma V_{0}}{2 \pi(1+1 / \sqrt{2}) \beta M_{s}}=\frac{\gamma A}{(1+1 / \sqrt{2}) M_{s} \beta R}
$$

Similarly, the critical current at Regime I-b is

$$
u_{c}^{I-b}=\frac{\gamma \sqrt{\lambda V_{0} K_{\perp}}}{M_{s}} f\left(\frac{\beta}{\beta_{c}}\right)=\frac{\gamma \sqrt{2 \pi A \lambda K_{\perp}}}{M_{s} \sqrt{R}} f\left(\frac{\beta}{\beta_{c}}\right) \text {. }
$$

Where $f\left(\frac{\beta}{\beta_{c}}\right)=\frac{2}{\beta / \beta_{c}+\sqrt{2-\left(\beta / \beta_{c}\right)^{2}}}$ and for $\beta<<\beta_{c}, f \rightarrow \sqrt{2}$, so that Eq. (4.4.22) will be

$$
u_{c}^{I-b} \rightarrow \frac{2 \gamma \sqrt{\pi A \lambda K_{\perp}}}{M_{s} \sqrt{R}}
$$

It is noted that for $\beta>\beta_{c}$, the threshold current $u_{c}^{I} \rightarrow u_{c}^{I-a} \propto(\beta R)^{-1}$ which is dependent of $\beta$ and has a relatively small value due to large $\beta$, while for $\beta<<\beta_{c}$, $u_{c}^{I} \rightarrow u_{c}^{I-b} \propto R^{-1 / 2}$ which is weakly dependent of $\beta$ and has a relatively large value due to the tiny $\beta$, and the main characteristics manifested by those conclusions are in a good agreement with Ref. [61] although the pinning effects are different. For arbitrary $\beta$, the threshold current steers between $u_{c}^{I-a}$ and $u_{c}^{I-b}$. Fig. 4.4 .5 (a) shows the threshold currents $u_{c}$ vs. $(R / \lambda)^{-1}$ under different values of $\beta$ for the depinning case. Solid lines are calculated results according to Eq. (4.4.21) and (4.4.23), while dot-dashed lines are numerically calculated results based on the 1D model according to Eq. (4.4.10). It is clear 
that cases of large $\beta$ fall into Regime I-a while cases of small $\beta$ fall into Regime I-b as stated above. It is noted that for $\lambda / R$ up to 0.25 , almost all that cases are in Regime I except the case $\beta=0.01<\alpha$ for which the depinning process happens in Regime II for $R<5.7 \lambda$. At Regime II, the threshold current can be determined by

$$
u_{c}^{I I}=\frac{\gamma \lambda K_{\perp}}{M_{s}},
$$

which is $\sim 600 \mathrm{~m} / \mathrm{s}$ for the depinning case and independent of both $R$ and $\beta$, consistent with our numerical results shown in Fig. 4.4.5 (a). Unlike Regime I, once the depinning process is happened at Regime II, the DW will be flipped and its chirality will also be reversed.

Actually, the above calculations for the depinning case are based on the absolutely abrupt current pulse with the rising edge jumping from 0 to $u$ directly. However, once the time window for the rising edge is relatively large, lager than $\sim 1 \mathrm{~ns}$ for instance, since the DW can response the stimuli very fast, the inertia effect disappears, and the threshold current will be determined by the pinning force maximum, which is given by

$$
u_{c}^{I}=\frac{\gamma A}{M_{s} \beta R}=(1+1 / \sqrt{2}) u_{c}^{I-a}
$$

Eq. (4.4.25) indicates that for the current pulse with slowly rising edge, the threshold depinning current at Regime I only depends on $\beta$ and $R$ in a linear relationship just like at Regime I-a, and Regime I-b is omitted for lacking of inertia effect. 

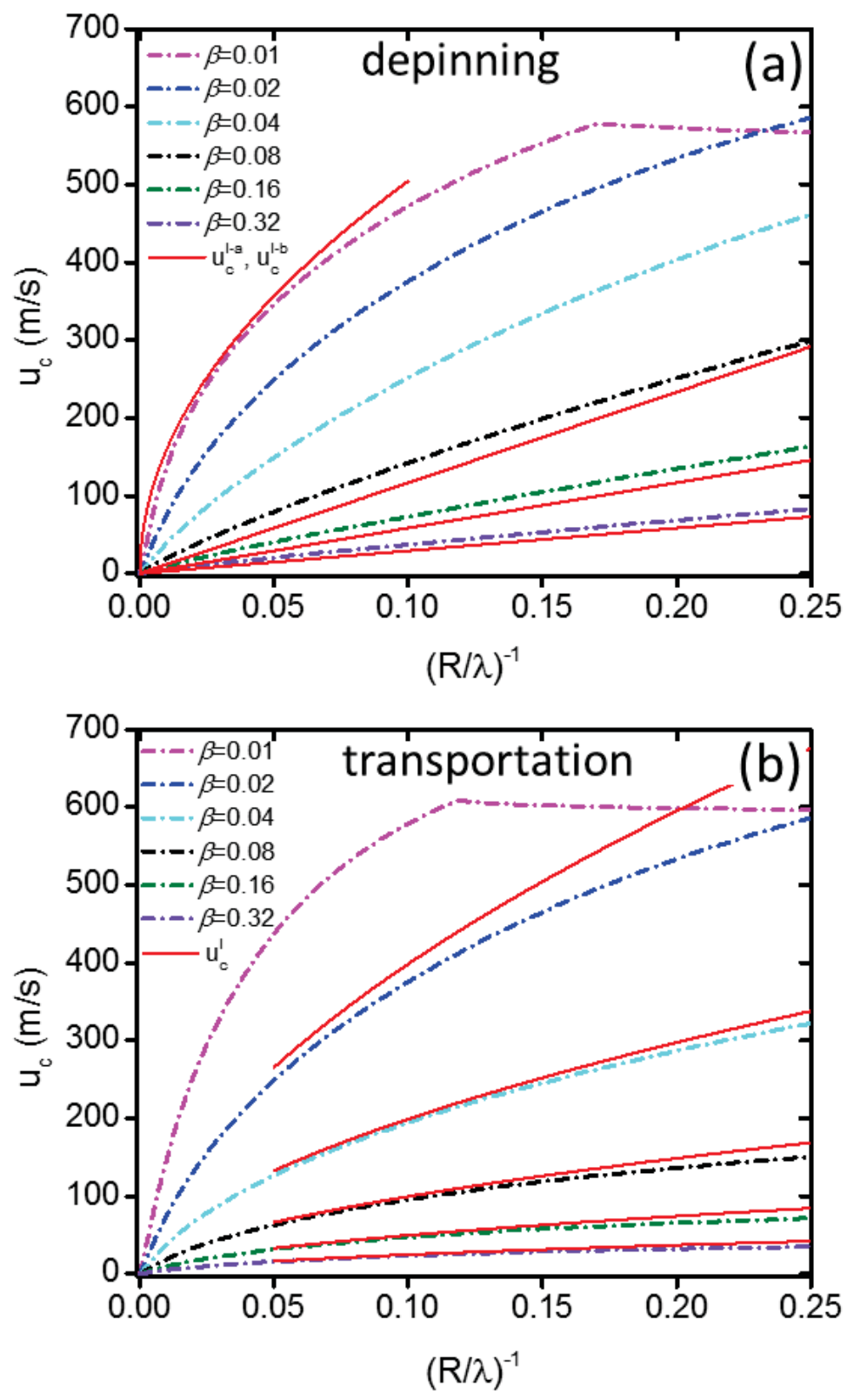

Figure 4.4.5 The threshold currents $u_{c}$ vs. $(R / \lambda)^{-1}$ under different values of $\beta$ for (a) the depinning case and (b) the transportation case induced by STTs. Red solid lines represent the calculated results according to Eq. (4.4.21), (4.4.23) and (4.4.29), while dot-dashed lines are numerically calculated results from the 1D model according to Eq. (4.4.10). 
For the transportation case, at Regime I, the only difference from the above depinning case is the initial DW velocity which is the steady motion velocity $\dot{x}(0)=u \beta / \alpha$, and then the solution becomes

$$
x(t)=\frac{2 \beta u}{\omega^{2}}\left[1-e^{-\frac{t}{2 \tau}}\left(\cos \omega^{\prime} t+\frac{1}{2 \omega^{\prime} \tau} \sin \omega^{\prime} t\right)\right]+\frac{\beta u}{\alpha \omega^{\prime}} e^{-\frac{t}{2 \tau}} \sin \omega^{\prime} t
$$

Under the weak damping limit, the second term is dominated, and for $x \in[0, \pi \lambda]$ we have

$$
\begin{aligned}
& x=\frac{\beta u}{\alpha \omega} \sin \theta, \\
& \dot{x}=\frac{\beta}{\alpha} u \cos \theta .
\end{aligned}
$$

When $x \in(\pi \lambda, 2 \pi \lambda]$, the motion of DW is described by Eq. (4.4.18), and the threshold current is therefore given by

$$
u_{c}^{I}=\frac{\alpha}{\beta} \frac{\gamma \sqrt{\lambda V_{0} K_{\perp}}}{M_{s}} f\left(\frac{\alpha}{\beta_{c}}\right)=\frac{\alpha}{\beta} \frac{\gamma \sqrt{2 \pi A \lambda K_{\perp}}}{M_{s} \sqrt{R}} f\left(\frac{\alpha}{\beta_{c}}\right)
$$

where $f\left(\frac{\alpha}{\beta_{c}}\right)=\frac{2}{\alpha / \beta_{c}+\sqrt{2-\left(\alpha / \beta_{c}\right)^{2}}} \rightarrow \sqrt{2}$ at weak damping limit. Fig. 4.4 .5

shows the threshold currents under different values of $\beta$ for the transportation case. Good agreement between the 1D numerical results and the calculated results described by Eq. (4.4.29) has been achieved for cases of $\beta \geq \alpha$. For $\beta=0.01$, Regime II begins to emerge for $R<8.5 \lambda$. The threshold current at Regime II is also described by Eq. (4.4.24). It is noted from Fig. 4.4.5 that the critical currents for the transportation case are always lower than those of the depinning case when $\beta>\alpha$, otherwise they are higher 
when $\beta<\alpha$, and both cases share the same value when $\beta=\alpha$, all of which are determined by the initial velocity.

As for the steady motion of DW at Regime I for the transportation case, however, there is an additional critical value given by

$$
u_{\max }=\left|\frac{\alpha}{\beta-\alpha}\right| \frac{\gamma \lambda K_{\perp}}{M_{s}},
$$

beyond which the steady motion will be destroyed, i.e., the following condition

$$
\frac{u_{c}^{I}}{u_{\max }}=2 \pi\left|1-\frac{\alpha}{\beta}\right| \beta_{c} f\left(\frac{\alpha}{\beta_{d}}\right)<1
$$

should be satisfied to make sure Regime I is existed for the transportation case. It can be expected that $\beta<<\alpha$ is not favored by Regime I steady transportation process.

The velocity for the DW that just escapes the barrier (i.e., $x=2 \pi \lambda$ ) under threshold current for both depinning and transportation cases can be calculated from Eq. (4.4.18) in the conditions of $B<<1$ and $\omega t>>1$,

$$
v_{e s}=u_{c} \beta / \beta_{c},
$$

where $u_{c}$ represents the corresponding threshold current described by Eq. (4.4.21), (4.4.22), (4.4.25) or (4.4.29) at Regime I. For the depinning case, $v_{e s} \propto R^{-1 / 2}$ at Regime Ia, while $v_{e s} \propto \beta$ at Regime I-b. For the transportation case, $v_{e s} \rightarrow$ cons. at weak damping limit.

\subsubsection{Spin-orbit Torques}

\subsection{Rashba Effect}


The current induced DW motion for both depinning and transportation cases will be affected in some certain manner once spin-orbit torques are taken into account. RE can contribute an effective in-plane transverse field perpendicular to the current direction as described by Eq. (4.4.2), and such a field will definitely change the magnetization structure as well as the relevant pinning effect. For small $\varphi$, the DW structure can be described by

$$
\theta^{\prime}=\left(\sin \theta-\sin \theta_{0}\right) / \lambda
$$

where $\sin \theta_{0} \approx M_{s} H_{R} / 2 K$ and $\theta_{0}$ represents the tilt angle of magnetization at domain state induced by the Rashba effective field. For relative small RE, i.e., $\theta_{0}$ is small, we have $\sin \theta_{0} \approx \theta_{0}$, which corresponds to a relative small Rashba parameter $\alpha_{R}<10^{-12} e V m$. Since the Rashba effective field is proportional to the current according to Eq. (4.4.2), the RE induced tilt angle can be described by $\theta_{0}=\kappa_{R} u$, where the Rashba effective coefficient $\kappa_{R}$ is given by

$$
\kappa_{R}=\frac{M_{s} e \alpha_{R}}{g \mu_{B} K},
$$

which can be either positive or negative depending on the layout of SO layer and oxide layer as well as the DW types, and hence both the positive and negative values of $\kappa_{R}$ or $\theta_{0}$ will be considered in this section. Fig. 4.4.6 (a) shows the energy barrier profiles under RE with different values of $\theta_{0}$. For positive $\theta_{0}$, the barrier height is reduced, while for negative $\theta_{0}$, the barrier is even higher. As is expected, the sign of $\theta_{0}$ doesn't depend on DW chirality, it depends on the magnetization direction of DW: if the DW 
magnetization direction is the same with Rashba effective field, $\theta_{0}$ will be positive; otherwise, negative. Take an $180^{\circ} \mathrm{DW}$ for example, when the Rashba field is applied in the same direction of DW magnetization, the rotation angle will be smaller than $180^{\circ}$, resulting in a reduced barrier, where an ultimate case is achieved when the field is so huge that $\theta_{0} \rightarrow 90^{\circ}$ and DW barrier disappears; when the Rashba field applied in the opposite direction, the rotation angle will be larger than $180^{\circ}$, resulting in higher barrier, and when the field is large enough the DW will be flipped.

The pinning barrier height under $\mathrm{RE}$ is $\left(1-2 \theta_{0} / \pi\right) V_{0}$ and the corresponding maximum pinning force is $\left(1-\theta_{0}\right) f_{0}$. In the same approximation made in Sec. IIIA, the pinning force will have the following form

$$
\begin{aligned}
& f_{\text {pinR }}(x)=\frac{V_{R}}{\pi^{2} \lambda^{2}} x \text { for } x \in[0, \pi \lambda(1+\delta)], \\
& f_{\text {pinR }}(x)=\frac{V_{R}}{\pi^{2} \lambda^{2}}[2 \pi \lambda(1+\delta)-x] \text { for } x \in(\pi \lambda(1+\delta), 2 \pi \lambda(1+\delta)],
\end{aligned}
$$

where $\delta=(1-2 / \pi) \theta_{0}$, and $V_{R}=2 \pi A R^{-1}\left[1-(2-2 / \pi) \theta_{0}\right]$ is the Rashba effective pinning barrier height. The pinning force approximated according to Eq. (4.4.35) is shown in Fig. 4.4.6 (b). The equations of motion derived from Eq. (4.4.1) with RE taken into account are given by

$$
\begin{gathered}
\frac{\dot{x}}{\lambda}-\alpha \frac{\dot{\varphi}}{1-\theta_{0}}=\frac{\gamma K_{\perp R}}{\left(1-\theta_{0}\right) M_{s}} \sin 2 \varphi+\frac{u}{\lambda} \\
\alpha \frac{\dot{x}}{\lambda}+\frac{\dot{\varphi}}{1-\theta_{0}}=\beta \frac{u}{\lambda}-\frac{\gamma}{2 M_{s}} f_{\text {pinR }} .
\end{gathered}
$$


where $K_{\perp R}=K_{\perp}\left(1+Q^{-1} \theta_{0} / \cos \varphi\right) \approx K_{\perp}\left(1+Q^{-1} \theta_{0}\right)$, and actually, since $Q$ is large and $\theta_{0}$ is small, the difference between $K_{\perp}$ and $K_{\perp R}$ can be ignored in our following cases.
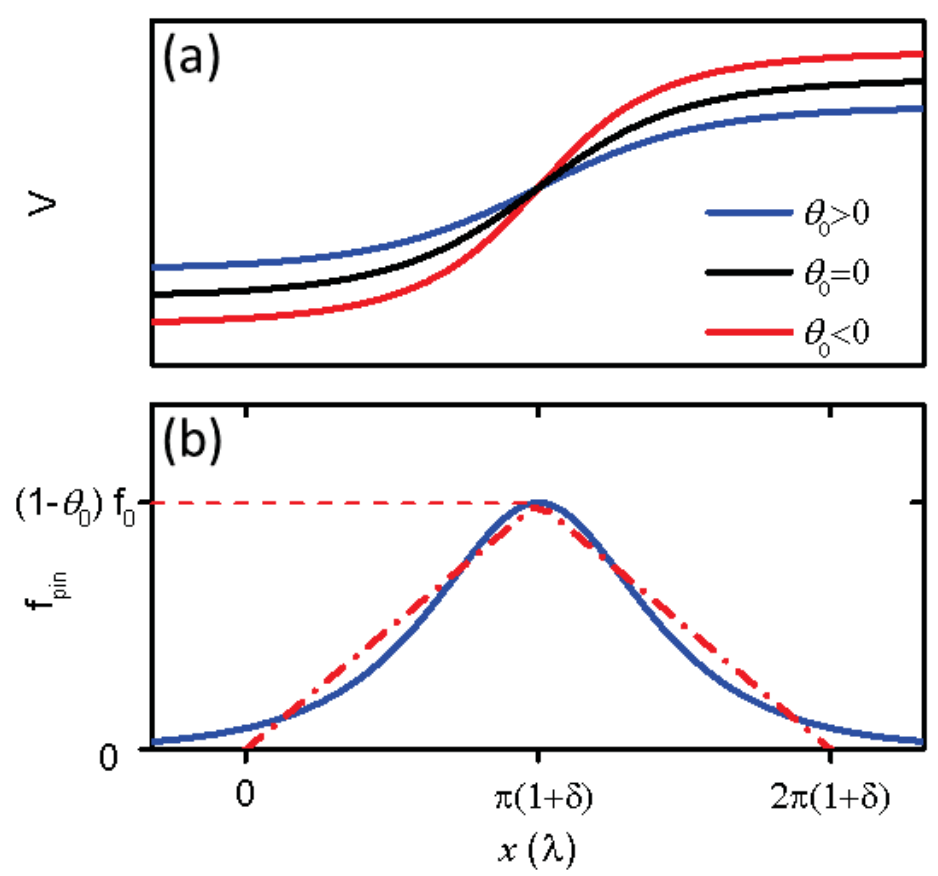

Figure 4.4.6 (a) Calculated pinning potential profiles for $\theta_{0}>0, \theta_{0}=0$ and $\theta_{0}<0$. (b) Calculated (solid blue line) and approximated (dot-dashed red line) profiles for the pinning force under RE.

The DW depinning and transportation by RE can also be analyzed in the similar procedure as made in Sec. IIIA. For both depinning and transportation cases, the DW motion at Regime I with $x \in[0, \pi \lambda(1+\delta)]$ can also be described by Eq. (4.4.11) with only replacing $K_{\perp}$ and $V_{0}$ by $K_{\perp R}$ and $V_{R}$, respectively, while for $x \in(\pi \lambda(1+\delta), 2 \pi \lambda(1+\delta)]$, the equation will be

$$
\left(1+\alpha^{2}\right) \ddot{x}+\frac{1}{\tau} \dot{x}-\omega^{2}[x-2 \pi \lambda(1+\delta)]=2 \beta \tilde{u},
$$


which is quite similar with Eq. (4.4.17). By solving both Eq. (4.4.11) and (4.4.37) with corresponding initial conditions, threshold currents for both cases can be calculated.

For the depinning case, the threshold current at Regime I-a and Regime I-b at weak damping limit are given by

$$
\begin{gathered}
u_{c R}^{I-a}=\frac{\gamma A\left(1-\theta_{0}\right)}{(1+1 / \sqrt{2}) M_{s} \beta R}, \\
u_{c R}^{I-b}=\frac{\gamma \sqrt{2 \pi A \lambda K_{\perp}}}{M_{s} \sqrt{R}} f\left(\frac{\beta}{\beta_{c}}\right)\left[1-\left(1 / \pi-0.5 Q^{-1}\right) \theta_{0}\right] .
\end{gathered}
$$

Considering $\theta_{0}$ is dependent of current by $\theta_{0}=\kappa_{R} u$, Eq. (4.4.38) and (4.4.39) should be rewritten in the following forms

$$
\begin{gathered}
u_{c R}^{I-a}=\frac{u_{c}^{I-a}}{1+\kappa_{R} u_{c}^{I-a}}, \\
u_{c R}^{I-b}=\frac{u_{c}^{I-b}}{1+\left(1 / \pi-0.5 Q^{-1}\right) \kappa_{R} u_{c}^{I-b}},
\end{gathered}
$$

where $u_{c}^{I-a}$ and $u_{c}^{I-b}$ are threshold currents for STT cases in Sec. IIIA, which are described by Eq. (4.4.21) and (4.4.22), respectively. Fig. 4.4 .7 (a) shows the critical currents for depinning case by $\mathrm{RE}$ with different values of $\kappa_{R}$ at $\beta=0.08$ which roughly falls into Regime I-a as noted in Fig. 4.4 .5 (a). For positive $\kappa_{R}$, those critical values are reduced compared to the STT case of $\kappa_{R}=0$, and larger $\kappa_{R}$ will result in smaller $u_{c R}$. The calculation results at Regime I-a described by Eq. (4.4.40) are shown by solid lines in Fig. 4.4.7 (a), which manifests the same trend with the numerical results. For negative $\kappa_{R}$, the critical values are higher than the STT case of $\kappa_{R}=0$ as expected, but the values 
for larger $\kappa_{R}$ (for example, $\kappa_{R}=-0.001$ ) are somewhat depressed instead, which seems due to the reduced threshold current at Regime II (relative large $\varphi$ ) given by

$$
u_{c R}^{I I}=\frac{u_{c}^{I I}}{1-\left(1+Q^{-1}\right) \kappa_{R} u_{c}^{I I}},
$$

which is $\sim 350 \mathrm{~m} / \mathrm{s}$ for $\kappa_{R}=-0.001$ and such a kind of depression can be also found in STT cases when the critical current is close to Regime II. It can be briefly concluded for the depinning case that RE can effectively reduce the threshold current for positive $\kappa_{R}$, but it is difficult to increase the threshold value for negative $\kappa_{R}$ due to the depression of Regime II.

For the transportation case, the threshold current at Regime I under weak damping limit can be given by

$$
u_{c R}^{I}=\frac{u_{c}^{I}}{1+\left(1 / \pi-0.5 Q^{-1}\right) \kappa_{R} u_{c}^{I}}
$$

where $u_{c}^{I}$ is the threshold current for STT case in Sec. IIIA described by Eq. (4.4.29). Both the calculation (Eq. (4.4.43)) and numerical results for this transportation case by RE with different values of $\kappa_{R}$ at $\beta=0.08$ are shown in Fig. 4.4 .7 (b). Although the critical values as well as their deviation amplitude due to RE for the transportation case are smaller than that for the depinning case as expected for $\beta>\alpha$, the general trend for both cases is similar. For positive $\kappa_{R}$, those critical values are smaller than the STT case, while for negative $\kappa_{R}$, the critical values are depressed especially for large pinning barrier. 

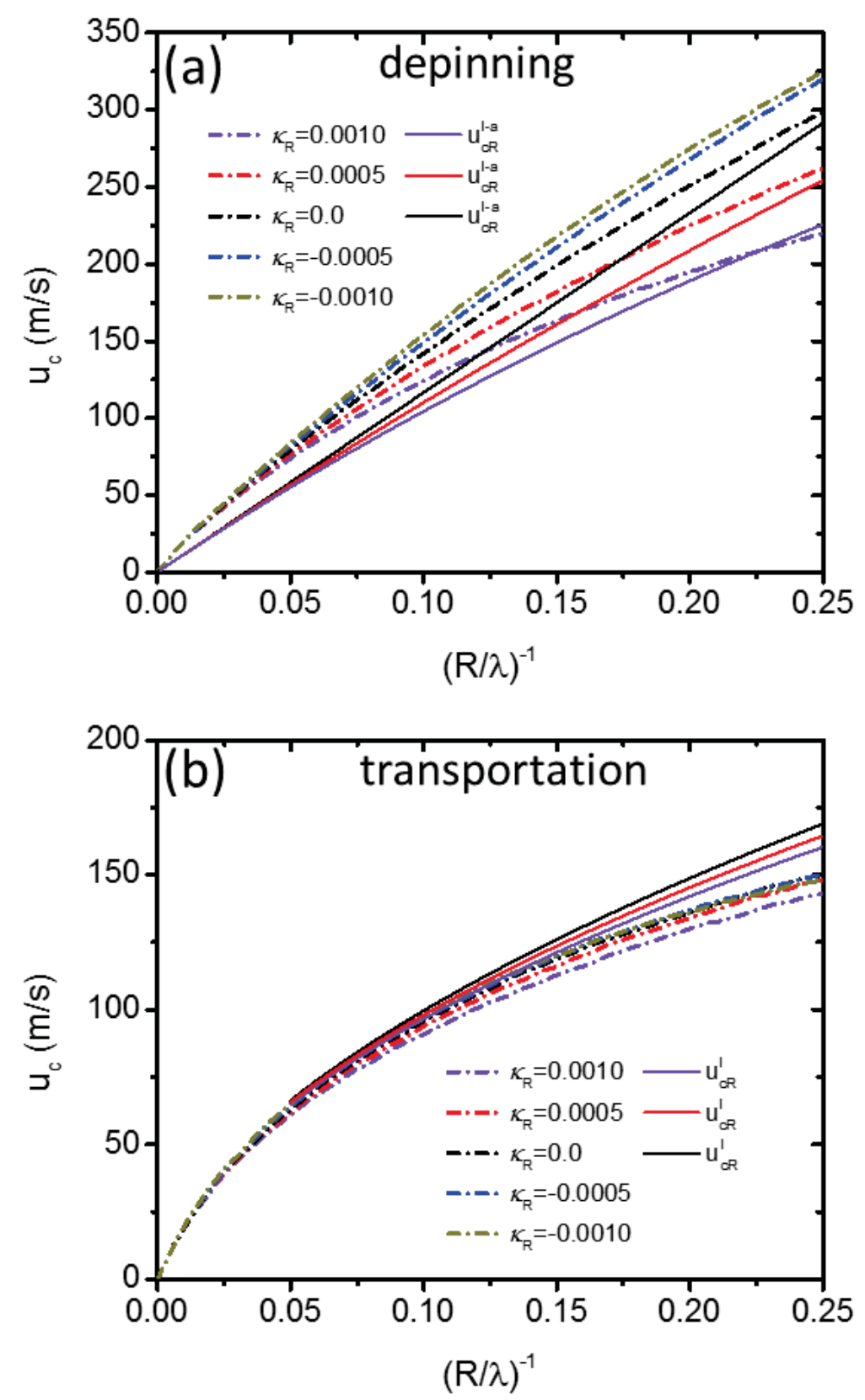

Figure 4.4.7 The threshold currents $u_{c}$ vs. $(R / \lambda)^{-1}$ under different values of Rashba effective coefficient $\kappa_{R}$ at $\beta=0.08$ for (a) the depinning case and (b) the transportation case induced by RE. Solid lines represent the calculated results according to Eq. (4.4.40) and (4.4.43), while dotdashed lines are numerically calculated results from the 1D model according to Eq. (4.4.36). 
Based on the above results which only consider weak RE, general conclusions with strong RE (for example, $\alpha_{R}>10^{-11} \mathrm{eVm}$ or $\left|\kappa_{R}\right| \sim 0.1$ ) can also be predicted: for positive $\kappa_{R}$, both the pinning barrier and the threshold current will be significantly reduced, and the process for depinning or transportation will take place at Regime I, since the threshold current at Regime II is significantly increased; for negative $\kappa_{R}$, the depinning or transportation process usually happens at Regime II and still with a drastically reduced critical current, but the DW will be flipped.

\subsection{Spin-Hall Effect}

Unlike RE which manifests a field-like effect, the SHE rather modifies the damping effect, and hence the energy barrier as well as the corresponding pinning force will be exactly the same with the STT case described by Eq. (4.4.8) and (4.4.9). Therefore, the equations of motion of DW derived from Eq. (4.4.1) with SHE taken into account will be

$$
\begin{gathered}
\frac{\dot{x}}{\lambda}-\alpha \dot{\varphi}=\frac{\gamma K_{\perp}}{M_{s}} \sin 2 \varphi+\frac{u}{\lambda} \\
\alpha \frac{\dot{x}}{\lambda}+\dot{\varphi}=\beta \frac{u}{\lambda}-\alpha_{S H} \sin \varphi-\frac{\gamma}{2 M_{s}} f_{p i n} .
\end{gathered}
$$

In this section, both the depinning and the transportation cases are also taken into account for the calculation of threshold currents induced by SHE.

For the depinning case, at Regime I, for $x \in[0, \pi \lambda]$, a simple equation similar with Eq. (4.4.11) can be obtained from Eq. (4.4.44), 


$$
\left(1+\alpha^{2}\right) \ddot{x}+\frac{1}{\tau_{S H}} \ddot{x}+\omega^{2} x=2 \tilde{\beta} \ddot{u},
$$

where $\frac{1}{\tau_{S H}}=\alpha_{S H}+\frac{1}{\tau}=\frac{2 \ddot{\alpha} \gamma K_{\perp}}{M_{s}}$, and the SH induced effective parameters $\ddot{\alpha}$ and $\tilde{\beta}$ are given by

$$
\begin{gathered}
\tilde{\alpha}=\alpha+\tilde{\alpha}_{S H}, \\
\tilde{\beta}=\beta+\tilde{\alpha}_{S H},
\end{gathered}
$$

where $\ddot{\alpha}_{S H}=\frac{\alpha_{S H} M_{s}}{2 \gamma K_{\perp}}$ is denoted as the SH effective damping parameter correction. Since $\alpha_{S H}$ is proportional to the current according to Eq. (4.4.3), the damping correction can also be described by $\hat{\alpha}_{S H}=\kappa_{S H} u$ with coefficient $\kappa_{S H}$ that can also be either positive or negative depending on the SO layers (the thickness and material of the top and bottom layers) as well as the DW types, and hence both the positive and negative values of $\kappa_{S H}$ or $\tilde{\alpha}_{S H}$ will be considered in this section. For $x \in(\pi \lambda, 2 \pi \lambda]$, an equation similar with Eq. (4.4.17) is given by

$$
\left(1+\alpha^{2}\right) \tilde{x}+\frac{1}{\tau_{S H}} \tilde{x}-\omega^{2}(x-2 \pi \lambda)=2 \tilde{\beta} \ddot{u}
$$

For positive $\tilde{\alpha}_{S H}$ or $\kappa_{S H}$, by applying relevant initial conditions, critical currents at Regime I-a $\left(\ddot{\beta}>>\beta_{c}\right)$ and Regime I-b $\left(\ddot{\beta}<<\beta_{c}\right)$ under weak damping limit satisfying $\omega \tau_{S H}=\beta_{c} / \tilde{\alpha}>>1$ can be obtained by solving Eq. (4.4.45) and (4.4.48) in the same procedure stated in Sec. IIIA, which are given by 


$$
\begin{aligned}
& u_{c S H}^{I-a}=\frac{\gamma A}{(1+1 / \sqrt{2}) M_{s} \ddot{\beta} R}=\frac{\beta}{\tilde{\beta}} u_{c}^{I-a}, \\
& u_{c S H}^{I-b}=\frac{\gamma \sqrt{2 \pi A \lambda K_{\perp}}}{M_{s} \sqrt{R}} f\left(\frac{\tilde{\beta}}{\beta_{c}}\right) \sim u_{c}^{I-b} .
\end{aligned}
$$

Considering $\tilde{\alpha}_{S H}$ is dependent of current by $\tilde{\alpha}_{S H}=\kappa_{S H} u$, Eq. (4.4.49) should be rewritten in the following form

$$
u_{c S H}^{I-a}=\frac{1}{2 \kappa_{S H}}\left[\sqrt{\beta^{2}+\frac{4 \gamma A \kappa_{S H}}{(1+1 / \sqrt{2}) M_{s} R}}-\beta\right] .
$$

For negative $\tilde{\alpha}_{S H}$ or $\kappa_{S H}$, there is a special regime, denoted as Regime I-c, which depends on the sign of $\tau_{S H}$. According to the solution of Eq. (4.4.45),

$$
x(t)=\frac{2 \ddot{\beta} \ddot{u}}{\omega^{2}}\left[1-e^{-\frac{t}{2 \tau_{S H}}}\left(\cos \omega^{\prime} t+\frac{1}{2 \omega^{\prime} \tau_{S H}} \sin \omega^{\prime} t\right)\right]+\frac{u}{\omega^{\prime}} e^{-\frac{t}{2 \tau_{S H}}} \sin \omega^{\prime} t,
$$

once $\tau_{S H}$ becomes negative, the oscillation amplitude of DW velocity $\dot{x}(t)$ will be dominated by the divergent exponential function, i.e., the velocity can become larger and larger until it is large enough to depin from the pinning barrier although the current might be much smaller than the required depinning value for the STT case. In another word, it is $\tau_{S H}=0$ or $\tilde{\alpha}=0$ that determines the critical value at Regime I-c which is given by

$$
u_{c S H}^{I-c}=-\frac{\alpha}{\kappa_{S H}} .
$$

Both the numerical and calculation results at Regime I-c agree with each other very well as shown in Fig. 4.4 .8 (a) for negative $\kappa_{S H}$. It is noted that SHE with negative $\kappa_{S H}$ can 
effectively reduce the depinning current especially for large $\kappa_{S H}$ and small $\alpha$ as expected by Eq. (4.4.53), and the DW will not be flipped during the depinning process at Regime I-c. But for very long current pulse, the DW will be flipped after depinning from the barrier, and then $\kappa_{S H}$ becomes positive resulting in a steady motion of DW. It is also noted from Fig. 4.4.8 (a) that for positive $\kappa_{S H}$, the threshold currents are also reduced due to the increased parameter $\tilde{\beta}$, which can be roughly described by Eq. (4.4.49) or (4.4.51). Both equations that obtained under weak damping limit, however, underestimate the threshold currents. Actually, positive $\kappa_{S H}$ might result in a very large effective damping parameter $\tilde{\alpha}$, which can break the weak damping limit so that the attenuating exponential function should be considered and then larger current is required for the depinning process to occur, and that is why the numerical results are larger than our calculated results described by Eq. (4.4.51).

For the transportation case, at Regime I, the DW motion behavior can be obtained by solving Eq. (4.4.45) using a different initial DW velocity, the steady motion velocity $\dot{x}(0)=u \tilde{\beta} / \tilde{\alpha}$, and then the solution is

$$
x(t)=\frac{2 \widetilde{\beta} \ddot{u}}{\omega^{2}}\left[1-e^{-\frac{t}{2 \tau_{S H}}}\left(\cos \omega^{\prime} t+\frac{1}{2 \omega^{\prime} \tau_{S H}} \sin \omega^{\prime} t\right)\right]+\frac{\tilde{\beta} u}{\tilde{\alpha} \omega^{\prime}} e^{-\frac{t}{2 \tau_{S H}}} \sin \omega^{\prime} t .
$$

Under the weak damping limit, the threshold current is therefore calculated in the same procedure stated in Sec. IIIA, which is given by

$$
u_{c S H}^{I}=\frac{\tilde{\alpha}}{\tilde{\beta}} \frac{\gamma \sqrt{2 \pi A \lambda K_{\perp}}}{M_{s} \sqrt{R}} f\left(\frac{\tilde{\alpha}}{\beta_{c}}\right) .
$$


where $f\left(\frac{\tilde{\alpha}}{\beta_{c}}\right) \rightarrow \sqrt{2}$ at weak damping limit. Considering $\tilde{\alpha}_{S H}=\kappa_{S H} u$, Eq. (4.4.55) is therefore given in the following form

$$
u_{c S H}^{I}=\frac{1}{2 \kappa_{S H}}\left[\sqrt{\left(\beta-\kappa_{S H} u_{c}^{I-b}\right)^{2}+4 \alpha \kappa_{S H} u_{c}^{I-b}}-\left(\beta-\kappa_{S H} u_{c}^{I-b}\right)\right],
$$

where $u_{c}^{I-b}$ is given by Eq. (4.4.23). Fig. 4.4 .5 (b) shows the threshold currents under different values of $\kappa_{S H}$ for the transportation case. Good agreement between the 1D numerical results and the calculated results described by Eq. (4.4.56) has been achieved for cases of negative $\kappa_{S H}$. The threshold currents are effectively reduced for negative $\kappa_{S H}$. For positive $\kappa_{S H}$, the threshold current is higher than the STT case, which can be roughly approximated by Eq. (4.4.55) but the weak damping limit is broken for large current. It is also noted that for very large $\kappa_{S H}$, the transportation case will coincide with the depinning case for relative large current, because the initial velocity for the transportation case approaches to the depinning case with large $\ddot{\alpha}$, i.e., $\dot{x}(0)=u \tilde{\beta} / \ddot{\alpha} \rightarrow u$

Unlike RE, the SHE will not modify the threshold current at Regime II for both depinning and transportation cases, but the maximum current to maintain steady motion of DW at Regime I for the transportation case is changed, which is given by

$$
u_{\max S H}=\left|\frac{\ddot{\alpha}}{\beta-\alpha}\right| \frac{\gamma \lambda K_{\perp}}{M_{s}}
$$


beyond which the steady motion will be destroyed. At Regime I-c for the depinning case where $\tilde{\alpha} \rightarrow 0$, and then we have $u_{\max S H} \rightarrow 0$ according to Eq. (4.4.57), that is why the DW will be flipped after depinning under a very long current pulse as mentioned above.
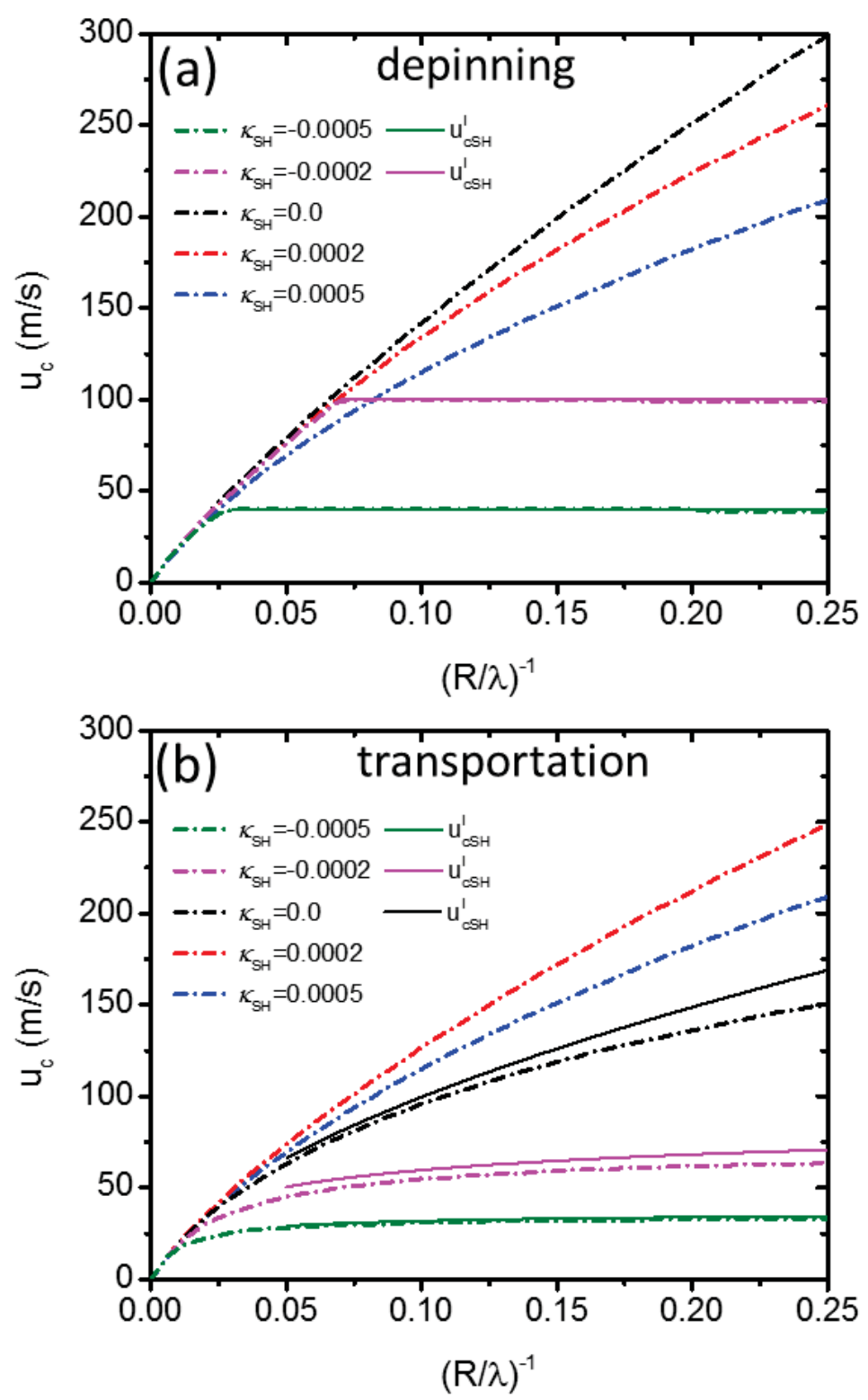

Figure 4.4.8 The threshold currents $u_{c}$ vs. $(R / \lambda)^{-1}$ under different values of SH effective coefficient $\kappa_{S H}$ at $\beta=0.08$ for (a) the depinning case and (b) the transportation case induced by SHE. Solid lines represent the calculated results according to Eq. (4.4.53) and (4.4.56), while dotdashed lines are numerically calculated results from the 1D model according to Eq. (4.4.44). 


\subsubsection{Conclusion}

In conclusion, current induced transverse DW depinning and transportation through circular geometry in planar nanowire by the conventional STTs and the interfacederived SOTs have been theoretically calculated, and those calculated results are also supported by our numerical results based on the 1D model. For STTs, both the depinning and transportation cases fall into Regime I for $\beta \geq 0.02=\alpha$ up to large pinning effect of $R=4 \lambda$ while partially fall into Regime II for $\beta<\alpha$. Under weak damping limit, the

threshold current is $u_{c}^{I-a} \propto(\beta R)^{-1}$ for $\beta>>\beta_{c}$ and $u_{c}^{I-b} \propto R^{-1 / 2}$ for $\beta<<\beta_{c}$ in the depinning case, while in the transportation case the threshold current is $u_{c}^{I} \propto \alpha \beta^{-1} R^{-1 / 2}$, and the threshold values for the latter case are smaller than the former one for $\beta>\alpha$ while larger for $\beta<\alpha$. For SOTs, the RE and SHE are considered separately for both depinning and transportation cases with $\beta=0.08=4 \alpha$. As for RE, the DW magnetization structure can be modulated by the Rashba effective transverse fiend and hence the pinning effect can be either reduced or increased depending on the sign of Rashba effective coefficient $\kappa_{R}$ which is determined by the interface and DW type. For positive $\kappa_{R}$, the pinning effect becomes smaller and then the threshold currents are reduced for both depinning and transportation cases; for negative $\kappa_{R}$, the pinning effect is larger, but the threshold currents are not evidently increased for both cases, because of the reduced threshold current at Regime II. As for SHE, the DW motion is modulated by the SH effective damping parameter correction $\tilde{\alpha}_{S H}$ which can also be either positive or negative depending on the sign of SH effective coefficient $\kappa_{S H}$. For negative $\kappa_{S H}$, the 
threshold currents are effectively reduced for both cases, and constant critical current $u_{c S H}^{I-c}=-\alpha / \kappa_{S H}$ exists at a special regime denoted as Regime I-c for the depinning case; for positive $\kappa_{S H}$, the threshold currents are also reduced for the depinning case due to the larger value of $\tilde{\beta}$, while for the transportation case, those values are larger than the STT transportation case, but will coincide with the SOT depinning case for large $\kappa_{S H}$. Our calculations for the SOTs demonstrate novel means to effectively reduce the threshold current and modulate the current induced DW depinning or transportation process. Since DW depinning and transportation processes through planar nanowire circular geometry are very important for magnetic memory and logic circuits, our work will shed a light on the developing of future DW-based devices. 


\subsection{Magnetic Vortex Racetrack Memory}

\subsubsection{Introduction}

Racetrack memory which is based on spin-polarized current induced domain wall (DW) motion was proposed in recent years in order to build up a novel non-volatile device with ultrahigh storage density [21]. The first demonstration of CMOS-integrated racetrack memory with a small capacity of 256 bits was reported by using Permalloy nanowires which has in-plane magnetic anisotropy (IMA) [219]. Recently, racetrack memory built on $\mathrm{CoFeB} / \mathrm{MgO}$ structures with perpendicular magnetic anisotropy (PMA) is also proposed with advantages of long data retention and smaller depinning current density [46, 220, 221]. For nanowires either with IMA or PMA, the information carrier is magnetic domain whose direction defines the bit instead of DW itself. Actually, a DW with modulatable freedom degrees such as the magnetization rotation sense or magnetization direction for transverse DWs and the core polarity for vortex DWs $[13,14$, $20,43,45,57,175,222]$ can also be treated as an information carrier. In particular, magnetic vortices, either in nanowires $[14,20]$ or in circular magnetic disks $[40-42,171$, $223,224]$, attract much more interest due to the nanosized vortex core $(10 \sim 20 \mathrm{~nm}$ diameter in Permalloy film), the distinct core polarity $(\mathrm{p}=+1$ for core magnetization pointing out of the vortex plane or $\mathrm{p}=-1$ pointing into the vortex plane), and the ultrafast core reversal dynamic by application of pulsed magnetic field [40-42, 171, 223, 224], alternating spin-polarized current [20,44], or even circularly polarized light [48]. All above unique properties of vortex demonstrate potential application for non-volatile memory with bits manifested by the core polarity. Due to the existence of such additional freedom degree (the vortex core polarity), as well as its movability driven by spin- 
polarized current, a combination of both racetrack memory and magnetic vortices, called magnetic vortex racetrack memory (VRM), can be proposed to not only provide a way to approach high vortex storage density, but also indicate different behaviors from DW racetrack memory (DWRM).

However, the novel VRM seems to require some other forms of vortices rather than the above two existent forms studied by most people, vortices either centered in isolated micro- or nano-disks or confined by notches in nanowires. It is because that 1) vortices in individual disks can't be transported, and 2) the vortex DWs in nanowires are easily transformed into transverse DWs under the stimuli of spin-polarized current [13, 45, 175], and 3) moreover, densely packed vortex DWs are easily to be annihilated. Therefore, a proper form of vortex that can't be removed or annihilated by current or densely packed neighbor vortices in a nanowire is required for VRM. As is known that densely packed domain stripes with obvious vortex structures through the cross section can be spontaneously formed in a thin film with weak perpendicular anisotropy [225], and then we can suppose that densely packed vortices may also be formed in nanowires with weak perpendicular anisotropy and such vortices may not be easily annihilated by current due to their lower energy. Thus, this paper employs phase field modeling and micromagnetic simulations to obtain densely packed vortices in the nanowire with weak perpendicular anisotropy at first, and then demonstrate two simple models of magnetic VRM to study current induced vortex motion behaviors in the nanowire to explore the unique properties of VRM.

\subsubsection{Micromagnetic Model}


In our micromagnetic model, the local magnetization is described by magnetization field $\mathbf{M}(\mathbf{r})=M_{s} \mathbf{m}(\mathbf{r}) \theta(\mathbf{r})$, where $M_{s}$ is the saturation magnetization, $\mathbf{m}(\mathbf{r})$ is a unit vector field for magnetization normalized by $M_{s}$, and $\theta(\mathbf{r})$ is shape function characterizing the geometry of the nanowires. $\theta(\mathbf{r})=1$ means inside magnetic material and 0 outside. The evolution of magnetization is described by the modified LandauLifshitz-Gilbert equation including spin transfer torque terms [51]

$$
\frac{\partial \mathbf{m}}{\partial t}=\gamma \mathbf{H}_{\mathrm{eff}} \times \mathbf{m}+\alpha \mathbf{m} \times \frac{\partial \mathbf{m}}{\partial t}-(\mathbf{u} \cdot \nabla) \mathbf{m}+\beta \mathbf{m} \times[(\mathbf{u} \cdot \nabla) \mathbf{m}]
$$

where $\mathbf{u}=\mathbf{J} P g \mu_{B} /\left(2 e M_{s}\right)$ is a vector along the direction of electrons motion, $J$ the current density, $P$ the polarization rate of current density, $\gamma$ the gyromagnetic constant, $\alpha$ the Gilbert damping constant, and $\mathbf{H}_{\text {eff }}$ the effective magnetic field or thermodynamic driving force $\mathbf{H}_{\mathrm{eff}}=-\delta E /\left(M_{s} \delta \mathbf{m}\right)$, the variational derivative of the free energy with respect to the magnetization field variable. The last two terms on the right are usually called adiabatic and nonadiabatic spin transfer torques, respectively $[3,5,8]$.

The total system free energy is a functional of $\mathbf{m}(\mathbf{r})$ and is evaluated as a sum of magnetic anisotropy energy, exchange energy, magnetostatic energy, and external magnetic energy [178], where the long-range magnetostatic energy is calculated in reciprocal space using the Fourier transform of the magnetization field [178]

$$
\begin{aligned}
E & =\int K_{u}\left\{1-[\mathbf{p}(\mathbf{r}) \cdot \mathbf{m}(\mathbf{r})]^{2}\right\} d^{3} r \\
& +\int A[\operatorname{grad} \mathbf{m}(\mathbf{r})]^{2} d^{3} r+\frac{1}{2} M_{s}^{2} \int \frac{d^{3} k}{(2 \pi)^{3}} 4 \pi \frac{|\mathbf{k} \cdot \mathbf{m}(\mathbf{k})|^{2}}{k^{2}} \\
& -M_{s}^{2} \int \mathbf{h}_{\text {ext }} \cdot \mathbf{m}(\mathbf{r}) d^{3} r
\end{aligned}
$$


where $K_{u}$ is the magnetocrystalline anisotropy constant, $\mathbf{p}(\mathbf{r})$ the magnetic easy direction, $A$ the exchange stiffness constant, and $\mathbf{h}_{e x t}$ the external magnetic field.

In this paper, parameters of Co bulk material are used in our simulations for its relative weak anisotropic constant. These parameters are: $M_{s}=1400 \mathrm{emu} / \mathrm{cm}^{3}$, magnetocrystalline anisotropy constant $K_{u}=5.2 \times 10^{6} \mathrm{erg} / \mathrm{cm}^{3}$, exchange constant $A=3 \times 10^{-6} \mathrm{erg} / \mathrm{cm}$ [226], $\alpha=0.2$, and $\beta=0.4$. The grid size is $2 \mathrm{~nm}$.

\subsubsection{Densely Packed Magnetic Vortices}

Strip domains are very common in thin films with weak perpendicular anisotropy [225], and usually $K_{u} / K_{d}=Q<1$, where $K_{u}$ is uniaxial anisotropy constant with the easy axis perpendicular to the film and $K_{d}=0.5 \mu_{0} M_{s}$ is stray field energy density. When $K_{u}<K_{d}$, all the magnetizations prefer to lie in the plane for very thin film due to the large stray field energy, but for film thickness beyond one critical value $D_{c r}$, magnetizations will begin oscillating periodically out of the plane to save part of anisotropy energy. It is such periodical oscillation that results in a series of vortices which can be seen from the cross section of film. According to the rigorous theory of strip domain nucleation [225], for very small $Q$ (usually $Q<0.1$ ), the critical film thickness $D_{c r}$ and strip domain width $W_{c r}$ will be

$$
D_{c r}=2 \pi \sqrt{A / K_{u}}, \quad W_{c r}=D_{c r} .
$$

It indicates that such densely packed stripe domains can exist when film thickness is larger than a critical value $D_{c r}$, which is just twice the width of DW in the bulk, and the 
stipe domain width at critical film thickness is equal to the critical thickness, i.e., twice of DW width. For larger $Q, D_{c r}$ becomes smaller, and when $Q$ is large enough to approach the value of unity, the critical film thickness decreases to zero but the stripe domain width diverges.

For nanowire with weak perpendicular anisotropy, we propose that similar behaviors may also take place for the same reason, competition between stray field energy (although it is reduced in nanowire) and anisotropy energy. Therefore, densely packed magnetic vortices instead of stripe domains may be formed in the nanowire. Though it can't be exactly explained by the rigorous theory of stripe domain nucleation in thin film, some predictions can also be made for that in a nanowire. Using the anisotropy constant of Co, we can estimate the critical thickness $D_{c r}=2 \pi \sqrt{A / K_{u}} \approx 48 \mathrm{~nm}$ for thin film, with very small $Q$ assumed. Actually, the value of $Q \approx 0.4$ for Co is not so small, and for Co nanowire, this effective value can be somewhat large due to the reduced value of $K_{d}$ caused by the shape effect, and hence the exact critical thickness or nanowire width will be less than $48 \mathrm{~nm}$ for the Co nanowire, which has been confirmed by our simulations results, as shown in Fig. 4.5.1 (a) and (b) with square cross sections (a=b) of $48 \mathrm{~nm} \times 48 \mathrm{~nm}$ and $40 \mathrm{~nm} \times 40 \mathrm{~nm}$, respectively. All the domain structures shown in Fig. 4.5.1 are obtained by applying a saturated magnetic field pointing $+\mathrm{X}$-direction at first and then removing it, and after that magnetic vortices begin nucleated by thermal fluctuation which is effectively introduced by randomized magnetic field with proper amplitude in our simulations. It is noted that the shape of vortex core is rather ellipse than circular due to the anisotropy along Z-direction. Our simulations results indicate that for 
Co nanowire with square cross section, when the nanowire width is $40 \mathrm{~nm}$ or larger, densely packed vortices can be formed spontaneously after removing the field, but for the width less than 40nm, vortices can't be formed any more, which means the critical nanowire width for densely packed vortices nucleation is about $40 \mathrm{~nm}$ for the Co nanowire with square cross section. However, the exact critical vortex width can't be given from our simulations as easily as the critical nanowire width due to the periodical conditions, but it seems that $48 \mathrm{~nm}$ is more or less a reasonable value for vortex width for both Fig. 4.5.1 (a) and (b). Besides of square cross sections, Fig. 4.5 .1 (c) and (d) also show the cases of $a>b(48 \mathrm{~nm} \times 36 \mathrm{~nm})$ and $\mathrm{a}<\mathrm{b}(24 \mathrm{~nm} \times 48 \mathrm{~nm})$, respectively, and both cases show nucleated densely packed vortices, but with different vortex widths. For the case $\mathrm{a}>\mathrm{b}$, it more or less resembles that of thin film, and when a approaches infinity, densely packed stripe domains are restored. For the case of $a<b$, however, it resembles the Permalloy thin film or nanowire with IMA but with additional IMA component along Z-direction, and hence these vortex cores may perform similar distinct dynamic properties. It is noted that for the case of $a<b$, the vortex width is $72 \mathrm{~nm}$, as shown in Fig. 4.5.1 (d). All the above cases indicate that densely packed magnetic vortices can be formed in Co nanowires.

The existence of densely packed vortices also indicates that vortices won't annihilate each other, nor reverse the polarity of the neighbor vortex. All the vortices in Fig. 4.5.1 show positive core polarity $(\mathrm{p}=+1)$, while since racetrack memory requires two types of information carriers, vortices with negative core polarity $(\mathrm{p}=-1)$ are also considered, and the relative energy are also compared, which is shown in Fig. 4.5.2. 
According to Fig. 4.5.2, all the cases with densely packed vortices have lower energy than single domain, which further explains the existence of densely packed vortices

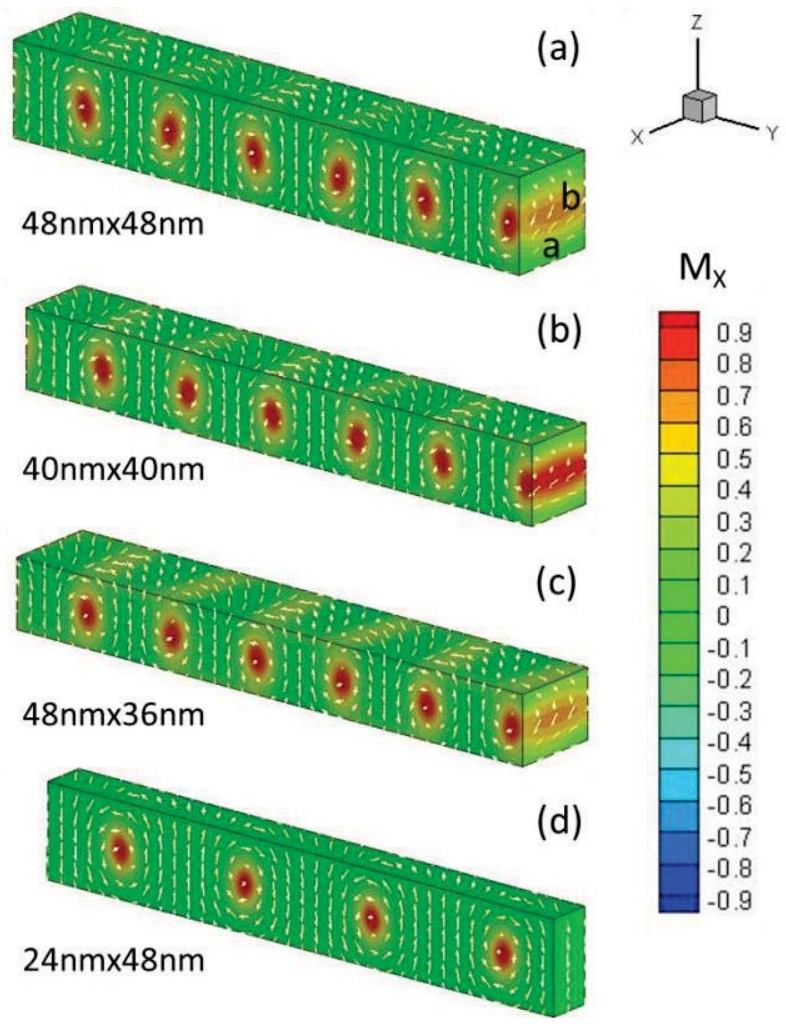

Figure 4.5.1 Domain structures of densely packed vortices formed in nanowires with cross sections (a $\times$ b) of (a) $48 \mathrm{~nm} \times 48 \mathrm{~nm}$, (b) $40 \mathrm{~nm} \times 40 \mathrm{~nm}$, (c) $48 \mathrm{~nm} \times 36 \mathrm{~nm}$, and (d) $24 \mathrm{~nm} \times 48 \mathrm{~nm}$. The easy axis is along Z-direction and Nanowire with length of $48 \mathrm{~nm} \times 6=288 \mathrm{~nm}$ is periodically repeated along Y-direction. White arrows indicate magnetizations and the color indicates the value of $\mathrm{M}_{\mathrm{x}}$. All the vortices are obtained by applying a saturated magnetic field pointing $+\mathrm{X}$ direction at first and then removing it.

discussed above. Although the single domain has larger energy, it can't be treated as unstable phase, and actually, it is very difficult to be destroyed by the thermal fluctuation and behaves somewhat large stability according to our simulations study. After all, our densely packed vortices are not made from such kind of single domain state. Energy of nanowires with six vortices consisting of $0,1,2$ and 3 vortices with $p=-1$ are plot as four 
points almost in the same level as shown in Fig. 4.5.2. Pairs of vortices cores with positive and negative polarity can lower the energy by reducing the stray field energy, and then vortices with uniform core polarity have the largest energy, vortices with mixed core polarity have lower energy, and the ones with arranged $\mathrm{p}=+1$ and $\mathrm{p}=-1$ core pairs will have the lowest energy. The energy difference between these vortices cases is within $0.1 \mathrm{eV}$, very small energy if portioned to a single vortex, and such a small value is just in the same order with thermal fluctuation which is too small to reverse core polarity, indicating the good stability of core polarity that can be used as information bit to realize magnetic nonvolatile random access memory and VRM.

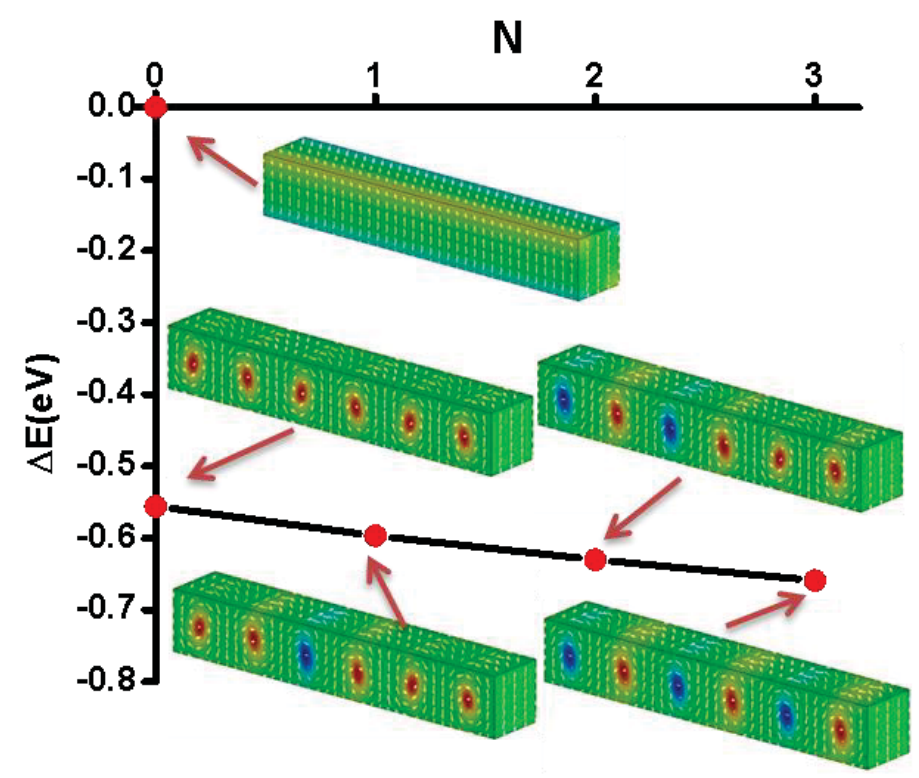

Figure 4.5.2 Domain structures as well as their total energy for one nanowire of single domain with magnetizations along easy axis and four nanowires of six densely packed vortices with $\mathrm{N}=0$, 1,2 and 3 cores of negative polarity ( $\mathrm{p}=-1$, indicated by blue color). The size of these nanowires are $48 \mathrm{~nm} \times 48 \mathrm{~nm} \times 288 \mathrm{~nm}$. The energy for single domain with magnetization along the easy axis is treated as reference with its energy set to zero.

\subsubsection{Magnetic Vortex Racetrack Memory}


The good stability of both the densely packed vortices and their core polarity makes themselves good candidate as information carriers of magnetic nonvolatile random access memory. If current induced magnetic vortex motion is taken into account [57, 222], a novel racetrack memory can be proposed based on densely packed vortices instead of traditional DWRM, and such magnetic VRM will have unique properties different from DWRM.

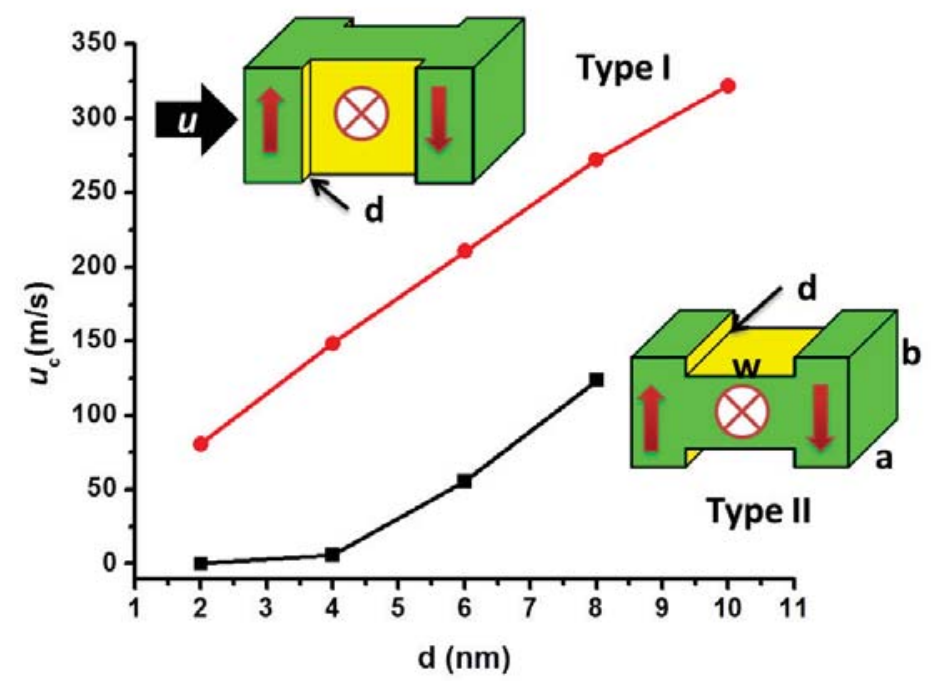

Figure 4.5.3 Critical current density $u_{c}$ for a single vortex depinned from the notch of $48 \mathrm{~nm} \times 48 \mathrm{~nm} \times \mathrm{d}$ in the nanowire with cross section $48 \mathrm{~nm} \times 48 \mathrm{~nm}$, where $\mathrm{d}$ (about 2 10nm) is depth of the notch. For type I, the notch crosses the vortex core, while for type II, the notch crosses from the outside of the core.

Pinning sites are always required for positioning and transportation of magnetic carriers driven by spin-polarized current in racetrack memory process, so we start with the determination of the critical current density for a vortex depinned from two types of pinning notches, as shown in Fig. 4.5.3. For type I, the notch crosses vortex core, while for type II, the notch crosses from the outside of the core. Various values of $\mathrm{d}$ are 
considered to determine the critical depinning current density $u_{c}$. It is noted from Fig. 4.5.3 that for the same d, Type I has stronger pinning effect and higher $u_{c}$ than Type II. The reason for that is obvious. The energy for a vortex is concentrated within the core due to the fast varying spin texture, while the outer part contributes less to the energy. Therefore, for Type I notch, the energy barrier to be overcome for depinning is higher than Type II. In addition, the critical depinning current density $u_{c}$ seems to change linearly with the notch depth $\mathrm{d}$ for Type I while shows an exponent-like relationship for Type II, both of which are for the same reason. Since Type I notch can effectively pin the vortex, we will use that type of notch for the design of our VRM.

Unlike DWRM, for which each bit or domain magnetization should be confined and distinguished by notches that are used to pin DWs, VRM does not require such a confinement for each vortex since it is vortices instead of domain magnetizations that carry the bits and those vortices can be packed densely without annihilated. Of course, one can also confine each vortex by a notch for VRM design, but such design will not show its unique property different from DWRM, and more pinning sites will need larger current density. Although it is not necessary to confine every vortex, at least one notch is needed to confine the vortex that is chosen to read and write its bit information shown by the core polarity. Therefore, two designs are proposed for VRM, one with only one notch positioned at the nanotrack center, and the other with a half number of notches used in DWRM positioned in the right half-track, which are shown in Fig. 4.5.4 (a) and Fig. 4.5.5 (a), respectively. All the notches are Type I notches with depth of 4nm. Five bits '11001' are artificially written in our simulation, and these bits are stored in the left half-track while no any bits stored in the right half-track. The bit confined by the notch centered in 
the track is the one to be read and written, but the implement of bit reading and writing for vortex core is not considered in this paper, and only the concept of VRM and its unique transportation properties under spin-polarized current are focused. The general process for the VRM is: only the vortex trapped in the central notch can be read and written, so any target vortex prepared to read or write should be moved into the central notch at first; each target vortex can be driven to the central notch at one time by applying a proper current; remove the applied current to read or write for the target vortex; after finishing reading or writing, a large current with opposite direction (for Design I, $\mathrm{u}=-87 \mathrm{~m} / \mathrm{s}$, while for Design II, $\mathrm{u}=-130 \mathrm{~m} / \mathrm{s}$ ) is applied in order to restore the initial state.

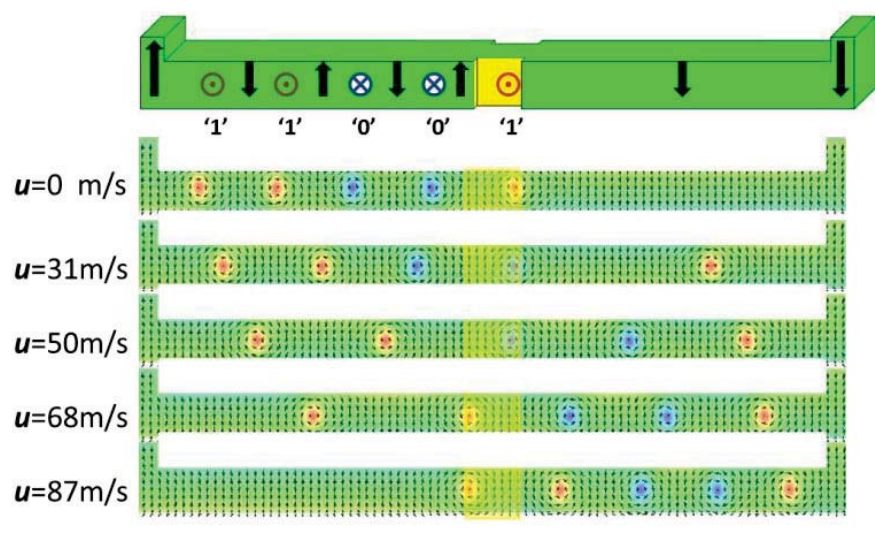

(a)

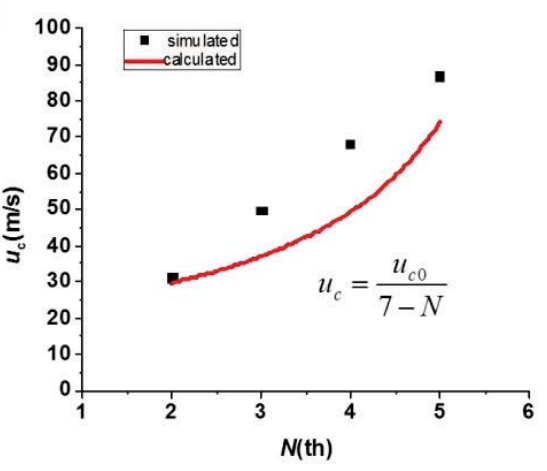

(b)

Figure 5.4.4 (a) Design I for the VRM in a nanowire with total length of 720nm and square cross section $48 \mathrm{~nm} \times 48 \mathrm{~nm}$ for the main part while $48 \mathrm{~nm} \times 72 \mathrm{~nm}$ for the edge. The only notch is $48 \mathrm{~nm} \times 48 \mathrm{~nm} \times 4 \mathrm{~nm}$, located at the center of the nanotrack. For the initial state, five bits ' 11001 ' are in the left half-track while no any bits stored in the right half-track. All the domain structures are obtained by applying a current of $u=0,31,50,68$ or $87 \mathrm{~m} / \mathrm{s}$ at first to shift the first, second, third, fourth or fifth vortex to the central notch and then removing the current to make the domain structures fully relaxed. (b) Simulated (black squares) and calculated (red line) critical currents required to move the $N$ th vortex in or move the $(N+1)$ th vortex out of the notch. $u_{c}=148.5 \mathrm{~m} / \mathrm{s}$. 
For Design I, there is only one notch which is located at the center of the whole nanotrack. For the initial state or the state without current applied, five densely packed vortices are stored in the left half-track with one of them trapped within the central notch, but for the right half-track, there is only single domain state without any vortices stored in, and the domain structure is shown by the case of $\mathrm{u}=0$ in Fig. 4.5.4 (a). The stray field generated by the single domain at the right half-track will push the first vortex to move to the right, and since it is easier for a vortex to move into a notch than to move out of it, the first vortex will be moved into and trapped by the notch, i.e., the reading and writing for the first vortex doesn't need current applied. In order to read or write for the second vortex, the first one must be moved out of the notch to allow the second one move in. The critical current to move the first vortex out or move the second one in is $u=31 \mathrm{~m} / \mathrm{s}$, which is much smaller than the critical depinning current $(\sim 150 \mathrm{~m} / \mathrm{s})$ for a single vortex trapped in the notch. The reduced depinning critical current is due to the vortex pile-up effect discussed in Sec. 4.3, and each of the packed vortices contributes a driving force caused by spin transfer torque effect, i.e., in additional to its own driving force which is caused directly by the current, all the other vortices behind it can also push it with each one supplying the same driving force, and hence the critical current for vortex depinning is reduced. The more the vortices behind, the smaller the critical depinning current required. Both the simulated and calculated critical currents required to move the $N$ th vortex in or move the $(N+1)$ th vortex out of the notch are plot in Fig. 4.5.4 (b). Since the calculated results are based on analysis for the ideal pile-up effect without taking notches and stray field into account, there are some deviations between these two set of results. Actually, the existence of a notch usually prevents the behind vortex approaching to the one 
trapped in the notch due to the relatively small energy barrier caused by the left edge of the notch, which means higher current is required to overcome the additional energy barrier. The stray field effect seems somewhat complex. Briefly speaking, the stray field tends to make the vortices distributed evenly, as shown in Fig. 4.5.4 (a). Therefore, for the cases of $\mathrm{u}=0,31$ and $50 \mathrm{~m} / \mathrm{s}$, the stray field at the right prefers to depin the trapped vortex, while for the cases of $\mathrm{u}=68$ and $87 \mathrm{~m} / \mathrm{s}$, the stray field at the left prefers to draw the trapped vortex back. But for the current induced vortex motion existed in all cases, the piled vortices before the notch will become denser, and the stray field prefers to evenly distribute those vortices, which reduces the pile-up effect and makes the critical depinning current higher. Although it is difficult to quantitatively define these effects, it is known that the total effect makes the critical current higher than the calculated ideal case according to Fig. 4.5.4 (b).

For Design II, five notches are introduced in the right half-track to trap depinned vortices. All the relaxed domain structures after applying critical current $u=0,50,74,105$ and $130 \mathrm{~m} / \mathrm{s}$ are shown in Fig. 4.5.5 (a). Both the simulated and calculated critical currents required to move the $N$ th vortex in or move the $(N+1)$ th vortex out of the notch are plot in Fig. 4.5.5 (b). The calculated results are obtained considering the ideal vortex pile-up effect, and hence manifesting a linear relationship unlike that for Design I. It is noted that the simulated value of $u_{c}$ is larger than the calculated one, and just like Design I, such a deviation is caused by the effects of notches and stray field. Comparing the two designs, we found that the critical depinning current $u_{c}$ for Design II is larger than Design I due to its more notches, but a linear relationship is predicted based on the ideal pile-up 
assumption unlike that of predicted for Design I. For the simulation results, however, both cases show not bad linearity by including those complex effects.

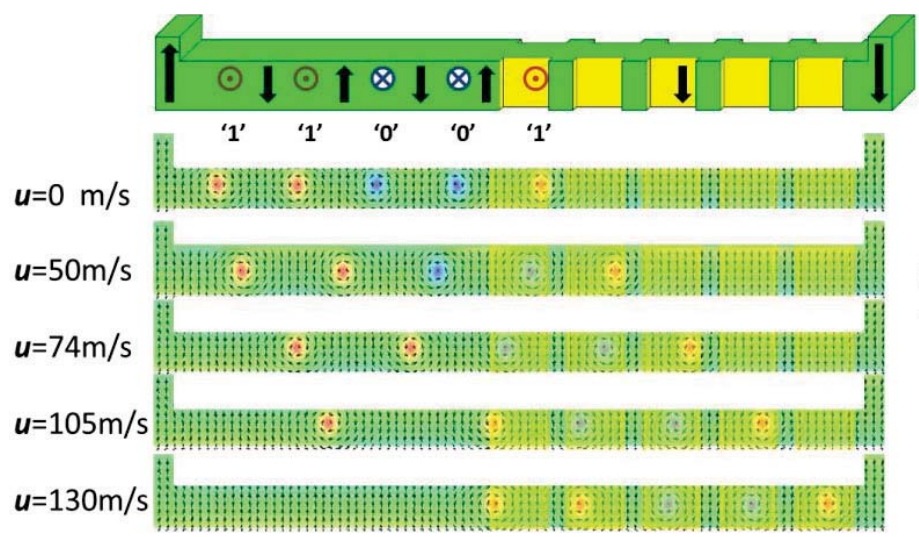

(a)

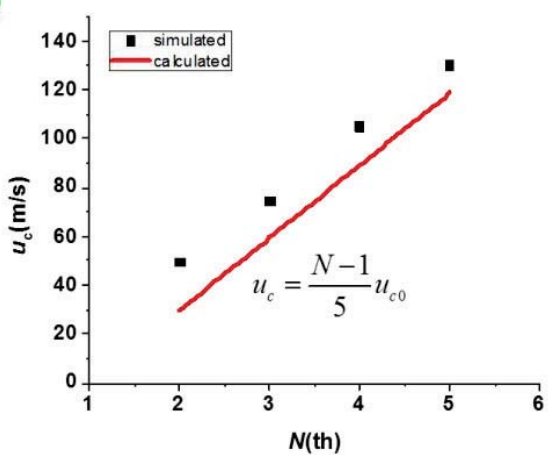

(b)

Figure 4.5.5 (a) Design II for the VRM in a nanowire with total length of $720 \mathrm{~nm}$ and square cross section $48 \mathrm{~nm} \times 48 \mathrm{~nm}$ for the main part while $48 \mathrm{~nm} \times 72 \mathrm{~nm}$ for the edge. All the five notches are $48 \mathrm{~nm} \times 48 \mathrm{~nm} \times 4 \mathrm{~nm}$, located at the right half of the nanotrack. For the initial state, five bits '11001' are in the left half-track while no any bits stored in the right half-track. All the domain structures are obtained by applying a current of $\mathrm{u}=0,50,74,105$ or $130 \mathrm{~m} / \mathrm{s}$ at first to shift the first, second, third, fourth or fifth vortex to the central notch and then removing the current to make the domain structures fully relaxed. (b) Simulated (black squares) and calculated (red line) critical currents required to move the $N$ th vortex in or move the $(N+1)$ th vortex out of the central notch. $u_{c}=148.5 \mathrm{~m} / \mathrm{s}$.

Based on these two types of designs for VRM, some unique properties different from DWRM can be demonstrated: 1) the bit carriers for VRM are vortices with size twice of the DW width while for DWRM are domains with size much larger than the DW width, which indicates potential higher density storage for VRM; 2) it is not required to confine every bit carrier using a notch for VRM, because, unlike DWs, densely packed vortices won't annihilate each other and those individual vortex cores don't need to be 
distinguished by notches; 3) for DWRM, multiple current pulses should be applied to shift the target DW to the specified location, while for VRM, only one constant current is required to shift the target vortex to such location because of the vortex pile-up effect; 4) for DWRM, the critical depinning current can be reduced by using nanowire with PMA, while for VRM, the critical depinning current is reduced due to the pile-up effect and can be further reduced by adding some null vortices to each side of nanotrack.

\subsubsection{Conclusion}

Racetrack memory which is based on current induced DW motion was proposed to have ultrahigh density storage, while magnetic vortex also reveals attractive static and dynamic properties manifested by its core polarity, and their combination, VRM, will be proposed to possess unique properties which must be different from traditional DWRM. However, most of the researches are focused on magnetic vortices in Permalloy nanodisk or nanowires which are improper to be employed for the implement of VRM. Therefore, densely packed vortices in Co nanowire is proposed based on the notion of densely packed stripe domains which can be spontaneously formed in a thin film with weak perpendicular anisotropy. Our simulation results have confirmed the existence of such densely packed vortices in Co nanowire as well as the good stability of these vortices and their core polarity. These densely packed vortices may also be potential candidate for magnetic nonvolatile random access memory except the VRM. Two designs of VRM which are based on densely packed vortices and current induced vortex motion are demonstrated to study current induced vortex motion behaviors in the nanowire. According to our simulations results, the novel VRM shows unique properties 
different from DWRM, such as the small vortex width (only twice of DW width), the less notches requirement, the one-time constant current transportation, and the reduced depinning critical current due to the pile-up effect. Such unique properties of VRM will have potential applications in the field of spintronics. 


\subsection{Future Research}

1. Researches on domain wall structures as well as the current induced properties in nanowire systems including DMI effect will be carried out by employing the phase filed model and micromagnetic simulations in the future, and the related novel functionalities based on these spin-orbit torques will also be explored.

2. Since the magnetocrystalline anisotropy of a ferromagnetic system can be modulated by the electric field, the domain wall structure and properties which strongly depend on the anisotropy might be affected indirectly by the electric field. Since the quantitative respond of the anisotropy constant with respect to external electric field can be calculated by ab initio method, the electric field modulated structures, properties and functionalities of domain walls can be studied by us through combining both ab initio calculations and micromagnetic simulations.

3. Rather than magnetic field and spin-polarized current, stress or strain field can also provide a driving force for domain walls in nanowires, and therefore, stress/strain induced domain wall motion will be also studied by us in the future.

4. Spin waves propagation can also drive domain walls to move in some manner, and then we will use micromagnetic simulations to investigate the spin-wave induced domain wall motion under DMI effect. 


\section{Chapter 5. Magnetic Domain Walls in Atomic Chains}

\subsection{Introduction}

Low-dimensional magnetic systems such as ultrathin films, nanowires and nanostructures have attracted much interest in recent years because of their unique properties which conventional two-dimensional (2D) and 3D magnetic systems don't occupy. In particular, intensive investigations have been done on 1D atomic chain magnetic systems which play an important role in nanomagnetism and nanospintronics for the attractive potential applications in high-density recording devices, spin-based logic elements, and spin-transport devices. [80, 106, 227-231] All of those will ultimately relate to the magnetic domain and domain wall in the atomic chain. For example, the ballistic magnetoresistance is strongly dependent of domain wall size and also affected by the magnetic moment softening within domain wall [93, 104]; data recording can be realized by single domain finite atomic chain deposited on surface and its stability depends on the energy barrier which relates to the magnetocrystalline anisotropy energy (MAE) [227, 228]. MAE is an utmost important factor in 1D atomic chain system, without which an infinite atomic chain even can't maintain ferromagnetism according to the isotropic Heisenberg model [232]. What's more, MAE directly affects the domain wall width and energy barrier. Higher MAE usually leads to narrower domain wall and higher energy barrier, and hence able to enhance the corresponding ballistic magnetoresistance and domain stability to improve device performance while reducing its size. Thus, it is of fundamental importance to study the atomic domain wall structure and domain stability of a truly $1 \mathrm{D}$ magnetic atomic chain with relatively higher MAE. 
A great deal of theoretical calculations at ab initio DFT level for $3 \mathrm{~d}, 4 \mathrm{~d}$ and $5 \mathrm{~d}$ transition-metal atomic chains, either freestanding [103, 112, 233-240] or supported [233, 241-246], have been reported. Although usually only 4d and 5d transition-metal monoatomic chains are thought to have large MAE, some $3 \mathrm{~d}$ transition-metal like Ni freestanding monoatomic chain for instance has been reported to also have giant MAE ( $12 \mathrm{meV} /$ atom [112]) which is comparable with those of $4 \mathrm{~d}$ transition-metal chains (6 12meV/atom [237]). What's more, the ferromagnetic state of monoatomic chain of $\mathrm{Ni}$, as well as other $3 \mathrm{~d}$ transition-metals such as Fe, Co and V, have been reported almost half-metalic[112], a fascinating perspective for spin-dependent transport device, and suspended Co monoatomic chain with a fully polarized conductance channel has been experimentally observed [247]. Large magnetoresistance can be expected for the nearly half-metalic Ni atomic chain when the domain wall is narrow enough, and such a ballistic magnetoresistance has been theoretically calculated for atomic-size Ni nanowires with width-fixed domain wall of spin-spiral structure $[103,104]$ and experimentally observed for nano/atomic contacts of $\mathrm{Ni}$ nanowires [93, 96]. Magnetic moment softening in artificially confined narrow domain wall of $\mathrm{Ni}$ atomic nanowire, which is due to the hybridization between noncollinear spin states, was also reported to be able to enhance the ballistic magnetoresistance [104]. However, the domain wall for a truly magnetic monoatomic chain has deterministic wall width and its structure will not be as simple as spin-spiral since the MAE and shape anisotropy will apply. For those reasons, the freestanding Ni monoatomic chain is focused in this paper and the truly domain wall structure will be investigated. 
For the itinerant ferromagnets like $\mathrm{Ni}$, the hybridization effect between two spin channels usually exists, while for the ferromagnets with well localized d electrons like Fe or most of $4 \mathrm{~d}$ transition-metals, that effect is weak. Although the spin moment softening effect is universally existed in itinerant ferromagnetic materials, it is rarely investigated by most $a b$ initio studies. It can be expected that some magnetic parameters, such as exchange interaction parameters and MAE constants, might be affected by the spin moment softening effect. Although such softening is ignorable for slowly varying spin textures or wide domain walls, for the magnetic system with larger MAE, like Ni atomic chain, the domain wall will be very narrow and the spin moment softening effect might be relatively evident. Especially for the spin dynamics process with intense thermal fluctuations, the softening effect might exist everywhere, which may affect spin dynamics behaviors in a certain manner. Therefore, for the truly domain wall structure of the freestanding Ni monoatomic chain, both MAE and spin moment softening effects should be considered. Since the ab initio method alone is powerless to obtain accurate domain wall structure but able to obtain corresponding magnetic parameters, we will employ the atomistic model by using parametrized Hamiltonian terms.

Theoretical details for the $a b$ initio adiabatic spin dynamics in magnets can be found in Ref. [248], in which the evolution is described by the time dependent orientational configurations determined by the equation of motion considering thermal effects with the form coinciding with the stochastic Landau-Lifshitz-Gilbert (LLG) equation [153]. The atomistic model based on stochastic LLG equation can successfully describe the spin dynamics under thermal fluctuations and/or external magnetic field, and lots of atomistic spin dynamics simulations have been done for atomistic magnetic 
systems [249-253]. However, most atomistic spin dynamics simulations are applied to systems in which the spin moment magnitude is conserved and the orbital moment is very small compared with the spin moment. For the 1D Ni atomic chain system in which both the spin moment softening effect and the orbital moment should be considered, this paper shows that such atomistic model is still valid and both the spin and orbital moments can be obtained through the spin orientation configurations. Thus, the domain wall structure and the spin dynamics under thermal effect can be understood through the atomistic model. Since for magnetic recording, the ferromagnetic single domain stability against thermal fluctuations is quite essential, and high MAE can increase the stability while shortening the required chain length, this paper also focuses on the ferromagnetic single domain stability and switching process of 1D freestanding Ni monoatomic chain with finite length at finite temperature by employing that atomistic model. Although some magnetic properties for $\mathrm{Ni}$ atomic chains which are deposited on substrates [241, 242, 245] or encapsulated inside nanotubes [107] might be different, the underlying spin dynamics behaviors would share the same mechanism, so that the present work can also shed a light on other magnetic atomic chain systems with high MAE.

We organize this paper as follows. In Sec. II, we use ab initio technique to calculate relevant magnetic parameters for the freestanding Ni monoatomic chain at zero temperature, and the parameters at finite temperature are also calculated based on a DFT approach to the electronic free energy. In the front of those calculations, the computational details are presented first. In Sec. III, an atomistic magnetic model for the $\mathrm{Ni}$ monoatomic chain is introduced and spin dynamics simulations based on that model are performed to study the magnetic domain wall structure as well as the ferromagnetic 
single domain stability and switching behaviors under thermal fluctuations. In Sec. IV, the main conclusions in this paper are summarized.

\subsection{Ab initio Studies}

\subsubsection{Computational Details}

First-principles calculations within the framework of DFT are done with the Vienna ab initio simulation package (VASP 5.2) [124, 254-256]. For such calculations, the plane-wave basis projector augmented wave (PAW) method $[257,258]$ was used in the framework of the spin polarized generalized gradient approximation (GGA) in the Perdew-Burke-Ernzerhof form [259, 260]. The plane-wave cutoff energy of $500 \mathrm{eV}$ is used, and the total energy convergence criterion is $10^{-5} \mathrm{eV}$. The distance for the neighbor $\mathrm{Ni}$ atomic chains is as large as $20 \AA$ to avoid the interactions between each other. The Monkhorst-Pack $k$-point scheme of $1 \times 1 \times 101$ is used.

Noncollinear magnetism calculations [125] in the PAW formalism are performed for the relevant parameters of $\mathrm{Ni}$ atomic chain. For the calculations of exchange interaction parameters as well as the relevant spin moment softening coefficients, the spin-spiral states with propagation vector $\mathbf{q}$ in the frozen-magnon approach are considered. For the calculations of MAE constants and orbital moments with respect to the angle $\theta$ between the spin orientation and easy axis, the spin-orbit coupling (SOC) is included and a series of $\theta$ are considered. Because of the spin moment softening effect, MAE values with constrained spin moments are also calculated. 


\subsubsection{Magnetic Parameters at Zero Temperature}

Based on our ab initio calculations, the structurally optimized atomic bond length for the equilibrium ferromagnetic $\mathrm{Ni}$ chain is $d=2.18 \AA$. Noncollinear magnetic calculations of $\mathrm{Ni}$ monoatomic chain at several different bond lengths around the equilibrium value are also taken into account. Spin-spiral magnetic structures of propagation vector $\mathbf{q}$ along [001] direction with different $\mathbf{q}$ values up to $0.3(2 \pi / d)$ are applied to calculate the corresponding spin moments and total energies, as shown in Fig. 5.1 and Fig. 5.2, respectively. The case of $\mathrm{q}=0$ corresponds to the collinear ferromagnetic state and $\mathrm{q}=0.25$ corresponds to the state for which the angle between neighbor spins is $90^{\circ}$. According to Fig. 5.1 (a), it is clear that the spin moment is reduced with increased spiral angles due to the hybridization between noncollinear spin states. The larger the angle, the stronger the hybridization effect, the smaller the spin moment. It is noted from Fig. 5.1 (a) that the spin moment is only slightly reduced for small q while evidently reduced for somewhat larger q. For example, it is only $\sim 5 \%$ reduction when $\mathrm{q}=0.1$ (or spiral angle is $36^{\circ}$ ), but $33 \%$ reduction for $\mathrm{q}=0.25$ (or spiral angle is $90^{\circ}$ ). Such a spin moment softening effect was also reported for other itinerant magnetic systems with spinspiral states. [248] In order to study the q dependent of spin moment softening effect, we assume that the atomic spin moment is affected only by the spin orientations of near atoms according to

$$
M_{S}=M_{0}+\sum_{i=1}^{n} a_{i}\left(\mathbf{m}_{-i} \cdot \mathbf{m}_{0}+\mathbf{m}_{0} \cdot \mathbf{m}_{i}\right),
$$


where $M_{S}$ is the atomic spin moment, $M_{0}$ and $a_{i}$ are the spin moment softening coefficients, $\mathbf{m}_{0}$ and $\mathbf{m}_{ \pm i}$ are the spin orientations of the atom and its $i$ th nearest atoms, respectively. For the spin-spiral state, Eq. (5.1) reads

$$
M_{S}=M_{0}+\sum_{i=1}^{n} 2 a_{i} \cos (2 \pi i q)
$$

Fig. 5.1 (a) shows the well fitted curve for the $a b$ initio calculated spin moments according to Eq. (5.2) up to $n=3$, and the fitting coefficients are shown in Fig. 5.1 (b). Atom chains with bond lengths of $d=2.15 \sim 2.20 \AA$ are considered, and all the corresponding fitting coefficients are shown in Fig. 5.1 (b). It is noted that those coefficients don't change much with the variation of bond length $d$, and both $M_{0}$ and $a_{1}$ contribute most to the spin moment softening effect while higher order coefficients $a_{2}$ and $a_{3}$ play a minor effect due to their small magnitude. Based on those four coefficients, nearly zero spin moment can be expected for $\mathrm{q}=0.5$, the antiferromagnetic state, which will manifest the most significant hybridization effect.

The total energies for the spin-spiral state with different $\mathrm{q}$ values of $\mathrm{Ni}$ monoatomic chain with $d=2.18 \AA$ are shown in Fig. 5.2 (a), which is in good agreement with Ref. [235]. Larger q results in higher energy, indicating ferromagnetic state is the stable state for $\mathrm{Ni}$ atomic chain. The full expansion for the effective Heisenberg Hamiltonian is given by [261] 


$$
\begin{aligned}
H_{e x c h}= & -\frac{1}{2} \sum_{i j} J_{i j} \mathbf{M}_{S i} \cdot \mathbf{M}_{S j}-\frac{1}{2} \sum_{i j} J_{i j}^{(2)}\left(\mathbf{M}_{S i} \cdot \mathbf{M}_{S j}\right)^{2}-\ldots-\frac{1}{2} \sum_{i j} J_{i j}^{(n)}\left(\mathbf{M}_{S i} \cdot \mathbf{M}_{S j}\right)^{n} \\
& -\frac{1}{4 !} \sum_{i j k l} J_{i j k l}\left[\left(\mathbf{M}_{S i} \cdot \mathbf{M}_{S j}\right)\left(\mathbf{M}_{S k} \cdot \mathbf{M}_{S l}\right)+\left(\mathbf{M}_{S j} \cdot \mathbf{M}_{S k}\right)\left(\mathbf{M}_{S l} \cdot \mathbf{M}_{S i}\right)\right. \\
& \left.+\left(\mathbf{M}_{S l} \cdot \mathbf{M}_{S i}\right) \times\left(\mathbf{M}_{S j} \cdot \mathbf{M}_{S k}\right)\right]-\ldots
\end{aligned}
$$

where $J_{i j}$ is the bilinear exchange interaction parameter, $J_{i j}^{(2)}$ the biquadratic exchange interaction parameter, $J_{i j k l}$ the four site exchange interaction parameter, and $J_{i j}^{(n)}$ the higher order exchange interaction parameter. Although the bilinear exchange term is important, the biquadratic exchange term is also though as important as the bilinear term $[262,263]$. It has been reported for fcc Ni that both the bilinear and biquadratic exchange terms for first nearest atoms are dominant [264], and we assume that the case for $\mathrm{Ni}$ atomic chain is also similar. Therefore, based on our assumption, only the first two terms of Eq. (5.3) for just the first nearest atoms are considered, i.e.,

$$
H_{e x c h}=-\frac{1}{2} \sum_{|i-j|=1} J \mathbf{M}_{S i} \cdot \mathbf{M}_{S j}-\frac{1}{2} \sum_{|i-j|=1} J^{(2)}\left(\mathbf{M}_{S i} \cdot \mathbf{M}_{S j}\right)^{2} .
$$

According to Eq. (5.4), the q-dependent total energy for the spin-spiral state will be

$$
E(q)=E(0)+J\left[M_{S}^{2}(0)-\cos (2 \pi q) M_{S}^{2}(q)\right]+J^{(2)}\left[M_{S}^{4}(0)-\cos ^{2}(2 \pi q) M_{S}^{4}(q)\right]
$$

where $M_{S}(q)$ is the q-dependent spin moment obtained from Eq. (5.2). Fig. 5.2 (a) shows that our ab initio calculated total energy values are fitted very well according to Eq. (5.5) and both the bilinear and biquadratic exchange interaction parameters for $d=$ 2.18 $\AA$ are shown in Fig. 5.2 (b). According to the fitting results, the magnitude for the biquadratic exchange parameter is about $1 / 4$ of that for the bilinear one but with an opposite sign, indicating that the biquadratic term can't be ignored, consistent with our 
above assumption. Exchange parameters for $\mathrm{Ni}$ atomic chain with different bond lengths are also shown in Fig. 5.2 (b). Interestingly, both $J$ and $J^{(2)}$ decrease almost linearly by increasing the bond length, which indicates weakened exchange interactions for lengthened bonds, and such a linearship has been reported due to the magnetoelastic effect [265].
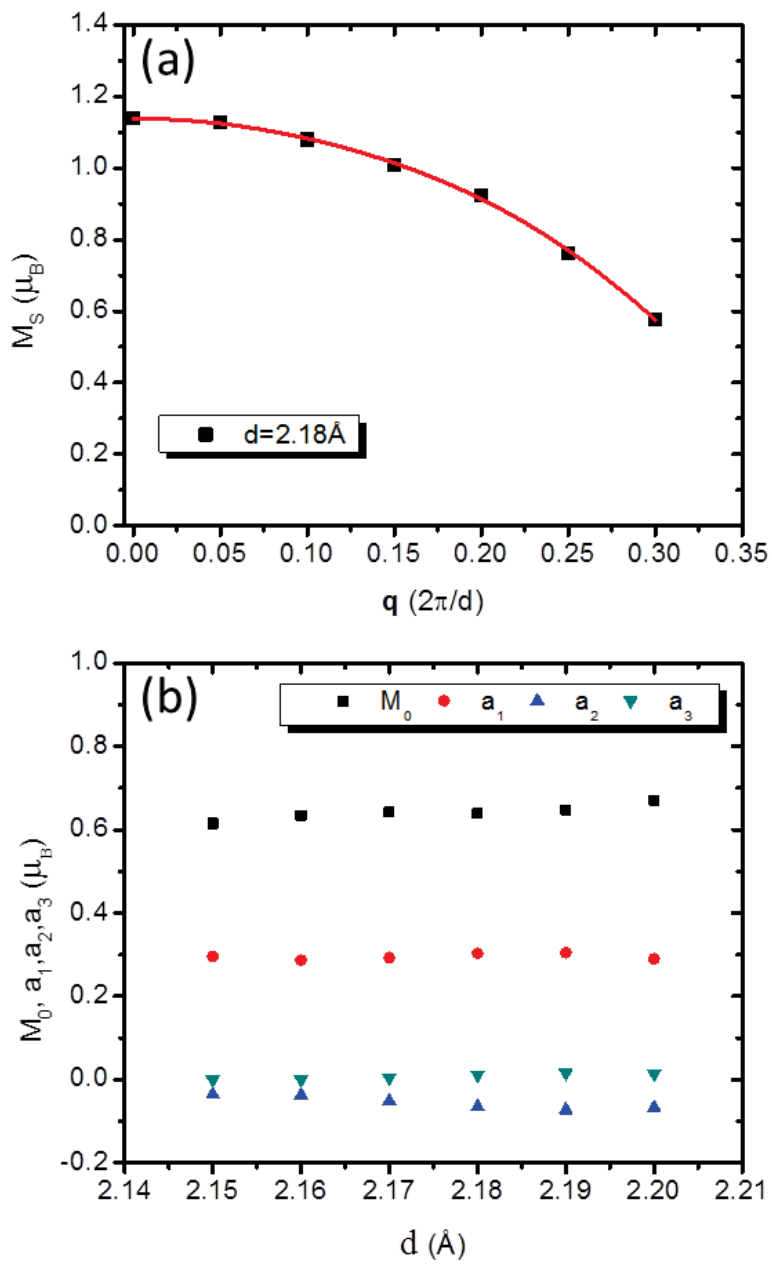

Figure 5.1 (a) Spin moments for spin-spiral state with different propagation q values for bond length $d=2.18 \AA$ A. Red curve represents the fitting for those spin moments according to Eq. (5.2) up to $n=3$. (b) The relevant fitting coefficients, $M_{0}, a_{1}, a_{2}$ and $a_{3}$, for $\mathrm{Ni}$ atomic chain with different bond lengths from $d=2.15 \AA$ to $2.20 \AA$. 

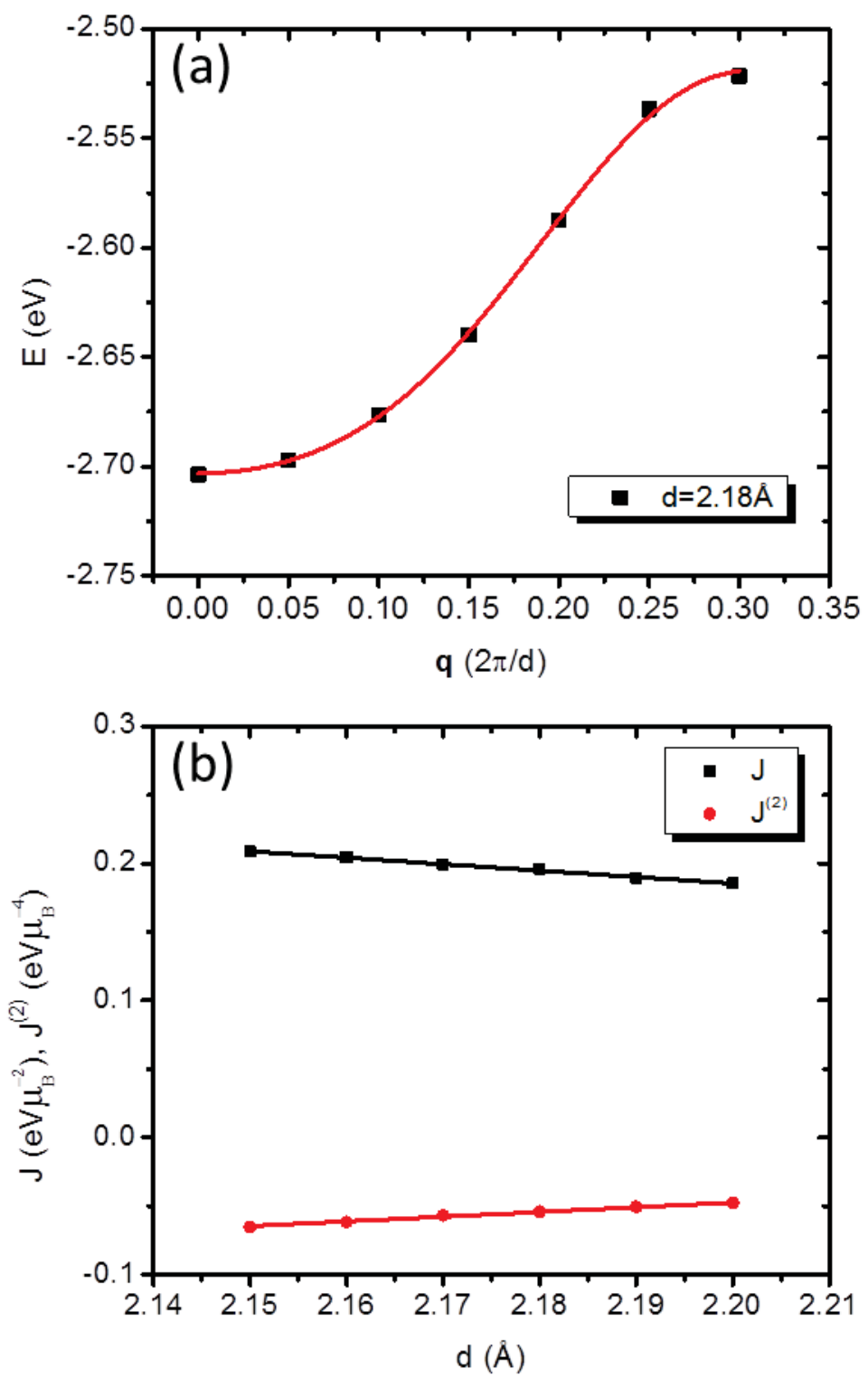

Figure 5.2 (a) Total energies for spin-spiral state with different propagation q values for bond length $d=2.18 \AA$. Red curve represents the fitting for those energies according to Eq. (5.5). (b) The bilinear and biquadratic exchange interaction parameters, $J$ and $J^{(2)}$, for Ni atomic chain with different bond lengths from $d=2.15 \AA$ to $2.20 \AA$. Both sets of parameters are linearly fitted vs. $d$. 
In order to calculate the MAE as well as the orbital moment, SOC is included in our ab initio calculations. For the Ni atomic chain, MAE is the energy difference between two magnetic moment directions, [100] and [001], where [001] is the chain direction and also the easy axis in our case. Based on our calculated energies shown in Fig. 5.3 (a), MAE in a form expended to the second order has been considered, i.e.,

$$
E=E_{0}+K_{1} \sin ^{2} \theta+K_{2} \sin ^{4} \theta
$$

where $K_{1}$ and $K_{2}$ are the first and second order MAE constants, and $\theta$ is the angle between the spin moment direction and easy axis. Curve fitting according to Eq. (5.6) has been made as shown in Fig. 5.3 (a) with the fitted values of $K_{1}$ and $K_{2}$ for the equilibrium bond length as well as the other bond lengths shown in Fig. 5.3 (b). For the equilibrium case, $K_{1}=10.8 \mathrm{meV}$ and $K_{2}=3.2 \mathrm{meV}$, and then the total MAE can be described as $K_{1}+K_{2}=14 \mathrm{meV}$, very close to the value obtained in Ref. [112], and such a large MAE usually lead to a very narrow domain wall. Similar with the above exchange interaction parameters, a linear trend is also observed for the MAE constants with a variation of bond length. By increasing bond length, $K_{1}$ decreases but $K_{2}$ increases, which indicates that the high order effect becomes more evident for elongated bond, although $K_{1}$ is still dominant. 

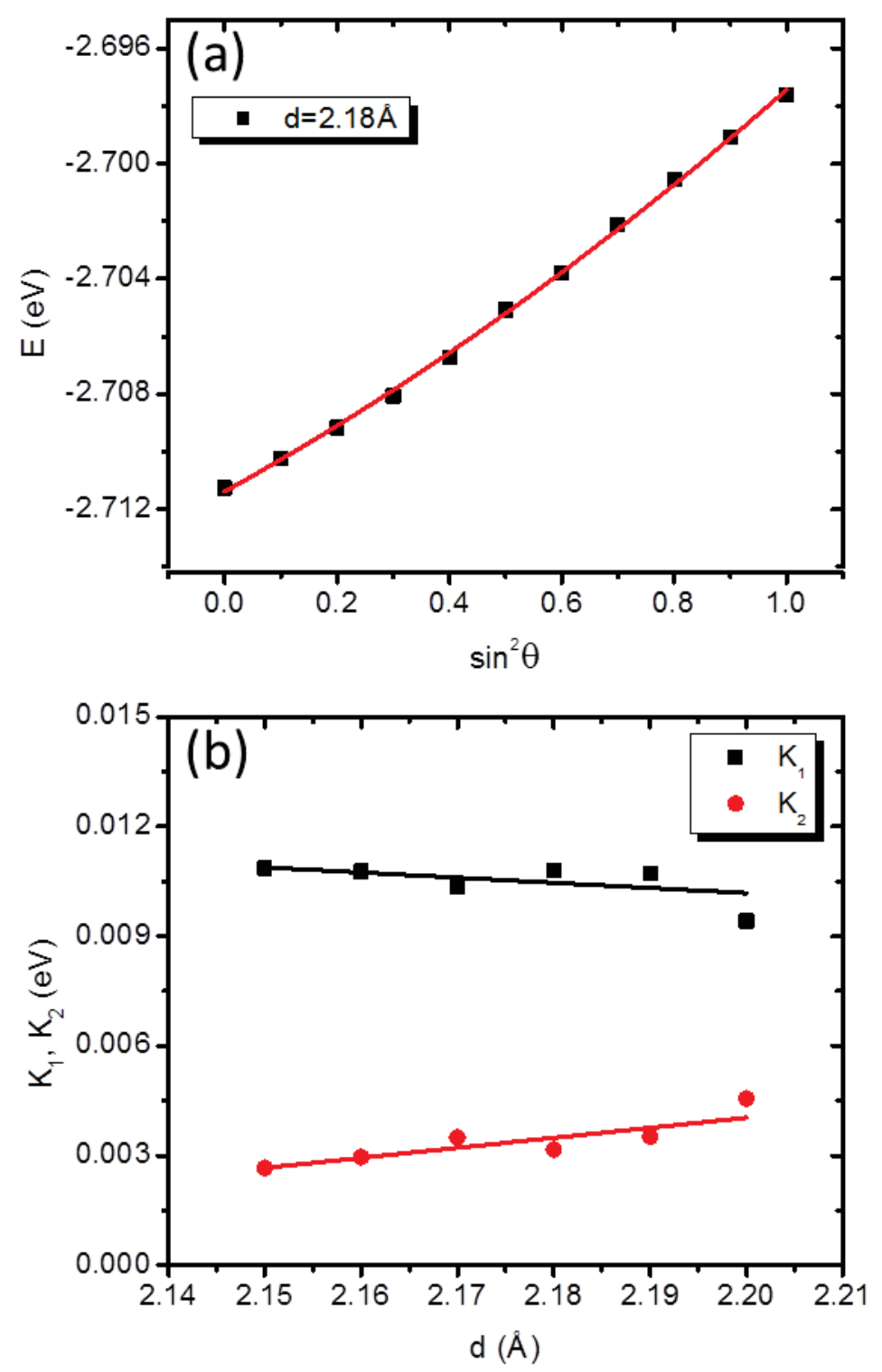

Figure 5.3 (a) Total energies with different spin angles $\theta$ for bond length $d=2.18 \AA$. Red curve represents the fitting for those energies according to Eq. (5.6). (b) The first and second order MAE constants, $K_{1}$ and $K_{2}$, for Ni atomic chain with different bond lengths from $d=2.15 \AA$ to $2.20 \AA$ A. Both sets of constants are linearly fitted vs. $d$. 
For the above MAE calculations, although $\theta$ is varied, the spin moment is almost constant $\left(M_{S 0}=1.135 \mu_{B}\right)$, i.e., the introduction of SOC doesn't affect much on the spin moment. However, since spin moment softening effect always exists in statics and dynamics process, which would quite probably influence the MAE constants, the $M_{S^{-}}$ dependent MAE constants should also be calculated. It has been reported that MAE constant for FePt obeys the rule of $K \propto M_{S}^{2}$ [266], and we assume that the same rule also exists in our $\mathrm{Ni}$ atomic chain system, based on which MAE with constrained spin moment $M_{S}$ has been calculated. Fig. 5.4 shows two sets of energies with varied constrained $M_{S}$ in [100] and [001] directions, respectively, and both of them vary linearly with respect to $M_{S}^{2}$. It can be assumed that such a linearship is also shared by the energies with other $\theta$, and all those energies will converge to the same point for $M_{S}=0$ while restore to the original picture as shown in Fig. 5.3 (a) for $M_{S}=M_{S 0}$. Since our MAE constants are obtained from the energies of varied $\theta$, both $K_{1}$ and $K_{2}$ will obey the same rule in the following form:

$$
K_{1,2}\left(M_{S}\right)=\left(M_{S} / M_{S 0}\right)^{2} K_{1,2}\left(M_{S 0}\right) .
$$

In order to further confirm it, we use the values obtained from Fig. 5.4, $\mathrm{MAE}=E[100]-E[001]=11 \mathrm{meV} \times M_{S}^{2}$, and for $M_{S}=M_{S 0}$, we have $\mathrm{MAE}=14 \mathrm{meV}=$ $K_{1}+K_{2}$, consistent with our previous comments. 


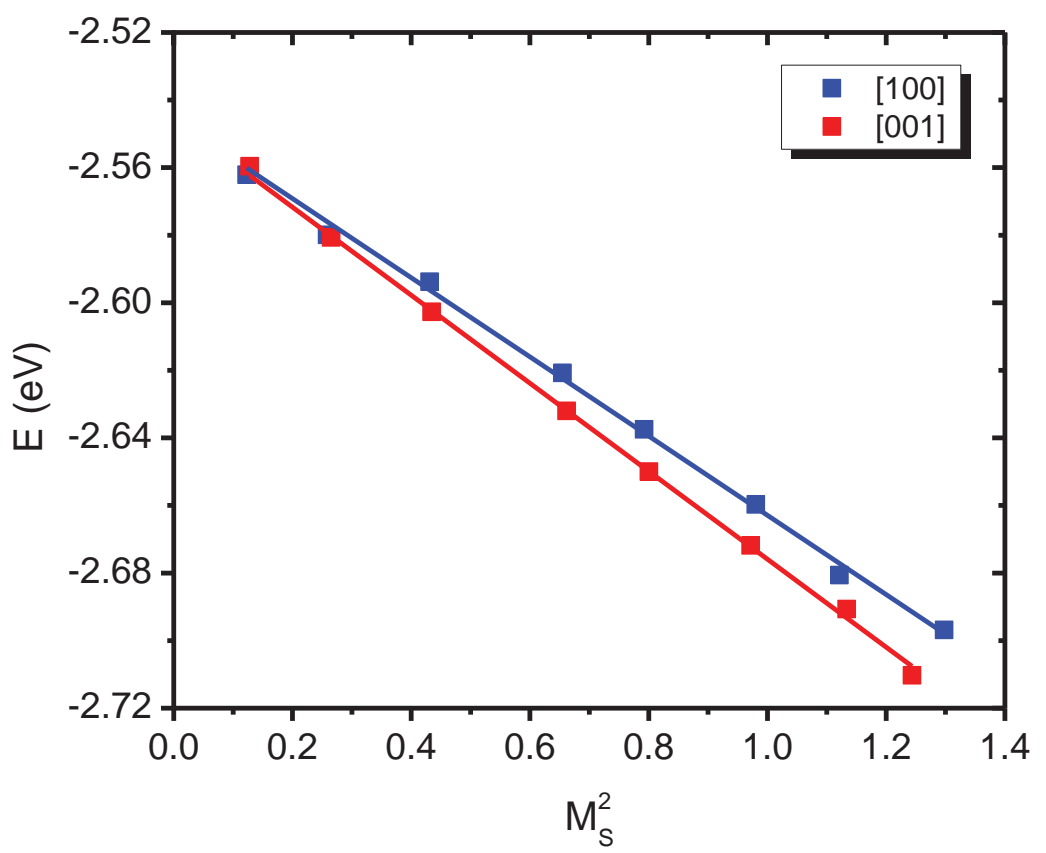

Figure 5.4 Total energies for various constrained spin moments $M_{S}$ which are either perpendicular ([100]) or parallel ([001]) with the atomic chain. Both sets of values are linearly fitted, represented by blue and red curve, respectively.

Ni atomic orbital moments $\mathbf{M}_{L}$ with varied $\theta$ are also obtained, but there are no obvious relationship between $\mathbf{M}_{L}$ and $\theta$ or $\sin ^{2} \theta$. However, evident linear relationship between $\mathbf{M}_{L}$ and spin moment $\mathbf{M}_{S}$ is shown in Fig. 5.5. $\mathbf{M}_{S}^{\perp}\left(\mathbf{M}_{L}^{\perp}\right)$ and $\mathbf{M}_{S}^{\|}\left(\mathbf{M}_{L}^{\|}\right)$are the spin (orbital) moment components perpendicular and parallel with the chain direction, respectively. It is worth noted that the parallel orbital moment component is much larger than the perpendicular component, indicating a strong SOC effect when spin is along the chain direction and the magnitude for orbital moment is comparable with that of spin moment. It is also noted that there are two piecewise linear regions separated by an angle constant around $48.2^{\circ}$, i.e., 


$$
\mathbf{M}_{L}=\left(0.14 M_{S}^{\|}+0.48 \mu_{B}\right) \mathbf{m}^{\|}+0.114 M_{S}^{\perp} \mathbf{m}^{\perp}
$$

for $\theta \leq 48.2^{\circ}$, and

$$
\mathbf{M}_{L}=0.79 M_{S}^{\|} \mathbf{m}^{\|}+\left(0.091 M_{S}^{\perp}+0.019 \mu_{B}\right) \mathbf{m}^{\perp}
$$

for $\theta>48.2^{\circ}$, where $\mathbf{m}^{\|}$and $\mathbf{m}^{\perp}$ only represent the direction parallel and perpendicular with the chain, respectively. Although a good linearship can be achieved for the two regions, the reason for the existence of such a separating angle constant is unclear. Based on the above analysis, it can be expected that the orbital moment within the domain wall will be smaller than that in the domain, resulting in a domain wall with reduced atomic moments even without considering the spin moment softening effect, which will therefore lead to a deviation between the directions of spin moment and atomic moment (the sum of spin and orbital moment).

Although atomic bond length can affect magnetic parameters such as exchange and MAE constants, the exchange interaction and SOC effect can also influence the bond length. For the spin-spiral state with $\mathrm{q} \neq 0$, the equilibrium bond length will deviate from its origin value. Based on our calculation results, new equilibrium bond length of $d \pm 0.005 \AA( \pm 0.2 \%$ of $d)$ for q up to 0.2 has been achieved. For the case considering SOC effect, the difference for the equilibrium bond lengths with spin along [100] and [001] direction is $0.0005 \AA$, i.e., there is a magnetostriction as small as $0.2 \%$. Thus, the equilibrium bond length deviation due to either exchange interaction or SOC is so small that it can be ignored. 

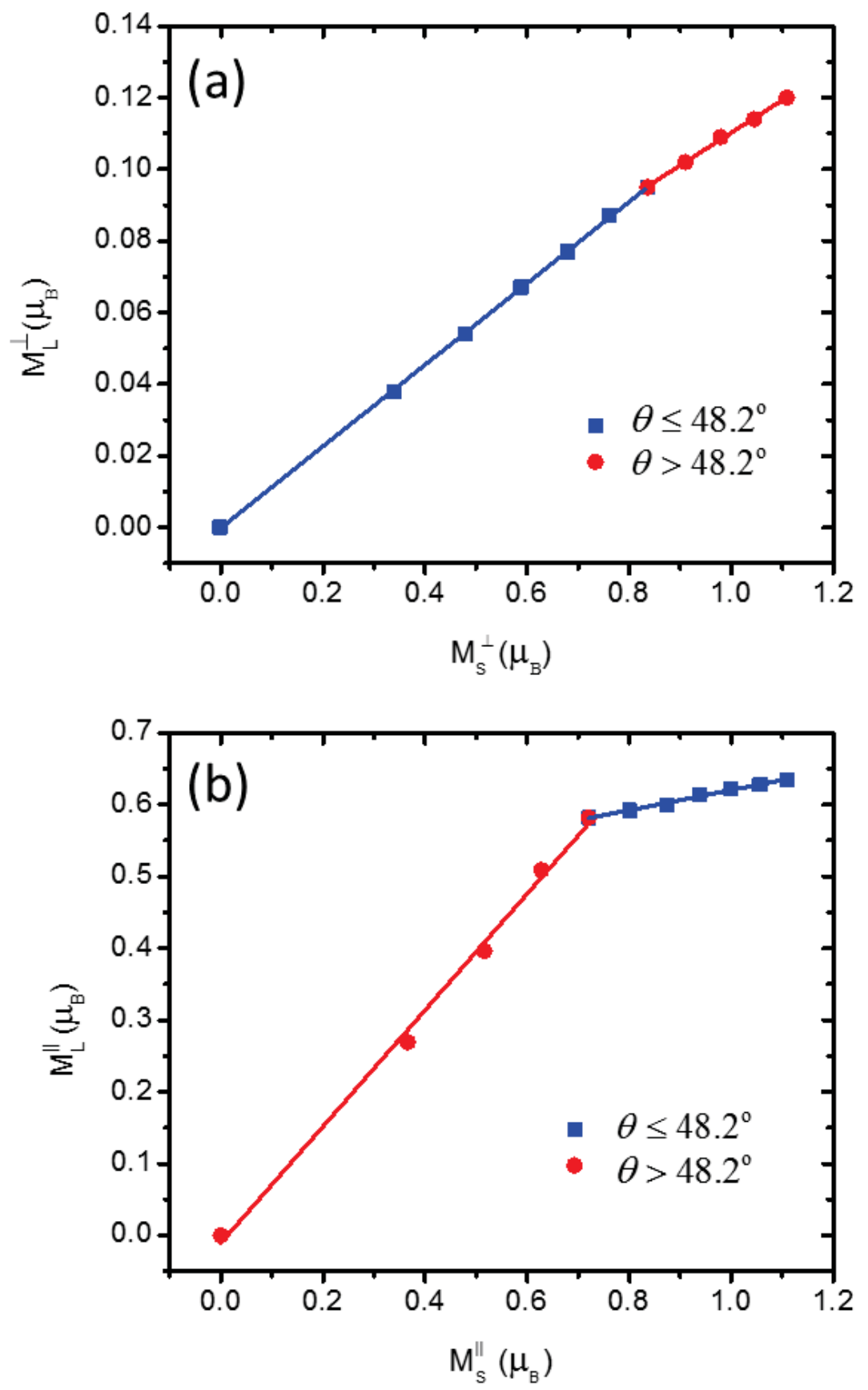

Figure 5.5 Orbital moments vs. spin moments at various spin angles $\theta$, with their components either perpendicular (a) or parallel (b) with the atomic chain direction. Both sets of values are linearly fitted piecewisely by an angle constant $48.2^{\circ}$. 
Based on all the above calculations, we found that the exchange energy, MAE, and orbital moment are all dependent of the spin moment which is determined by the configuration of spin orientation $\mathbf{m}$, and hence $\mathbf{m}$ is the essential order parameter to determine the $\mathrm{Ni}$ atomic chain magnetic system, which might make the LLG equation valid for the atomistic model to be discussed in Sec. III. Since some magnetic parameters might be modified by the temperature effect, the temperature-dependent magnetic parameters should also be calculated.

\subsubsection{Magnetic Parameters at Finite Temperature}

For the temperature dependent of $\mathrm{Ni}$ atomic chain magnetic properties, we adopt the approach employed in Ref. [267]. Based on that approach, the electronic free energy at finite temperature is approximated, and the magnetic parameters such as exchange and MAE constants will be modified from the temperature-dependent electronic free energy, with the atomic moment not significantly changed [236]. The electronic free energy is given by [267]

$$
F(T)=E(0)+\Delta E(T)-T S(T)
$$

where $T$ is the temperature, $E(0)$ is the ab initio calculated energy at zero temperature, $\Delta E(T)$ and $S(T)$ are the temperature dependent of electronic energy difference and electronic entropy, respectively, both of which are caused by the effect of thermally induced electronic excitation. In order to determine the electronic free energy, $\Delta E(T)$ and $S(T)$ should be calculated first in some proper approximations. According to Wolverton and Zunger, there are three levels of approximations (Level 1, 2 and 3) to 203 
consider the energy and entropy associated with electronic excitations [267]. For our ferromagnetic system, we choose Level 2 by which both terms can be determined from $a b$ initio electronic structure calculations, i.e., from the density of states (DOS) which has no explicit temperature dependence. Therefore, the two terms will be

$$
\begin{gathered}
\Delta E(T)=\int_{-\infty}^{+\infty} \varepsilon D(\varepsilon, T=0) f(\varepsilon, T) d \varepsilon-\int_{-\infty}^{\varepsilon_{F}} \varepsilon D(\varepsilon, T=0) d \varepsilon, \\
S(T)=-\int_{-\infty}^{+\infty} D(\varepsilon, T=0)\{f(\varepsilon, T) \ln f(\varepsilon, T)+[1-f(\varepsilon, T)] \ln [1-f(\varepsilon, T)]\} d \varepsilon,
\end{gathered}
$$

where $D(\varepsilon, T=0)$ is the DOS at zero temperature, $\varepsilon_{F}$ the Fermi energy, and $f(\varepsilon, T)$ the Fermi-Dirac distribution $f(\varepsilon, T)=1 /\left(e^{(\varepsilon-\mu) / k T}+1\right)$ with $\mu$ the chemical potential which is dependent of temperature and can be determined by the following condition,

$$
\int_{-\infty}^{+\infty} D(\varepsilon, T=0) f(\varepsilon, T) d \varepsilon=N_{e}=10 .
$$

After determining the chemical potential, the electronic energy difference $\Delta E(T)$, electronic entropy $S(T)$, and finally the electronic free energy $F(T)$ will be obtained.

For the spin-spiral cases, the electronic free energies based on the approximation of Level 2 at different temperatures up to $k_{B} T=100 \mathrm{meV}$ have been calculated and shown in Fig. 5.6 (a). Fig. 5.6 (b) shows the corresponding exchange parameters obtained by fitting the electronic free energies according to Eq. (5.5). It is noted that both the bilinear and biquadratic exchange parameters are decreased with increased temperature, and such a decreasing trend is in good agreement with Ref. [236]. A simple estimation can be made here in order to explain the behavior by introducing $\bar{D}$, an effective average for DOS around $\mu$ within a few meV. Therefore, the estimated results will fall into the 
approximation of Level 3 (Sommerfeld approximation) [267] by giving $\Delta E(T)=\pi^{2} \bar{D} k^{2} T^{2} / 6$ and $T S(T)=\pi^{2} \bar{D} k^{2} T^{2} / 3$. Then, the free energy difference will be $F(T)-E(0)=-\pi^{2} \bar{D} k^{2} T^{2} / 6$, a $T^{2}$-dependent behavior which explains the tiny change for the exchange parameters at low temperatures as shown in Fig. 5.6 (b). For larger q, the hybridization between two spin channels is stronger, and then the DOS around Fermi energy is usually enhanced [104], i.e., $\bar{D}$ is larger for larger q, resulting in a relatively lower free energy for $q>0$ at finite temperatures, which explain why the exchange parameters of finite temperatures are smaller than that of zero temperature and also why these parameters will decrease at higher temperatures.

The electronic free energies for the cases of MAE calculations by the approximation of Level 2 at different temperatures are also calculated as shown in Fig. 5.7 (a), and the corresponding temperature dependent of MAE constants $K_{1}$ and $K_{2}$ obtained by fitting the electronic free energies according to Eq. (5.6) are shown in Fig. 5.7 (b). Like the above exchange parameters, the MAE constants also behave a decreasing trend by increasing the temperature except the case of $k_{B} T=100 \mathrm{meV}$ for which higher MAE orders might be considered. Anyway, it can be concluded that the temperature is destroying the magnetocrystalline anisotropy effect. 

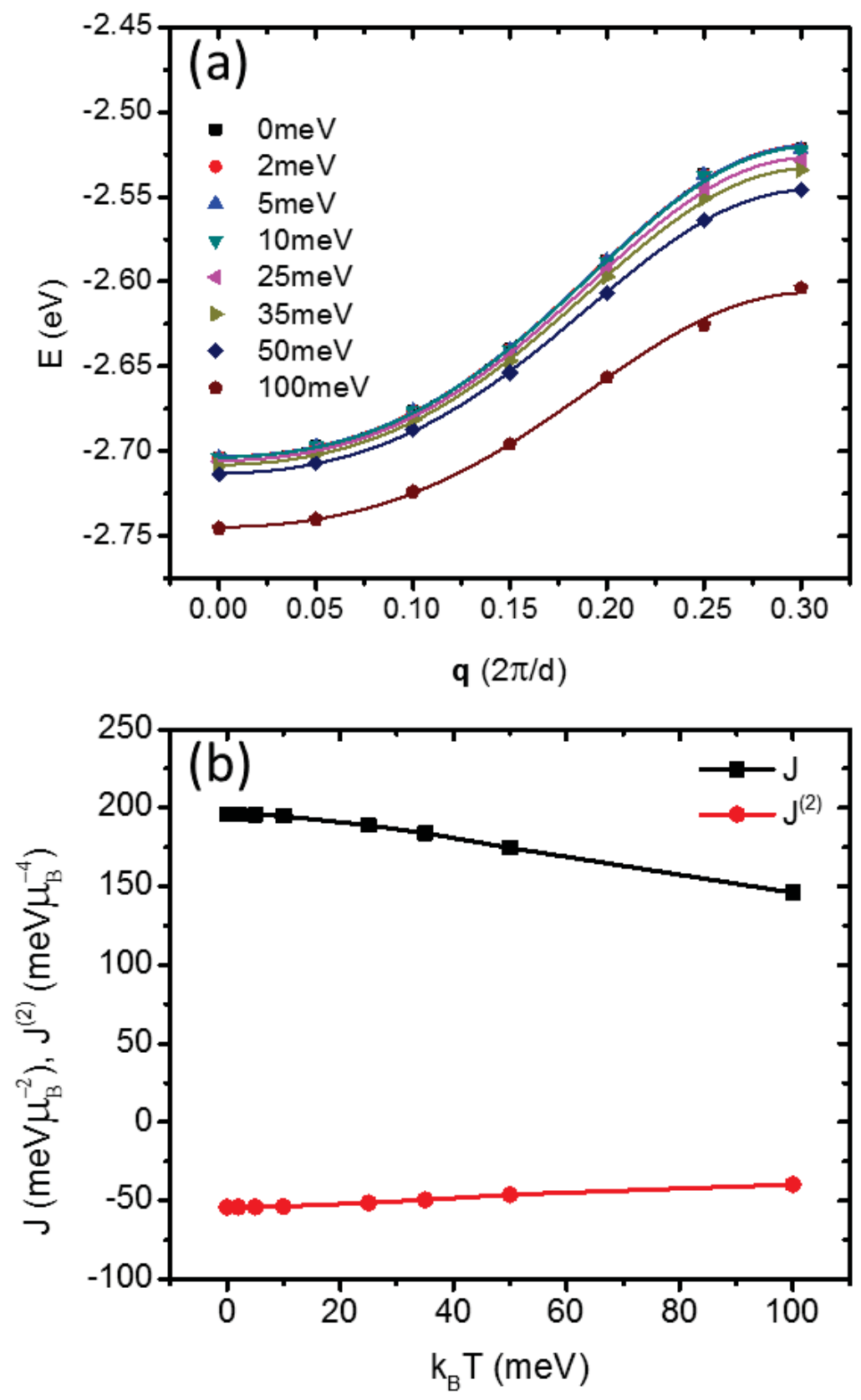

Figure 5.6 (a) Total electronic free energies for spin-spiral state with different propagation q values at different temperatures from 0 to $100 \mathrm{meV}$. Curves represent the fitting according to Eq. (5.5). (b) The bilinear and biquadratic exchange interaction parameters, $J$ and $J^{(2)}$, for Ni atomic chain at different temperatures from 0 to $100 \mathrm{meV}$. 

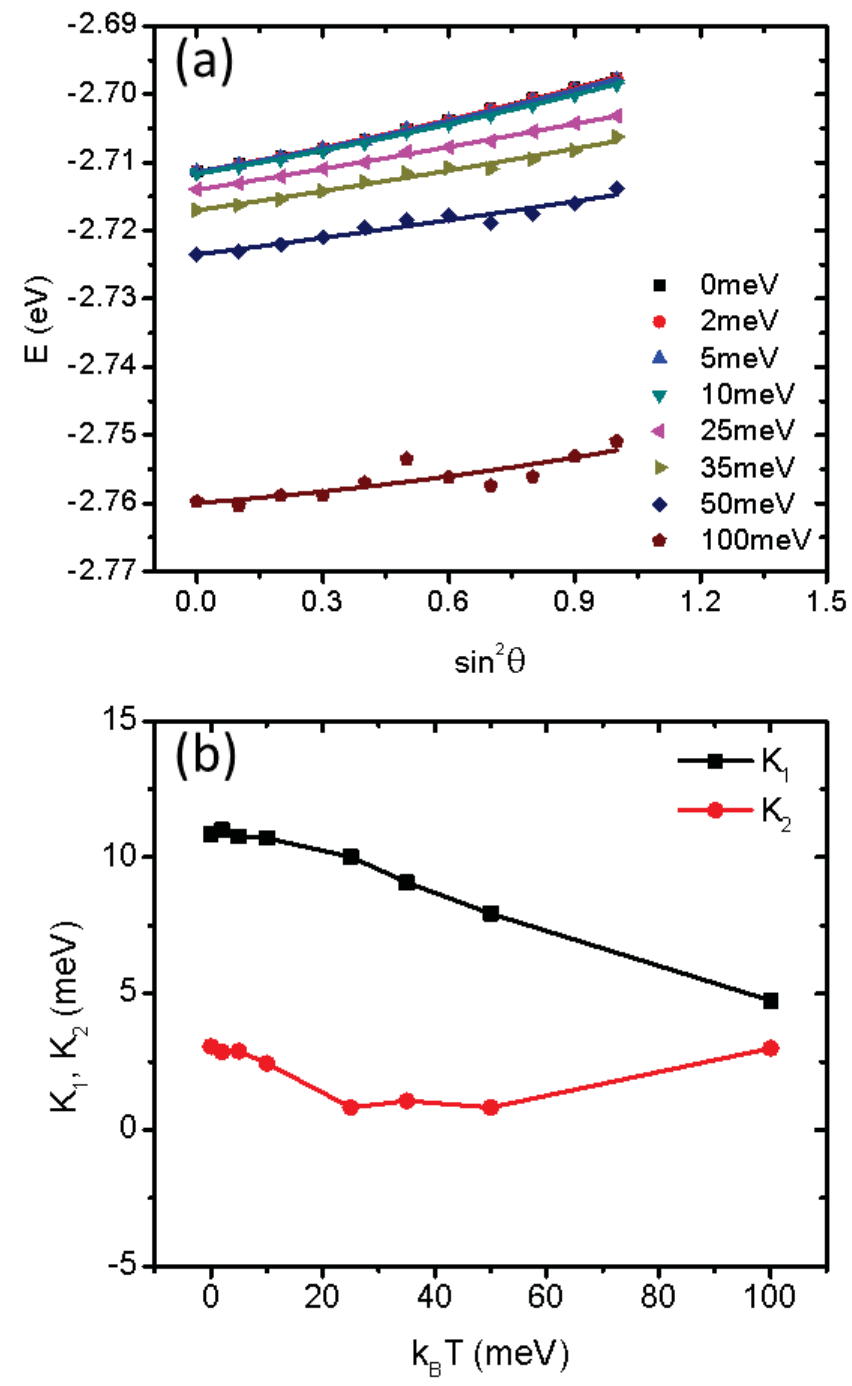

Figure 5.7 (a) Total electronic free energies with different spin angles $\theta$ at different temperatures from 0 to $100 \mathrm{meV}$. Curves represent the fitting according to Eq. (5.6). (b) The first and second order MAE constants, $K_{1}$ and $K_{2}$, for $\mathrm{Ni}$ atomic chain at different temperatures from 0 to $100 \mathrm{meV}$.

For all the above cases, the thermal effect acting on magnetic parameters is considered only in a static or nonvibrating lattice. Since atom oscillations always exist 
due to thermal fluctuations, magnetic parameters will be changing all the time, but the average effect can be roughly approximated. The oscillation amplitude within the temperature range discussed in this paper is about several times of $0.01 \AA$. Actually, due to the linear relationship between magnetic parameters and bond lengths (Fig. 5.2 (b) for exchange interaction parameters and Fig. 5.3 (b) for MAE constants), the average values can be assumed to be those at equilibrium bond length, $d=2.18 \AA$. Thus, the values of magnetic parameters for $\mathrm{Ni}$ atomic chain at equilibrium bond length can also be roughly used as the effective parameters although the thermal induced lattice vibration effect is taken into account, but for the accurate description of lattice vibrations, complex phonon modes should be considered and the spin-phonon interactions will also play a role.

\subsection{Spin Dynamics Simulations}

\subsubsection{Atomistic Model}

Based on the above ab initio studies for Ni monoatomic chain ferromagnetic system, we found that the exchange interactions, the MAE, as well as the orbital moment $\mathbf{M}_{L}$, all of them can be expressed in a function of spin moment $\mathbf{M}_{S}$ with certain coefficients. Besides, the other two Hamiltonian terms considering dipolar interactions and external field, involving both spin and orbital moments, can be also determined only by the single variable $\mathbf{M}_{S}$. Since the spin moment $\mathbf{M}_{S}$ can be obtained from $\mathbf{m}$, the orientations of $\mathbf{M}_{S}$, the total moment for the $i$ th atom in Ni monoatomic chain with length $N$ (number of atoms),

$$
\mathbf{M}_{i}=\mathbf{M}_{S i}+\mathbf{M}_{L i}
$$


will be determined by the configurations of $\mathbf{m}$. As to the orbital moment,

$$
\mathbf{M}_{L i}=0.114 \mathbf{M}_{S i}+\left(0.026 \mathbf{M}_{S i} \cdot \hat{\mathbf{p}}+0.433 M_{S i}\right) \hat{\mathbf{p}}
$$

for $\theta \leq 48.2^{\circ}$ or $\left|\mathbf{m}_{i} \cdot \hat{\mathbf{p}}\right| \geq 0.6665$, and

$$
\mathbf{M}_{L i}=\left(0.091+\frac{0.017}{\sqrt{1-\left(\mathbf{m}_{i} \cdot \hat{\mathbf{p}}\right)^{2}}}\right) \mathbf{M}_{S i}+\left(0.699-\frac{0.017}{\sqrt{1-\left(\mathbf{m}_{i} \cdot \hat{\mathbf{p}}\right)^{2}}}\right)\left(\mathbf{M}_{S i} \cdot \hat{\mathbf{p}}\right) \hat{\mathbf{p}}
$$

for $\theta>48.2^{\circ}$ or $\left|\mathbf{m}_{i} \cdot \hat{\mathbf{p}}\right|<0.6665$. For the spin moment,

$$
\mathbf{M}_{S i}=M_{S i} \mathbf{m}_{i}
$$

Within Eq. (5.15)-(5.17), $\hat{\mathbf{p}}=[100]$ is the chain direction which is also the easy axis in our spin dynamics simulation cases, $\mathbf{m}_{i}$ is the spin orientation for the $i$ th atom, and $M_{S i}$ is the magnitude of spin moment and can be determined according to Eq. (5.1) which can be rewritten as

$$
M_{S i}=M_{0}+\sum_{n=1}^{3} a_{n}\left(\mathbf{m}_{i-n} \cdot \mathbf{m}_{i}+\mathbf{m}_{i} \cdot \mathbf{m}_{i+n}\right)
$$

up to the third nearest atoms. Since $a_{2}$ and $a_{3}$ are very small compared with the other two coefficients according to Fig. 5.1 (b), actually, only the first nearest atoms play an important role in spin moment softening effect.

The total Hamiltonian is composed of four terms given by

$$
H=H_{\text {exch }}+H_{M A E}+H_{d d}+H_{e x t},
$$

and all of the terms can be parametrized based on our ab initio studies in Sec. II. For the exchange interactions, the Heisenberg Hamiltonian $H_{\text {exch }}$ is given by Eq. (5.4) with two 
ab initio calculated exchange parameters $J$ and $J^{(2)}$. The parametrized MAE term $H_{\text {MAE }}$ is described by

$$
H_{M A E}=\left(\frac{M_{S i}}{M_{0}}\right)^{2} \sum_{i}\left\{K_{1}\left[1-\left(\mathbf{m}_{i} \cdot \hat{\mathbf{p}}\right)^{2}\right]+K_{2}\left[1-\left(\mathbf{m}_{i} \cdot \hat{\mathbf{p}}\right)^{2}\right]^{2}\right\}
$$

where $\hat{\mathbf{p}}$ is the easy axis, $K_{1}$ and $K_{2}$ are the two ab initio calculated MAE constants. For the dipole-dipole interaction Hamiltonian, we have

$$
H_{d d}=-\frac{1}{2} \sum_{i \neq j} \mathbf{M}_{i} \hat{\mathbf{Q}}_{i j} \mathbf{M}_{j},
$$

where the elements of $\hat{\mathbf{Q}}_{i j}$ are given by $Q_{i j}^{\mu \nu}=\frac{\mu_{0}}{4 \pi} \frac{3 r_{i j}^{\mu} r_{i j}^{\nu}-r_{i j}^{2} \delta^{\mu \nu}}{r_{i j}^{5}}$ with $r_{i j} \equiv\left|r_{i}-r_{j}\right|$ and $\mu$, $v$ representing $x, y$ and $z$ direction. The fourth one, Zeeman term $H_{e x t}$ is given by

$$
H_{e x t}=-\sum_{i} \mathbf{B}_{e x t} \cdot \mathbf{M}_{i}
$$

where $\mathbf{B}_{\text {ext }}$ is the external magnetic field. It is noted that both the dipolar interaction term and the Zeeman term relate to the total atomic moment directly. At finite temperature, the values shown in Fig. 5.6 (b) and Fig. 5.7 (b) should be used as the exchange and MAE parameters, and for an arbitrary temperature between $k_{B} T=0 \sim 100 \mathrm{meV}$, an interpolation can be made to obtain the corresponding parameters.

The spin dynamics can be described by the evolution of spin orientation $\mathbf{m}_{i}$ which is determined by the stochastic LLG equation [153]

$$
\frac{\partial \mathbf{m}_{i}}{\partial t}=-\tilde{\gamma} \mathbf{m}_{i} \times \mathbf{B}_{i}-\ddot{\gamma} \alpha \mathbf{m}_{i} \times\left(\mathbf{m}_{i} \times \mathbf{B}_{i}\right),
$$


where $\tilde{\gamma}=\gamma /\left[\left(1+\alpha^{2}\right) M_{S 0}\right]$ with $\gamma$ the gyromagnetic ratio, $\alpha$ is the damping parameter, and $\mathbf{B}_{i}$ is the effective local magnetic field including the thermal effect given by

$$
\mathbf{B}_{i}=-\frac{\partial H}{\partial \mathbf{m}_{i}}+h_{i}(t),
$$

where $h_{i}(t)$ is the temperature-dependent random field which is assumed to be a Gaussian stochastic process and fulfills the following statistical properties, [146]

$$
\left\langle h_{i}^{\mu}(t)\right\rangle=0, \quad\left\langle h_{i}^{\mu}(t) h_{i}^{v}\left(t^{\prime}\right)\right\rangle=\sigma^{2} \delta_{i j}^{\mu v} \delta\left(t-t^{\prime}\right)
$$

with $\sigma=\sqrt{2 \alpha k_{B} T / \dot{\gamma}}$. Following Langevin dynamics, the stochastic field introduced here makes the dynamics behaviors satisfy Boltzmann statistics. Since the accurate value of damping parameter $\alpha$ for $\mathrm{Ni}$ atomic chain is unknown, $\alpha=0.1$ is used in this paper and the spin dynamics behavior with other damping parameter values is also discussed in this paper. The time step is $10^{-5} \mathrm{ps}$ for the spin dynamics simulations, which is much lower than the moment relaxation time.

\subsubsection{Magnetic Domain Wall}

As discussed above, a very narrow domain wall is expected due to the large MAE. Now the truly atomic magnetic domain wall structure can be understood by our spin dynamics simulations based on the atomistic model. Fig. 5.8 (a)-(c) show the magnetic atomic moment structure as well as the spin moment structure of a domain wall in an infinite Ni chain at temperature $k_{B} T=0,15$ and $25 \mathrm{meV}$ (room temperature), respectively. Obviously, the domain wall width is only of a few atom-distance and the atomic moment 
'length' within domain wall is smaller than that in the domain region. The domain wall profiles for the atomic moment $\mathbf{M}$, spin moment $\mathbf{M}_{S}$, and orbital moment $\mathbf{M}_{L}$ at zero temperature are shown in Fig. 5.9 (a). It is noted that the atomic moment in the homogenous domain region is larger than that within the domain wall, which is mainly due to the orbital moment, since the orbital component parallel with the chain direction is larger than that perpendicular to the chain direction due to SOC as mentioned in Sec. II. Therefore, the direction for the spin moment will be not exactly the same with that for the atomic moment, especially within the domain wall or under intensive thermal fluctuations, as shown in Fig. 5.8. The angle $\theta$ for the spin moment direction deviated from the chain direction is larger than that for the atomic moment.

(a) $\mathrm{k}_{\mathrm{B}} \mathrm{T}=0$

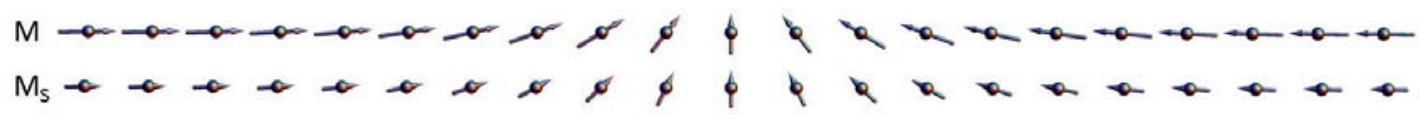

(b) $k_{B} T=15 \mathrm{meV}$

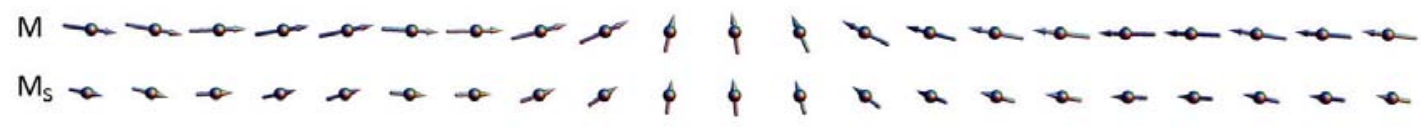

(c) $\mathrm{k}_{\mathrm{B}} \mathrm{T}=25 \mathrm{meV}$

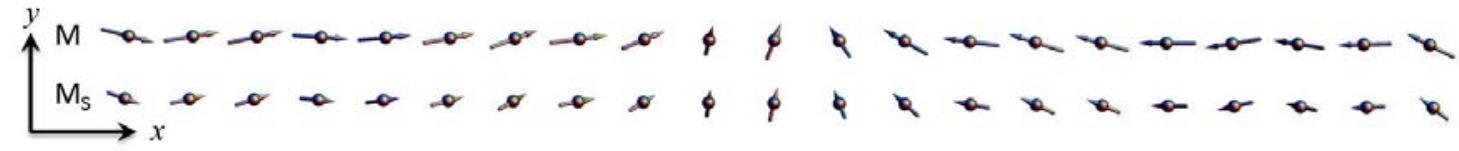

Figure 5.8 The magnetic atomic moment $\mathbf{M}$ as well as the spin moment $\mathbf{M}_{S}$ structure for a domain wall in an infinite $\mathrm{Ni}$ atomic chain at temperature(a) $k_{B} T=0 \mathrm{meV}$, (b) $15 \mathrm{meV}$ and (c) $25 \mathrm{meV}$. 
Usually, the domain wall width can be described by the width parameter $\lambda$ which satisfies the wall profile function as $M_{x} \propto \tanh (x / \lambda)$, as shown by Fig. 5.9 (a) in which the atomic moment component $M_{x}$ is fitted by that function and the corresponding atomic moment domain wall width is defined as $w=2 \lambda_{M}$. However, since the profile for the spin orientations $m_{x}$ shown in Fig. 5.9 (b) is different from that for $M_{x}$, there is a different width parameter $\lambda_{m}$ and hence different domain wall width $w=2 \lambda_{m}$. Both kinds of wall width at various temperatures without including thermal fluctuation field are shown in Fig. 5.9 (c). As expected, the spin orientation width $2 \lambda_{m}$ is larger than the atomic moment width $2 \lambda_{M}$ due to the orbital moment effect discussed above. Both types of wall widths are of around 4 atom-distance with about half an atom-distance between them. Besides, the wall energies are also shown in Fig. 5.9 (c). Usually, the domain wall width and domain wall energy can be roughly approximated as $w=d \sqrt{2 J / K}$ and $E_{D W}=2 \sqrt{2 J K}$, respectively, and thus the slight increase for wall width and the slight decrease for wall energy with increased temperatures as shown in Fig. 5.9 (c) are due to the temperature dependent of exchange parameters and MAE constants as shown in Fig. 5.6 (b) and Fig. 5.7 (b). Both the domain wall width and the domain wall energy are key factors for the thermally induced domain switching process, which will be discussed in Sec. IIIC as following.

Unlike the atomic moment wall width, the spin orientation wall width is directly related to the ballistic magnetoresistance effect which will be more obvious for narrower domain wall, i.e., with smaller $\lambda_{m}$. For a narrow domain wall, the spins vary fast and the 
varying angle between neighbor spins is large. In our case, the largest varying angle for $\mathrm{Ni}$ atomic chain at zero temperature is around $30^{\circ}$, as shown in Fig. 5.9 (b), and usually, the largest spin varying angle within the domain wall can be approximated as $180^{\circ} / \pi \lambda_{m}$. Relatively large magnetoresistance ratio has been reported for $\mathrm{Ni}$ atomic chain with spinspiral domain wall structure with varying angle of $30^{\circ}$ based on theoretical calculations [103], and hence relatively evident ballistic magnetoresistance effect may be expected for our $\mathrm{Ni}$ atomic chain with truly domain wall structure.

Not only the large spin varying angles, but the spin moment softening effect can also enhance the ballistic magnetoresistance effect. At zero temperature, the spin moment at domain wall center is $\sim 3 \%$ reduced. Actually, the spin moment softening is dependent of spin varying angles, as stated in Sec. II. Large spin varying can lead to large softening effect, for example, $180^{\circ}$ spin varying will lead to an almost zero spin moment. Such an effect can be evident when the thermal fluctuations are introduced, and the varying angle can be very large sometimes, which can be found in Fig. 5.8 (b) and (c). For Fig. 5.8 (b), thermal fluctuation field of $k_{B} T=15 \mathrm{meV}$ is applied, the spin varying angle is as large as $50^{\circ}$ at domain wall while $30^{\circ}$ at domain region, leading to a spin moment softening as large as $\sim 8 \%$. For Fig. 5.8 (c), thermal fluctuation field of $k_{B} T=25 \mathrm{meV}$ is applied, the spin varying angle is as large as $80^{\circ}$ at domain wall while $50^{\circ}$ at domain region, leading to a spin moment softening as large as $\sim 20 \%$. According to our atomistic model, such a reduction for spin moment can almost quadratically reduce the exchange interactions, MAE and even the dipolar interactions, while linearly reduce the orbital moment as well as the external field effect. For instance, a $\sim 8 \%$ spin moment softening will lead to a $\sim 16 \%$ reduction for MAE, relatively more evident than the linear effect. Thus, the spin 
moment softening effect will definitely affect the spin dynamics behavior especially under intensive thermal fluctuations.
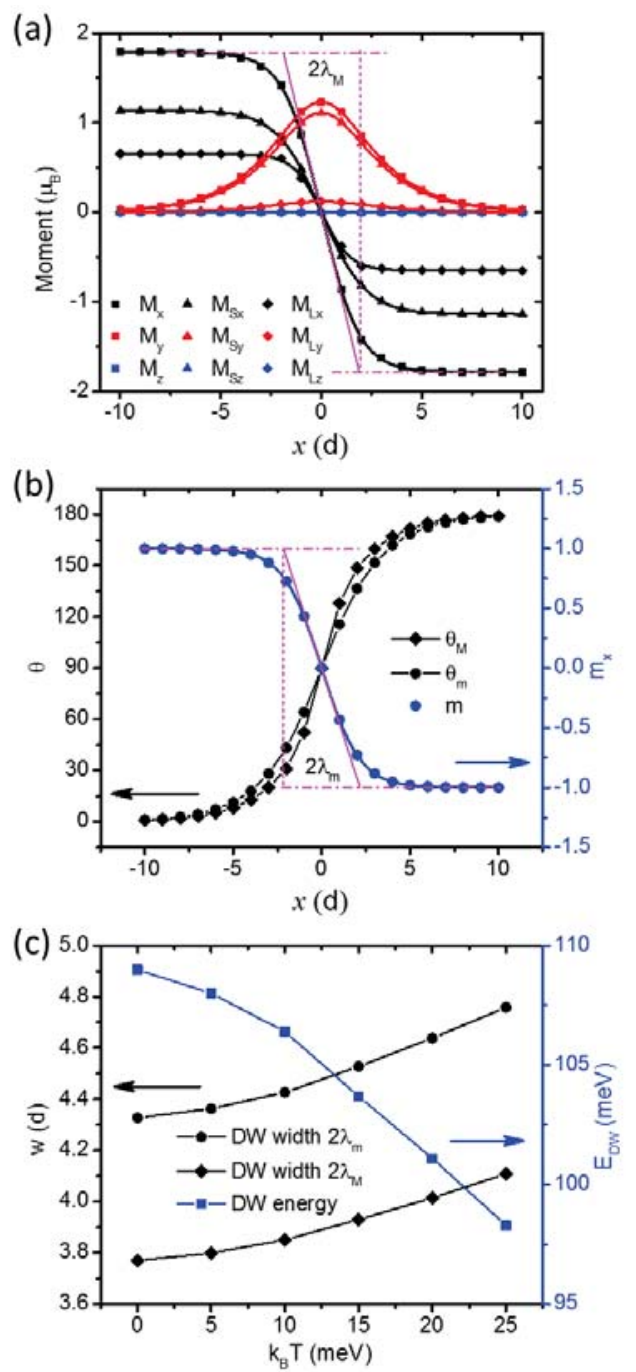

Figure 5.9 (a) The atomic moment $\mathbf{M}$, spin moment $\mathbf{M}_{S}$ and orbital moment $\mathbf{M}_{L}$ components profiles of the domain wall at zero temperature shown in Fig. 5.8 (a). $x$-components are fitted by $\tanh (x / \lambda)$, with $w=2 \lambda_{M}$ corresponding to the atomic moment domain wall width. (b) Spin orientation profile and its fitting curve (blue color), with $w=2 \lambda_{m}$ corresponding to the spin orientation domain wall width. Atomic moment angle $\theta_{M}$ and spin orientation angle $\theta_{m}$ across the domain wall (black color). (c) Spin orientation domain wall widths $2 \lambda_{m}$ and atomic moment domain wall widths $2 \lambda_{M}$ (black color) as well as domain wall energies (blue color) at various temperatures from 0 to $25 \mathrm{meV}$. 


\subsubsection{Magnetic Domain Stability and Switching}

Due to the large MAE, short atomic chain can also maintain ferromagnetic domain very well, and hence the single domain state of Ni monoatomic chains with finite and relatively short length are focused in this section for the spin dynamics study. At zero temperature, all the atomic moments exactly point along the chain direction and will never be switched unless driven by an external field. Once thermal fluctuations are included, those atomic moments, with either the directions or magnitudes, will be

fluctuated, and the domain state can also be switched within a certain time window, but the ferromagnetic state will maintain until the temperature beyond a critical value, $T_{c}$, the Curie temperature. Above the Curie temperature, the ferromagnetic state disappears and the average atomic moment approaches to zero. Thus, the Curie temperature $T_{c}$ for the finite length atomic chain should be determined at first. Fig. 5.10 shows the average atomic moment component $M_{x}$ at different temperatures for a chain of 12-atom length. It is noted that the average moment approaches to zero at $k_{B} T=60 \mathrm{meV}$, indicating that $k_{B} T_{c}$ is around $60 \mathrm{meV}$, i.e., $T_{c} \sim 700 \mathrm{~K}$ for the 12 -atom $\mathrm{Ni}$ chain. As is expected, the Curie temperature for atomic chain with a different length will also be different. Our simulation results show that for the 30 -atom length chain, $T_{c} \sim 460 \mathrm{~K}\left(k_{B} T_{c} \sim 40 \mathrm{meV}\right)$, which is lower than that for the 12-atom chain. Actually, according to the simple theoretical argument [227], the Curie temperature for 1D finite atomic chain can be determined by $T_{c}=2 J /\left(k_{B} \ln (N-1)\right)$, where $N$ is the chain length, demonstrating that shorter chains usually have larger $T_{c}$, in a good agreement with our simulation results. Since all the atomic chains in this section are shorter than 30-atom, they will all maintain 
ferromagnetic state for $k_{B} T<40 \mathrm{meV}$. One can expect that the existence of spin moment softening effect can decrease the Curie temperature in a certain manner. Actually, our simulations results show that for conserved spin moment (without spin moment softening effect) of a 12-atom chain, the Curie temperature is $T_{c} \sim 810 \mathrm{~K}\left(k_{B} T_{c} \sim 70 \mathrm{meV}\right)$, slightly higher than the softening case.

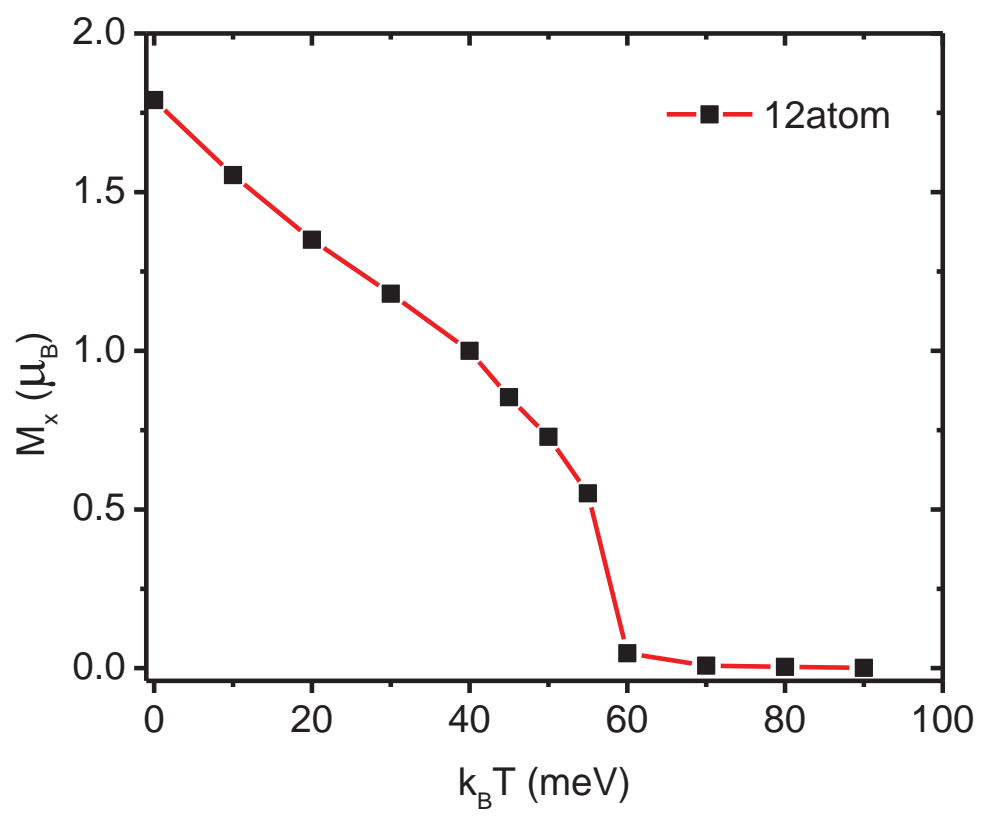

Figure 5.10 Average atomic moment component $M_{x}$ for a chain of 12-atom length at different temperatures.

For finite temperature $T<T_{c}$, domain switching process will happen. Fig. 5.11 (a) shows a serious of switching events for the domain of 12-atom chain under $k_{B} T=15 \mathrm{meV}$ in 1000ps. It is noted that a total of 28 switching events have happened during 1000ps, and hence the average lifetime for each switching is around $\tau \sim 34$ ps. Fig. 5.11 (b) shows 
the detailed dynamics process for the average atomic moment in one single switching event as marked in Fig. 5.11 (a). The switching process is shown to be very fast due to the relative short chain length. What's more, the existence of the aforementioned anisotropic orbital moment effect makes the atomic moment motion trace within an ellipsoid rather than a sphere.

It is expected the lifetime $\tau$ is dependent of both the temperature $T$ and chain length by obeying an Arrhenius law, [253]

$$
\tau=\tau_{0} \exp \left(E_{B} / k_{B} T\right)
$$

where $E_{B}$ is the activation energy barrier and the prefactor $\tau_{0}$ is called the attempt time or attempt period. It is the lifetime $\tau$ that tells the stability of a single ferromagnetic domain state which can be treated as an information carrier. Since $\tau$ is determined by both $E_{B}$ and $\tau_{0}$, those two key factors should be determined by our simulations. The average lifetimes $\tau$ for atomic chains of different lengths from 6- to 26-atom within a temperature range of $k_{B} T=13 \sim 25 \mathrm{meV}$ are calculated from our simulated results. By fitting those values in a linear manner according to Eq. (5.26) as shown by the inset of Fig. 5.12 (a) for a 6-atom chain, both factors can be obtained. Fig. 5.12 (a) and (b) show the fitted values of $E_{B}$ and $\tau_{0}$ for Ni atomic chains with different lengths, respectively. 

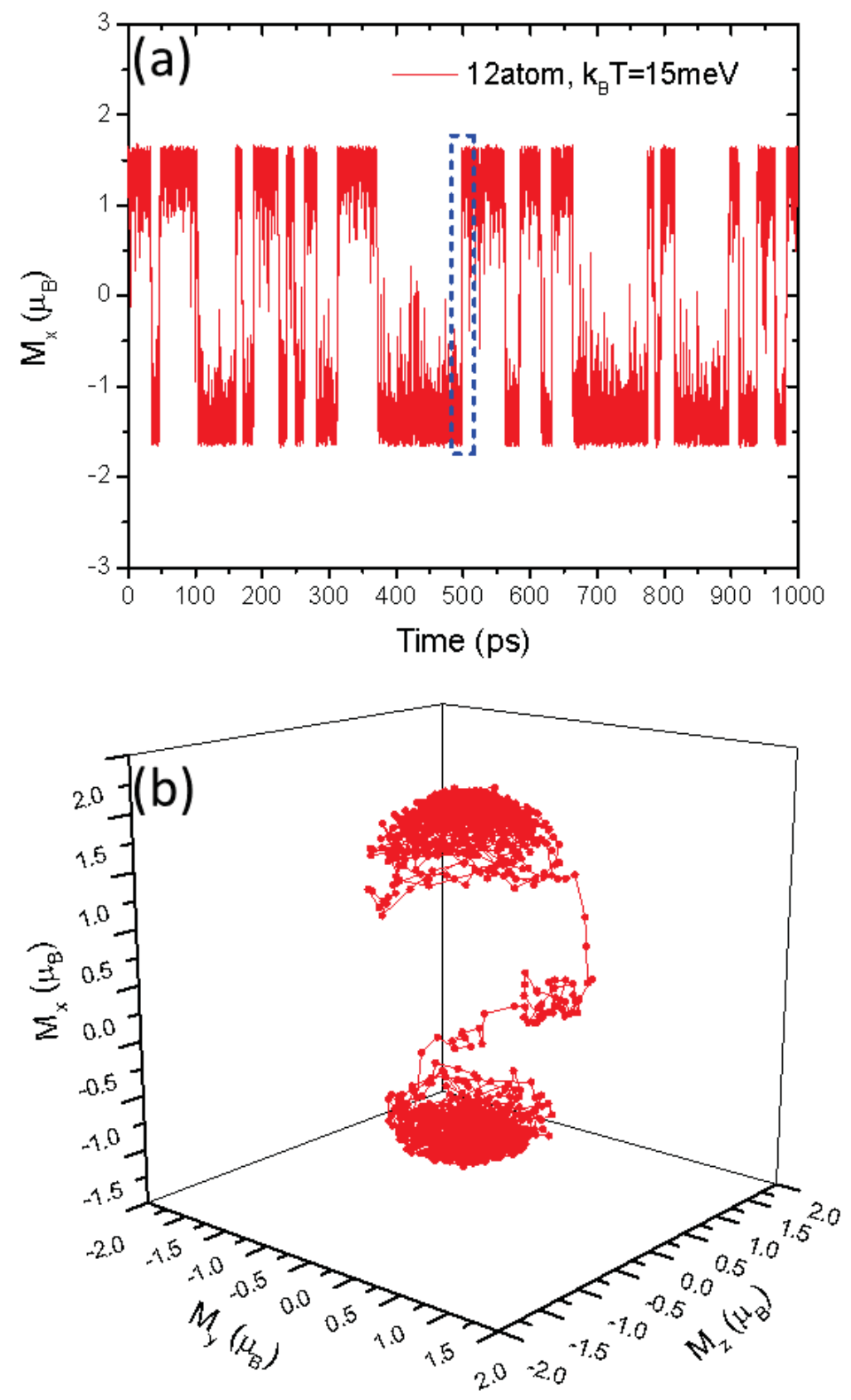

Figure 5.11 (a) Average atomic moment component $M_{x}$ for a chain of 12-atom length at $k_{B} T=15 \mathrm{meV}$ in $1000 \mathrm{ps}$ duration. A total of 28 switching events can be found. (b) Detailed dynamics process for the average atomic moment $\mathbf{M}$ in one single switching event as marked in (a) by the blue dash square. 
For the activation energy $E_{B}$, our simulated results indicate an almost constant value, $\sim 96.3 \mathrm{meV}$, for the chains whose length are no shorter than 14-atom. It has been reported that two regimes exist for the domain switching process [253]. For a chain with the length much smaller than domain wall width (Regime I), the switching is realized by the single domain rotation with the activation energy $E_{B} \approx N K$ which is determined by the MAE and dependent on the chain length $N$. For the chain much longer than domain wall width (Regime II), the switching process is mostly realized by the domain wall motion, for which the domain wall is usually nucleated at the chain edge and then driven by the fluctuating field along the chain to reverse the domain state, with the activation energy the same with domain wall energy $E_{B} \approx E_{D W}$ which is constant and not dependent on the chain length. Of course, the domain wall can also be nucleated in the chain interior, but the activation energy should be doubled since a domain wall pair is required to be nucleated together, which makes the latter case a low-probability event but that must be considered for the infinite chain system [268]. It is not difficult to predict that the activation energy at Regime II will be always larger than that at Regime I. According to Fig. 5.12 (a), since all the chain lengths are larger than the domain wall width which is nearly 4-atom as shown in Fig. 5.9 (c), all the cases we considered are at Regime II, and the activation energy $96.3 \mathrm{meV}$ is also in the vicinity of the domain wall energy which is around $100 \mathrm{meV}$ as shown in Fig. 5.9 (c). Precisely speaking, the domain wall width used to distinguish Regime I and II mustn't be the aforementioned wall width $w=2 \lambda$, but the effective wall width $N_{D W}$ discussed in following content which seems more proper for current discussions. The tiny reducing for the activation energy $96.3 \mathrm{meV}$ with respect to 
the ideal domain wall energy seems due to the fact that the domain wall nucleated at the chain edge is not exactly the same as the ideal one, and the spin moment softening effect might also reduce the domain wall energy a little bit. For the chains with lengths smaller than 14-atom, the activation energies are smaller than the constant value since the chain is not long enough for an intact domain wall, which agrees very well with the results in Ref. [253].

At Regime II, the lifetime is determined by the thermally induced domain wall motion, and once the domain wall propagation process is complete, the switching process is finished. Since it is thought that the domain wall propagates only between $0.5 N_{D W}$ and $N-0.5 N_{D W}$, the attempt time $\tau_{0}$ is proposed to obey a linear rule as [125]

$$
\tau_{0} \propto\left(N-N_{D W}\right)
$$

where $N_{D W}$ is the effective domain wall width. According to Fig. 5.12 (b), such a linearship is observed when the chain length is no less than 14-atom, the same condition for constant $E_{B}$. For chain lengths less than 14-atom, the attempt time is enhanced by decreasing the chain length. It is noted that for the linear part the effective domain wall width is $N_{D W}=8$ atom which is about twice of the actual domain wall width shown by Fig. 5.9 (c), indicating that the effective width required to maintain the nucleated domain wall state without annihilated back at the edge is about 8 -atom which is larger than the commonly defined wall width $2 \lambda \approx 4$ atom. Since both the activation energy $E_{B}$ and attempt time $\tau_{0}$ have been determined, the lifetime $\tau$ for finite chain length and finite temperature therefore can be calculated according to Eq. (5.2). 

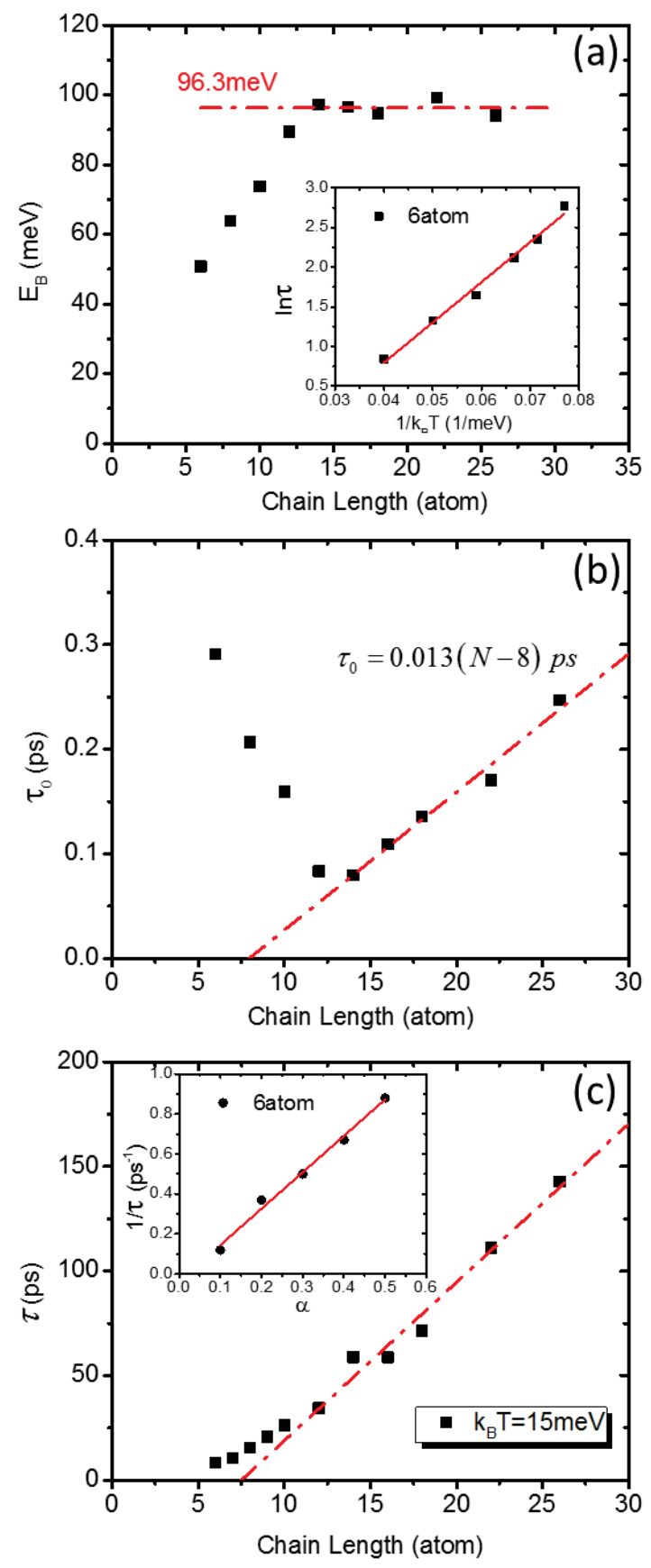

Figure 5.12 (a) Activation energies $E_{B}$ for $\mathrm{Ni}$ atomic chain with various lengths from 6-atom to 26-atom. The inset of (a) shows the linear fitting according to Eq. (5.26) for a 6-atom chain. (b) Attempt times $\tau_{0}$ for $\mathrm{Ni}$ atomic chain with various lengths from 6-atom to 26-atom. The red dashdot line represents a linear fitting for $N \geq 14$. (c) The lifetimes for atomic chains with different lengths at $k_{B} T=15 \mathrm{meV}$. The red dash-dot line represents a linear fitting for $N \geq 14$. The inset of (c) shows a linear fitting between $\tau^{-1}$ and $\alpha$ for a 6-aotm chain at $k_{B} T=15 \mathrm{meV}$. 
Fig. 5.12 (c) shows the lifetimes for atomic chains with different lengths at $k_{B} T=15 \mathrm{meV}$, from which a lifetime enhancement is observed for longer chains. Since $E_{B}$ is constant at a region where the chain length is no less than 14-atom, $\tau \propto \tau_{0}$ is satisfied at that region at constant temperature $k_{B} T=15 \mathrm{meV}$, and hence there is also a linear relationship noted in Fig. 5.12 (c) with the effective wall width also pretty close to 8 -atom. At $k_{B} T=15 \mathrm{meV}$, the thermal fluctuations are so strong that the single domain state lifetime is quite short, in the order of 100ps according to Fig. 5.12 (c). In order for more stable domain state, lower temperature is required. For example, for a chain with 6-atom length, the temperature as low as $T=14 \mathrm{~K}$ is required for the ferromagnetic single domain state staying as long as 10 years, while for a 12-atom chain the corresponding temperature is $T=24 \mathrm{~K}$. One can expect that for ferromagnetic transition-metal atomic chain with smaller MAE, since the activation energy $E_{B}$ will be smaller, the required temperature will be even much lower. All the above results are corresponding to damping parameter $\alpha=0.1$ used in our simulations, however, the actual value of $\alpha$ for $\mathrm{Ni}$ atomic chain is unknown while the lifetime $\tau$ or attempt time $\tau_{0}$ are usually $\alpha$ dependent, so that the relationship between $\tau$ (or $\tau_{0}$ ) and $\alpha$ should be investigated. The inset of Fig. 5.12 (c) shows a linear relationship between $\tau^{-1}$ and $\alpha$ for a 6-aotm chain at $k_{B} T=15 \mathrm{meV}$, i.e., $\tau$ (or $\left.\tau_{0}\right) \propto \alpha^{-1}$. Usually, one can expect the actual value of $\alpha$ is in the range of $(0.01,1.0)$, and then the actual attempt time $\tau_{0}$ will be in the range of $\left(0.1 \tau_{0}, 10 \tau_{0}\right)$ where $\tau_{0}$ is the attempt time corresponding to $\alpha=0.1$ as shown in Fig. 5.12 (b). 

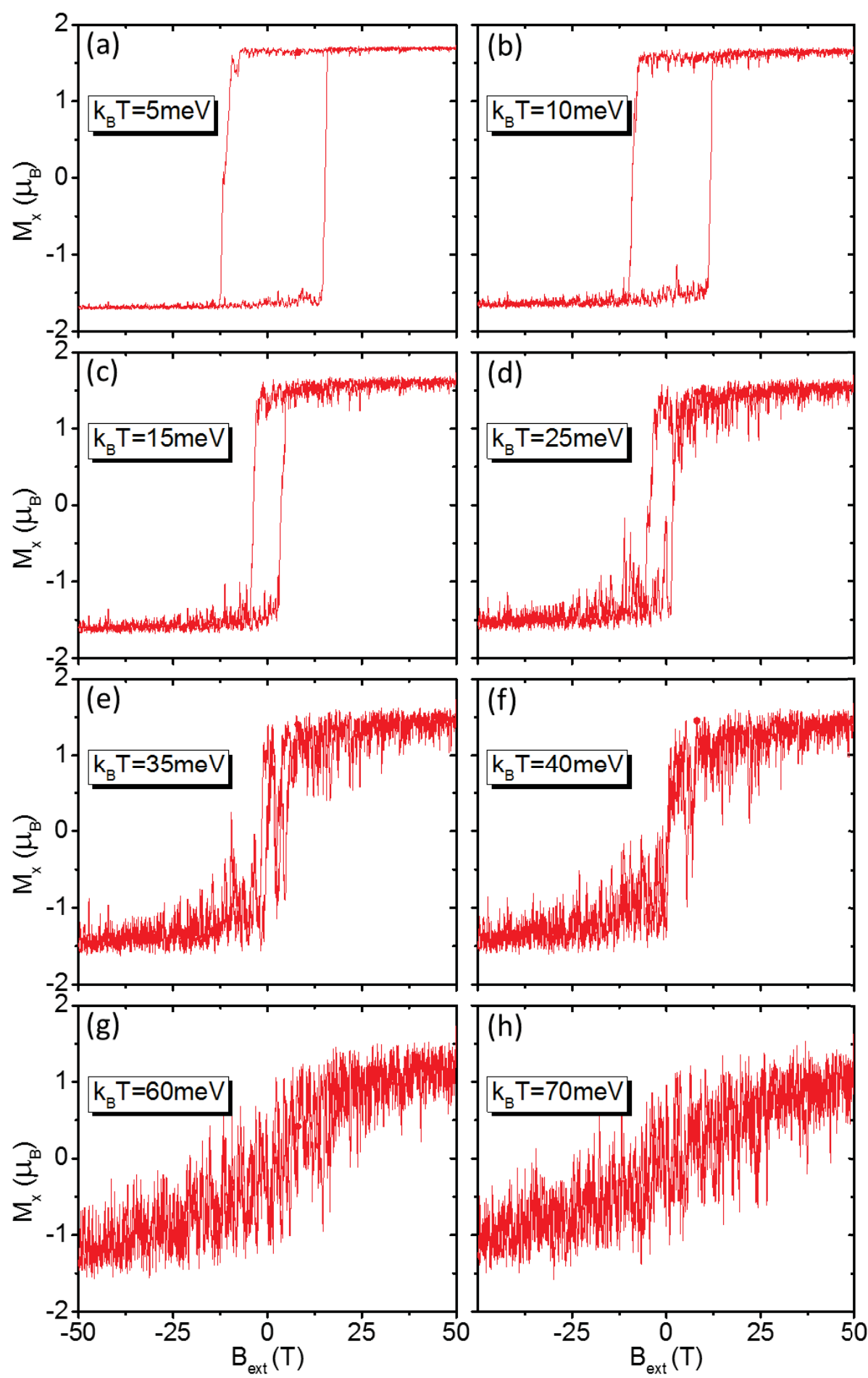

Figure 5.13 M-B hysteresis loops with external field of (-50T, 50T) and measurement period of 60ps for a 12-atom chain at temperatures (a) $k_{B} T=5 \mathrm{meV}$, (b) $10 \mathrm{meV}$, (c) $15 \mathrm{meV}$, (d) $25 \mathrm{meV}$, (e) $35 \mathrm{meV}$, (f) $40 \mathrm{meV}$, (g) $60 \mathrm{meV}$, and (h) $70 \mathrm{meV}$. 
The ferromagnetic single domain switching under external magnetic field is quite essential for the magnetic recording. For finite $\mathrm{Ni}$ atomic chain, it is paramagnetic state above the Curie temperature $T_{c}\left(k_{B} T_{c}=60 \mathrm{meV}\right)$, while below $T_{c}$, the state depends on both the measurement time and temperature. At constant measurement time, there is a blocking temperature $T_{B}$ below which it is ferromagnetic state while above it is superparamagnetic state. A magnetic field loop of $(-50 \mathrm{~T}, 50 \mathrm{~T})$ within a $60 \mathrm{ps}$ period is applied to a 12-atom chain at different temperatures, and all the corresponding hysteresis loops are shown in Fig. 5.13. It is noted that atomic moment fluctuations become more pronounced at higher temperatures. At relatively low temperature, ferromagnetic state maintains at zero external field and the hysteresis loop is very sharp with the coercivity field continuously reduced at increased temperatures until $k_{B} T=35 \mathrm{meV}$ at which the hysteresis disappears (Fig. 5.13 (e)), indicating a transition from the ferromagnetic state to the superparamagnetic state, and that transition temperature is the blocking temperature $k_{B} T_{B}=35 \mathrm{meV}\left(T_{B} \sim 400 \mathrm{~K}\right)$. At temperature between $T_{B}$ and $T_{c}$ (Fig. 5.13 (f)), although there is no hysteresis any more, the ferromagnetic single domain state can also maintain due to the external field and the transition at zero field is still relatively sharp compared with the paramagnetic state shown in Fig. 5.13 (g)-(h) in which the M-B curves are very soft. Those M-B curves for Ni monoatomic chains below and above blocking temperature $T_{B}$ are in good agreement with those for the 50-atom Co monoatomic chain [227], but with a relatively sharp transition manner caused by the larger MAE. Obviously, our simulation results show that higher MAE can help enhance the ferromagnetic single domain stability, increase the operation temperature, and shorten the required chain length. 


\subsection{Conclusion}

In conclusion, freestanding $\mathrm{Ni}$ monoatomic chain with high MAE at finite temperature has been investigated by both $a b$ initio studies and spin dynamics simulations. All the fundamental magnetic parameters, such as exchange interaction parameters, uniaxial MAE constants, and magnetic orbital moments are calculated at $a b$ initio DFT level at zero temperature. Since the spin moment softening effect caused by the hybridization effect between two spin channels are universally existed for itinerant ferromagnets like $\mathrm{Ni}$, the relevant softening coefficients as well as the relationships between spin moment and those fundamental parameters are also investigated. The finite temperature effect is taken into account by employing an approach to the electronic free energy, and the exchange interaction parameters as well as the MAE constants at various finite temperatures are obtained from the temperature-dependent electronic free energies. In order to understand the domain and domain wall properties of $\mathrm{Ni}$ monoatomic chain which are quite essential for 1D nanomagnetism and nanospintronics, an atomistic model using parametrized Hamiltonians which are provided by the above ab initio studies is employed. Our study shows that both the atomic moment and the parametrized Hamiltonian are determined by the spin orientations $\mathbf{m}$ of the $\mathrm{Ni}$ atomic chain, and therefore the whole spin dynamics can be determined by the spin orientations evolution described by the stochastic LLG equation. For the domain wall of Ni atomic chain, our simulation results show that the atomic wall is as narrow as around 4 atom-distance at zero temperature, indicating a relatively evident ballistic magnetoresistance effect. The spin moment softening effect is small at zero temperature, but it can become more pronounced once thermal fluctuations are included, which will significantly soften the 
atomic moment as well as the relevant Hamiltonian like exchange interactions or MAE and hence influence the spin dynamics behaviors like reducing the Curie temperature according to our simulation results. For the ferromagnetic single domain stability and switching, two key factors of the Arrhenius law, the activation energy $E_{B}$ and the attempt time $\tau_{0}$, are quantitatively studied for finite $\mathrm{Ni}$ atomic chain with different lengths. For chains of no less than 14-atom length, $E_{B}$ will be almost constant with the magnitude almost the same with domain wall energy, while $\tau_{0}$ performs a linear relationship with chain length. M-B hysteresis loops at fixed measurement time are given to show the transition between ferromagnetic state and superparamagnetic state separated by the blocking temperature, and both the sharp switching and large coercivity indicate potential applications in magnetic recording. Thus, all the above results show that for $\mathrm{Ni}$ monoatomic chain, the high MAE can help shrink the domain wall size, shorten the required chain length, enhance the ferromagnetic single domain stability, and increase the operation temperature to improve performance in $1 \mathrm{D}$ nanomagnetism and nanospintronics. Our studies can shed a light on other high MAE 1D transition-metal monoatomic chain systems.

\subsection{Future Research}

1. $3 \mathrm{~d}$ or $4 \mathrm{~d}$ transition-metal monoatomic chains in Zigzag from instead of straight form will be taken into account in the future. Their magnetic parameters, especially the MAE, will be calculated based on the ab initio method, while the domain wall structure or spin texture will be investigated based our atomistic model. 
2. If deposited on substrate, the magnetic properties might be very different from those of freestanding systems. Therefore, some monoatomic chain systems with high MAE deposited on certain substrate will be also considered for the future ab initio and spin dynamic studies.

3. Since magnetoresistance for sharp domain wall or fast varying spin textures is a key factor in spintronics, we will use another $a b$ initio program called SMEAGOL to study this topic.

4. Atomic logic element based on single atoms or atomic clusters might be also studied by using our spin dynamics simulations and ab initio calculations. 


\section{Chapter 6. Summary ${ }^{\S}$}

\subsection{Magnetic Domain Walls in Thin Films}

Static and dynamic properties of domain wall with or without substructures in thin films driven by magnetic field or spin-polarized current are focused in Chapter 3. For the case of domain wall without substructures driven by magnetic field, we have investigated the dynamics of one-dimensional domain wall line in ultrathin $\mathrm{Pt} / \mathrm{Co} / \mathrm{Pt}$ film with perpendicular anisotropy in the creep, depinning and flow regimes using our micromagnetic simulations. The creep exponent $\alpha=1.24 \pm 0.05$ is determined by fitting $v \propto \exp \left\{-U_{c}\left(1-H / H_{c}\right)^{\alpha} / k_{B} T\right\}$ for driving field near the depinning force, indicating that the value $\alpha=5 / 4$ based on washboard potential model is supported by our simulation results. A brief analysis based on a phenomenological approach was made for the pinning energies $U_{c}$ in both creep regimes, $H<H_{c}$ and $H \ll H_{c}$, and our analysis indicates a close value for the disorder lengths $u_{0}$ and $\xi$ which are related to two distinct type of pinning potentials. Such a determination for the creep exponent $\alpha$ can also be extended to many other areas in physics.

Since substructures are commonly existed in magnetic domain walls, we also studied the statics and dynamics of domain wall line with substructures in ultrathin magnetic film at finite temperature by micromagnetic simulations. For statics, the roughness exponent $\zeta$ of domain wall line can be slightly decreased by substructures due to the enhanced thermal fluctuations at substructures, and the domain wall line can also

\footnotetext{
${ }_{\S}$ The material contained in Section 6.2 of this chapter was previously partially published in Journal of Applied Physics, 2012. 112(8): p. 083903 and Journal of Magnetism and Magnetic Materials, 2015. 393(0): p. 121-126 by Geng, Liwei D. and Jin, Yongmei M.. See Appendix $\mathrm{C}$ and $\mathrm{D}$ for documentations of permission to republish this material.
} 
be deroughened by substructures mainly due to the increased elasticity. For dynamics, the wall motion behaviors driven by magnetic field in the creep, depinning and flow regimes have been studied. At the steady linear flow regime, the Walker field is increased but the mobility is decreased, both of which are due to the additional motion freedom degree of substructures sliding within the domain wall line by draining the field-induced spin torque at the domain wall, leaving the mobility at the precessional linear flow regime unchanged. At the depinning regime, since one more pinning barrier needs to be overcome due to the slide motion of substructures, the depinning field is increased linearly with the substructure density. At the creep regime for $H<<H_{c}, U_{c 1} / k T$ is reduced, mainly due to the thermal enhancement at substructures, while for $H \leq H_{c}$, the ratios $U_{c 2} / U_{c 1}$ are all around $\sim 0.5$, indicating close disorder lengths in the two field ranges. This work can also be extended to other kinds of elastic strings with substructures.

Like magnetic field, spin-polarized current can also provide a driving force for domain walls, and statics and dynamics properties of domain wall line with an without substructures in thin film with random pinning sites and thermal fluctuations will be also studied, which are our future researches. Before that, current induced substructure motion in ideal magnetic systems without pinning and thermal effect should be studied first. Therefore, current induced Bloch line motion in bulk and thin film with high in-plane uniaxial anisotropy has been investigated by both theoretical calculations and micromagnetic simulations in this section. Both the bulk and thin film systems exhibit almost the same properties for current induced Bloch line motion. The Bloch line velocity 
parallel with current $\mathbf{u}$ is $v_{\|} \approx u$, which is independent of Bloch line types and densities, while the Bloch line velocity perpendicular with $\mathbf{u}$ is $v_{\perp} \approx \chi(\alpha-\beta) u$ for the current applied perpendicular to the domain wall and $v_{\perp} \approx \kappa(\alpha-\beta) u$ for the current parallel with the domain wall, where $\chi$ is more or less inversely proportional to Bloch line density and $\kappa$ is weakly dependent of Bloch line density. Both the theoretical calculations and micromagnetic simulations agree with each other very well. Besides, ultrafast reversal of Bloch line in thin film is also computationally investigated. Our simulation results indicate that Bloch lines in thin film can be ultrafast reversed by magnetic field and spin-polarized current, but with two different mechanisms. Our study on current induced Bloch lines motion as well as the ultrafast Bloch line reversal can shed a light on future Bloch-line based devices in spintronics.

\subsection{Magnetic Domain Walls in Nanowires}

Structures, properties, functionalities as well as some relevant spintronic devices based on field or current driven magnetic domain walls in nanowires are studied or explored in Chapter 4. First, novel nanometer transverse-domain-wall-based logic elements are proposed based on micromagnetic simulations. Unwinding transverse domain walls are generated to avoid the domain wall pile-up effect existed at miniaturized circuits. Since two types of unwinding transverse domain wall pairs can be generated by our novel data generator, new bit definition for ' 1 ' and ' 0 ' is presented accordingly. The logic gates, NOT, AND and OR, are proposed based on the new bit definition and tested by the simulation process. The proposed current/field assistant NOT 
gate performs a no-delay feature. Based on our simulations, more advantages are performed by the nanometer magnetic transverse domain wall logic than the previously proposed submicrometer domain wall logic [34].

Second, $360^{\circ}$ domain walls in planar magnetic nanowires are studied by micromagnetic simulations, and $360^{\circ}$ domain wall generator and shifter register are proposed. It is shown that a nanowire loop with a shape-isotropic wall generator at one end and a shape-anisotropic wall stopper at the other end functions like a data storage stack: $360^{\circ}$ domain walls are generated and pushed into stack under rotating field before overflow while popped out and annihilated when field rotating direction is inverted until underflow. The stack capacity is determined by total nanowire loop length. The $360^{\circ}$ domain wall shift register is constructed by incorporating a shape-isotropic wall generator and a winding nanowire with all the information carriers, $360^{\circ}$ domain walls, stored within the nanowire corners. These simple nanowire structures can be integrated into magnetic circuits as an operation unit for $360^{\circ}$ domain wall generation and storage.

Third, when spin-polarized current is applied to those packed domain walls, an important role of domain wall pile-up in current-driven domain wall depinning in magnetic nanowires is revealed using micromagnetic simulations. It is found that the critical current for domain wall depinning can be substantially reduced and conveniently tuned by controlling domain wall number in the pile-up at pinning site, in analogy to dislocation pile-up responsible for Hall-Petch effect in mechanical strength. Domain wall pinning and depinning at an s-shape bend is considered, and the effects of curvature and current crowding in magnetic circuit on domain wall behaviors are discussed. 
Furthermore, since current induced domain wall depinning and transportation through circular geometry in planar nanowire are crucial for domain-wall-based memory or logic devices, threshold currents for the two processes induced by the conventional spin-transfer torques and the interface-derived spin-orbit torques are theoretically calculated, and those calculated results are also supported by our numerical results based on the 1D model. For spin-orbit torques, the Rashba effect and Spin-Hall effect are taken into account separately for both depinning and transportation cases. Our calculations demonstrate novel means to effectively reduce the threshold current and modulate the current induced transverse DW depinning or transportation process occurred at nanowire circular geometry by Rashba or Spin-Hall effect.

In addition, the magnetic vortex racetrack memory combining both the vortex domain walls realized in nanowire with perpendicular anisotropy and the racetrack conception is proposed using micromagnetic simulations. Racetrack memory and magnetic vortex attract much interest due to the proposed ultrahigh storage density and the distinct static and dynamic properties manifested by its core polarity, respectively. Magnetic vortex racetrack memory (VRM), a combination of both of them, based on densely packed vortices and current induced vortex motion in nanowires, has been proposed to have unique properties different from the domain wall racetrack memory (DWRM). Densely packed vortices in nanowires aroused from the notion of densely packed stripe domains in the thin film with weak perpendicular anisotropy are carried out, and the stability for such vortices as well as their core polarity is studied by our simulations. Two simple designs are demonstrated for the novel VRM, and some of its unique properties, like the current induced vortices transportation behaviors in particular, 
are explored based on our simulation results. Such a VRM may have potential applications in the field of spintronics.

\subsection{Magnetic Domain Walls in Atomic Chains}

Static and dynamic properties of atomic chain are studied by both $a b$ initio calculations and spin dynamic simulations in Chapter 5. Since magnetocrystalline anisotropy energy (MAE) is a key factor in one-dimensional (1D) atomic chain system, freestanding Ni monoatomic chain with high MAE at finite temperature is investigated by both $a b$ initio studies and spin dynamics simulations. All the relevant magnetic parameters are calculated at $a b$ initio density function theory (DFT) level at zero temperature. Magnetic parameters at finite temperature are also calculated based on a DFT approach to the electronic free energy. The spin moment softening effect caused by the hybridization effect between two spin channels are also considered. Based on an atomistic model using parametrized Hamiltonians which are provided by the above $a b$ initio studies, a truly magnetic domain wall structure and the single domain switching process are investigated by our spin dynamics simulations. An atomic domain wall as narrow as 4 atom-distance with slight spin moment softening effect is obtained at zero temperature, which indicates a relatively evident ballistic magnetoresistance effect. For the ferromagnetic single domain switching process, both the activation energy $E_{B}$ and the attempt time $\tau_{0}$ in the Arrhenius law are quantitatively studied for finite $\mathrm{Ni}$ atomic chains with different lengths, and the large $E_{B}$ indicates the strong stability of single domain state, which is also confirmed by the M-B hysteresis loops at fixed measurement 
time by both the sharp switching and large coercivity below the blocking temperature. Our studies can shed a light on other high MAE transition-metal monoatomic chain systems. 


\section{References}

1. Hubert, A. and R. Schafer, Magnetic Domains: The Analysis of Magnetic Microstructures, 2000.

2. Nakatani, Y., A. Thiaville, and J. Miltat, Faster magnetic walls in rough wires. Nat Mater, 2003. 2(8): p. 521-523.

3. Li, Z. and S. Zhang, Domain-Wall Dynamics and Spin-Wave Excitations with Spin-Transfer Torques. Physical Review Letters, 2004. 92(20): p. 207203.

4. Saitoh, E., et al., Current-induced resonance and mass determination of a single magnetic domain wall. Nature, 2004. 432(7014): p. 203-206.

5. Tatara, G. and H. Kohno, Theory of Current-Driven Domain Wall Motion: Spin Transfer versus Momentum Transfer. Physical Review Letters, 2004. 92(8): p. 086601 .

6. Yamaguchi, A., et al., Real-Space Observation of Current-Driven Domain Wall Motion in Submicron Magnetic Wires. Physical Review Letters, 2004. 92(7): p. 077205.

7. Yamanouchi, M., et al., Current-induced domain-wall switching in a ferromagnetic semiconductor structure. Nature, 2004. 428(6982): p. 539-542.

8. Zhang, S. and Z. Li, Roles of Nonequilibrium Conduction Electrons on the Magnetization Dynamics of Ferromagnets. Physical Review Letters, 2004. 93 (12): p. 127204.

9. Barnes, S.E. and S. Maekawa, Current-Spin Coupling for Ferromagnetic Domain Walls in Fine Wires. Physical Review Letters, 2005. 95(10): p. 107204.

10. Beach, G.S.D., et al., Dynamics of field-driven domain-wall propagation in ferromagnetic nanowires. Nat Mater, 2005. 4(10): p. 741-744.

11. Ravelosona, D., et al., Nanometer Scale Observation of High Efficiency Thermally Assisted Current-Driven Domain Wall Depinning. Physical Review Letters, 2005. 95(11): p. 117203.

12. Hayashi, M., et al., Influence of Current on Field-Driven Domain Wall Motion in Permalloy Nanowires from Time Resolved Measurements of Anisotropic Magnetoresistance. Physical Review Letters, 2006. 96(19): p. 197207.

13. Hayashi, M., et al., Dependence of Current and Field Driven Depinning of Domain Walls on Their Structure and Chirality in Permalloy Nanowires. Physical Review Letters, 2006. 97(20): p. 207205.

14. Thomas, L., et al., Oscillatory dependence of current-driven magnetic domain wall motion on current pulse length. Nature, 2006. 443(7108): p. 197-200.

15. Yamanouchi, M., et al., Velocity of Domain-Wall Motion Induced by Electrical Current in the Ferromagnetic Semiconductor (Ga,Mn)As. Physical Review Letters, 2006. 96(9): p. 096601.

16. Hayashi, M., et al., Direct observation of the coherent precession of magnetic domain walls propagating along permalloy nanowires. Nat Phys, 2007. 3(1): p. 21-25.

17. Meier, G., et al., Current-induced domain-wall motion in permalloy semi rings. Journal of Magnetism and Magnetic Materials, 2007. 316(2): p. e966-e968. 
18. Beach, G.S.D., M. Tsoi, and J.L. Erskine, Current-induced domain wall motion. Journal of Magnetism and Magnetic Materials, 2008. 320(7): p. 1272-1281.

19. Hayashi, M., et al., Current-Controlled Magnetic Domain-Wall Nanowire Shift Register. Science, 2008. 320(5873): p. 209-211.

20. Moriya, R., et al., Probing vortex-core dynamics using current-induced resonant excitation of a trapped domain wall. Nat Phys, 2008. 4(5): p. 368-372.

21. Parkin, S.S.P., M. Hayashi, and L. Thomas, Magnetic Domain-Wall Racetrack Memory. Science, 2008. 320(5873): p. 190-194.

22. Youngman, J., et al., Current-induced domain wall nucleation and its pinning characteristics at a notch in a spin-valve nanowire. Nanotechnology, 2009. 20(12): p. 125401.

23. Bader, S.D. and S.S.P. Parkin, Spintronics. Annual Review of Condensed Matter Physics, 2010. 1(1): p. 71-88.

24. Burrowes, C., et al., Non-adiabatic spin-torques in narrow magnetic domain walls. Nat Phys, 2010. 6(1): p. 17-21.

25. Jiang, X., et al., Enhanced stochasticity of domain wall motion in magnetic racetracks due to dynamic pinning. Nat Commun, 2010. 1: p. 25.

26. Thomas, L., et al., Dynamics of Magnetic Domain Walls Under Their Own Inertia. Science, 2010. 330(6012): p. 1810-1813.

27. Junya, S., T. Gen, and K. Hiroshi, A brief review of field- and current-driven domain-wall motion. Journal of Physics D: Applied Physics, 2011. 44(38): p. 384004.

28. Koyama, T., et al., Observation of the intrinsic pinning of a magnetic domain wall in a ferromagnetic nanowire. Nat Mater, 2011. 10(3): p. 194-197.

29. Miron, I.M., et al., Fast current-induced domain-wall motion controlled by the Rashba effect. Nat Mater, 2011. 10(6): p. 419-423.

30. Haazen, P.P.J., et al., Domain wall depinning governed by the spin Hall effect. Nat Mater, 2013. 12(4): p. 299-303.

31. O'Brien, L., et al., Tunable Remote Pinning of Domain Walls in Magnetic Nanowires. Physical Review Letters. 106(8).

32. Allwood, D.A., et al., Submicrometer Ferromagnetic NOT Gate and Shift Register. Science, 2002. 296(5575): p. 2003-2006.

33. Atkinson, D., et al., Magnetic domain-wall dynamics in a submicrometre ferromagnetic structure. Nat Mater, 2003. 2(2): p. 85-7.

34. Allwood, D.A., et al., Magnetic domain-wall logic. Science, 2005. 309(5741): p. 1688-1692.

35. Lewis, E.R., et al., Fast domain wall motion in magnetic comb structures. Nat Mater, 2010. 9(12): p. 980-983.

36. Vanhaverbeke, A., A. Bischof, and R. Allenspach, Control of Domain Wall Polarity by Current Pulses. Physical Review Letters, 2008. 101(10).

37. Miron, I.M., et al., Current-driven spin torque induced by the Rashba effect in a ferromagnetic metal layer. Nat Mater, 2010. 9(3): p. 230-4.

38. Miron, I.M., et al., Fast current-induced domain-wall motion controlled by the Rashba effect. Nat Mater, 2011. 10(6): p. 419-23. 
39. Ryu, K.S., et al., Chiral spin torque at magnetic domain walls. Nat Nanotechnol, 2013. 8(7): p. 527-33.

40. Choi, S., et al., Strong Radiation of Spin Waves by Core Reversal of a Magnetic Vortex and Their Wave Behaviors in Magnetic Nanowire Waveguides. Physical Review Letters, 2007. 98(8): p. 087205.

41. Guslienko, K.Y., K.-S. Lee, and S.-K. Kim, Dynamic Origin of Vortex Core Switching in Soft Magnetic Nanodots. Physical Review Letters, 2008. 100(2): p. 027203.

42. Hertel, R., et al., Ultrafast Nanomagnetic Toggle Switching of Vortex Cores. Physical Review Letters, 2007. 98(11): p. 117201.

43. Janutka, A., Externally driven transformations of vortex textures in flat submicrometer magnets. Physical Review B, 2012. 85(18): p. 184421.

44. Kamionka, T., et al., Magnetic Antivortex-Core Reversal by Circular-Rotational Spin Currents. Physical Review Letters, 2010. 105(13): p. 137204.

45. Kläui, M., et al., Current-induced vortex nucleation and annihilation in vortex domain walls. Applied Physics Letters, 2006. 88(23): p. -.

46. Zhang, Y., et al., Perpendicular-magnetic-anisotropy CoFeB racetrack memory. Journal of Applied Physics, 2012. 111(9): p. -.

47. Muratov, C.B. and V.V. Osipov, Bit Storage by $360^{\circ}$ Domain Walls in Ferromagnetic Nanorings. Magnetics, IEEE Transactions on, 2009. 45(8): p. 3207-3209.

48. Taguchi, K., J.-i. Ohe, and G. Tatara, Ultrafast Magnetic Vortex Core Switching Driven by the Topological Inverse Faraday Effect. Physical Review Letters, 2012. 109(12): p. 127204.

49. Bayer, C., et al. Phase shift of spin waves traveling through a 180\&deg; Bloch domain wall. in Magnetics Conference, 2005. INTERMAG Asia 2005. Digests of the IEEE International. 2005.

50. Hertel, R., W. Wulfhekel, and J. Kirschner, Domain-Wall Induced Phase Shifts in Spin Waves. Physical Review Letters, 2004. 93(25): p. 257202.

51. Thiaville, A., et al., Micromagnetic understanding of current-driven domain wall motion in patterned nanowires. EPL (Europhysics Letters), 2005. 69(6): p. 990.

52. Xiao, J., A. Zangwill, and M.D. Stiles, Spin-transfer torque for continuously variable magnetization. Physical Review B, 2006. 73(5): p. 054428.

53. Li, Z. and S. Zhang, Domain-wall dynamics driven by adiabatic spin-transfer torques. Physical Review B, 2004. 70(2): p. 024417.

54. Thiaville, A., et al., Domain wall motion by spin-polarized current: $a$ micromagnetic study. Journal of Applied Physics, 2004. 95(11): p. 7049-7051.

55. Waintal, X. and M. Viret, Current-induced distortion of a magnetic domain wall. EPL (Europhysics Letters), 2004. 65(3): p. 427.

56. Carva, K. and I. Turek, Ab initio theory of spin-transfer torques. Journal of Magnetism and Magnetic Materials, 2007. 316(2): p. e926-e929.

57. He, J., Z. Li, and S. Zhang, Current-driven vortex domain wall dynamics by micromagnetic simulations. Physical Review B, 2006. 73(18): p. 184408.

58. Hiroshi Kohno, G.T., Junya Shibata, Microscopic Calculation of Spin Torques in Disordered Ferromagnets. Journal of the Physical Society of Japan, 2006. 75(11). 
59. Berkov, D.V. and J. Miltat, Spin-torque driven magnetization dynamics: Micromagnetic modeling. Journal of Magnetism and Magnetic Materials, 2008. 320(7): p. 1238-1259.

60. Jung, S.-W., et al., Current-induced domain wall motion in a nanowire with perpendicular magnetic anisotropy. Applied Physics Letters, 2008. 92(20): p. -.

61. Gen TATARA, T.T., Hiroshi KOHNO, Junya SHIBATA, and Y.N.a.H. FUKUYAMA, Threshold Current of Domain Wall Motion under Extrinsic Pinning, b-Term and Non-Adiabaticity. Journal of the Physical Society of Japan, 2006. 75(6).

62. Dresselhaus, G., Spin-Orbit Coupling Effects in Zinc Blende Structures. Physical Review, 1955. 100(2): p. 580-586.

63. Dyakonov, M.I. and V.I. Perel, Current-induced spin orientation of electrons in semiconductors. Physics Letters A, 1971. 35(6): p. 459-460.

64. Stöhr, J. and H.C. Siegmann, Magnetism: From Fundamentals to Nanoscale Dynamics. 2006.

65. Gambardella, P. and I.M. Miron, Current-induced spin-orbit torques. Philos Trans A Math Phys Eng Sci, 2011. 369(1948): p. 3175-97.

66. Tsutsui, K., et al., Spin Currents Induced by Nonuniform Rashba-Type Spin-Orbit Field. Journal of the Physical Society of Japan, 2011. 80(8): p. 084701.

67. Khvalkovskiy, A.V., et al., Matching domain-wall configuration and spin-orbit torques for efficient domain-wall motion. Physical Review B, 2013. 87(2).

68. Avci, C.O., et al., Fieldlike and antidamping spin-orbit torques in as-grown and annealed Ta/CoFeB/MgO layers. Physical Review B, 2014. 89(21).

69. Martinez, E., S. Emori, and G.S.D. Beach, Current-driven domain wall motion along high perpendicular anisotropy multilayers: The role of the Rashba field, the spin Hall effect, and the Dzyaloshinskii-Moriya interaction. Applied Physics Letters, 2013. 103(7): p. 072406.

70. Liu, L., et al., Spin-torque switching with the giant spin Hall effect of tantalum. Science, 2012. 336(6081): p. 555-8.

71. Emori, S., et al., Current-driven dynamics of chiral ferromagnetic domain walls. Nat Mater, 2013. 12(7): p. 611-6.

72. Haazen, P.P., et al., Domain wall depinning governed by the spin Hall effect. Nat Mater, 2013. 12(4): p. 299-303.

73. Jungwirth, T., J. Wunderlich, and K. Olejnik, Spin Hall effect devices. Nat Mater, 2012. 11(5): p. 382-90.

74. Ryu, J., K.-J. Lee, and H.-W. Lee, Current-driven domain wall motion with spin Hall effect: Reduction of threshold current density. Applied Physics Letters, 2013. 102(17): p. 172404.

75. Perez, N., et al., Chiral magnetization textures stabilized by the DzyaloshinskiiMoriya interaction during spin-orbit torque switching. Applied Physics Letters, 2014. 104(9): p. 092403.

76. Seo, S.-M., et al., Current-induced motion of a transverse magnetic domain wall in the presence of spin Hall effect. Applied Physics Letters, 2012. 101(2): p. 022405 . 
77. Moriya, T., New Mechanism of Anisotropic Superexchange Interaction. Physical Review Letters, 1960. 4(5): p. 228-230.

78. Bode, M., et al., Atomic spin structure of antiferromagnetic domain walls. Nat Mater, 2006. 5(6): p. 477-481.

79. Uchida, M., et al., Real-Space Observation of Helical Spin Order. Science, 2006. 311(5759): p. 359-361.

80. Bode, M., et al., Chiral magnetic order at surfaces driven by inversion asymmetry. Nature, 2007. 447(7141): p. 190-193.

81. Levy, P.M. and S. Zhang, Resistivity due to Domain Wall Scattering. Physical Review Letters, 1997. 79(25): p. 5110-5113.

82. Gregg, J.F., et al., Giant Magnetoresistive Effects in a Single Element Magnetic Thin Film. Physical Review Letters, 1996. 77(8): p. 1580-1583.

83. Hong, K. and N. Giordano, Approach to mesoscopic magnetic measurements. Physical Review B, 1995. 51(15): p. 9855-9862.

84. Tatara, G. and H. Fukuyama, Resistivity due to a Domain Wall in Ferromagnetic Metal. Physical Review Letters, 1997. 78(19): p. 3773-3776.

85. Ebels, U., et al., Spin Accumulation and Domain Wall Magnetoresistance in 35 nm Co Wires. Physical Review Letters, 2000. 84(5): p. 983-986.

86. $\mathrm{Xu}, \mathrm{Y} . \mathrm{B}$., et al., Magnetoresistance of a domain wall at a submicron junction. Physical Review B, 2000. 61(22): p. R14901-R14904.

87. Çetin, B. and N. Giordano, Domain wall resistance in narrow Co wires. physica status solidi (b), 2004. 241(10): p. 2410-2414.

88. Lepadatu, S. and Y.B. Xu, Direct Observation of Domain Wall Scattering in Patterned $\mathrm{Ni}_{80} \mathrm{Fe}_{20}$ and $\mathrm{Ni}$ Nanowires by Current-Voltage Measurements. Physical Review Letters, 2004. 92(12): p. 127201.

89. Tang, H.X., et al., Negative intrinsic resistivity of an individual domain wall in epitaxial (Ga,Mn)As microdevices. Nature, 2004. 431(7004): p. 52-56.

90. van Gorkom, R.P., A. Brataas, and G.E.W. Bauer, Negative Domain Wall Resistance in Ferromagnets. Physical Review Letters, 1999. 83(21): p. 44014404.

91. Elefant, D. and R. Schäfer, Giant negative domain wall resistance in iron. Physical Review B, 2010. 82(13).

92. García, N., M. Muñoz, and Y.W. Zhao, Magnetoresistance in excess of $200 \%$ in Ballistic Ni Nanocontacts at Room Temperature and 100 Oe. Physical Review Letters, 1999. 82(14): p. 2923-2926.

93. Tatara, G., et al., Domain Wall Scattering Explains 300\% Ballistic Magnetoconductance of Nanocontacts. Physical Review Letters, 1999. 83(10): p. 2030-2033.

94. Chung, S.H., et al., Universal Scaling of Ballistic Magnetoresistance in Magnetic Nanocontacts. Physical Review Letters, 2002. 89(28).

95. Chung, S.H., et al., Universal Scaling of Ballistic Magnetoresistance in Magnetic Nanocontacts. Physical Review Letters, 2002. 89(28): p. 287203.

96. Sullivan, M.R., et al., Ballistic magnetoresistance in nickel single-atom conductors without magnetostriction. Physical Review B, 2005. 71(2): p. 024412. 
97. Tatara, G., Domain Wall Resistance Based on Landauer's Formula. J. Phys. Soc. Jpn., 2000. 69(9).

98. Faleev, S.V., et al., Ab initio tight-binding LMTO method for nonequilibrium electron transport in nanosystems. Physical Review B, 2005. 71(19): p. 195422.

99. Sabirianov, R.F., et al., Domain-wall magnetoresistance of Co nanowires. Physical Review B, 2005. 72(5): p. 054443.

100. Burton, J.D., et al., Magnetic Moment Softening and Domain Wall Resistance in Ni Nanowires. Physical Review Letters, 2006. 97(7): p. 077204.

101. García, N., et al., Ballistic magnetoresistance in a magnetic nanometer sized contact: An effective gate for spintronics. Applied Physics Letters, 2001. 79(27): p. 4550.

102. Chopra, H.D., et al., The quantum spin-valve in cobalt atomic point contacts. Nat Mater, 2005. 4(11): p. 832-837.

103. Velev, J. and W.H. Butler, Domain-wall resistance in metal nanocontacts. Physical Review B, 2004. 69(9): p. 094425.

104. Burton, J., et al., Magnetic Moment Softening and Domain Wall Resistance in Ni Nanowires. Physical Review Letters, 2006. 97(7): p. 077204.

105. Yavorsky, B.Y., et al., Giant magnetoresistance due to a domain wall in Fe:Ab initiostudy. Physical Review B, 2002. 66(17).

106. Durgun, E., et al., Nanospintronic properties of carbon-cobalt atomic chains. Europhys. Lett., 2006. 73(4): p. 642-648.

107. Stavros, A., et al., Giant magnetoresistance of nickel-contacted carbon nanotubes. Journal of Physics: Condensed Matter, 2007. 19(4): p. 042201.

108. Durgun, E., et al., Spintronic properties of carbon-based one-dimensional molecular structures. Physical Review B, 2006. 74(23).

109. Czerner, M., B.Y. Yavorsky, and I. Mertig, Magnetic order in geometrically constrained domain walls. Journal of Applied Physics, 2008. 103(7): p. 07F304.

110. Mavropoulos, P., N. Papanikolaou, and P.H. Dederichs, Korringa-Kohn-Rostoker Green-function formalism for ballistic transport. Physical Review B, 2004. 69(12).

111. Bagrets, A., N. Papanikolaou, and I. Mertig, Magnetoresistance of atomic-sized contacts: Anab initiostudy. Physical Review B, 2004. 70(6).

112. Tung, J.C. and G.Y. Guo, Systematic ab initio study of the magnetic and electronic properties of all 3d transition metal linear and zigzag nanowires. Physical Review B, 2007. 76(9): p. 094413.

113. Dag, S., et al., Half-metallic properties of atomic chains of carbon-transitionmetal compounds. Physical Review B, 2005. 72(15).

114. Graham, B.D.C.a.C.D., Introduction to Magnetic Materials (Wiley, New York, 2009).

115. Moore, T.A., et al., Scaling of spin relaxation and angular momentum dissipation in permalloy nanowires. Physical Review B, 2009. 80(13): p. 132403.

116. Bamzai, A.S. and B.M. Deb, The role of single-particle density in chemistry. Reviews of Modern Physics, 1981. 53(1): p. 95-126.

117. Parr, R.G., Density Functional Theory. Annual Review of Physical Chemistry, 1983. 34(1): p. 631-656. 
118. Callaway, J. and N.H. March, Density Functional Methods:Theory and Applications, in Solid State Physics, D.T. Henry Ehrenreich and S. Frederick, Editors. 1984, Academic Press. p. 135-221.

119. Jones, R.O. and O. Gunnarsson, The density functional formalism, its applications and prospects. Reviews of Modern Physics, 1989. 61(3): p. 689-746.

120. Martin, R.M., Electronic Structure: Basic Theory and Practical Methods. 2008.

121. Sholl, D. and J.A. Steckel, Density Functional Theory: A Practical Introduction 2009.

122. Giustino, F., Materials Modelling using Density Functional Theory: Properties and Predictions. 2014.

123. Kresse, G. and J. Hafner, Ab initio molecular dynamics for open-shell transition metals. Physical Review B, 1993. 48(17): p. 13115-13118.

124. Kresse, G. and J. Furthmüller, Efficiency of ab-initio total energy calculations for metals and semiconductors using a plane-wave basis set. Computational Materials Science, 1996. 6(1): p. 15-50.

125. Hobbs, D., G. Kresse, and J. Hafner, Fully unconstrained noncollinear magnetism within the projector augmented-wave method. Physical Review B, 2000. 62(17): p. 11556-11570.

126. Sandratskii, L.M., Symmetry analysis of electronic states for crystals with spiral magnetic order. I. General properties. Journal of Physics: Condensed Matter, 1991. 3(44): p. 8565.

127. Sandratskii, L.M., Symmetry analysis of electronic states for crystals with spiral magnetic order. II. Connection with limiting cases. Journal of Physics: Condensed Matter, 1991. 3(44): p. 8587.

128. Blatter, G., et al., Vortices in high-temperature superconductors. Reviews of Modern Physics, 1994. 66(4): p. 1125-1388.

129. Fukuyama, H. and P.A. Lee, Dynamics of the charge-density wave. I. Impurity pinning in a single chain. Physical Review B, 1978. 17(2): p. 535-541.

130. Paruch, P., T. Giamarchi, and J.M. Triscone, Domain Wall Roughness in Epitaxial Ferroelectric PbZr ${ }_{0.2} \mathrm{Ti}_{0.8} \mathrm{O}_{3}$ Thin Films. Physical Review Letters, 2005. 94(19): p. 197601.

131. Tybell, T., et al., Domain Wall Creep in Epitaxial Ferroelectric $\mathrm{Pb}\left(\mathrm{Zr}_{0.2} \mathrm{Ti}_{0.8}\right) \mathrm{O}_{3}$ Thin Films. Physical Review Letters, 2002. 89(9): p. 097601.

132. Bustingorry, S., A.B. Kolton, and T. Giamarchi, Thermal rounding of the depinning transition in ultrathin Pt/Co/Pt films. Physical Review B, 2012. 85(21): p. 214416.

133. Krusin-Elbaum, L., et al., Stable ultrahigh-density magneto-optical recordings using introduced linear defects. Nature, 2001. 410(6827): p. 444-446.

134. Metaxas, P.J., et al., Creep and Flow Regimes of Magnetic Domain-Wall Motion in Ultrathin Pt/Co/Pt Films with Perpendicular Anisotropy. Physical Review Letters, 2007. 99(21): p. 217208.

135. Lemerle, S., et al., Domain Wall Creep in an Ising Ultrathin Magnetic Film. Physical Review Letters, 1998. 80(4): p. 849-852. 
136. Shibauchi, T., et al., Deroughening of a $1 D$ Domain Wall in an Ultrathin Magnetic Film by a Correlated Defect. Physical Review Letters, 2001. 87(26): p. 267201.

137. Bauer, M., et al., Deroughening of Domain Wall Pairs by Dipolar Repulsion. Physical Review Letters, 2005. 94(20): p. 207211.

138. Chauve, P., T. Giamarchi, and P. Le Doussal, Creep and depinning in disordered media. Physical Review B, 2000. 62(10): p. 6241-6267.

139. Müller, M., D.A. Gorokhov, and G. Blatter, Velocity-force characteristics of a driven interface in a disordered medium. Physical Review B, 2001. 63(18): p. 184305.

140. Bustingorry, S., A.B. Kolton, and T. Giamarchi, Thermal rounding exponent of the depinning transition of an elastic string in a random medium. Physical Review E, 2012. 85(2): p. 021144.

141. Nattermann, T., V. Pokrovsky, and V.M. Vinokur, Hysteretic Dynamics of Domain Walls at Finite Temperatures. Physical Review Letters, 2001. 87(19): p. 197005.

142. Anderson, P.W., Theory of Flux Creep in Hard Superconductors. Physical Review Letters, 1962. 9(7): p. 309-311.

143. Büttiker, M. and R. Landauer, Nucleation theory of overdamped soliton motion. Physical Review A, 1981. 23(3): p. 1397-1410.

144. Nowak, U. and K.D. Usadel, Influence of temperature on the depinning transition of driven interfaces. EPL (Europhysics Letters), 1998. 44(5): p. 634.

145. Duemmer, O. and W. Krauth, Critical exponents of the driven elastic string in a disordered medium. Physical Review E, 2005. 71(6): p. 061601.

146. Brown, W.F., Jr., Thermal Fluctuations of a Single-Domain Particle. Physical Review, 1963. 130(5): p. 1677-1686.

147. Thomson, T., G. Hu, and B.D. Terris, Intrinsic Distribution of Magnetic Anisotropy in Thin Films Probed by Patterned Nanostructures. Physical Review Letters, 2006. 96(25): p. 257204.

148. Martys, N., M. Cieplak, and M.O. Robbins, Critical phenomena in fluid invasion of porous media. Physical Review Letters, 1991. 66(8): p. 1058-1061.

149. Kim, K.-J., et al., Interdimensional universality of dynamic interfaces. Nature, 2009. 458(7239): p. 740-742.

150. Lee, J.-C., et al., Universality Classes of Magnetic Domain Wall Motion. Physical Review Letters, 2011. 107(6): p. 067201.

151. Eduardo, M., The stochastic nature of the domain wall motion along high perpendicular anisotropy strips with surface roughness. Journal of Physics: Condensed Matter, 2012. 24(2): p. 024206.

152. Kolton, A.B., A. Rosso, and T. Giamarchi, Creep Motion of an Elastic String in a Random Potential. Physical Review Letters, 2005. 94(4): p. 047002.

153. Hubert, A. and R. Schäfer, Magnetic Domains: The Analysis of Magnetic Microstructures. 1998: Springer.

154. Martinez, E., et al., Thermal effects in domain wall motion: Micromagnetic simulations and analytical model. Physical Review B, 2007. 75(17): p. 174409. 
155. Jung, S.-W., et al., Current-induced domain wall motion in a nanowire with perpendicular magnetic anisotropy. Applied Physics Letters, 2008. 92(20): p. 202508-3.

156. Kolton, A.B., A. Rosso, and T. Giamarchi, Nonequilibrium Relaxation of an Elastic String in a Random Potential. Physical Review Letters, 2005. 95(18): p. 180604.

157. Bryan, M.T., J. Dean, and D.A. Allwood, Dynamics of stress-induced domain wall motion. Physical Review B, 2012. 85(14): p. 144411.

158. Le Maho, Y., J.-V. Kim, and G. Tatara, Spin-wave contributions to currentinduced domain wall dynamics. Physical Review B, 2009. 79(17): p. 174404.

159. Lisfi, A., et al., Reorientation of magnetic anisotropy in epitaxial cobalt ferrite thin films. Physical Review B, 2007. 76(5): p. 054405.

160. Prejbeanu, I.L., et al., Observation of asymmetric Bloch walls in epitaxial Co films with strong in-plane uniaxial anisotropy. Applied Physics Letters, 2000. 77(19): p. 3066-3068.

161. Schneider, M., S. Müller-Pfeiffer, and W. Zinn, Magnetic force microscopy of domain wall fine structures in iron films. Journal of Applied Physics, 1996. 79(11): p. 8578.

162. Basterfield, J., Domain Structure and the Influence of Growth Defects in Single Crystals of Yttrium Iron Garnet. Journal of Applied Physics, 1968. 39(12): p. 5521.

163. Vlasko-Vlasov, V.K., 1.M. Dedukh, and V.I. Nikitenko, Domain structure of yttrium iron garnet single crystals. Zh. Eksp. Teor. Fiz., 1976. 71: p. 2291-2304.

164. Schippan, F., et al., Magnetic structure of epitaxially grown MnAs on GaAs(001). Journal of Applied Physics, 2000. 88(5): p. 2766.

165. Engel-Herbert, R., et al., Understanding the submicron domain structure of MnAs thin films on GaAs(001): Magnetic force microscopy measurements and simulations. Applied Physics Letters, 2004. 84(7): p. 1132.

166. Kim, J., H. Akinaga, and J. Kim, Direct observation of the spin configurations of vertical Bloch line. Applied Physics Letters, 2011. 98(5): p. 052510.

167. Konishi, S., A new-ultra-density solid state memory: Bloch line memory. Magnetics, IEEE Transactions on, 1983. 19(5): p. 1838-1840.

168. Humphrey, F.B. and J.C. Wu, Vertical bloch line memory. Magnetics, IEEE Transactions on, 1985. 21(5): p. 1762-1766.

169. Engemann, J. Vertical Bloch line memory: state of the art and future prospect. in CompEuro '89., 'VLSI and Computer Peripherals. VLSI and Microelectronic Applications in Intelligent Peripherals and their Interconnection Networks', Proceedings. 1989.

170. Cheynis, F., et al., Controlled Switching of Néel Caps in Flux-Closure Magnetic Dots. Physical Review Letters, 2009. 102(10).

171. Yamada, K., et al., Electrical switching of the vortex core in a magnetic disk. Nat Mater, 2007. 6(4): p. 270-273.

172. Lee, K.-S. and S.-K. Kim, Conceptual design of spin wave logic gates based on a Mach-Zehnder-type spin wave interferometer for universal logic functions. Journal of Applied Physics, 2008. 104(5): p. 053909-4. 
173. Schneider, T., et al., Realization of spin-wave logic gates. Applied Physics Letters, 2008. 92(2): p. 022505-3.

174. Parkin, S.S., M. Hayashi, and L. Thomas, Magnetic domain-wall racetrack memory. Science, 2008. 320(5873): p. 190-4.

175. Kläui, M., et al., Direct Observation of Domain-Wall Configurations Transformed by Spin Currents. Physical Review Letters, 2005. 95(2): p. 026601.

176. Ishaque, Z., et al., Manipulating domain wall chirality by current pulses in Permalloy/Ir nanostrips. arXiv preprint arXiv:1312.4918, 2013.

177. Klein, J.O., et al., VHDL simulation of magnetic domain wall logic. Ieee Transactions on Magnetics, 2006. 42(10): p. 2754-2756.

178. Jin, Y.M., et al., Magnetic structure and hysteresis in hard magnetic nanocrystalline film: Computer simulation. Journal of Applied Physics, 2002. 92(10): p. 6172-6181.

179. Geng, L.D. and Y.M. Jin, Generation and storage of $360^{\circ}$ domain walls in planar magnetic nanowires. Journal of Applied Physics, 2012. 112(8): p. -

180. Himeno, A., et al., Dynamics of a magnetic domain wall in magnetic wires with an artificial neck. Journal of Applied Physics, 2003. 93(10): p. 8430-8432.

181. Smith, D.O. and K.J. Harte, Noncoherent Switching in Permalloy Films. Journal of Applied Physics, 1962. 33(4): p. 1399-1413.

182. Middelhoek, S., PERTURBATION WALLS IN THIN MAGNETIC DOUBLE PERMALLOY (Ni-Fe) FILMS. Applied Physics Letters, 1964. 5(4): p. 70-72.

183. Feldtkeller, E., Coupled Walls in Multilayer Films. Journal of Applied Physics, 1968. 39(2): p. 1181-1190.

184. Schafer, R., A. Hubert, and S.S.P. Parkin, Domain and domain wall observations in sputtered exchange-biased wedges. Magnetics, IEEE Transactions on, 1993. 29(6): p. 2738-2740.

185. McCord, J., et al., Magnetization processes in exchange biased giant magnetoresistive multilayer elements. Magnetics, IEEE Transactions on, 1996. 32(5): p. 4803-4805.

186. Portier, X. and A.K. Petford-Long, The formation of $360^{\circ}$ domain walls in magnetic tunnel junction elements. Applied Physics Letters, 2000. 76(6): p. 754756.

187. Muratov, C.B. and V.V. Osipov, Theory of $360^{\circ}$ domain walls in thin ferromagnetic films. Journal of Applied Physics, 2008. 104(5): p. 053908.

188. Diegel, M., R. Mattheis, and E. Halder, $360^{\circ}$ domain wall investigation for sensor applications. Magnetics, IEEE Transactions on, 2004. 40(4): p. 2655-2657.

189. Castaño, F.J., et al., Metastable states in magnetic nanorings. Physical Review B, 2003. 67(18): p. 184425.

190. Muratov, C.B. and V.V. Osipov, Bit Storage by $360^{\circ}$ Domain Walls in Ferromagnetic Nanorings. Magnetics, IEEE Transactions on, 2009. 45(8): p. 3207-3209.

191. Mascaro, M.D., C. Nam, and C.A. Ross, Interactions between $180^{\circ}$ and $360^{\circ}$ domain walls in magnetic multilayer stripes. Applied Physics Letters, 2010. 96(16): p. 162501. 
192. Marrows, C.H., Spin-polarised currents and magnetic domain walls. Advances in Physics, 2005. 54(8): p. 585-713.

193. Petit, D., et al., Domain wall pinning and potential landscapes created by constrictions and protrusions in ferromagnetic nanowires. Journal of Applied Physics, 2008. 103(11).

194. Lewis, E.R., et al., Magnetic domain wall pinning by a curved conduit. Applied Physics Letters, 2009. 95(15).

195. Yamaguchi, A., et al., Effect of Joule heating in current-driven domain wall motion. Applied Physics Letters, 2005. 86(1): p. 012511.

196. Thiaville, A., et al., Micromagnetic understanding of current-driven domain wall motion in patterned nanowires. Europhysics Letters (EPL), 2005. 69(6): p. 990996.

197. Ryu, J. and H.-W. Lee, Current-induced domain wall motion: Domain wall velocity fluctuations. Journal of Applied Physics, 2009. 105(9): p. 093929.

198. Martinez, E., et al., Thermal effects in domain wall motion: Micromagnetic simulations and analytical model. Physical Review B, 2007. 75(17).

199. Komine, T., et al., Reduction of intrinsic critical current density for currentinduced domain wall motion by using a ferrimagnetic nanowire with perpendicular magnetic anisotropy. Journal of Applied Physics, 2011. 109(7): p. $07 \mathrm{D} 503$.

200. Mascaro, M.D. and C.A. Ross, ac and dc current-induced motion of a $360^{\circ}$ domain wall. Physical Review B, 2010. 82(21): p. 214411.

201. Noh, S.J., et al., Effects of notch shape on the magnetic domain wall motion in nanowires with in-plane or perpendicular magnetic anisotropy. Journal of Applied Physics, 2012. 111(7): p. 07D123.

202. Kunz, A. and S.C. Reiff, Dependence of domain wall structure for low field injection into magnetic nanowires. Applied Physics Letters, 2009. 94(19): p. 192504.

203. Jang, Y., et al., Formation and structure of 360 and 540 degree domain walls in thin magnetic stripes. Applied Physics Letters, 2012. 100(6): p. 062407.

204. Geng, L.D. and Y.M. Jin, Generation and storage of $360^{\circ}$ domain walls in planar magnetic nanowires. Journal of Applied Physics, 2012. 112(8): p. 083903.

205. Jin, Y.M., Phase field modeling of current density distribution and effective electrical conductivity in complex microstructures. Applied Physics Letters, 2013. 103(2): p. 021906.

206. Zeng, H.T., et al., The influence of wire width on the charge distribution of transverse domain walls and their stray field interactions. Journal of Magnetism and Magnetic Materials, 2010. 322(14): p. 2010-2014.

207. Faulkner, C.C., Artificial domain wall nanotraps in $\mathrm{Ni}_{81} \mathrm{Fe}_{19}$ wires. Journal of Applied Physics, 2004. 95(11): p. 6717.

208. Petit, D., et al., Magnetic imaging of the pinning mechanism of asymmetric transverse domain walls in ferromagnetic nanowires. Applied Physics Letters, 2010. 97(23): p. 233102. 
209. Zeng, H.T., et al., Combined electrical and magneto-optical measurements of the magnetization reversal process at a domain wall trap. Applied Physics Letters, 2009. 94(10).

210. Petit, D., et al., High efficiency domain wall gate in ferromagnetic nanowires. Applied Physics Letters, 2008. 93(16).

211. Lewis, E.R., et al., Measuring Domain Wall Fidelity Lengths Using a Chirality Filter. Physical Review Letters, 2009. 102(5).

212. Moore, T.A., et al., Domain wall velocity measurement in permalloy nanowires with X-ray magnetic circular dichroism imaging and single shot Kerr microscopy. Journal of Magnetism and Magnetic Materials, 2010. 322(9-12): p. 1347-1352.

213. Moore, T.A., et al., Magnetic-field-induced domain-wall motion in permalloy nanowires with modified Gilbert damping. Physical Review B, 2010. 82(9): p. 094445.

214. Kläui, M., et al., Current-induced vortex nucleation and annihilation in vortex domain walls. Applied Physics Letters, 2006. 88(23): p. 232507.

215. Fernandez-Pacheco, A., et al., Domain wall conduit behavior in cobalt nanowires grown by focused electron beam induced deposition. Applied Physics Letters, 2009. 94(19).

216. Yamaguchi, A., et al., Temperature estimation in a ferromagnetic Fe-Ni nanowire involving a current-driven domain wall motion. Journal of Physics: Condensed Matter, 2012. 24(2): p. 024201.

217. Allwood, D.A., G. Xiong, and R.P. Cowburn, Writing and erasing data in magnetic domain wall logic systems. Journal of Applied Physics, 2006. 100(12).

218. Jamali, M., K.-J. Lee, and H. Yang, Effect of nonadiabatic spin transfer torque on domain wall resonance frequency and mass. Applied Physics Letters, 2011. 98 (9): p. 092501.

219. Annunziata, A.J., et al. Racetrack memory cell array with integrated magnetic tunnel junction readout. in Electron Devices Meeting (IEDM), 2011 IEEE International. 2011.

220. Weisheng, Z., et al. Racetrack memory based reconfigurable computing. in Faible Tension Faible Consommation (FTFC), 2013 IEEE. 2013.

221. Zhao, W.S., et al. Magnetic domain-wall racetrack memory for high density and fast data storage. in Solid-State and Integrated Circuit Technology (ICSICT), 2012 IEEE 11th International Conference on. 2012.

222. Shibata, J., et al., Current-induced magnetic vortex motion by spin-transfer torque. Physical Review B, 2006. 73(2): p. 020403.

223. Lee, K.-S., et al., Universal Criterion and Phase Diagram for Switching a Magnetic Vortex Core in Soft Magnetic Nanodots. Physical Review Letters, 2008. 101(26): p. 267206.

224. Kammerer, M., et al., Magnetic vortex core reversal by excitation of spin waves. Nat Commun, 2011. 2: p. 279.

225. Schafer, A.H.a.R., Magnetic Domains: The Analysis of Magnetic Microstructures, 2000.

226. Mascaro, M.D., Interactions between $180^{\circ}$ and $360^{\circ}$ domain walls in magnetic multilayer stripes. Appl. Phys. Lett., 2010. 96(16): p. 162501. 
227. Gambardella, P., et al., Ferromagnetism in one-dimensional monatomic metal chains. Nature, 2002. 416(6878): p. 301-304.

228. Gambardella, P., et al., Giant Magnetic Anisotropy of Single Cobalt Atoms and Nanoparticles. Science, 2003. 300(5622): p. 1130-1133.

229. Khajetoorians, A.A., et al., Realizing All-Spin-Based Logic Operations Atom by Atom. Science, 2011. 332(6033): p. 1062-1064.

230. Menzel, M., et al., Information Transfer by Vector Spin Chirality in Finite Magnetic Chains. Physical Review Letters, 2012. 108(19): p. 197204.

231. Wei, D., et al., Pd Atomic Chain Formation as a Result of Submonolayer Deposition of 3d Metals on Pd(110). Physical Review Letters, 2009. 103(22): p. 225504.

232. Mermin, N. and H. Wagner, Absence of Ferromagnetism or Antiferromagnetism in One- or Two-Dimensional Isotropic Heisenberg Models. Physical Review Letters, 1966. 17(22): p. 1133-1136.

233. Spišák, D. and J. Hafner, Magnetism of ultrathin wires suspended in free space and adsorbed on vicinal surfaces. Physical Review B, 2003. 67(21): p. 214416.

234. Mahdi, S. and S.S. Ataee, First principles study on spin and orbital magnetism of $3 d$ transition metal monatomic nanowires (Mn, Fe and Co). Journal of Physics: Condensed Matter, 2011. 23(12): p. 125301.

235. Tung, J.C. and G.Y. Guo, Ab initio studies of spin-spiral waves and exchange interactions in 3d transition metal atomic chains. Physical Review B, 2011. 83(14): p. 144403.

236. Töws, W. and G.M. Pastor, Theoretical study of the temperature dependence of the magnon dispersion relation in transition-metal wires and monolayers. Physical Review B, 2012. 86(5): p. 054443.

237. Mokrousov, Y., et al., Giant Magnetocrystalline Anisotropies of 4d TransitionMetal Monowires. Physical Review Letters, 2006. 96(14): p. 147201.

238. Tung, J.C. and Y.K. Wang, The structural, electronic, and magnetic properties of $\mathrm{Fe}-\mathrm{Ir}, \mathrm{Co}-\mathrm{Ir}$ and $\mathrm{Ni}-\mathrm{Ir}$ linear and zigzag nanowires: First-principles calculations. Journal of Magnetism and Magnetic Materials, 2011. 323(15): p. 2032-2036.

239. Ataca, C., et al., Structural, electronic, and magnetic properties of $3 d$ transition metal monatomic chains: First-principles calculations. Physical Review B, 2008. 77(21): p. 214413.

240. Nautiyal, T., T.H. Rho, and K.S. Kim, Nanowires for spintronics: A study of transition-metal elements of groups 8-10. Physical Review B, 2004. 69(19): p. 193404.

241. Hashemi, H., W. Hergert, and V.S. Stepanyuk, Magnetic states of M-Fe wires $(M=S c-N i)$ on vicinal $\mathrm{Cu}(111)$ from first principles. Physical Review B, 2010. 81(10): p. 104418.

242. Tung, J.C. and G.Y. Guo, An ab initio study of the magnetic and electronic properties of $\mathrm{Fe}, \mathrm{Co}$, and $\mathrm{Ni}$ nanowires on $\mathrm{Cu}(001)$ surface. Computer Physics Communications, 2011. 182(1): p. 84-86. 
243. Tung, J.C., Y.K. Wang, and G.Y. Guo, Magnetic anisotropy and spin-spiral wave in $\mathrm{V}, \mathrm{Cr}$ and $\mathrm{Mn}$ atomic chains on $\mathrm{Cu}\left(\begin{array}{l}0 \\ 0\end{array}\right)$ surface: first principles calculations. Journal of Physics D: Applied Physics, 2011. 44(20): p. 205003.

244. Hashemi, H., W. Hergert, and V.S. Stepanyuk, Magnetic properties of $3 d$ transition metal chains on vicinal surface. Journal of Magnetism and Magnetic Materials, 2010. 322(9-12): p. 1296-1299.

245. Luo, S.J., G.Y. Guo, and A. Laref, Magnetism of 3d-Transition Metal (Fe, Co, and Ni) Nanowires on w-BN (0001). The Journal of Physical Chemistry C, 2009. 113(33): p. 14615-14622.

246. Dallmeyer, A., et al., Electronic states and magnetism of monatomic $\mathrm{Co}$ and $\mathrm{Cu}$ wires. Physical Review B, 2000. 61(8): p. R5133-R5136.

247. Rodrigues, V., et al., Evidence for Spontaneous Spin-Polarized Transport in Magnetic Nanowires. Physical Review Letters, 2003. 91(9): p. 096801.

248. Antropov, V.P., et al., Spin dynamics in magnets: Equation of motion and finite temperature effects. Physical Review B, 1996. 54(2): p. 1019-1035.

249. Böttcher, D., A. Ernst, and J. Henk, Atomistic magnetization dynamics in nanostructures based on first principles calculations: application to Co nanoislands on $\mathrm{Cu}(111)$. Journal of Physics: Condensed Matter, 2011. 23(29): p. 296003.

250. Skubic, B., et al., A method for atomistic spin dynamics simulations: implementation and examples. Journal of Physics: Condensed Matter, 2008. 20(31): p. 315203.

251. Magiera, M.P., et al., Spin waves cause non-linear friction. EPL (Europhysics Letters), 2011. 95(1): p. 17010.

252. Kazantseva, N., et al., Towards multiscale modeling of magnetic materials: Simulations of FePt. Physical Review B, 2008. 77(18): p. 184428.

253. David, S.G.B., et al., Thermally activated magnetization reversal in monatomic magnetic chains on surfaces studied by classical atomistic spin-dynamics simulations. Journal of Physics: Condensed Matter, 2011. 23(39): p. 394204.

254. Kresse, G. and J. Furthmüller, Efficient iterative schemes for ab initio totalenergy calculations using a plane-wave basis set. Physical Review B, 1996. 54(16): p. 11169-11186.

255. Kresse, G. and J. Hafner, Ab initio molecular dynamics for liquid metals. Physical Review B, 1993. 47(1): p. 558-561.

256. Kresse, G. and J. Hafner, Ab initio molecular-dynamics simulation of the liquidmetal-amorphous-semiconductor transition in germanium. Physical Review B, 1994. 49(20): p. 14251-14269.

257. Blöchl, P.E., Projector augmented-wave method. Physical Review B, 1994. 50(24): p. 17953-17979.

258. Kresse, G. and D. Joubert, From ultrasoft pseudopotentials to the projector augmented-wave method. Physical Review B, 1999. 59(3): p. 1758-1775.

259. Perdew, J.P., K. Burke, and M. Ernzerhof, Generalized Gradient Approximation Made Simple. Physical Review Letters, 1996. 77(18): p. 3865-3868. 
260. Perdew, J.P., K. Burke, and M. Ernzerhof, Generalized Gradient Approximation Made Simple [Phys. Rev. Lett. 77, 3865 (1996)]. Physical Review Letters, 1997. 78(7): p. 1396-1396.

261. Shallcross, S., et al., An ab initio effective Hamiltonian for magnetism including longitudinal spin fluctuations. Physical Review B, 2005. 72(10): p. 104437.

262. Kittel, C., Model of Exchange-Inversion Magnetization. Physical Review, 1960. 120(2): p. 335-342.

263. Harris, E.A. and J. Owen, Biquadratic Exchange Between $\mathrm{Mn}^{2+}$ Ions in $\mathrm{MgO}$. Physical Review Letters, 1963. 11(1): p. 9-10.

264. Rosengaard, N.M. and B. Johansson, Finite-temperature study of itinerant ferromagnetism in Fe, Co, and Ni. Physical Review B, 1997. 55(22): p. 1497514986.

265. Iwashita, T. and N. Uryu, The Curie temperature of the Ising ferromagnet with higher-order exchange interaction. Journal of Physics C: Solid State Physics, 1984. 17(5): p. 855.

266. Mryasov, O.N., et al., Temperature-dependent magnetic properties of FePt: Effective spin Hamiltonian model. EPL (Europhysics Letters), 2005. 69(5): p. 805.

267. Wolverton, C. and A. Zunger, First-principles theory of short-range order, electronic excitations, and spin polarization in $\mathrm{Ni}-\mathrm{V}$ and $\mathrm{Pd}-\mathrm{V}$ alloys. Physical Review B, 1995. 52(12): p. 8813-8828.

268. Vindigni, A., et al., Fast switching of bistable magnetic nanowires through collective spin reversal. Applied Physics Letters, 2005. 87(7): p. - .

269. Deeley, E.M. and B. Saha, Neel wall energy in thin films for unequal magnetization angles. Magnetics, IEEE Transactions on, 1988. 24(6): p. 23832385. 


\section{Appendix A: Domain Wall with Substructures in Ideal}

\section{Magnetic Film}

The complete analytical calculations for domain wall with substructures are difficult, so that some approximations should be made. At first, in a reasonable approximation, $\theta$ mainly depends on $y$ while $\varphi$ mainly depends on $x$, as schematically shown by Fig. A1. Besides, magnetic charges will be generated due to the formation of substructures of Neel type, which will result in higher magnetostatic energy. Such additional magnetiostatic energy makes the analytical calculation complicated, but in a nonrigid approximation, the energy can be treated as anisotropy energy of some kind, noted by $K_{y}$ which is applied only to substructures, just like the shape anisotropy. The value of $K_{y}$ can be approximated by[269]

$$
K_{y}=\frac{\pi}{8}\left(\frac{t}{t+w}\right) \mu_{0} M_{s}^{2}
$$

where $t$ is the film thickness and $w$ is the DW width which can be given by $w=2 \lambda_{D W}$ with $\lambda_{D W}$ the DW parameter. As for those material parameters used in this paper, it can be approximated that $K_{y}=0.079 \mu_{0} M_{s}^{2}$.Thus, the total energy including exchange energy, magnetocrystalline anisotropy energy, and magnetostatic energy will be

$$
e_{t o t}=A\left[\left(\frac{\partial \theta}{\partial y}\right)^{2}+\sin ^{2} \theta\left(\frac{\partial \varphi}{\partial x}\right)^{2}\right]+\sin ^{2} \theta\left(K+K_{y} \sin ^{2} \varphi\right)
$$


where $K=K_{u}-K_{d}$ with $K_{u}$ the magnetocrystalline anisotropy constant and $K_{d}=0.5 \mu_{0} M_{s}^{2}$ the demagnetization energy of thin film.

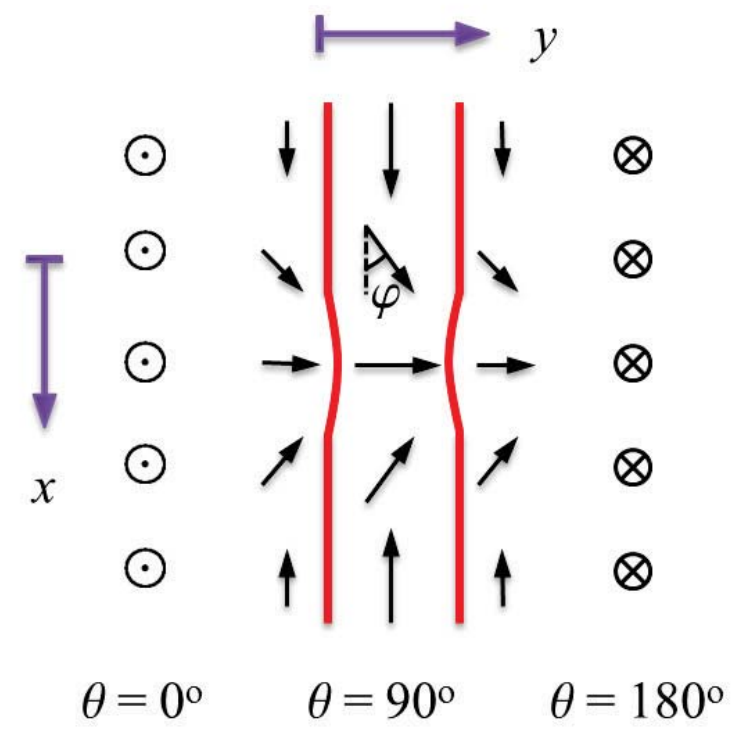

Figure A1 Schematic diagram for the magnetization structure of a substructure within a DW.

The DW substructure can be obtained by

$$
\left\{\begin{array}{l}
\frac{\delta e_{t o t}}{\delta \theta}=-2 A \frac{\partial^{2} \theta}{\partial y^{2}}+2 \sin \theta \cos \theta\left[K+K_{y} \sin ^{2} \varphi+A\left(\frac{\partial \varphi}{\partial x}\right)^{2}\right]=0 \\
\frac{\delta e_{t o t}}{\delta \varphi}=2 \sin ^{2} \theta\left[K_{y} \sin \varphi \cos \varphi-A \frac{\partial^{2} \varphi}{\partial x^{2}}\right]=0
\end{array}\right.
$$

from which one can obtain the substructure width parameters $\lambda_{X}$ and $\lambda_{Y}$, along $\mathrm{X}$ and $\mathrm{Y}$, respectively, as well as DW width parameter $\lambda_{D W}$,

$$
\left\{\begin{array}{l}
\lambda_{X}=\sqrt{A / K_{y}} \\
\lambda_{Y}=\sqrt{A /\left(K+2 K_{y} \sin ^{2} \varphi\right)} \\
\lambda_{D W}=\sqrt{A / K}
\end{array}\right.
$$


The total energy for substructurewith length $w_{X}=2 \lambda_{X}$ is given by

$$
\begin{aligned}
E & =\iint e_{t o t} d x d y=4 \sqrt{A K} \int_{-\lambda_{X}}^{\lambda_{X}} \sqrt{1+\frac{2 K_{y}}{K} \operatorname{sech}^{2} \frac{x}{\lambda_{X}}} d x \\
& =4 \sqrt{A K} \times 2 \lambda_{X} \int_{0}^{1} \sqrt{1+\frac{2 K_{y}}{K} \operatorname{sech}^{2} \tilde{x}} d \tilde{x} \\
& =2 \eta \lambda_{X} \gamma_{D W}
\end{aligned}
$$

where $\eta=\int_{0}^{1} \sqrt{1+\frac{2 K_{y}}{K} \operatorname{sech}^{2} \tilde{x}} d \ddot{x}>1$ is a coefficient multiplied on the DW energy density $\gamma_{D W}=4 \sqrt{A K}$. It is approximated that $\eta \approx 1.20$ with substructure length $w_{X} \approx 22.5 \mathrm{~nm}$.

When a magnetic field $H$ along Z-direction is applied, there will be an additional energy term in Eq. (A2), $e_{H}=-H M_{s} \cos \theta$. The LLG equation will be

$$
\left\{\begin{array}{l}
\dot{\theta}=-\frac{2 \gamma \sin \theta}{M_{s}}\left[-A \frac{\partial^{2} \varphi}{\partial x^{2}}+K_{y} \sin \varphi \cos \varphi\right]-\alpha \sin \theta \dot{\varphi} \\
\sin \theta \dot{\varphi}=\frac{\gamma}{M_{s}}\left[-2 A \frac{\partial^{2} \theta}{\partial y^{2}}+2 \sin \theta \cos \theta\left(K+K_{y} \sin ^{2} \varphi+A\left(\frac{\partial \varphi}{\partial x}\right)^{2}\right)+H M_{s} \sin \theta\right]+\alpha \hat{\theta}
\end{array}\right.
$$

Using the same assumption in Sec. 3.3, we can calculate the substructure velocities in $\mathrm{X}$ and $\mathrm{Y}$-direction,

$$
\left\{\begin{array}{l}
v_{X}=-\frac{\gamma L H}{\left(1+\alpha^{2}\right) \pi N} \\
v_{Y}=\frac{\alpha \gamma \lambda_{Y} H}{1+\alpha^{2}}
\end{array}\right.
$$

where $L$ is the DW length and $N$ is the number of substructures. It is noted that both $v_{X}$ and $v_{Y}$ are linearly related to magnetic field $H$, while for $v_{X}$, it is also inversely 
proportional to the substructure density $\omega=N / L$ and moves along $-\mathrm{X}$ direction for the ccw-hh substructure shown in Fig. A1.

For DW without substructures, with applied field $H$, the DW velocity is given by

$$
v=\frac{\gamma \lambda H}{\alpha}=\frac{\gamma \lambda K_{y} \sin 2 \varphi}{M_{s}},
$$

with $\lambda=\sqrt{A /\left(K+K_{y} \sin ^{2} \varphi\right)}$. 


\section{Appendix B: Domain Wall with Substructures in}

\section{Defective Magnetic Film}

In real magnetic films, since imperfections exist, pinning sites should be taken into account for DW motion, and pinning barriers $V_{\text {pin }}(\theta, \varphi, x, y)$ should be added to the total energy. Then, we have

$$
\left\{\begin{array}{l}
\dot{\theta}=-\frac{\gamma \sin \theta}{M_{s}}\left[-2 A \frac{\partial^{2} \varphi}{\partial x^{2}}+2 K_{y} \sin \varphi \cos \varphi-f_{2}\right]-\alpha \sin \theta \dot{\varphi} \\
\sin \theta \dot{\varphi}=\frac{\gamma}{M_{s}}\left[-2 A \frac{\partial^{2} \theta}{\partial y^{2}}+2 \sin \theta \cos \theta\left(K+K_{y} \sin ^{2} \varphi+A\left(\frac{\partial \varphi}{\partial x}\right)^{2}\right)+H M_{s} \sin \theta-f_{1}\right]+\alpha \dot{\theta}
\end{array},\right.
$$

where the pinning forces are $f_{1}=-\delta V_{\text {pin }} / \delta \theta$ and $f_{2}=-\delta V_{\text {pin }} / \delta \varphi$. Since the exact form of pinning forces is unknown, in an approximation, at wall center, i.e., $\theta \rightarrow \pi / 2$, we have the substructure velocity along Y-direction by ignoring the drag effect of DW segments,

$$
v_{Y}=\frac{\alpha \gamma \lambda_{Y}}{1+\alpha^{2}}\left(H-\frac{f_{1}+f_{2} / \alpha}{M_{s}}\right),
$$

which can be compared with the DW velocity,

$$
v=\frac{\gamma \lambda}{\alpha}\left(H-\frac{f_{1}}{M_{s}}\right)
$$

It is noted that substructures will overcome larger pinning force than DWs due to the additional motion freedom degree in X-direction. It is also noted that the mobility for substructure $m_{Y}$ is much lower than that for DW $m$, and their ratio is 


$$
\frac{m_{Y}}{m} \approx \frac{\alpha^{2}}{1+\alpha^{2}} .
$$

For finite temperature, the thermal effect is effectively introduced by the Gaussian distributed fluctuation field $\mathbf{H}_{t h}$ with certain amplitude, and hence the corresponding energy term will be

$$
e_{t h}=-H_{t h, z} M_{s} \cos \theta-H_{t h, y} M_{s} \sin \theta \sin \varphi-H_{t h, x} M_{s} \sin \theta \cos \varphi
$$

In an approximation, at wall center, without considering the pinning effect or external magnetic field, we have the substructure velocity along Y-direction by ignoring the drag effect of DW segments,

$$
v_{Y}=\frac{\alpha \gamma \lambda_{Y}}{1+\alpha^{2}}\left(H_{t h, z}+\frac{H_{t h, x}}{\alpha}\right)
$$

which can be compared with the DW velocity,

$$
v=\frac{\gamma \lambda}{\alpha} H_{t h, z} .
$$

It is noted that the thermal field will have an enhanced effect on substructures, and the averaged amplitude is

$$
\sigma_{Y}^{2}=\frac{1+\alpha^{2}}{\alpha^{2}} \sigma^{2}
$$

much larger than the thermal effect on DW. 


\section{Appendix C: Copyright Permission of Section 4.2 and}

\section{Section 6.2}

Section 4.2 of Chapter 4 and Section 6.2 of Chapter 6 in this dissertation were partially originally published in "Generation and storage of $360^{\circ}$ domain walls in planar magnetic nanowires", Journal of Applied Physics, AIP Publishing LLC, 2012, 112, page 083903. Permission to reuse materials from the published work was obtained with kind permission from AIP Publishing LLC. A copy of the granting permission for reproduction is shown as below.

$\begin{array}{ll}\text { License Number } & \text { 3681530099788 } \\ \text { Order Date } & \text { Aug 03, 2015 } \\ \text { Publisher } & \text { AlP Publishing LLC } \\ \text { Publication } & \text { Journal of Applied Physics } \\ \text { Article Title } & \text { Generation and storage of 360 domain } \\ & \text { walls in planar magnetic nanowires } \\ \text { Author } & \text { Liwei D. Geng, Yongmei M. Jin } \\ \text { Online Publication Date } & \text { Oct 17, 2012 } \\ \text { Volume number } & 112 \\ \text { Issue number } & 8 \\ \text { Type of Use } & \text { Thesis/Dissertation } \\ \text { Requestor type } & \text { Author (original article) } \\ \text { Format } & \text { Print and electronic } \\ \text { Portion } & \text { Excerpt (> 800 words) } \\ \text { Will you be translating? } & \text { No }\end{array}$


Title of your thesis /

dissertation

Expected completion date

Estimated size (number of

pages)

Total
STRUCTURES, PROPERTIES AND

FUNCTIONALITIES OF MAGNETIC

DOMAIN WALLS IN THIN FILMS,

NANOWIRES AND ATOMIC CHAINS:

MICROMAGNETIC AND AB INITIO

STUDIES

Aug 2015

270

0.00 USD 


\section{Appendix D: Copyright Permission of Section 4.3 and}

\section{Section 6.2}

Section 4.3 of Chapter 4 and part of Section 6.2 of Chapter 6 in this dissertation were originally published in "Altering critical depinning current via domain wall pile-up in magnetic nanowires", Journal of Magnetism and Magnetic Materials, Elsevier, 2015, 393, page 121-126. Permission to reuse materials from the published work was obtained with kind permission from Elsevier. A copy of the granting permission for reproduction is shown as below.

This is a License Agreement between Liwei Geng ("You") and Elsevier ("Elsevier") provided by Copyright Clearance Center ("CCC"). The license consists of your order details, the terms and conditions provided by Elsevier, and the payment terms and conditions.

$\begin{array}{ll}\text { Supplier } & \begin{array}{l}\text { Elsevier Limited } \\ \text { The Boulevard, Langford Lane } \\ \text { Kidlington, Oxford, OX5 1GB, UK }\end{array} \\ \text { Registered Company } & \\ \text { Number } & 1982084 \\ \text { Customer name } & \text { Liwei Geng } \\ \text { Customer address } & 317 \text { Shelden Ave Apt } 5 \\ & \text { HOUGHTON, MI 49931 } \\ \text { License number } & \text { Aug 03, 2015 } \\ \text { License date } & \text { Elsevier } \\ \text { Licensed content publisher } & \text { Journal of Magnetism and Magnetic } \\ \text { Licensed content publication } & \text { Materials } \\ \text { Licensed content title } & \begin{array}{l}\text { Altering critical depinning current via } \\ \text { domain wall pile- up in magnetic }\end{array} \\ & \text { nanowires }\end{array}$


Licensed content author

Licensed content date

Licensed content volume

Number

Licensed content issue

Number

Number of pages

Start Page

End Page

Type of Use

Intended publisher of new

Work

Portion

Format

Are you the author of this

Elsevier article?

Will you be translating?

Title of your

thesis/dissertation
Liwei D. Geng, Yongmei M. J in

1 November 2015

393

$\mathrm{n} / \mathrm{a}$

6

121

126

reuse in a thesis/dissertation

other

full article

both print and electronic

Yes

No

STRUCTURES, PROPERTIES AND

FUNCTIONALITIES OF MAGNETIC

DOMAIN WALLS IN THIN FILMS, NANOWIRES AND ATOMIC CHAINS:

MICROMAGNETIC AND AB INITIO

STUDIES

Expected completion date

Estimated size (number of

pages)

Elsevier VAT number

Permissions price

VAT/Local Sales Tax

Total
Aug 2015

270

GB 494627212

0.00 USD

0.00 USD / 0.00 GBP

0.00 USD 